University of Louisville

ThinkIR: The University of Louisville's Institutional Repository

Electronic Theses and Dissertations

$5-2021$

\title{
The neural architecture of emotional intelligence.
}

Teodora Stoica

University of Louisville

Follow this and additional works at: https://ir.library.louisville.edu/etd

Part of the Cognitive Neuroscience Commons

\section{Recommended Citation}

Stoica, Teodora, "The neural architecture of emotional intelligence." (2021). Electronic Theses and Dissertations. Paper 3623.

https://doi.org/10.18297/etd/3623

This Doctoral Dissertation is brought to you for free and open access by ThinkIR: The University of Louisville's Institutional Repository. It has been accepted for inclusion in Electronic Theses and Dissertations by an authorized administrator of ThinkIR: The University of Louisville's Institutional Repository. This title appears here courtesy of the author, who has retained all other copyrights. For more information, please contact thinkir@louisville.edu. 


\title{
THE NEURAL ARCHITECTURE OF EMOTIONAL INTELLIGENCE
}

\author{
By
}

\section{Teodora Stoica}

B.A., East Carolina University, 2007

M.S., University of Hartford, 2010

\author{
A Dissertation \\ Submitted to the Faculty of the \\ Graduate School at the University of Louisville \\ In Partial Fulfillment of the Requirements for the degree of \\ Doctor of Philosophy \\ In Interdisciplinary Studies: Translational Neuroscience \\ Department of Interdisciplinary Studies \\ University of Louisville \\ Louisville, Kentucky
}

May 2021 



\section{THE NEURAL ARCHITECTURE OF EMOTIONAL INTELLIGENCE}

By

Teodora Stoica

B.A., East Carolina University, 2007

M.S., University of Hartford, 2010

A Dissertation Approved on

April 9, 2021

by the following Dissertation Committee:

Brendan Depue

Dissertation Mentor \& Committee Chair

Nick Hindy

Tamara Newton

Rif S. El-Mallakh

David Magnuson

Cynthia Corbitt 


\section{DEDICATION}

To the human brain,

"what may be the most complex object in existence, one wet kilogram within which spin universes."

- Anthony Doerr 


\section{ACKNOWLEDGEMENTS}

To my advisor: who, on my first day of graduate school told me not to be scared, but to be aware.

To my committee: Dr. Nick Hindy, Dr. Tamara Newton, Dr. Rif S. El-Mallakh, Dr. David Magnuson and Dr. Cindy Corbitt for contributing their valuable time to reviewing this dissertation.

To every member of NILCAMP that has passed through and taught me that doing great science doesn't have to look like this:

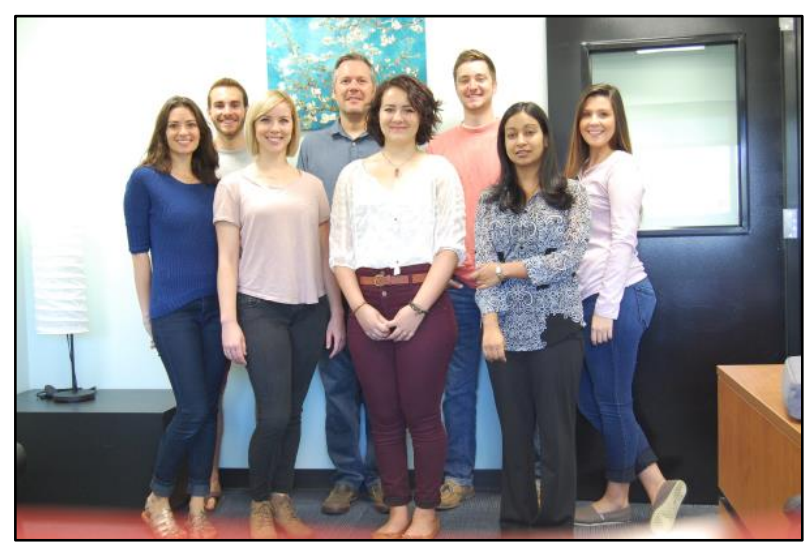

And can definitely look like this:

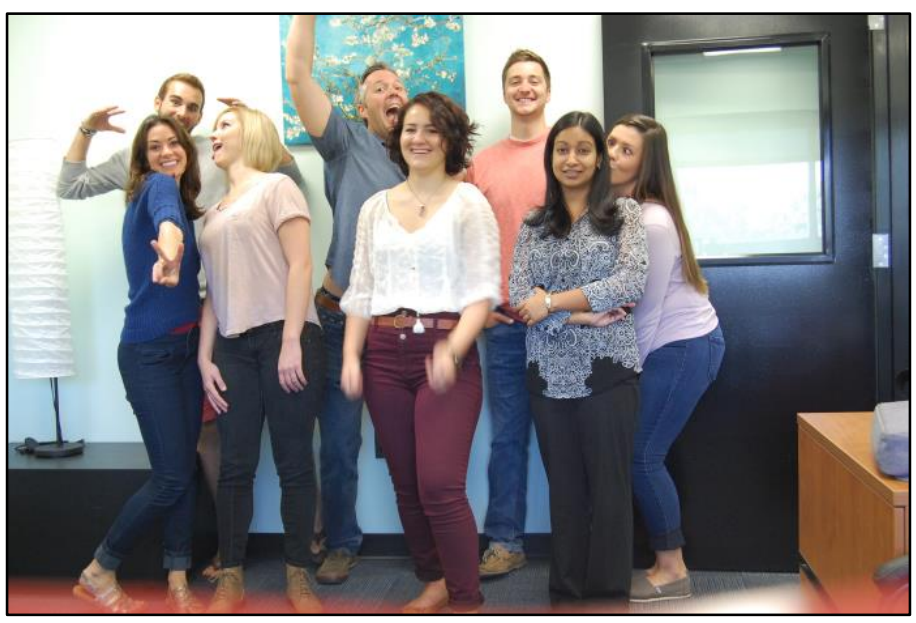


To my parents, who alleviated the agony of the $\mathrm{PhD}$ Program with a myriad of travel experiences.

To my mother, who reminded me to rise above the noise.

To my grandmother, who taught me to see the bigger picture.

To my best friend, Ashley Katz, whose unending encouragement shattered all my self-doubt.

To Lauren, who reminded me to stop crying and just eat some noods.

To Sean, whose sincere and generous spirit is without match.

To Bassil, who reminds me of what my inner child always needs.

To Cat, a shelter through the roughest storms.

To running, that taught me the PhD program isn't a sprint, but an Ultra-Marathon.

To my little sister Vali, who, for some reason, thinks it's cool I'm a nerd.

To Nelleke, who is a paramount example of a terrific human being.

To Joseph, the ideal mental sparring partner.

To lan, who radiates joy throughout the darkest of days.

To Cherokee, Iroquois and Jefferson Memorial Parks in Louisville for providing me with fresh air.

To Dr. Andrea Olinger, who taught me not to be afraid of the empty page.

To Brenda, who weaved herself into my life tapestry.

To my childhood friends Oana and Luciana, who remind me of my roots.

To Keith, who really believed my "unusual amount of stick-to-it-ness" will get me here.

To George, and his unending self-disclosing voicemails.

To Benton, for seeing my soul's shape and his family, for providing me with a sense of home.

To Anna, who despite my grumblings, taught me how to make pretty sounds.

To Stacy, who pulled me through the last year of my PhD by simply reminding me to "breathe through it".

To Kamryn, without whom I really couldn't see a way forward.

To both Amys, each healing me in their own ways.

To Sara, whose realness always grounds me.

To the Shelby Park Community Garden, for letting me grow.

For Surya, who always thought everything was "gonna be fine".

To all the young scientists I met through Louisville and Kentucky Science Pathways, that rekindled my passion for science.

To Mona. To Mona. To Mona.

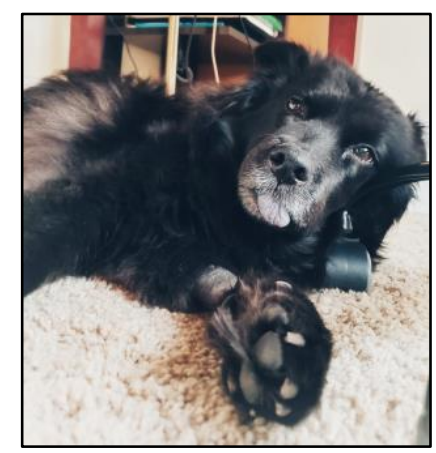




\begin{abstract}
THE NEURAL ARCHITECTURE OF EMOTIONAL INTELLIGENCE

Teodora Stoica
\end{abstract}

April 9, 2021

Emotional Intelligence (EI) is a nebulous concept that permeates daily interpersonal communication. Despite prolific research into its benefits, El subjective measurement is difficult, contributing to an enigmatic definition of its core constructs. However, neuroimaging research probing socioaffective brain mechanisms underlying putative El constructs can add an objective perspective to existing models, thereby illuminating the nature of El. Therefore, the primary aim of this dissertation is to identify brain networks underlying El and examine how El arises from the brain's functional and structural neuroarchitecture. El is first defined according to behavioral data, which suggests El is made up of two core constructs: Empathy and Emotion Regulation (ER). The interaction of brain networks underlying Empathy and ER is then investigated using a novel neuroimaging analysis method: dynamic functional connectivity (dynFC). The results suggest efficient communication and (re)configuration between the CEN, DMN, SN underlie both ER and RME task dynamics, and that these temporal patterns relate to trait empathy and ER tendency. Given the demonstrated behavioral and neurobiological relationship between empathy and $\mathrm{ER}$, our 
second aim is to examine each of these constructs individually through detailed experiments using a variety of neuroimaging methodologies. The dissertation concludes by proposing El is an ability that arises from the effective, yet flexible communication between brain networks underlying Empathy and ER.

The dissertation is divided into five chapters. Chapter I describes the foundational concept of El as originally described by a variety of psychological figures and the lacuna that exists in terms of its neural correlates. Chapter II presents behavioral data that proposes El is best predicted by Empathy and ER. Chapter III explores the dynamic relationship between brain networks underlying Empathy and ER, with the aim of elucidating their neurobiological associations, and investigate how such associations may combine to create El. Chapter IV examines Empathy closely, by probing its neurobiological relationship to interoception and anxiety. Chapter V examines ER closely, by investigating whether gender plays a role in ER, and its neurobiological relationship to hormones. Chapter VI links the general findings from Chapters III, IV and V, and proposes an integrative neurocognitive El model. The dissertation concludes by providing clinical and non-clinical applications for the model. 


\section{TABLE OF CONTENTS}

PAGE

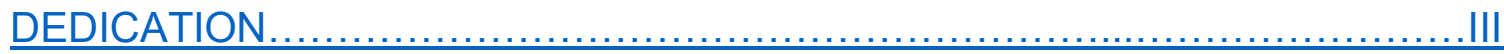

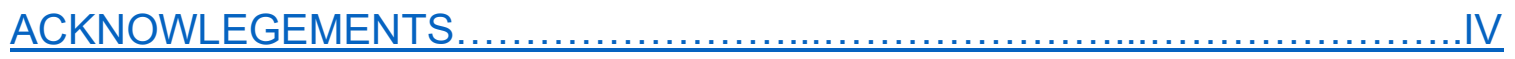

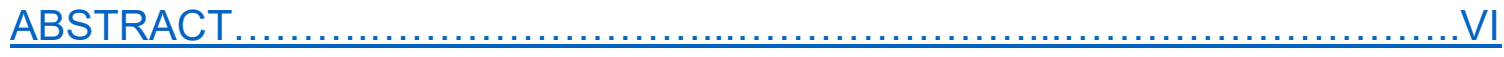

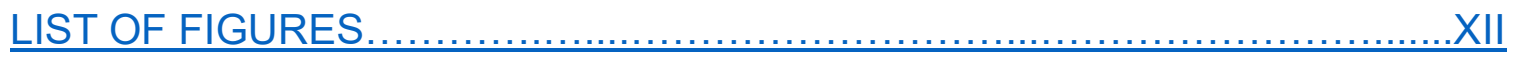

CHAPTER I: INTRODUCTION

Background ................................................ 10

Challenges of Current El Research ...............................13

Linking El to Neuroarchitecture .................................15

Neural Correlates of Emotional El Processes.....................17

Neural Correlates of Cognitive El Processes.....................20

Project Motivation .................................................23

CHAPTER II: BUILDING A COGNITIVE-EMOTIONAL EI FRAMEWORK

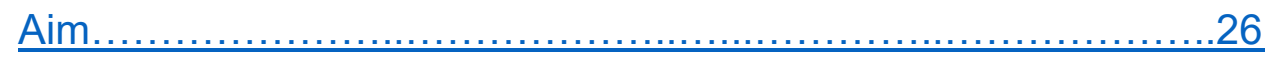

Hypotheses.....................................................27

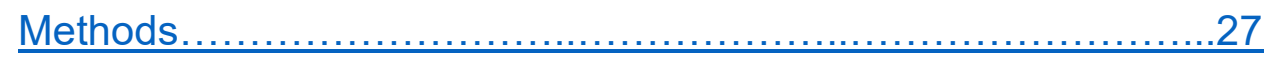

Results ...........................................................

CHAPTER III: EMPATHY AND EMOTION REGULATION

Experiment 1: Empathy and Emotion Regulation Brain Dynamics. 
Aim.

Hypotheses.

Methods. 40

Neuroimaging Results .51

Discussion. 55

\section{CHAPTER IV: A CLOSER LOOK AT EMPATHY}

Experiment 2: Shared Characteristics of Intrinsic Connectivity Networks Underlying Interoceptive Awareness and

Empathy. .63

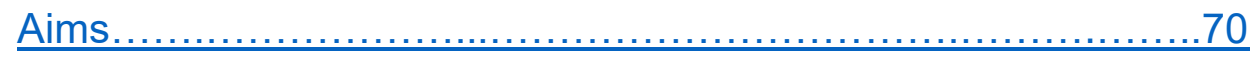

Hypotheses.......................................................... 71

Methods.......................................................... 71

Results.............................................................. 76

Discussion ...................................................... 82

Experiment 3: Convergent Neural Correlates of Empathy and

Anxiety During Socioemotional Processing....................92

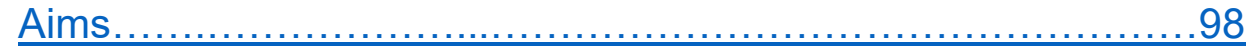

$\underline{\text { Hypotheses } \ldots \ldots \ldots \ldots \ldots \ldots \ldots \ldots \ldots \ldots \ldots \ldots \ldots \ldots \ldots \ldots \ldots \ldots \ldots \ldots \ldots \ldots \ldots \ldots \ldots}$

Methods ...........................................................99

Results....................................................... 107

Discussion ...................................................120

\section{CHAPTER V: A CLOSER LOOK AT EMOTION REGULATION}

Experiment 4: Cortical Morphometry and Structural Connectivity Relate to Executive Function and Estradiol Level in Healthy Adolescents...............................................133 
Aims.

Hypotheses....................................................139

Methods ..........................................................140

Results..................................................... 148

Discussion.......................................................156

Experiment 5: Gender Differences in Functional Connectivity during Emotion Regulation...................................164

Aim ......................................................168

Hypotheses..............................................168

Methods...........................................................169

Results............................................................179

Discussion............................................... 190

\section{CHAPTER VI: GENERAL DISCUSSION}

An Integrative Neurocognitive El Model........................198

Clinical Implications ............................................204

Future Directions ...........................................205

Conclusion .............................................206

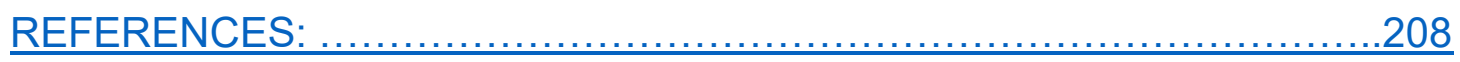

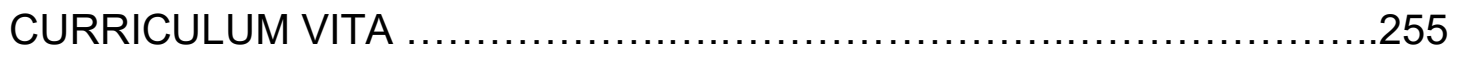




\section{LIST OF FIGURES}

\section{FIGURE \\ PAGE}

1. The Salience, Default Mode and Central Executive brain networks......16

2. El Emotional Processes................................................20

3. El Cognitive Processes.............................................22

4. El processes investigated for Cognitive-Emotional El Framework.......26

5. Behavioral El Correlational Network .................................33

6. Example of a Brain State.............................................. 39

7. Example trial from RME task .......................................42

8. Example ER Baseline Trial..........................................44

9. Example ER Trial.................................................. 45

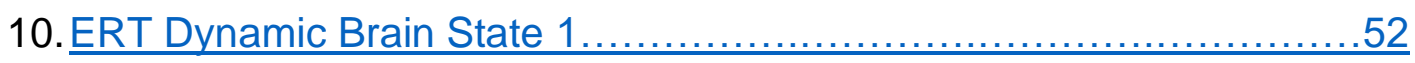

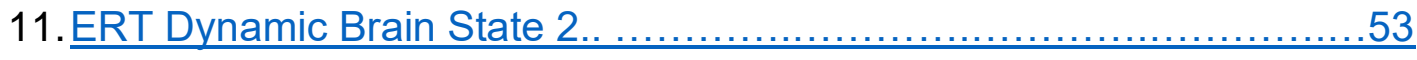

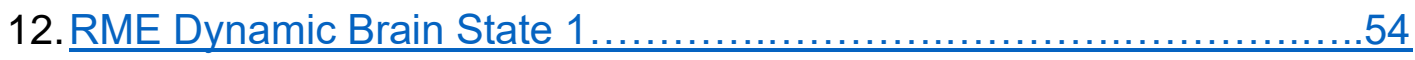

13. Experiment 1: Connectivity Analysis, Empathy and Interoception.........79

14. Experiment 1: Network Variability Analysis, Affective and MAIA..........80

15. Experiment 1: Network Variability Analysis, Cognitive and MAIA.........81

16. Experiment 2: Example Trial from Face Processing Task.............102

17. Experiment 2: Behavioral Results...................................109

18. Experiment 2: Neuroimaging Task Results............................111 
19. Experiment 2: Neuroimaging Results Regressed with Questionnaires.113

20. Experiment 2: Median Splits for Questionnaires, Behavior...............116

21. Experiment 2: Functional Connectivity Results......................118

22. Experiment 2: Median Splits for Questionnaires, Neuroimaging.........120

23. Experiment 2: Summary Figure of Neuroimaging Findings.............125

24. Experiment 3: Normalized Estradiol Values............................144

25. Experiment 3: EF behavior, cortical morphometry and connectivity.....152

26. Experiment 3: Estradiol relationship with structural connectivity.........154

27. Experiment 3: BRIEF subscales, estradiol and morphometry ...........155

28. Experiment 4: Single ER Baseline Trial............................170

29. Experiment 4: Single ER Trial....................................... 172

30. Experiment 4: Gender Differences in Negative Affect, ER Baseline...... 181

31. Experiment 4: Gender Differences in Negative Affect, ER Task.........182

32. Experiment 4: Gender Differences in Down-Regulation Success.........183

33. Experiment 4: Gender Differences in FC, ER Baseline..................184

34. Experiment 4: Gender Differences in FC, ER Task.......................186

35. Experiment 4: Gender Differences in FC, regressed with affect.........187

36. Experiment 4: Gender Differences in FC, regressed with suppression..189

37. Experiment 4: Proposed Neurocognitive El Model......................199 


\section{CHAPTER I: INTRODUCTION}

\section{"When we speak of emotional intelligence, we are alluding to whether someone understands key components of emotional functioning"}

Alain de Botton

\section{Background}

In recent years, an increased interest in the neurobiological basis of emotion perception and regulation in humans has led to the acknowledgement that emotions play a critical role in cognitive processes such as judgment, decision-making, problem solving and interpersonal perception (Damasio, 2005; Grewal et al., 2006). The novel concept of Emotional Intelligence (EI) proposes a useful framework for integrating aspects of emotion processing, emotion regulation and subsequent effective behavioral responses to emotional stimuli. This framework has its early roots in Thorndike's 1920 concept of social intelligence, which involved the ability to understand, manage, and interact wisely with others. It is also related to Gardner's 1986 discussion of intra- and interpersonal intelligence, which involved the capacity to understand the intentions, motivations and desires of the self and other, respectively. However, it was Mayer and Salovey that in 1990 built upon this work and formally defined El as: "a form of social intelligence that involves the ability to monitor one's own and 
others' feelings and emotions, to discriminate among them, and to use this information to guide one's thinking and action". This definition was later refined and broken down into four proposed abilities, or branches, that are distinct yet related: perceiving, using, understanding, and managing emotions (Mayer, 2004), and updated in 2016 by the same authors. The following is a description of the latest reformulation of their El model (Mayer et al., 2016).

The first branch, perceiving emotions, is the ability to detect and decipher emotions in own's own physical state, as well as others' faces, pictures and voices through their facial expressions, language and behavior. The perception of emotion is considered the most basic, as it provides the basis for all other emotional processing. The second branch, using emotions, is the ability to harness emotions and facilitate various cognitive activities, such as thinking and problem solving. For example, research suggests completing careful methodical tasks is best facilitated by a sad mood, while a good mood can stimulate creative and innovative thinking (Isen et al., 1991). An emotionally intelligent person could successfully gauge their mood and choose the task appropriately. The third branch, understanding emotions, is the ability to comprehend emotion language and appreciate complicated relationships among emotions. This ability encompasses the ability to correctly label emotions, be sensitive to variations in the same emotions (emotional granularity (Barrett, 2004)), and understand how a person might feel under certain conditions, or affective forecasting. The fourth branch, managing emotions, consists of the ability to regulate emotions in both 
ourselves and others. This branch includes the ability to evaluate strategies to maintain, reduce, or intensify an emotional response, and effectively manage one's own or others' emotions to achieve a desired outcome. For example, a shrewd politician may tap into feelings of anger in order to rouse feelings of anger in his political base. Other researchers have expanded on the Mayer \& Salovey El model, introducing the expression of emotions in a productive manner (Goleman, 1995), empathy (Leiberg \& Anders, 2006; Petrides \& Furnham, 2000), and even zeal to motivate others (Davies et al., 1998) as crucial social skills that can contribute to effectively managing relationships with others.

Despite its amorphous definition, skills linked to El have been directly associated with positive social interaction and well-being, while emotion dysregulation is considered a key mechanism underlying various psychopathologies (Davidson, 1998; M. L. Phillips et al., 2008). For example, research suggests high El measurements are inversely related to social anxiety and depression (Mayer et al., 1999), are associated with more positive interactions with other people (Lopes et al., 2005), less violent behavior (Brackett et al., 2004), improved relationship satisfaction, leadership ability, career success, physical/emotional health, and many others (Brackett et al., 2012; Hagelskamp et al., 2013; Keidar, 2015). Conversely, low El has been associated with anxiety, depression, posttraumatic stress disorder (PTSD) (G. J. Taylor, 2001) and borderline personality disorder (Stuss \& Levine, 2002). 
Together, these findings suggest that El skills play an important role in helping people to manage conflict and experience positive emotions in their relationships with others, while a lack thereof may lead to emotion dysregulation and possibly mental disorders. Importantly, research shows that El comprises skills that can be modified. This idea gains support from previous studies suggesting that scores on El-related measures tend to improve with age (Derksen et al., 2002; Hemmati et al., 2004; Kafetsios, 2004), as well as with training in other domains (e.g., music lessons; see (Thompson et al., 2004)). That is, these results reinforce the idea El is set of psychological skills for which expertise can be gained through sufficient practice/experience.

\section{Challenges of Current El Research}

A major challenge faced by psychology researchers studying $\mathrm{El}$ is inconsistent measurement, and therefore definition, of El's constituent constructs. The major limitation of self-report inventories that ask people to evaluate their own El abilities (i.e., recognizing, thinking about, and modifying the emotions of self and others) is what they report may not match their true abilities (Austin et al., 2004). For example, some people who rate their own social sensitivity highly receive much lower ratings from third-party observers (Carney \& Harrigan, 2003). Another approach to measuring El involves performance-based assessments, but these too, have issues. The performance of the participant is scored by "experts", yet it is theoretically unclear how such emotion experts can 
be reliably identified. There are also valid concerns regarding the age, gender, ethnicity of a chosen group of experts and how that may interact with the demographic characteristics of a given test-taker. In addition, the observed relationship between self-reported trait El and actual performance ability (e.g. recognizing emotion in faces tends to be weak ( $r=0.20$ to 0.30 ) (Austin et al., 2004; Brannick et al., 2009). A third, and more widely used approach, is consensus scoring, whereby correct answers are chosen by emotion researchers (Kafetsios, 2004). A well-recognized limitation, however, is that since the correct answer is defined by what most people choose, it can't be used for difficult questions that most people would get incorrect. Additionally, they do not correlate with other established measures of the ability to recognize emotions from facial images or voice tones (Roberts et al., 2001) or predict differences in the first impressions people make on others while working together (Day \& Carroll, 2004), as might be expected of an El test. Also, their ability to predict measures of wellbeing and life satisfaction are either greatly reduced or completely removed after controlling for other measures, such as general intelligence (IQ) tests and personality inventories (Gannon \& Ranzijn, 2005).

Since the processes that comprise El remain inconsistently defined and make its investigation problematic, a potential solution is combining subjective EI measurements with objective neuroimaging tasks, therefore testing which sociocognitive processes underlie El. Unlike the self-report methods or expert/consensus scoring methods currently employed, a neural understanding 
of El offers the possibility of a more objective means of scoring/evaluating $\mathrm{El}$ potentially allowing for a (currently lacking) performance-based neurobiological cognitive test of El.

\section{Linking El to Neuroarchitecture}

Despite its importance in navigating complex socioemotional situations in daily life, El's neurobiological mechanisms have not been uncovered. Existing neuroimaging studies of healthy individuals suggest that several brain regions may be of particular importance for the interconnected set of sociocognitive skills underlying $\mathrm{El}$, such as the orbitofrontal cortex (OFC), dorsolateral prefrontal cortex (dIPFC), amygdala, insular cortex, anterior cingulate cortex, superior temporal gyrus (STG), and visual association cortex - regions underlying emotion perception, understanding and regulation (Bar-On et al., 2003; Hornak et al., 2003; Killgore \& Yurgelun-Todd, 2007; Tarasuik et al., 2009; Xiang et al., 2017). However, leading models of large-scale brain architecture suggest that there is not a 1-to-1 mapping between brain regions and psychological functions. That is, most brain regions appear to be involved in a myriad of psychological processes, the success of which depend on the correct temporal and regional recruitment facilitating their interaction (Anderson, 2014).

Present influential models define El as an 'array of non-cognitive abilities', a problematic assertion since research suggests emotional abilities largely interact with cognitive systems (Crocker et al., 2013). In order to better 
understand how El's interconnected facets may arise from the brain's functional architecture, common "emotional" and "cognitive" mental processes related to El

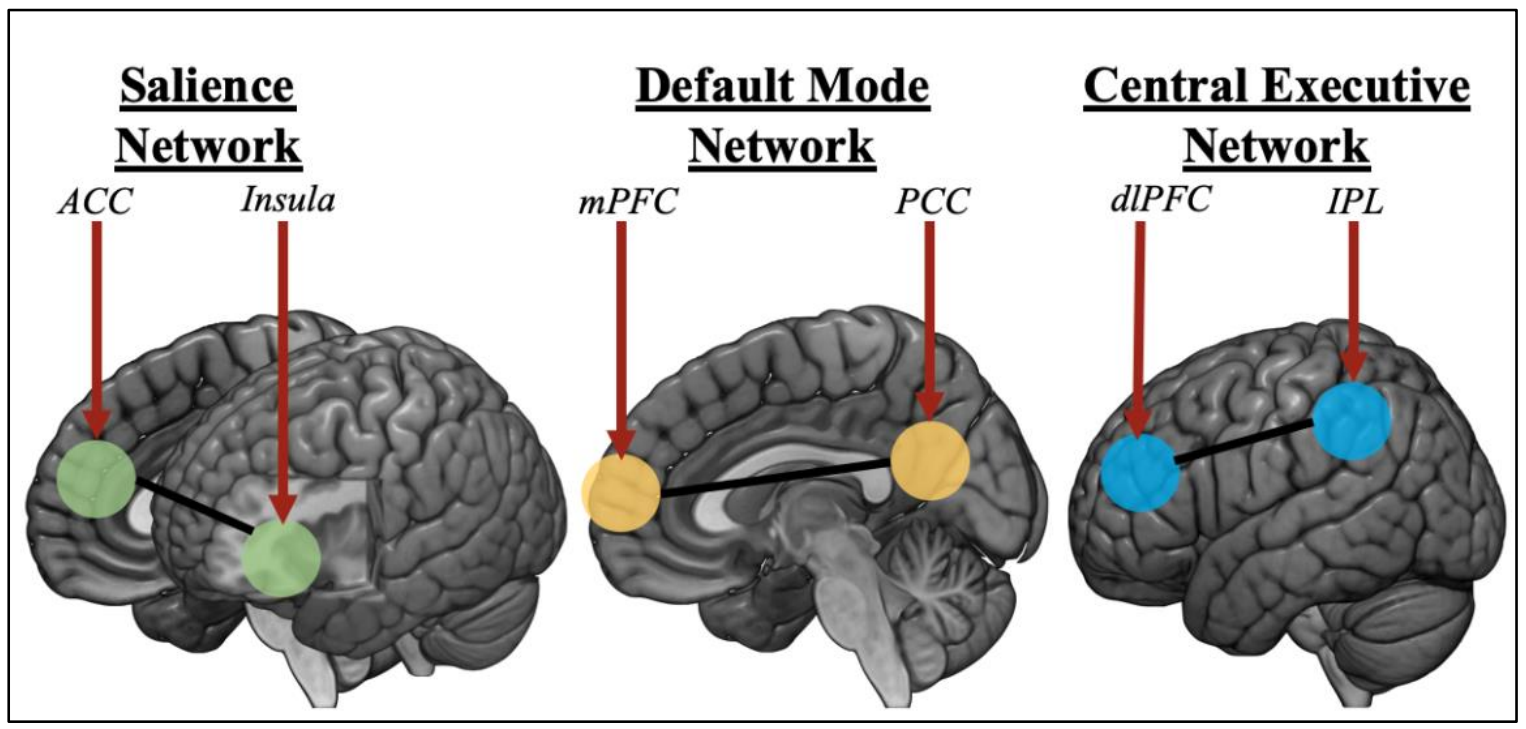

derived from a broad psychological literature will be discussed in the context of canonical intrinsic connectivity networks (ICNs). ICNs are a set of established cortical networks presumed to underlie sensory, emotional and cognitive functions (Seeley et al., 2007). For the purposes of this illustration, the salience (SN), default mode (DMN) and central executive networks (CEN) will serve as a relevant demonstration (Figure 1).

Figure 1: The Salience, Default Mode and Central Executive brain networks. Abbreviations: ACC - Anterior Cingulate Cortex, mPFC - Medial Prefrontal Cortex, PCC - Posterior Cingulate Cortex, dIPFC - dorsolateral Prefrontal Cortex, IPL - Intraparietal Lobule.

The SN includes the anterior insula (Al), anterior cingulate cortex (ACC) and in addition the dorsal cingulate and sensorimotor cortex, and features extensive connectivity with limbic structures involved in reward and motivation (Barrett \& Satpute, 2013; Barrett \& Simmons, 2015; Craig, 2009; Ondobaka et 
al., 2017; Owens et al., 2018; Seth, 2013; Yeo et al., 2011). The SN is thought to integrate information regarding bodily changes and direct attention to homeostatically/emotionally relevant stimuli (Menon, 2015; Seeley et al., 2007; K. S. Taylor et al., 2009). The DMN comprises the medial prefrontal cortex (mPFC) and posterior cingulate cortex (PCC) regions, and is thought to underlie autobiographical, self-monitoring and sociocognitive functions (Raichle, 2015; Raichle et al., 2001; van den Heuvel \& Hulshoff Pol, 2010). The CEN is a frontoparietal network with subcortical coupling distinct from the SEN and is engaged in higher-order cognitive and attentional control. The CEN is anchored in the dorsolateral prefrontal cortex (dIPFC) and inferior parietal lobule (IPL) (Chan et al., 2008; Seeley et al., 2007).

\section{Neural Correlates of Emotional El Processes}

Regarding emotional processes related to El, empathy - the tendency to share and understand others' inner lives has been characterized to involve regions anchored in the SN (Zaki, 2014). A further delineation has been made between a basic emotional contagion system, affective empathy - the sharing an emotional experience with another (I feel what you are feeling); and cognitive empathy - imagining the situation from another's perspective (I understand why you feel that way) (Shamay-tsoory, 2015). Because affective empathy has been linked to automaticity relative to its cognitive component, it primarily elicits activations from the $\mathrm{SN}$ regions implicated in rapid and prioritized processing of 
emotion signals (insula, ACC, sensorimotor cortex), coupled with limbic regions (amygdala and hypothalamus) (Decety et al., 2013). By comparison, cognitive empathy additionally involves the DMN regions coupled with the superior temporal sulcus (STS), temporoparietal junction (TPJ), and fusiform face area (FFA) (Saxe \& Powell, 2006), regions underlying conscious determination of intent. Thus, brain regions involved in empathy are associated with receiving/monitoring sensory data, subjective emotional experience and understanding complex social interactions.

Closely related to empathy, the ability to accurately identify emotions in the self and others requires self-awareness and understanding of another's perspective. Although behaviorally distinct, neurobiologically the two processes rely on similar neural mechanisms. Studies have demonstrated that regions that anchor the DMN (plus the insula and amygdala (Vogeley et al., 2001)), respond during the recognition/awareness of one's own emotional states (interoception) (Gavazzi et al., 2017). Research on emotional recognition/awareness in others reveals involvement of the $\mathrm{DMN}$ and $\mathrm{SN}$ through two important neural mechanisms: the construction of a simulation of the observed emotion in the perceiver via amygdala, STS, TPJ and FFA, followed by the top-down modulation of sensory cortices, involving the vmPFC and insula (Adolphs et al., 2000). The involvement of STS and TPJ, which are posited to contain mirror neurons (sensitive to biological motion) (Iriki, 2006), suggest these areas are specifically activated during the conscious determination of intent. As such, 
emotion recognition/awareness in the self relies on regions involved in interoception, while emotional recognition/awareness in others involves regions associated with emotion perception and categorization.

While identifying and understanding emotions in ourselves and others is necessary for $\mathrm{El}$, emotion regulation (ER) - how a person can effectively manage his/her emotions, is also critical. Research shows individuals high in El regulate their emotions successfully when necessary but do so flexibly, thereby leaving room for emotions to emerge (Peña-Sarrionandia et al., 2015). Moreover, promising research suggests El training can improve ER outcomes (Bagheri et al., 2017). Neurobiologically, ER is thought to be mediated by neural circuits involving regions anchored in the CEN: the dIPFC and vmPFC, which putatively down-regulate the amygdala (Davidson et al., 2000; Etkin et al., 2015). The dIPFC is recruited to maintain a higher-order goal representation online, while the vmPFC updates specific emotional outcome expectancies, subsequently influencing emotion regulation through direct projections to the amygdala. Thus, ER relies on brain regions involving regulation of emotional arousal in order to 
solve emotional conflict. A neural representation of the three "emotional" processes is illustrated below (Figure 2).

Figure 2: El Emotional Processes

\section{Neural Correlates of Cognitive El Processes}

In concert with "emotional" processes, processes underlying "cognition", or executive functions (EF), have been widely interrogated. A breadth of research points to the generally accepted mechanisms of decision-making, response inhibition and directed attention. Decision-making permits short-term

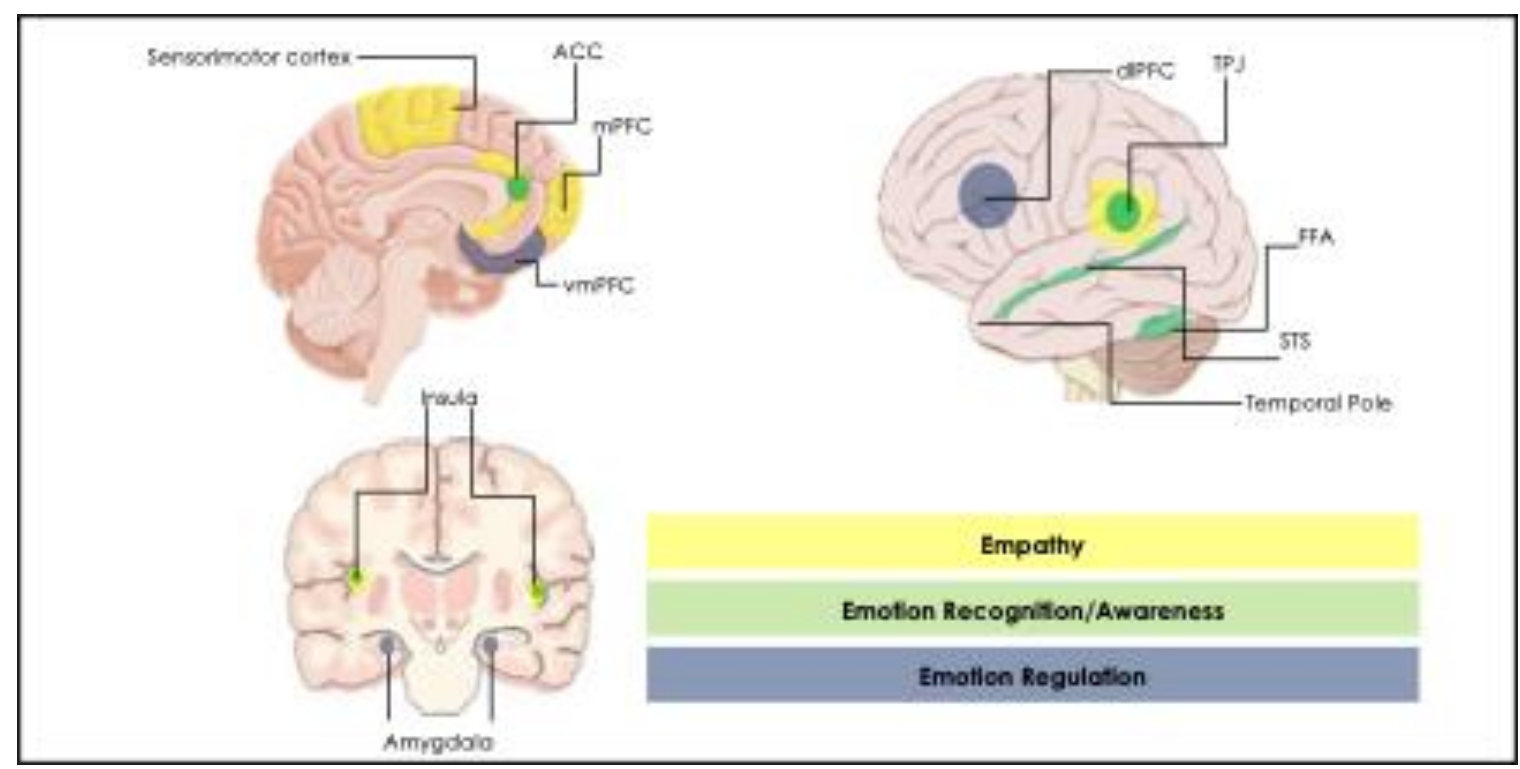

maintenance and manipulation of information while calculating reward

contingencies. Neuroimaging studies probing decision-making which relies on working memory, observe robust activation of the CEN regions vmPFC and dIPFC during memory search and maintenance processes (Barbey et al., 2013; D'Esposito et al., 1999; X. Li et al., 2010; Linden, 2007). The vmPFC also 
establishes a link between past decisions and affiliated emotions (Bechara et al., 2000), thereby aiding future decision-making. Meanwhile, vmPFC activation is observed during processing of reward contingencies, increasing the likelihood that cognitive operations are maintained by the dIPFC, thus illustrating the importance of communication between the vmPFC and dIPFC (Barbey et al., 2011; Kringelbach, 2005; Schultz et al., 2000; Tremblay \& Schultz, 1999). Thus, decision-making relies on neural circuits involving keeping goals in mind while assessing their reward expectancy.

Response inhibition, the ability to suppress inappropriate or unwanted actions, recruits a right-lateralized CEN network formed by prefrontal-parietal (rIFG-rIPL) circuits, ACC and the pre-supplementary motor area (SMA) (Simmonds et al., 2008). Putatively, the rIFG elicits top down control to suppress basal ganglia output to the pre-SMA while the rIPL keeps the location of the attention task set in mind (Corbetta \& Shulman, 2002; Rubia et al., 2001). Thus, response inhibition engages the right CEN network over planned motor actions.

Directed attention employs regions within the SEN and CN: the superior parietal lobule (SPL), IPL, dIPFC/vIPFC, and frontal eye fields (FEF). Research suggests when focusing attention selectively, communication from the dIPFC and SPL up-regulates activation in visual cortex for increased processing of contextual information related to goal attainment (Barbey et al., 2013). The vIPFC and IPL are recruited to source-monitor the general context for salient information and update behavior via connections between dIPFC and VIPFC in 
order to ensure goal attainment (Corbetta \& Shulman, 2002; Kastner et al., 1998; Mesulam, 1981). A neural representation of the three "cognitive" processes is illustrated below (Figure 3).

Figure 3: El Cognitive Processes

Critically, research suggests a connection exists between various "emotional" and "cognitive" El processes. For example, emotional information can have a positive and negative effect on decision-making and working memory maintenance (Lindström \& Bohlin, 2011; Osaka et al., 2013) and can either

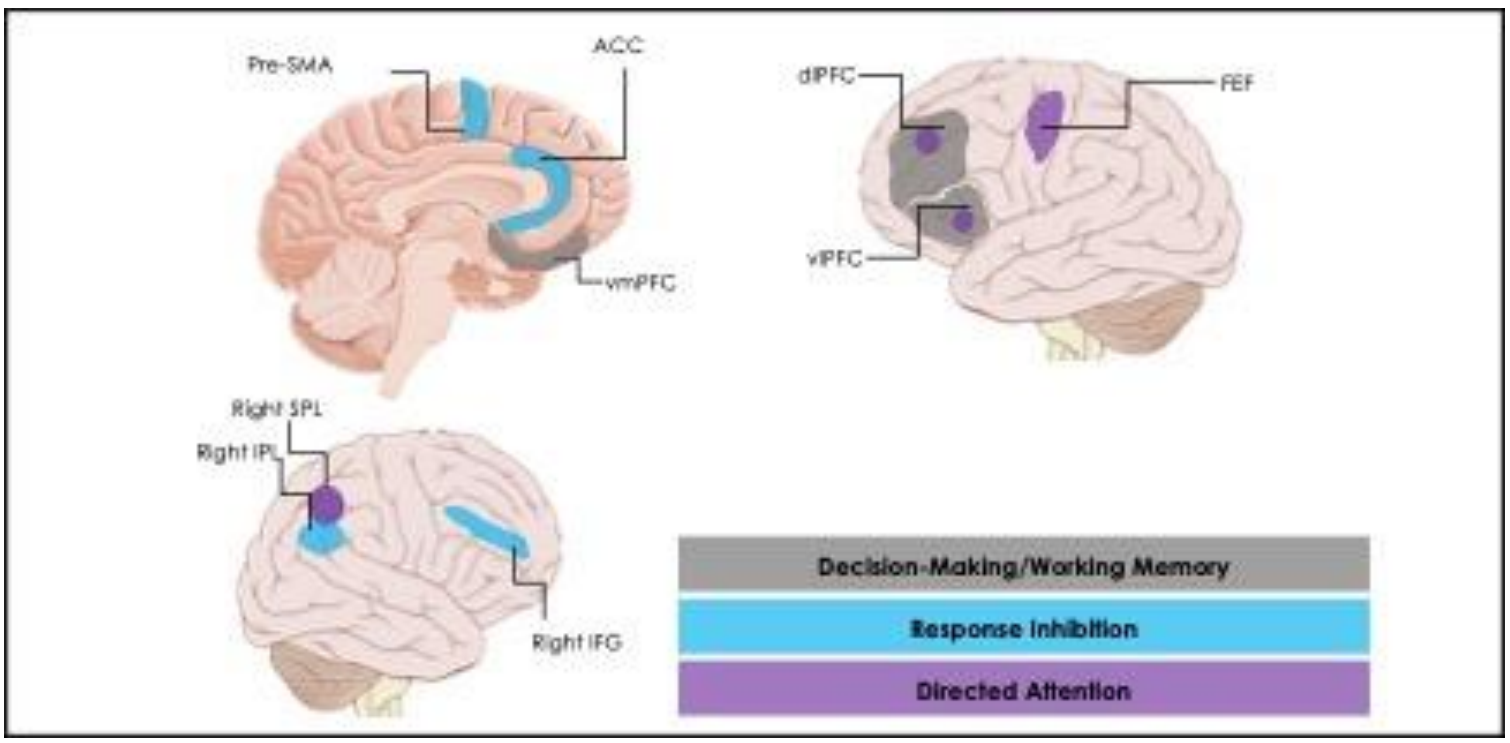

enhance or impair response inhibition depending on saliency of the stimulus (Pessoa et al., 2012). Increased attention is shown to be connected to the emotional attributes of a stimuli (Schuett, 2016), and interoception has been previously tied to important El cognitive processes such as problem solving and flexibility of thought (Mahler K, 2015). The involvement of the ACC in both executive control and emotional processing suggests this structure contributes greatly in interactions between the emotional and cognitive system (Mueller, 
2011). While many studies have either investigated emotional and cognitive EI processes separately, how the two interact with each other to create unified emotionally intelligent behavior is uncertain.

\section{Project Motivation}

The extent to which the aforementioned El processes are related, both behaviorally and neurobiologically, is presently unknown, despite the established role El plays in harmonious socioemotional communication. Due to the inconsistent El measurement, a clear El definition of its processes is lacking. Furthermore, neuroimaging studies thus far have only investigated sociocognitive processes underlying El separately, without exploring their interaction.

Understanding their interaction may explain how different ICNs work in tandem to give rise to El ability. Thus far, no study has aimed to amend this disparity by constructing and testing a cognitive-emotional El framework based on its putative neurobiological mechanisms. Therefore, the primary goals of these dissertation studies are a) to define common El processes based on their consistency in current models in order to build a cognitive-emotional El framework for testing the neural mechanisms underlying El (Chapter II), b) to test the cognitiveemotional El framework by investigating how brain networks underlying resultant El processes interact dynamically (Chapter III), c) and to explore the relationship of resultant El processes with physiological and psychological variables using a variety of brain network analyses (Chapters IV and V). 
To comprehensively investigate common El processes, we assessed how resultant El processes interact, and how this interaction is reflected to functional neuroarchitecture. This was achieved by implementing a novel neuroimaging methodology called dynamic functional connectivity (dynFC). Similar to continuous shooting mode on a camera, dynFC captures several snapshots of network interactions over the course of the scan, thereby revealing reoccurring patterns of dynFC between networks. Comparing dynFC measurements can clearly delineate how brain networks underlying El processes communicate not only spatially but also temporally. Then, each resultant El process was examined individually, to understand how they relate to other physiological variables (hormones, interoception), psychological traits (anxiety, worry, rumination) and whether any gender differences emerge, through a combination of neuroimaging methodologies (structural measurements, functional activation, static functional connectivity). Thus, this dissertation aims to answer 1) How do El processes interact on a temporal scale? 2) Are there physiological variables and psychological traits related to El processes? 3) Do men and women differ in their practice of El processes? In this way, this work will improve the understanding, cognitive/behavioral measurement, and use of the concept of El in two ways. First, it divides up the processes/abilities contributing to El in ways that are informed by the neural sciences. This holds the promise of reducing confounds as well of designing assessments that are potentially capable of offering specific information regarding where a deficit in El might originate in a given individual. It 
can also guide in the interpretation of neuroimaging results, potentially allowing such methodologies to also be of some use in identifying particular El processing deficits and also in assessing the meaning of brain changes after El training. 


\title{
CHAPTER II: BUILDING A COGNITIVE-EMOTION EI FRAMEWORK
}

"Every emotion has a source and a key that opens it."

\section{Rumi}

\begin{abstract}
Aim
In order to address the first aim of redefining common El processes and build a behavioral framework for testing the neural mechanisms underlying $\mathrm{El}$, existing trait and ability El questionnaires were correlated with measurements and tasks interrogating El "emotion" and "cognitive" processes: Decision Making/Working Memory, Response Inhibition, Directed Attention and Empathy, Emotion Recognition/Awareness and Emotion Regulation (Figure 4).
\end{abstract}

Figure 4: El processes investigated for Cognitive-Emotional EI Framework

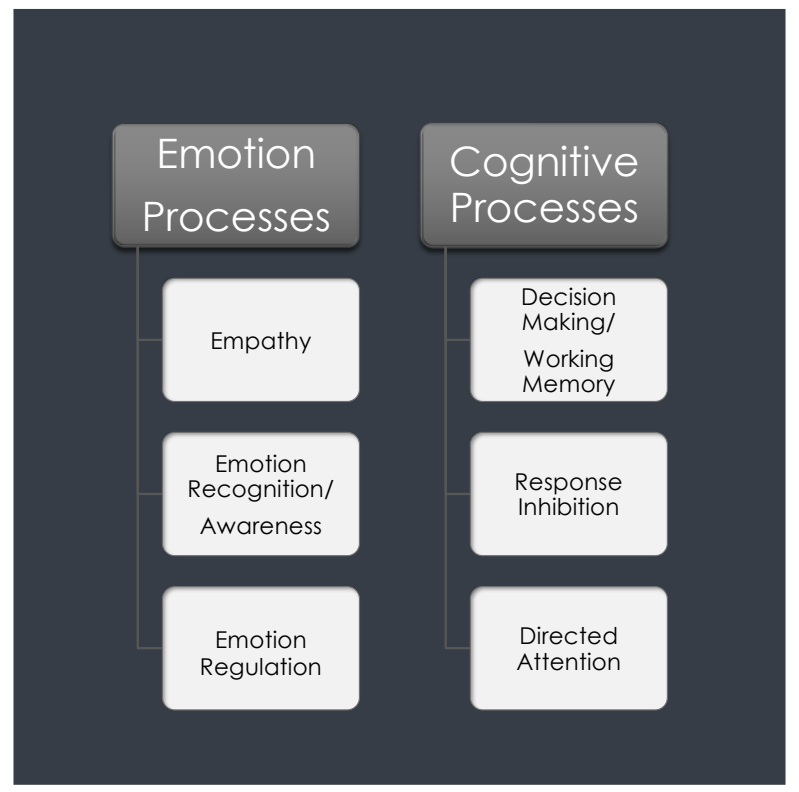




\section{Hypotheses}

We anticipate that the correlational analysis will reveal relationships between El measurements and Response Inhibition and Emotion Regulation, since both constructs require a level of control: one over movement, and one over emotion, respectively. In addition, we anticipate Empathy will be related to Directed Attention, since understanding another's emotional state requires successfully shifting the attention to another.

\section{Methods}

\section{Participants}

Participants were recruited through on-campus flyers and an online research participation system (SONA Systems) and were paid for their participation. Every effort was made to recruit an equal number of male and female subjects in each study, and to ensure that minorities were represented in proportion to the composition of the local community. 30 healthy young adults without a reported history of neurological or psychiatric disorders were recruited for this study Written informed consent was obtained prior to experimental sessions, and experimental protocols were approved by University of Louisville's Institutional Review Board prior to data collection. 3 participants were excluded 
from the analyses due to incomplete data, leaving a total of 27 participants $(\mathrm{m}=$ 21.85 years old/16 females).

Procedure

The collection of data was divided into two consecutive days. The first day, participants visited the laboratory to sign consent forms, read through task instructions and complete self-report questionnaires and tasks in a quiet room by themselves. On the second day, the participants continued completing the remainder of self-report questionnaires and tasks. A mix of "emotion" and "cognitive" tasks/questionnaires was presented each day, and the order of the tasks was optimized to manage participant fatigue (Table 1).

\begin{tabular}{|l|l|}
\hline Day 1 & Day 2 \\
\hline Ino-No-Go Task (Response & ER Task (Emotion Regulation) \\
\hline RME Task (Empathy) & $\begin{array}{l} \\
\text { lowa Gambling Task (Decision- } \\
\text { Making) }\end{array}$ \\
\hline IRI Questionnaire (Empathy) & STROOP Task (Directed Attention) \\
\hline SSEIT Questionnaire (Trait EI) & \\
\hline
\end{tabular}

\section{Stimuli}


The stimuli for the Emotion Regulation Task consist of neutral and negatively valenced pictures taken from the International Affective Picture Series (Lang et al., 2005). The stimuli for the Reading the Mind in the Eyes (RME) consist of black and white pictures of eye pairs taken from revised RME pen-and-paper test (Baron-Cohen et al., 2001).

\section{"Emotional" El Tasks and Questionnaires}

Empathy

The RME task requires participants to view black and white pictures of pairs of eyes, then choose the emotion of the individual's eyes from four choices. Interpersonal Reactivity Index (IRI)

The IRI measures affective, cognitive and total empathy and consists of 28 items rated on a 5-point scale with the anchors: "does not describe me well" to “describes me very well” (Davis, 1983).

Electronic Levels of Emotional Awareness Scale (eLEAS)

The eLEAS is a performance measure that assesses an individual's ability to be aware his or her emotions, as well as other's emotions. The scale poses evocative interpersonal situations and elicits open-ended descriptions of the emotional responses of self and others which are scored using specific structural criteria applied to the emotion words used in the responses (Chhatwal \& Lane, 2016).

\section{Emotion Regulation}


(1) Baseline ER Task - Participants rate neutral and negative IAPS pictures on a 1-4 Likert scale for how negative it makes them feel, providing a baseline measurement of ER.

(2) ER task-The task requires participants to view negative IAPS pictures within two conditions: 1) Suppress condition - participants view negatively valenced pictures and are instructed to "passively view the picture and remove yourself from any attached feeling" or 2) Feel condition- participants view negative pictures and are instructed to "Feel the emotion associated with the picture". Fifteen different IAPS pictures appear in each condition. The color of a border around the pictures indicates the condition: blue for feel trials and red for suppress trials.

(3) ER rating - Participants are asked to rate the pictures that were presented in the ER task on negative emotional feeling using a 1-4 Likert scale, which provides a behavioral measure of ER.

(4) Strategy Questionnaire - At the end of each scanning session, the participants see the ER task pictures again and write whether they used any emotion regulation strategy during the "suppress" trials. Emotion Regulation Questionnaire (ERQ)

To measure differences in habitual emotion regulation, participants completed the emotion regulation questionnaire (ERQ) (Gross and John 2003). The test measures the tendency to use cognitive reappraisal or expressive suppression to downregulate negative emotion. 


\section{"Cognitive" El Tasks and Questionnaires}

\section{Decision-Making/Working Memory}

During the virtual lowa Gambling Task (IGT), participants are given four decks of cards and an endowment of fake money (\$2000). Participants are presented with four virtual decks of cards on a computer screen. They are told that each deck holds cards that will either reward or penalize them, using game money. The goal of the game is to win as much money as possible. The decks differ from each other in the balance of reward versus penalty cards. Thus, some decks are "bad decks", and other decks are "good decks", because some decks will tend to reward the player more often than other decks (Bechara et al., 1994).

\section{Response Inhibition}

During the Go/NoGo Task, series of letters are presented in a continuous stream and participants perform a binary decision on each stimulus. One of the outcomes requires participants to make a motor response (go), whereas the other requires participants to withhold a response (no-go) (Donders, 1969).

\section{Directed Attention}

During the STROOP task, participants are required to select the written color names of the words independently of the color of the ink (for example, they would have to select "purple" no matter what the color of the font). The Stroop effect is the delay in reaction time between congruent and incongruent stimuli (Stroop, 1935).

\section{Emotional Intelligence Tests}


Trait Emotional Intelligence Questionnaire (TEIQue)

This is a 30 -item questionnaire designed to measure global trait emotional intelligence (Cooper \& Petrides, 2010).

\section{Mayer-Salovey-Caruso Emotional Intelligence Test (MSCEIT)}

The MSCEIT is an ability-based test designed to measure the four branches of the El model of Mayer and Salovey. MSCEIT consists of 141 items and takes 3045 minutes to complete. MSCEIT provides 15 main scores: Total El score, two Area scores, four Branch scores, and eight Task scores. In addition to these 15 scores, there are three Supplemental scores (Mayer et al., 2003).

Data was correlated using the corr.test function in the psych package of $R$ (Core R Team, 2019; Revelle, 2010), and corrected for age, gender and multiple comparisons using False Discover Rate (FDR) (Benjamini \& Hochberg, 1995). The correlational network was made using the qgraph package in $\mathrm{R}$ (Epskamp et al., 2012).

\section{Results}

Relationships between measurements of interest are represented in the correlational network below (Figure 5). 


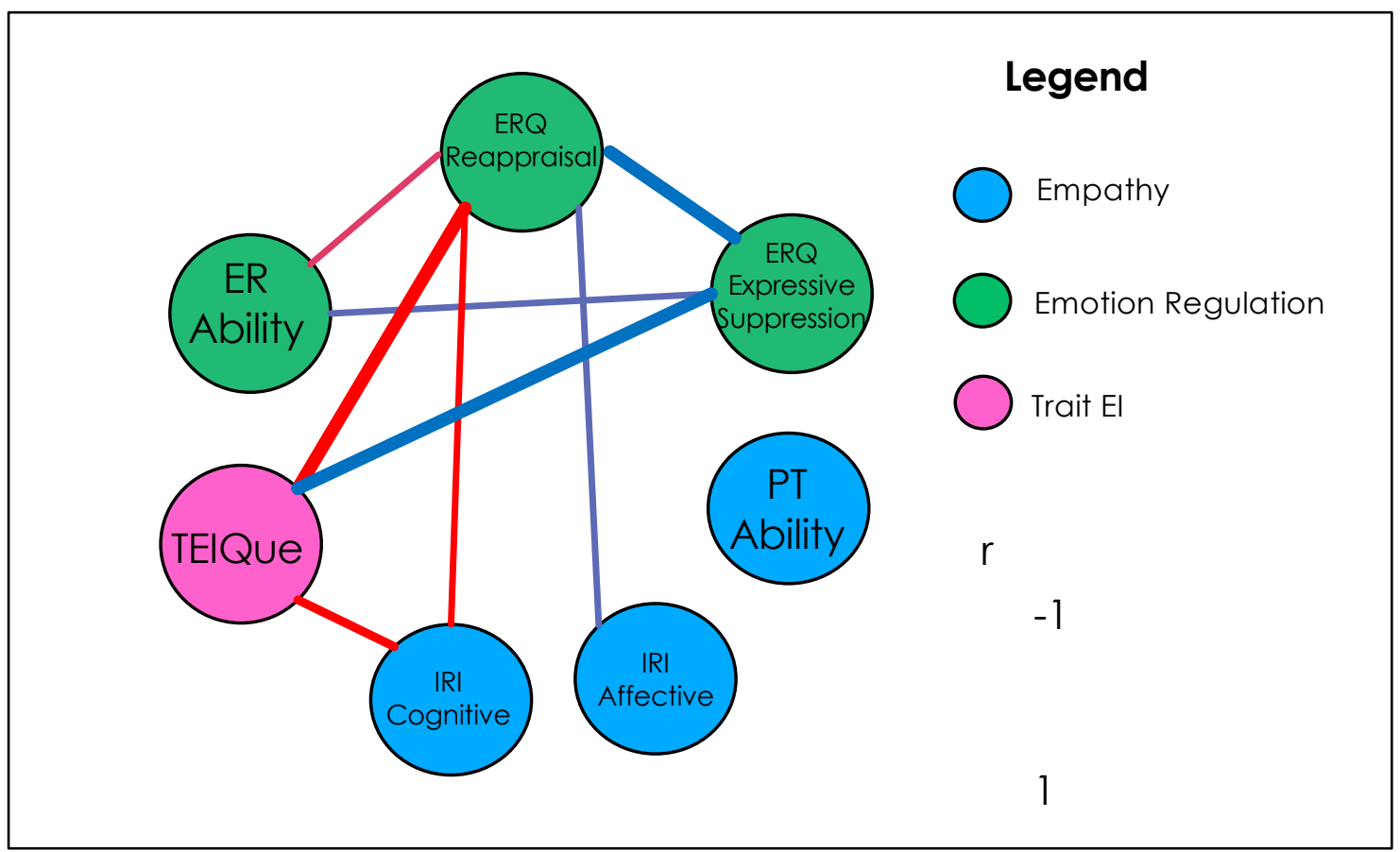

Figure 5: Correlational Network Results. Correlations corrected for age, gender and multiple comparisons (FDR). Red lines denote positive correlations, blue lines denote negative correlations. Width of line proportional to correlation strength.

We observed that ability to control emotions during a task (ER ability) was positively related to self-reported cognitive reappraisal tendency (ERQ Reappraisal), $r(23)=.50, p=.01$, but negatively related to self-reported expressive suppression tendency $r(23)=-.45, p=.02$. In addition, increased tendency to cognitively reappraise was related to less ability to share emotion with another (IRI Affective Empathy), $r(23)=-.45, p=.02$, but more ability to understand another's emotions (IRI Cognitive Empathy), $r(23)=.43, p=.03$. Lastly, increased trait EI (TEIQue) was related to more ability to understand another's emotions, $(r(23)=.46, p=.02)$, increased tendency to cognitively reappraise $(r(23)=.65$, 
$p<.01$ ), but inversely associated with tendency to expressively suppress emotions $r(23)=-.53, p=.01$.

Taken together, these behavioral findings suggest that in our sample, the constructs that best predict emotional intelligence are empathy and ER. Although traditionally thought of as "emotional" processes, brain imaging research suggests they both rely on brain regions subserving "cognitive" processes. For example, effective ER relies on the elegant orchestration of the dIPFC and vmPFC to downregulate the amygdala, yet utilization of these prefrontal regions is also exhibited in working memory and directed attention tasks (Barbey et al., 2013; Depue et al., 2016). Furthermore, empathy involves activation of the ACC and sensorimotor cortex, as does effective response inhibition (Decety et al., 2013; Rubia et al., 2001). Therefore, it is reasonable to posit that a cognitive-emotional El framework involves a combination of brain networks involving both "emotional" and "cognitive" processes.

Using these behavioral findings as a scaffold, we constructed a neuroimaging study whereby participants completed the questionnaires and fMRI tasks that showed significant relationships within our model. Resultant relationships between questionnaire scores, behavioral performance on the fMRI tasks and a variety of functional brain measurements thus serve as the neurobiological foundation of the proposed cognitive-emotional El framework. 


\section{CHAPTER III: EMPATHY AND EMOTION REGULATION}

"It seems the essence of our lives sometimes comes down to the feeling of another life against our fingers, palms, our own hands “

\section{Linda Hogan}

\section{Experiment 1: Empathy and Emotion Regulation Task Dynamics}

Empathy lies at the core of harmonious socioemotional communication. This multifaceted construct plays a critical interpersonal and societal role, enabling sharing of experiences, needs and desires between individuals, and establishes an emotional bridge that promotes prosocial behavior (Riess, 2017). Relatedly, the ability to regulate emotion is equally important during empathic socioemotional encounters to facilitate communication. Current research suggests empathy involves two distinct facets: affective empathy, or sharing an emotional experience with another (I feel what you are feeling); and cognitive empathy, or imagining the situation from another's perspective (I understand why you feel that way) (Shamay-tsoory, 2015). However, these facets have been shown to be dissociably disrupted in psychiatric illness (Cox et al., 2012). For instance, psychopathy is characterized by deficits in affective but not cognitive empathy, while conversely, autism disorder is associated with impairment in 
cognitive but not affective empathy (Cox et al., 2012). Additionally, it's common that individuals afflicted with either disorder exhibit deficits in emotion regulation (ER) ability.

The ability control one's own emotional state (James J. Gross, 1998) or ER, is posited to interact with empathy. For instance, when a parent is trying to comfort an upset child, empathy allows an understanding of the child's emotional state. However, if the parent lacks regulation over their emotions, it may result in added distress and impede the parent from appropriately responding to their child's needs. Therefore, ER ability has a critical role in empathy, the core assertion being that individuals with a lower ability for ER experience higher levels of personal distress and lower levels of empathy when observing another's negative emotional state (Decety \& Moriguchi, 2007; Zaki \& Ochsner, 2012). Yet despite their theorized interaction, no substantial evidence exists linking empathy (nor its facets) and ER ability. Clues from clinical populations suggest that in addition to being characterized by empathy deficits (Baron-Cohen \& Wheelwright, 2004; Decety \& Moriguchi, 2007; Mullins-Nelson et al., 2006), individuals with psychopathy and autism also exhibit decreased ability to regulate emotion, leading to dysregulated mood (Kim et al., 2000; Konstantareas \& Stewart, 2006; Samson et al., 2012). Unraveling how affective and cognitive empathy relate to ER ability neurobiologically may illuminate the nature of these shared deficits within clinical populations. 
Previous research suggests brain regions underlying empathy and ER are anchored in ICNs that interact together to facilitate interpersonal communication. To illustrate using the example above, to show affective empathy, the parent must be able to recognize the child's emotional experience and reflect that inward (Simone G. Shamay-Tsoory et al., 2009). This process should influence one's internal physical states, which in turn should correlate with activity in the neural structures that make up SN (Craig, 2009; Wiens, 2005), the ICN thought to be involved in sensitivity to external stimuli and the integration of sensory information (Menon, 2015; Seeley et al., 2007). A parent's increased recruitment of the $\mathrm{SN}$ is exhibited in order to observe external signals from the child, subsequently influencing the parent's physiological state, which should elicit neural activity within their DMN (Craig, 2009; Wiens, 2005), the ICN which is associated with autobiographical, self-monitoring and sociocognitive functions (Raichle, 2015; Raichle et al., 2001; van den Heuvel \& Hulshoff Pol, 2010), behaviors necessary for both affective and cognitive empathy (De Waal \& Preston, 2017). In order for an effective parent-child interaction, the negative emotion the parent recognizes and feels through the aforementioned processes must be efficiently regulated. In order for this to occur, the parent's CEN becomes more active to ameliorate unwanted negative emotional reactivity (Ochsner et al., 2002), an ICN engaged in higher-order cognitive and attentional control (Chan et al., 2008; Seeley et al., 2007). However, it is not clear how the 
various ICNs underlying the facets of empathy and ER ability interact dynamically to create harmonious socioemotional communication.

To date, nascent research has only examined the link between empathy or ER ability and static network expression (Bilevicius et al., 2018; Pan et al., 2018) that is: people's subjective empathic response or ER ability and their brain communication at a single time point. Although investigating which ICNs are related to empathy and ER ability is useful, it is insufficient for understanding their flexible temporal network (re) configurations. Elucidating how communication between brain networks underlying empathy and ER unravels across multiple points in time would illustrate how El behavior arises from their dynamic interaction.

The dynamic interactions between ICNs have been revealed by recent advances in $\mathrm{fMRI}$ analytical methods and are referred to as dynamic functional connectivity (dynFC) (Allen et al., 2014; Hutchison et al., 2013). Similar to continuous shooting mode on a camera, dynFC captures several snapshots of

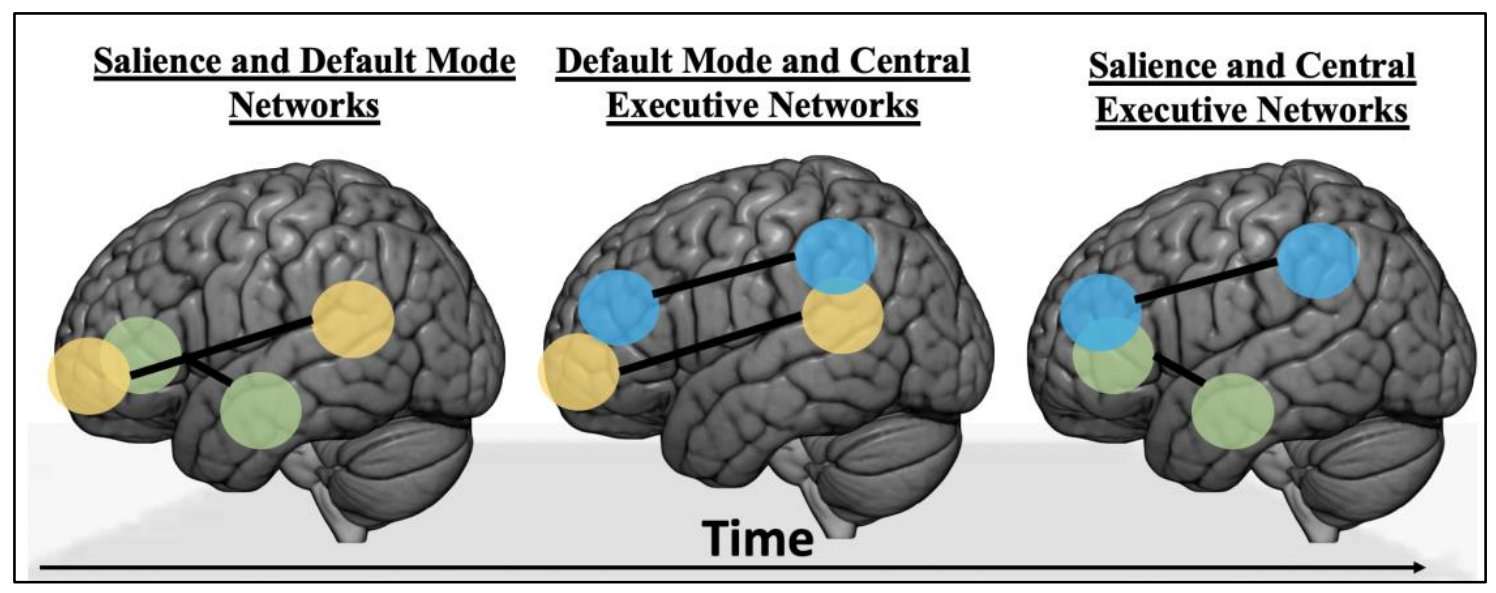


network interactions over the course of the scan, thereby revealing reoccurring patterns of dynFC between networks, called brain states (Figure 6).

Figure 6: Example of a Brain State

Importantly, dynFC brain states have been suggested to correspond to mental states (Gonzalez-Castillo et al., 2015). Comparing differences in brain state measurements related to affective and cognitive empathy, and their relation to ER ability, for example in mean dwell time (how long subjects spent in a given brain state), can clearly delineate how these processes vary not only spatially but also temporally.

Aim

Therefore, using dynFC, we aim to explore how each empathy facet (affective/cognitive) contributes to ER ability, and in a complementary investigation, explore how ER tendency contributes to empathic ability.

\section{Hypotheses}

We anticipate increased affective empathy will relate to higher mean dwell time in brain states expressing SN and DMN network positive connectivity, and that increased cognitive empathy will relate to higher mean dwell time in brain states expressing CEN positive connectivity and DMN negative connectivity. We also expect increased ER ability and affective empathy will relate to higher mean dwell time in brain states expressing CEN and DMN positive connectivity, as well as decreased ER ability and cognitive empathy will relate to higher mean dwell 
time in brain states expressing SN positive connectivity and CEN negative connectivity.

\section{Methods}

All participants were required to answer an MRI screening questionnaire to ensure their safety in an MR environment. In addition, participants were at least 18 years of age, right-handed, native English speakers, with normal or corrected-to-normal vision and hearing and had no disclosed history of neurological or psychiatric disorders. These exclusion criteria are standard in neuroimaging research to reduce potential confounds due to handedness, differences in perceptual abilities, or effects of psychiatric drugs. Participants were screened for being a native English speaker as there may be difficulty in interpreting task instructions as a result of language. Every effort was made to recruit an equal number of male and female subjects in each study, and to ensure that minorities were represented in proportion to the composition of the local community. Recruited participants were fully informed and made as comfortable as possible in order to maximize retention rates. Candidate subjects responding to these notices received a brief description of the research and completed prescreening questions over the phone. When arriving to participate in a study, participants were familiarized with the protocol by the experimenter, including risks and benefits of the research. In the case of fMRI sessions, participants also completed a detailed screening form to indicate any contraindications based on a superset of the Society for Magnetic Resonance in 
Medicine standardized MRI screening protocol (absolute exclusions for ferrous metal in any part of body, such as pacemakers, cochlear implants, surgical clips or metal fragments, serious medical conditions, claustrophobia). To protect against potential risks of boredom, fatigue, or frustration, participants were allowed rest breaks as needed. Participants' comfort levels were monitored throughout the session. Participants could communicate with the experimenter at all times. It was made clear that participation is voluntary and that participants could withdraw from the study at any time without penalty or prejudice. Any questions that the subjects had were answered by the experimenter. After testing, participants were debriefed as to the purpose and predictions of the experiments. Written informed consent was obtained prior to all experimental sessions, and experimental protocols were approved by University of Louisville's Institutional Review Board prior to data collection.

fMRI Tasks

(1) Reading the Mind in the Eyes (RME)

The Reading the Mind in the Eyes task is a measure of "mentalizing," or reading the mind state of another. The participant is presented with a series of $30 \mathrm{~B} \& \mathrm{~W}$ photographs of the eye-region of the face of different Caucasian actors and actresses and is asked to choose which word best describes what participant is 
thinking or feeling from the list of adjectives. Each photograph and choice are displayed for 6 seconds (Figure 7).

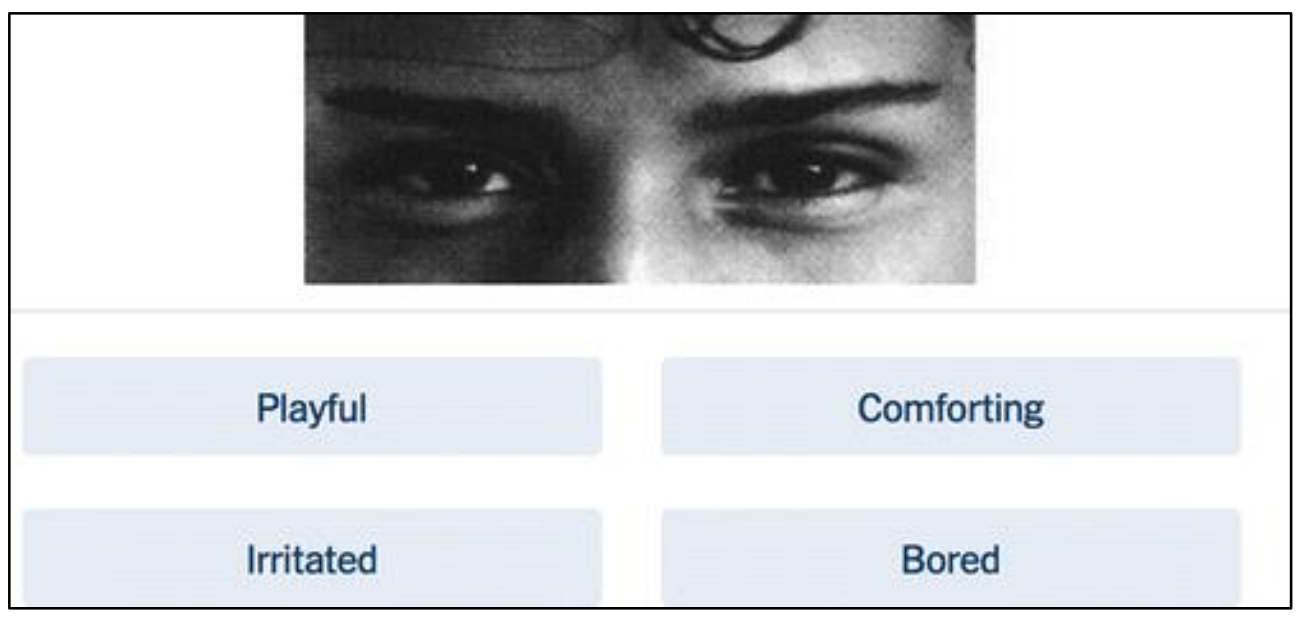

Figure 7: Example trial from RME task.

(2) Emotion Regulation Task (ERT)

The ERT was divided into three parts: (A) ER Baseline (B) Emotion Regulation (ER) (C) ER Rating. Only parts A \& B were BOLD scans, part C was structural. (A) ER Baseline:

The ER Baseline task employed a hybrid event-related design that contained mini-blocks presented in pseudorandom order. 20 negative International Affective Picture System (IAPS) pictures and 10 neutral pictures were displayed (Lang et al., 2005). The pictures were displayed for 4 seconds. This design was chosen to balance considerations for the psychological state of the participant with statistical power. Following presentation of each stimulus, participants rated the image for how negative it made them feel, using a 4-point Likert scale ( $1=$ None to 4 = Extremely negative; 2 seconds to rate; Figure 8). This allowed acquisition of a subjective negative baseline rating for each participant. A pseudorandom 
variable jittered ITI was incorporated to increase design efficiency for hemodynamic response estimation $(0-10 \mathrm{sec})$. Resultant behavioral ratings from the task were further used to calculate ER Suppression Score (see part C).

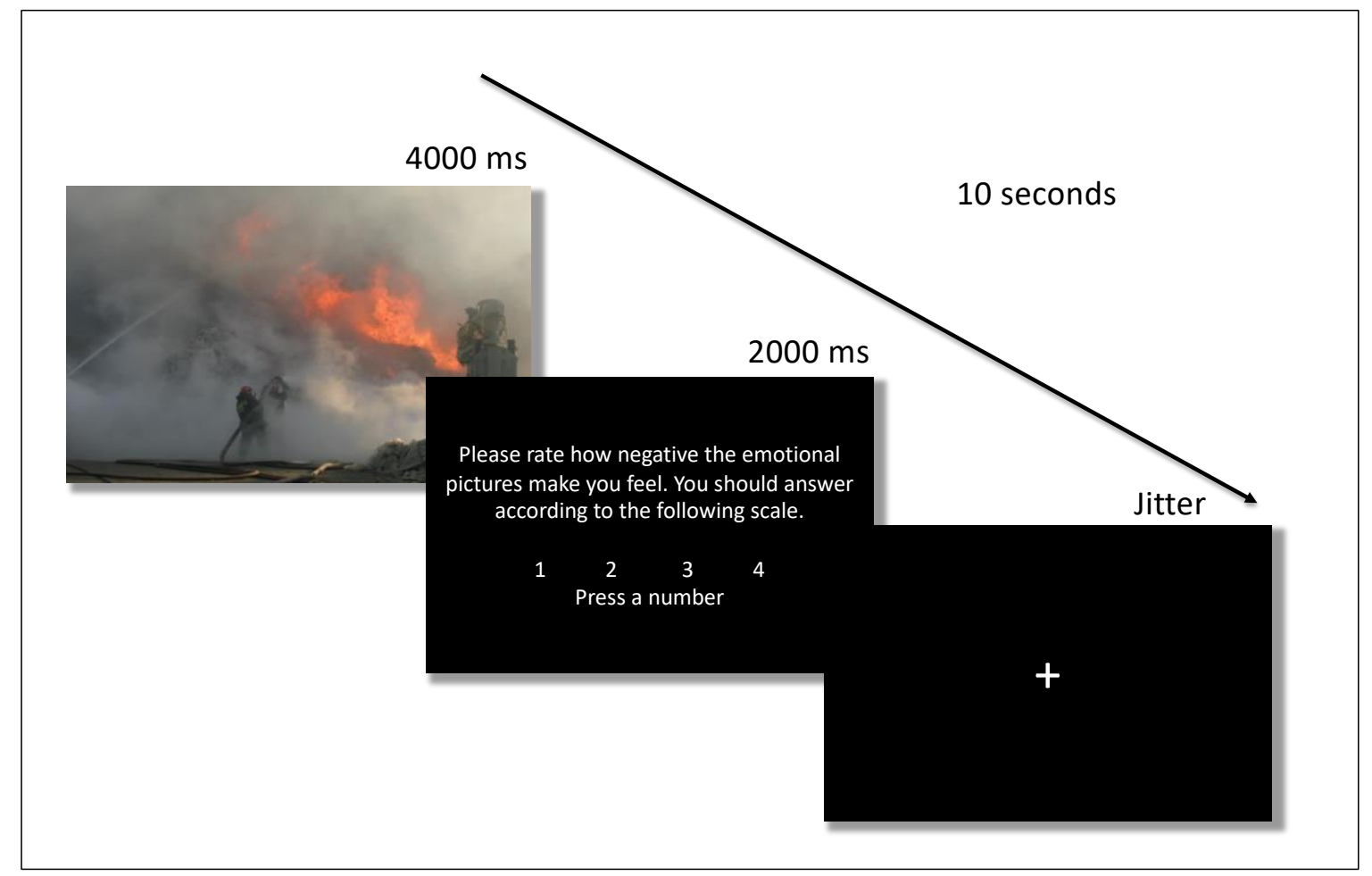

Figure 8: Example of a single trial from ER Baseline Task (Image displayed not part of actual stimuli set)

(B) Emotion Regulation (ER):

The ER task also employed a hybrid event-related design with mini blocks presented in pseudorandom order, whereby a different set of negative and neutral pictures selected from the IAPS database were displayed (Lang et al., 2005). First, the words 'SUPPRESS' in the color red or 'VIEW' in the color blue appeared as cues for $500 \mathrm{~ms}$ to prepare the participant for the upcoming picture. Next, negative pictures surrounded by a red border (Suppress trials) and neutral 
pictures surrounded by a blue border (View trials) were presented for 3.5 seconds (Suppress trials $n=30$, View trials $n=12$ ) (Figure 9). The participant was instructed to ' decrease or detach' from the emotion when the border was red, and 'simply view' the picture when the border was blue". The "Suppress" instructions were worded simply in order to encourage the participant to use their default ER method. A pseudorandom variable jittered ITI was incorporated to increase design efficiency for hemodynamic response estimation $(0-4 \mathrm{sec})$. The

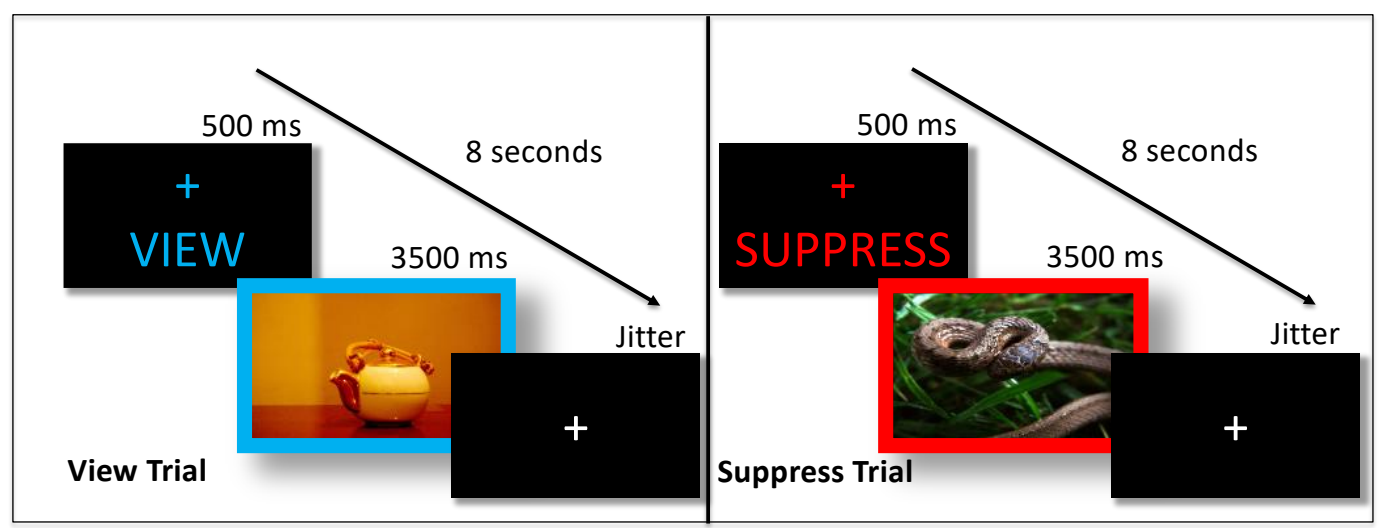

pictures repeated once, with a 60 second break in-between.

Figure 9: Example of a single trial of each condition from Emotion Regulation (ER) (Image displayed not part of actual stimuli set).

(C) ER Rating

During a structural scan, the same images used in part B were then displayed without any border for 4 seconds. Following presentation of each image, participants rated how negative the image made them feel, using a four-point Likert scale $(1=$ None to $4=$ Extremely negative, displayed in the same way as $E R$ Baseline). The negative ER ratings from this part were subtracted from the earlier acquired negative baseline ratings (part A) in order to calculate a 
Suppression Score. The Suppression Score was derived from negative ratings only (rather than ratings on "View" trials) to ensure an accurate measurement of negative affect down-regulation success (i.e. comparing a response to negative stimuli before and after ER). The Suppression Score was used as an independent variable in subsequent FC analyses which served as the dependent variable.

Imaging Data Acquisition

All structural MRI images were acquired using a Siemens 3-T Skyra MR scanner. A 20-channel head coil was used for radiofrequency reception. Participants were given earplugs to reduce scanner noise and were additionally given headphones to receive instructions. Foam padding was added to limit motion if additional room remained within the head coil, and a piece of folded tape was placed over the participant's forehead as a reminder to remain still throughout the scan. Structural images were obtained via a T1-weighted magnetization-prepared rapid gradient-echo sequence (MPRAGE) in 208 sagittal slices. Imaging parameters were as follows: echo time $(T E)=2.26 \mathrm{~ms}$, repetition time $(\mathrm{TR})=1,700 \mathrm{~ms}$, flip angle $=9.0^{\circ}$, field of view $(\mathrm{FoV})=204 \mathrm{~mm}$, and voxel size $=0.8 \times 0.8 \times 0.8 \mathrm{~mm}$. Functional blood oxygenation level-dependent $(B O L D)$ images were collected using gradient-echo $\mathrm{T} 2^{*}$-weighted echoplanar imaging $\left(\mathrm{TE}=28 \mathrm{~ms} ; \mathrm{TR}=2000 \mathrm{~ms} ;\right.$ flip angle $=79^{\circ} ; \mathrm{FoV}=204 \mathrm{~mm} ;$ voxel size $=3.2$ $\mathrm{mm}^{3}, 38$ interleaved slices). Slices were oriented obliquely along the AC-PC line. Imaging Data Preprocessing 
Preprocessing of $\mathrm{fMRI}$ data was performed using the CONN Functional Connectivity Toolbox 20.b (Whitfield-Gabrieli \& Nieto-Castanon, 2012) based on SPM12 (Penny et al., 2007) in the $2020 \mathrm{~b}$ version of MATLAB. All preprocessing steps were conducted using the default preprocessing pipeline for volume-based analysis (to MNI-space). First, each subject's functional images were realigned to the first volume and unwarped (which implements the removal of dynamic EPI distortions, movement-by-susceptibility interactions as described in http://www.fil.ion.ucl.ac.uk/spm/toolbox/unwarp/), slice-timing corrected (interleaved bottom-up), co-registered with structural data, spatially normalized into the standard MNI (Montreal Neurological Institute) space, and finally images were smoothed using a Gaussian kernel of $6 \mathrm{~mm}$ FWMH. Moreover, noise was reduced via the anatomical CompCor approach (Behzadi et al., 2007), which extracts principal components from white matter and cerebrospinal fluid time series. The six head motion parameters from the ART toolbox were also included as a confound regressors. Next, a [0.008 to $\infty]$ temporal band-pass filter standard for task-based connectivity analyses was applied to the time series (NietoCastanon, 2015). Linear detrending was additionally performed. In sum, detrending, outlier censoring, motion regression, and CompCor correction were performed simultaneously in a single first-level regression model, followed by band-pass filtering. These corrections yielded a residual BOLD time course at each voxel that was used for subsequent analyses.

Group ICA 
After preprocessing the data, Group ICA of fMRI Toolbox (GIFT) was used to decompose the data into functional networks using group spatial independent component analysis (ICA) (Calhoun et al., 2001). First, subject-specific data was reduced to 29 independent components (ICs) (as estimated by the minimum description length (MDL) algorithm) with the principal component reduction as previously done (Allen et al., 2014; Damaraju et al., 2014). To ensure stability and validity, we repeated the Infomax ICA algorithm in ICASSO 20 times (Himberg et al., 2004). Aggregated spatial maps were estimated. The back reconstruction approach (GICA) was used to obtain subject-specific maps and time courses as implemented in the GIFT toolbox (Calhoun et al., 2001). After visual inspection by independent reviewers, spatial correlation values between the $19 \mathrm{ICs}$ and the consensual atlas of resting-state networks (CAREN) based on 4 freely available brain functional Atlases (Doucet et al., 2011, 2018; Gordon et al., 2016; Yeo et al., 2011) were used for ICs selection, according to these 5 categories: Visual (VIS) Salience (SN), Sensorimotor-Auditory (SMN), Default Mode (DMN) and Central Executive Networks (CEN) (Doucet, 2019). Components were classified as intrinsic connectivity networks (ICNs) if they exhibited peak activations in grey matter, had moderate correlation values with time courses of the "Suppress" (ERT) and "Rate" (RME) task conditions (above 0.3 ) and strong correlation values with the CAREN network template (above 0.50) (Cordes et al., 2001). After ICs selection, subject-specific spatial maps and time courses were post-processed, following (Allen et al., 2014), and included a detrending (3) and despiking $(0.15 \mathrm{~Hz})$. 


\section{Dynamic Functional Connectivity Analysis (DynFC)}

Dynamic FC (dynFC) analysis was performed with the GIFT toolbox. The 22 TR sliding window method for each subject was applied following (Allen et al., 2014), whereby overlapping time windows of 30 s were taken from the scanning time in steps of $2 \mathrm{~s}(1 \mathrm{TR})$ and convolved with a Gaussian of sigma $=3$ TRs in order to de-weigh volumes at the beginning and end of the windows, resulting in 240 consecutive windows for the ERT and 157 consecutive windows for the RME. The length of the window $(22 \mathrm{TRs}=44 \mathrm{~s})$ was based on previous studies in the field because it provides a good trade-off between the ability to capture dynamic changes and the accuracy of correlation estimation (Biundo et al., 2015; Cilia et al., 2011; Hutchison et al., 2013). For each window, dynFC was estimated in the form of a regularized inverse covariance matrix using the GIFT toolbox using graphical LASSO method with an additional L1 norm constraint, repeated 10 times. Finally, covariance estimates were Fisher-Z-transformed.

In order to identify dynFC states that reoccurred across time and across subjects, the windowed dynFC matrices were subjected to the GIFT k-means clustering procedure, repeated 100 times to reduce any bias to initial random selection of cluster centroids (Rashid et al., 2014). K-means clustering applies Euclidean distance to regroup similar dynFC matrices of the different windows. The number of clusters (k), or states, can be calculated in several ways. In this study we used the elbow criterion, defined as the ratio of within-cluster distances to between-cluster distances, following previous dynFC studies (Allen et al., 
2014; Damaraju et al., 2014) and the cluster number was set to 5 for the ERT and 4 for the RME. Next, we used the Pearson correlation coefficient for the clustering analysis. To sum up, a subset of windows was selected for each subject, representing those dynFC matrices with maximal variability in dynFC. From those windows, the optimal number of clusters $(k)$ was determined by the GIFT toolbox using the elbow criterion. The resulting $k$ cluster centroids were used as templates for clustering all the dynFC windows of matrices of all subjects. Using the resultant four cluster centroids, all FC matrices of each subject were then categorized as belonging to one of the four states based on their similarity to the cluster centroids. From these data, we obtained a state transition vector representing their state status across time. Final cluster centroids were obtained as the median of all state-assigned FC matrices across time. The subject-specific centroid of each state was computed by calculating the median value of each FC matrix for that state.

Finally, indexes from dynFC were extracted. They were: 1) Mean dwell time, which represents how long participants stayed in a certain state, which was calculated by averaging the number of consecutive windows belonging to one state before changing to the other state and 2) Number of transitions, which represents how many times either state changed from one to the other, counting the number of times a switch occurred, with more transitions representing less stability over time. To investigate how empathy trait is reflected in the dynFC of brain networks underlying emotion regulation, ERT dynFC indexes were 
correlated with IRI scores. Conversely, to assess how ER tendency is reflected in the dynFC of brain networks underlying empathy, RME dynFC indexes were correlated with $E R Q$ scores. Correlations between the extracted dynFC measurements and questionnaires were assessed in the Statistical Package for Social Science (SPSS) (IBM SPSS Statistics 24), controlling for age, gender multiple comparisons using FDR (Benjamini \& Hochberg, 1995).

\section{Results}

\section{ERT Dynamics Regressed with IRI Empathy Questionnaire}

Following the elbow criterion, the sample showed 5 different dynFC states during the ERT. Indexes from 2 dynFC states had significant relationships with behavioral measurements and will be described in detail below.

Dynamic Functional Connectivity State 1 
State 1 (13\% of the windows) was characterized primarily by positive connectivity between the CEN, DMN and SN (Figure 10).

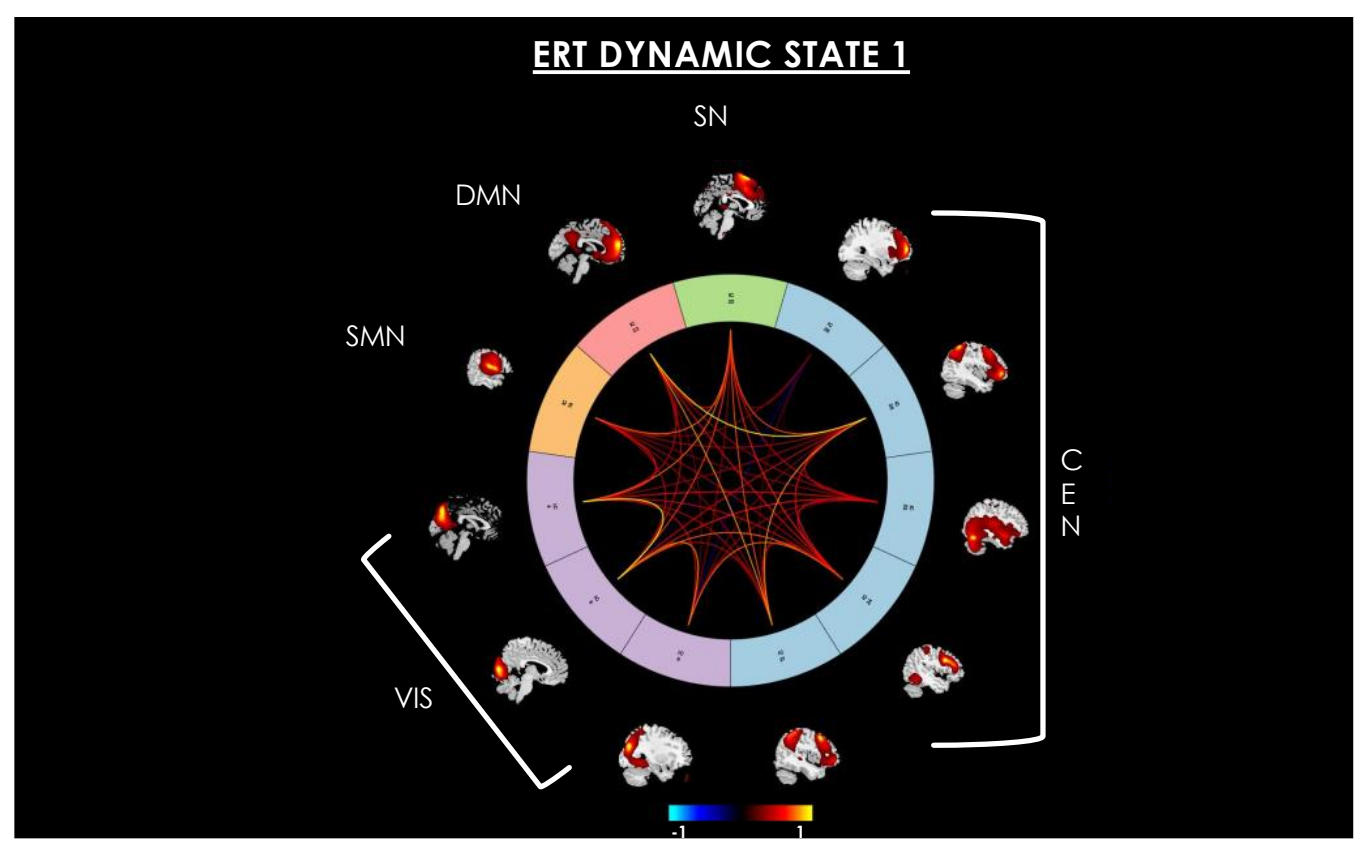

Figure 10: Dynamic Functional State 1 of the ERT

Amount of time spent in this state (mean dwell time) exhibited a positive relationship with IRI Affective Empathy $r(22)=.51, p=.01$. No other behavioral measurements exhibited significant relationships with this state's dynFC indexes. Dynamic Functional Connectivity State 2 
State $2(19 \%$ of the windows) was characterized by positive connectivity between the CEN and the SN and SMN, and negative connectivity between the VIS network and the CEN, SMN, DMN, SN (Figure 11).

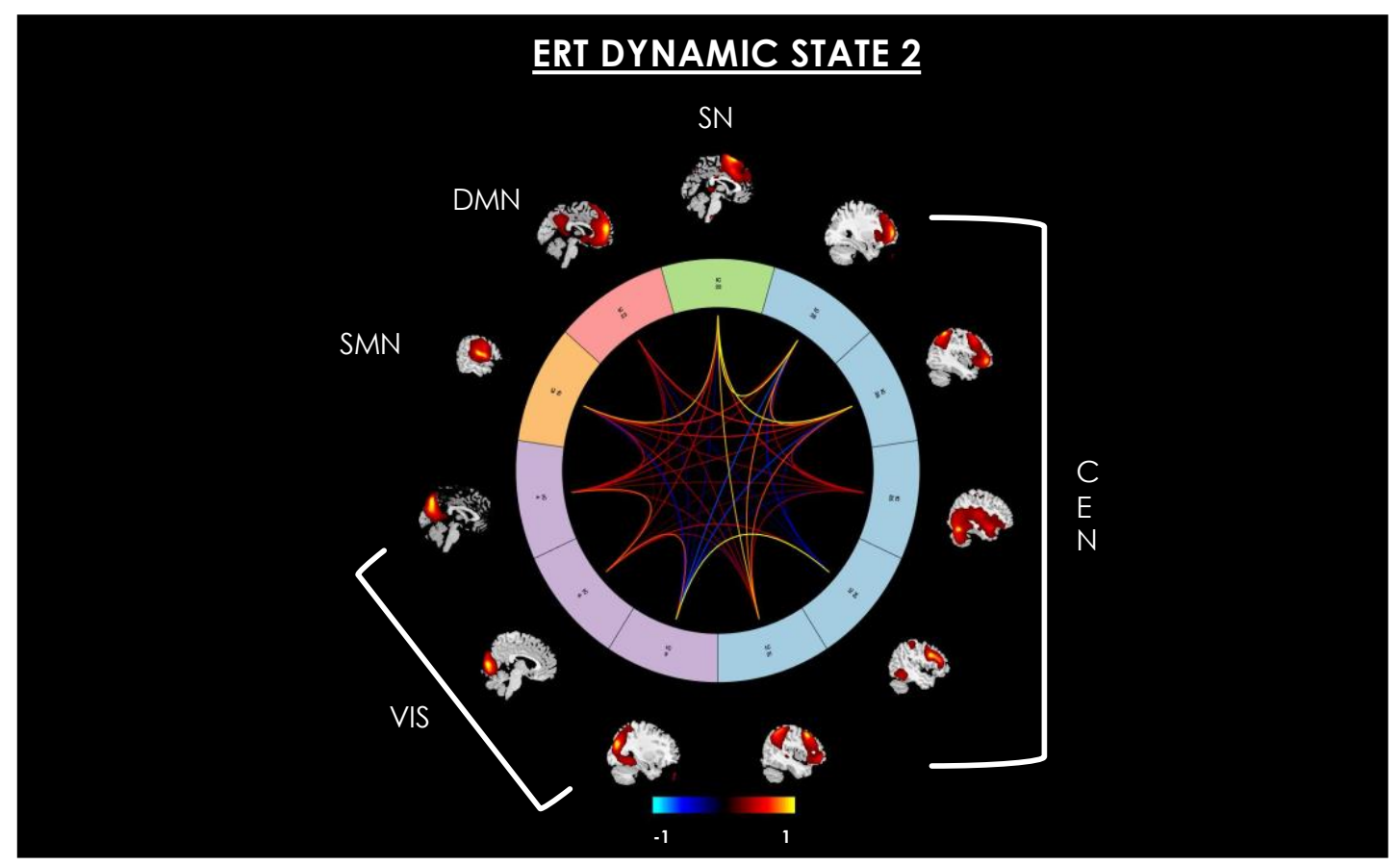

Figure 11: Dynamic Functional State 2 of the ERT

Amount of time spent in this state (mean dwell time) exhibited a negative relationship with IRI Cognitive Empathy $r(22)=-.48, p=.02$. No other behavioral measurements exhibited significant relationships with this state's dynFC indexes. RME Dynamics Regressed with ERQ Questionnaire

Following the elbow criterion, the sample showed 4 different dynFC states during the ERT. Indexes from 1 dynFC state had significant relationships with behavioral measurements and will be described in detail below.

Dynamic Functional State 1 
State 1 (22\% of the windows) was characterized by positive connectivity between the CEN/VIS and hypoconnectivity between DMN and CEN/SMN/SN/VIS (Figure 12).

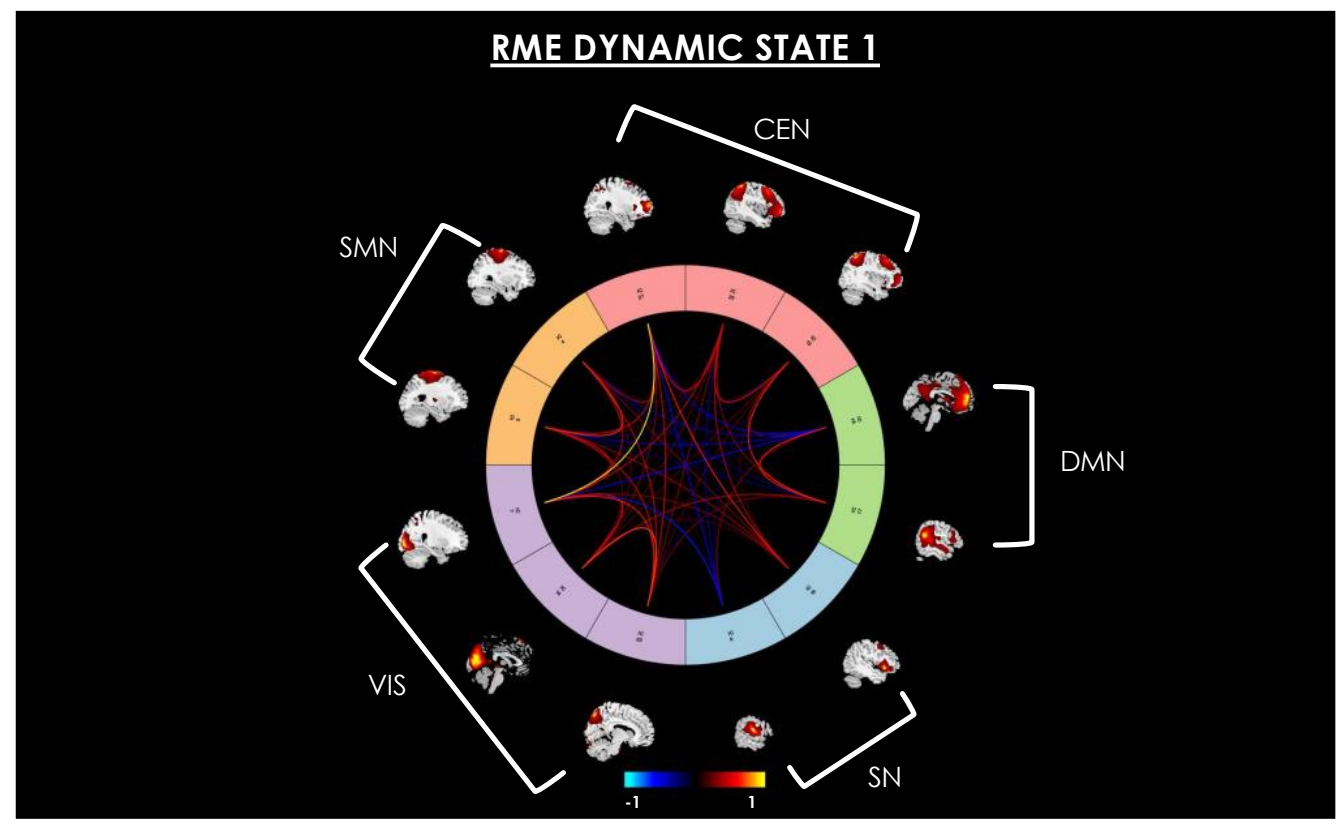

Figure 12: RME Dynamic State 1

Amount of time spent in this state (mean dwell time) exhibited a negative relationship with ERQ Cognitive Reappraisal $r(23)=-.43, p=.02$. No other behavioral measurements exhibited significant relationships with this state's dynFC indexes.

\section{Discussion}

The aim of this study was to evaluate whether dynFC during an ER task related to trait empathy, and conversely, whether dynFC during an RME task related to ER tendency. Findings suggest that the orchestrated interaction of the 
CEN, DMN and SN gives rise to these brain dynamics, and that these fluid network patterns relate to trait empathy and ER tendency.

Review of Behavioral Findings

As previously discussed in Chapter II, our behavioral findings showed inverse relationships between cognitive reappraisal and empathy, depending on the type of empathy interrogated. ERQ Cognitive Reappraisal showed a negative relationship with the affective empathy facet of the IRI, but a positive relationship with the cognitive empathy facet. The findings suggest the increased tendency to employ cognitive reappraisal to down regulate negative emotion is associated with decreased ability to share, but increased ability to understand another's emotion. Affective empathy has previously been associated with increased reactivity to others' emotions, greater spontaneous facial mimicry (T. W. Lee et al., 2008; Sonnby-Borgström, 2002), and increased self-reported resonance with the mimicked emotion (Hatfield et al., 1993; Laird et al., 1994; Strayer, 1993; Wild et al., 2001). Therefore, the heightened arousal tendency in affective empathy may result in greater interference on cognitive control, thereby reducing the individual's capacity to engage in adaptive regulation strategies such as cognitive reappraisal. In contrast to affective empathy, cognitive empathy is reliant upon higher-order cognitive control processes in order to inhibit one's default self-perspective and take another's perspective (Carlson et al., 2004; Hansen, 2011; Joseph \& Tager-Flusberg, 2004; Sabbagh et al., 2006). Given the overlap in cognitive control processes, it is possible that higher levels of cognitive 
empathy may also be associated with the efficacious use of cognitive processes that support cognitive reappraisal. Indeed, we replicate findings of a recent study that showed people with higher cognitive empathy stated they were more likely to reappraise (Powell, 2018).

Relationship between Emotion Regulation Task Brain Dynamics and Trait

\section{Empathy}

We observed that increased dwell time in first ERT dynFC brain state, characterized by positive connectivity between the CEN, DMN and SN, related to increased affective empathy. The results suggest that while down-regulating negative emotion, time spent in a state defined by synchrony between networks subserving cognitive control, autobiographical thought and sensory perception relates to increased ability to share another's emotions. Although previous research examining brain mechanisms underlying ER suggest reliance primarily on the CEN (Davidson et al., 2000; Etkin et al., 2015), we extend these findings by demonstrating ER may involve the interaction of the CEN with the $\mathrm{SN}$ and DMN, networks underlying feeling and understanding one's own emotions (Gavazzi et al., 2017; Saxe \& Powell, 2006; Vogeley et al., 2001). Indeed, these findings are in line with research suggesting effective emotion regulation may be preceded by understanding one's own feelings (C. D. Frith \& Frith, 2006), thereby possibly implicating the SN and DMN. Furthermore, our results suggest increased reliance on this combination of networks facilitates affective empathy. Even though participants only spent $13 \%$ of their time in this state, it its possible 
overreliance on these networks may prove useful in understanding how excessive affective empathy poses a risk for developing internalizing disorders, including depression and anxiety (Blair, 2005; Gambin \& Sharp, 2016, 2018; Gawronski \& Privette, 1997; Schreiter et al., 2013; Shu et al., 2017; Silton \& Fogel, 2010; Thoma et al., 2011; Tone \& Tully, 2014; Zahn-Waxler et al., 2010). We speculate that if the empathizer's emotional reaction is overwhelming, it may tax the DMN and SN to such an extent that it may lead to inappropriately modulating the shared emotional reaction (reliant on the CEN). Indeed, inappropriate modulation of emotion due to heightened arousal has been associated with internalizing symptoms (Kaźmierczak et al., 2013). Hence, the first ERT dynFC brain state may be a network configuration prominent during the downregulation of emotion, but that also underlies affective empathy ability. Increased dwell time in the second ERT dynFC brain state, characterized by positive CEN/SN connectivity and negative connectivity between CEN/VIS related to decreased cognitive empathy. Research indicates the SN and CEN may interact with each other in supporting attention and working memory (Cocchi et al., 2013; Elton \& Gao, 2014). Specifically, the SN receives and provides selective amplification of salient information and then generates a top-down control signal that initiates $\mathrm{CEN}$ to respond to salient information for attentional shift and control execution (Menon, 2015). In combination with negative connectivity between networks subserving external visual attention (CEN/VIS), this pattern of connectivity is interpreted as reflecting the internally driven goal- 
directed state. Therefore, this network configuration may arise to enable attention deployment toward goal-relevant behavior, in this case, the downregulation of negative emotion during the ERT. Our results further suggest that more time spent in this brain state was associated with decreased ability to take another's perspective. We postulate that an internally focused attention state is ineffective for successful mentalizing, since the attention should be primarily shifted outwardly, towards another person. Therefore, during a demanding (internally focused) cognitive task such as ER, there may not be enough cognitive resources to also support attention shifting towards salient external stimuli.

The present findings enrich prior ER research by demonstrating that trait empathy shares a double dissociation with brain dynamics underlying $E R$, depending on the type of empathy interrogated: increased dwell time in an ER dynFC brain state related to increased affective empathy, but increased dwell time in another ER dynFC brain state related to decreased cognitive empathy. This may be explained by one perspective of brain states, described as "the repertoire of more or less flexible brain network configurations that emerge dynamically to enable context-appropriate behavior based on the skillful interchange between external and internal needs" (Denkova et al., 2019). Therefore, the double dissociation observed between empathy's facets with ER brain dynamics may reflect deployment of attentional processes subserving 
internally driven (affective empathy) or externally driven (cognitive empathy) mental activities.

Relationship between Empathy Task Brain Dynamics and Emotion Regulation Tendency

During the RME task, we observed that increased dwell time in a dynFC brain state dominated by positive connectivity between CEN/VIS, and negative connectivity between DMN and all other networks was associated with a decreased tendency to cognitively reappraise negative emotion. Given the nature of the RME task (visual processing and decision-making), positive CEN/VIS dynFC was not surprising, given these networks support these functions (Seeley et al., 2007). Similarly, the DMN has been consistently shown to be deactivated when subjects engage in goal-directed tasks (Greicius et al., 2003; Raichle et al., 2001). Together, this pattern of connectivity is interpreted as reflecting an externally driven goal-directed state. The converse of the connectivity pattern of the internally driven state during the ER task, which showed a negative association with cognitive empathy; increased time spent in this RME dynFC state relates to a decreased tendency to use cognitive reappraisal. Cognitive reappraisal is defined as the attempt to reinterpret an emotion-eliciting situation in a way that alters its meaning and changes its emotional impact (James J. Gross \& John, 2003; Lazarus \& Alfert, 1964); and has been shown to activate regions anchored in the CEN, namely the medial and lateral PFC, which down-regulate activation of emotional arousal-related brain 
structures as amygdala and insula (Ochsner \& Gross, 2005; Zaki et al., 2012). We postulate that an externally driven goal state is ineffective for successful cognitive reappraisal, since reappraisal is an internally driven mechanism. In line with our behavioral findings that show a positive relationship between cognitive reappraisal and cognitive empathy, during a demanding (externally focused) cognitive task such as the RME, there may not be enough cognitive resources allocate attention towards a taxing (internally focused) task such as reappraisal.

\section{Summary}

In conclusion, our investigation using dynFC to probe the relationship between empathy and ER revealed increased dwell times during the ER task brain states related bi-directionally to affective and cognitive empathy, and increased dwell time during an RME task brain state related to decreased tendency to use cognitive reappraisal. Specifically, the present findings suggest that efficient communication and (re)configuration between the CEN, DMN, SN underlie both ER and RME task dynamics, and that these temporal patterns relate to trait empathy and ER tendency. Thus, we propose El may arise from the efficient interplay between brain networks subserving cognitive control, autobiographical thought and sensory perception. Taken together, these findings contribute a neurobiological basis for the hypothesized relationship between ER and empathy, and further suggest El ability may be arise from their dynamic interaction. 


\section{Limitations and Future Directions}

Several limitations of this study should be acknowledged. First, we recognize we had a small sample size, which could contribute to low power in detecting desired outcomes. Future studies with larger samples may be necessary to clarify how dynFC indexes relate to behavioral measurements. Additionally, our sample consisted primarily of Caucasian participants, and additional research should examine these questions with more ethnically and racially diverse samples. It would be interesting to study possible cultural differences relating to cognitive and affective empathy, emotion regulation, and the ways in which these constructs may interface with internalizing symptoms in other cultures. The field would benefit from longitudinal research examining these questions over time in a diverse sample of male and female individuals, especially for the RME task. In addition, performance on the RME task may differ according to sex (BaronCohen et al., 1997; Dorris et al., 2004; Losh et al., 2009). Previous studies have reported sex differences in typical individuals for neural activation associated with the RME task (Baron-Cohen et al., 2006), specifically, activation differences have been identified in the dIPFC and the medial temporal gyrus. To understand how empathy and emotion regulation differ in men and women, potential sexdifferential effects should be considered in larger samples. 


\title{
CHAPTER IV: A CLOSER LOOK AT EMPATHY
}

"Human morality is unthinkable without empathy"

Frans de Waal

\begin{abstract}
EXPERIMENT 2:
Shared Characteristics of Intrinsic Connectivity Networks Underlying Interoceptive Awareness and Empathy
\end{abstract}

Internal body signals relative to emotion processing has been a topic of long-standing interest (Gurney, 1884; Strack et al., 1988), with more recent evidence highlighting an intriguing bidirectional relationship between sensations that arise internally and emotional phenomena (Cameron, 2001; Craig, 2009; Damasio, 2005; Lane, 2008). A proposed biological basis that may clarify this interplay is interoception, namely - the afferent processing of internal bodily signals that arise from visceral organs (Cacioppo et al., 2000; Cameron, 2001; Craig, 2009; Johnson, 2001; Wiens, 2005). For example, an increased heart rate signals an emotional modulation, indicating that the assessment of one's own emotions requires interoceptive processes (K. H. Lee \& Siegle, 2012). Indeed, evidence suggests a consistent relationship between emotional experience and 
interoception (Critchley et al., 2004; Pollatos et al., 2007; Werner et al., 2009). In addition, neuroimaging findings corroborate a substantial overlap between the neural substrates of one's own emotional and interoceptive processing. This highlights the proposed idea that interoception plays an important role in emotional self-assessment (Adolfi et al., 2017; Critchley \& Garfinkel, 2017; Damasio et al., 2000; Terasawa et al., 2013). However, the relationship between signals arising from one's own body and the emotions of another individual is a topic that remains relatively unexplored.

A harmonious social interaction putatively hinges on whether the observer can vicariously feel and understand the mental state of the listener, a socioemotional ability known as empathy (Davis, 1980). Empathy can be further fractioned into two interrelated facets: Affective and Cognitive empathy. Affective empathy is conceptualized as the automatic process of vicariously experiencing the emotional state of another (Baron-Cohen \& Wheelwright, 2004; Davis, 1980), while Cognitive empathy describes the individual's ability to accurately imagine another person's perspective (Davis, 1980; Decety \& Jackson, 2004; Lawrence et al., 2006). The two facets of empathy exist on a continuum. While Affective empathy requires the empathizer to represent both 'self' and 'other', Cognitive empathy requires a marked 'self' and 'other' distinction in order to successfully imagine a different perspective from one's own (Steinbeis, 2016).

One popular interpretation of such a 'shared representation' (Decety and Sommerville 2003) posits that we represent others' experiences in terms of self- 
experience, which may explain why interoceptive awareness (IA; processed internal sensations part of conscious awareness), plays such a crucial role in social encounters (Cameron, 2001; Khalsa et al., 2018). Indeed, a substantial amount of evidence points to IA influencing the degree to which an individual experiences their emotions (Barrett et al., 2004; Wiens, 2005). For instance, those with high IA report heightened emotional arousal (Dunn et al., 2010; Pollatos \& Schandry, 2008; Wiens, 2005), which suggests better IA could lead to greater Affective empathy due to the fact the shared emotion is more intense. In addition, increased IA has also been tied to decreased susceptibility to body ownership illusions (Tajadura-Jiménez \& Tsakiris, 2014; Tsakiris et al., 2011), suggesting a clearer divergence between 'self' and 'other' which may positively relate to Cognitive Empathy.

Current neuroimaging evidence indeed suggests that the neural substrates of empathy overlap with those involved in self-experience (lacoboni, 2005; Jackson et al., 2005; Keysers et al., 2004; Wicker et al., 2003), supporting the theory that the brain represents others' experiences in terms of the experiences of the self (Decety \& Sommerville, 2003). For instance, in the Jabbi, Swart, and Keysers 2007 study, activation of the anterior insula (Al) and inferior frontal operculum (IFO) was observed in both the observer and experiencer during aversive taste stimuli. Similarly, observing others' pain has been found to robustly activate the $\mathrm{Al}$ and anterior cingulate cortex (ACC), regions associated 
with one's own pain (Jackson et al., 2005; Lieberman \& Eisenberger, 2009; Singer et al., 2004).

However, in the 'shared representation' context, it is unclear which brain regions underlying a specific aspect of empathy contribute to IA. This may be due to empathy's facets activating interacting, but only partially overlapping, neural bases (Y. Fan et al., 2011). Affective empathy primarily elicits activations from regions implicated in rapid and prioritized processing of emotion signals, including: the amygdala, hypothalamus, orbitofrontal cortex (OFC) and $\mathrm{Al}$ (Decety et al., 2013). By comparison, Cognitive Empathy, which shares similar neural networks with mentalizing and Theory of Mind (TOM) (Pardini \& Nichelli, 2009) additionally involves the superior temporal sulcus (STS), temporoparietal junction (TPJ), fusiform gyrus (FG), and medial prefrontal cortex (mPFC) (Saxe \& Powell, 2006). Thus, it is plausible to theorize that IA may share neural bases with Affective empathy within the Al and amygdala, and with Cognitive empathy within the PFC. A better understanding whether there is a disassociation between these constructs in relation to IA could therefore refine and extend the ‘shared representation' hypothesis.

Although no studies have explored the neural intersection of IA and empathy's two facets, one recent meta-analysis did investigate convergent areas of activation between IA, emotion and social cognition (Adolfi et al., 2017). The results for the three domains converged in the $\mathrm{Al}$, amygdala, right inferior frontal gyrus (rIFG), basal ganglia and medial anterior temporal lobe (mATL), ascribing 
particular importance to the fronto-temporo-insular nodes (Adolfi et al., 2017). The authors conclude co-activation of these regions may result in an evaluative association of the internal milieu, and in combination with external cues, leads to complex social cognition (Adolfi et al., 2017). However, only partial insight can be gleaned from these results in connection to the present study. The authors of the Adolfi et. al 2017 study describe the complex domain of "social cognition" simply in terms TOM (the attribution of mental states to oneself and others) (BaronCohen et al., 2001). TOM only takes into account the Cognitive and not Affective facet of empathy, and according to the Shamay-Tsoory et al. 2010 model, both are required for intact empathic processing. Therefore, within the framework of the 'shared representation' hypothesis, this meta-analysis only offers a limited glimpse into how IA and empathy's facets are neurologically related.

Nevertheless, this activation-based meta-analysis revealed a number of key brain regions known to play a role within a distributed socioemotional network. Scant functional connectivity (FC) data exists directly addressing how these regions communicate and how their communication could result in representing others' experiences in terms of the experiences of the self (Decety \& Sommerville, 2003). Thus far, one study investigating deficits in a patient with depersonalization disorder (body self-awareness disruption) employed graphtheory analyses during an empathic task and demonstrated impaired Affective empathy and IA related to changes in a interoceptive-emotional network, specifically in the Al, ACC and somatosensory cortex (Sedeño et al., 2014). 
Although germane, the study only supports an association between these domains during active, task-relevant network configurations. However, if the brain uses the 'self' as a blueprint for understanding others' emotional experiences as proposed by Decety and Sommerville 2003, it stands to reason that the brain's intrinsic connectivity networks (ICNs) during resting-state (rsfMRI) already contain the information necessary for task-based expression.

In fact, several studies corroborate this assumption. Recently Tavor et al. 2016 applied computational models showing that resting state functional connectivity (rsFC) alone is sufficient to predict individual variability in task maps, and that this pattern of intrinsic connectivity can be predictive of a subject's identity, similar to a fingerprint (Finn et al., 2015). Importantly, Bilevicius et al. 2018 illustrated that empathy scores were correlated with different patterns of rsFC in the default mode network (DMN), salience network (SN), and left and right central executive networks (CEN). Similarly, Cox et al. 2012 showed that relative empathic ability (REA) is reflected in the brain's rsFC. Lastly, ChristovMoore et al. 2020 utilized machine learning to demonstrate rsFC patterns within resonance and CEN networks can predict trait empathic concern. No evidence regarding trait IA within rsfMRI exists thus far, but studies point to a large-scale brain system supporting interoception comprising the DMN and SN (Kleckner et al., 2017). Therefore, it is possible to hypothesize that empathy and IA could share rsFC within the DMN or SN, supporting the 'shared representation' hypothesis through rsFC data. 
In addition to rsFC, BOLD variability is an often discounted neuroimaging measurement that may offer complementary information regarding network function and organization. What BOLD variability represents has been unclear, but recent neuroimaging advances suggests it may reflect network coherence throughout the cortex, and therefore a complementary reflection of functional connectivity (Fox, 2005; Mišíc et al., 2011; Vakorin, Lippe, et al., 2011). Although BOLD variability is often ignored because it has been attributed to various confounds that are deliberately minimized (in the name of improving signal-tonoise ratios) (Garrett, Samanez-Larkin, et al., 2013), several areas of neuroscience research have examined the properties and unique functionality of variance, and suggest that by considering rather than ignoring variance, our ability to understand and predict neural phenomena can improve dramatically (Faisal et al., 2008; MacDonald et al., 2006; R. B. Stein et al., 2005). In fact, recent theories consider high BOLD variability necessary for the neural system's adaptability, efficiency and cognitive performance (Dai et al., 2016; Garrett et al., 2010; Garrett, Kovacevic, et al., 2013; McIntosh et al., 2008; Vakorin, Lippe, et al., 2011; Vakorin, Mišić, et al., 2011). Specifically, according to the coordination dynamics theory, networks demonstrating increased BOLD variability can flexibly shift through integrative and segregative configurations, maintaining the neural system in balance (Tognoli \& Kelso, 2014). In the same way rsFC is used to predict task performance in individual subjects (Finn et al., 2015), resting state BOLD variability (rsBOLD) is used to show that the subject- and task specific 
BOLD variability signature is stable and persistent across time (Gaut et al., 2018). rsBOLD variability has been in clinical populations to investigate alteration in the organization of brain networks (Good et al., 2020; Kumral et al., 2020; Scarapicchia et al., 2018; Zhang et al., 2020) and in healthy populations to investigate brain maturation trajectories (Nomi et al., 2017) and degree of cognitive flexibility (Armbruster-Genç et al., 2016). Although no studies in a healthy sample have yet explored rSBOLD variability in relation to trait empathy or IA, this inquiry could shed light on how networks underlying these constructs communicate. For instance, increased rsBOLD variability in $\mathrm{SN}$ and/or DMN in relation to empathy and IA could putatively be related to effective switching between 'self' and 'other', leading to successful empathizing.

\section{Aims}

Therefore, the present study employs a data-driven approach to explore rsFC and rsBOLD variability related to brain networks underlying Cognitive, Affective empathy and IA. Specifically, we aim to understand whether Cognitive and/or Affective empathy as measured by self-report questionnaires share rsFC and/or rsBOLD variability with IA self-report measures during resting state in healthy adults.

Hypotheses 
We hypothesize based on previous literature that 1) Affective empathy will share rsFC and/or rsBOLD with IA within a SN network, specifically amygdala, $\mathrm{Al}$, and IFO, given their involvement in the processing of emotion experienced in oneself and vicariously for others (Singer et al., 2004), while 2) Cognitive empathy will share rsFC/ rsBOLD variability with IA within a mentalizing network, specifically in the rTPJ and precuneus, as these regions are posited to underlie explicit mentalizing (Bardi et al., 2017; Hyde et al., 2015; Kovács et al., 2014; Naughtin et al., 2017).

Participants and Procedure

26 healthy young adults ( $m=21.85$ y.o./16 female) without a reported history of neurological or psychiatric disorders were recruited for this study. All participants were right-handed and had normal or corrected-to-normal vision and hearing. The study took part on two separate days. On the first day, participants visited the lab to be briefed on the MRI protocol, fill out consent forms and behavioral assessments. On the second day, participants completed the rsfMRI scan at the University of Louisville, School of Medicine.

The participants watched a 7 min abstract, nonsocial movie titled Inscapes previously demonstrated to evoke strong connectivity in networks that resemble rest more than those exhibited during conventional movies (Vanderwal et al., 2015). The movie features a series of technological-looking abstract shapes. Participants were told to keep their eyes open and relax while watching and listening to the movie. The stimulus was displayed using E-prime on an Invivo 
Esys LCD TV monitor at the back of the scanner bore, which was viewed by participants through a mirror on the head-coil. The video is freely available for download from HeadSpace Studios.

Empathy Questionnaire - Interpersonal Reactivity Index (IRI)

Affective and Cognitive empathy was assessed using the Interpersonal Reactivity Index (IRI) (Davis, 1983). The IRI consists of 28 items rated on a 5point scale with the anchors: "does not describe me well" to "describes me very well". The items are arranged into four subscales with seven items. Each subscale measures a distinct component of empathy: empathic concern (EC) (feelings of compassion and concern for others); personal distress (PD) (feelings of anxiety and discomfort that result from observing another person's negative experience); perspective taking (PT) (the ability to adopt the perspectives of other people and see things from their point of view); and fantasy subscale (FS) (the tendency to identify with characters in movies, books, or other fictional situations) (Davis, 1983). Affective empathy, the ability to infer an agent's feelings or emotions, was derived from summing the EC and PD subscales. Cognitive empathy, the ability to infer an agent's beliefs or thoughts, was derived from summing the FS and PT subscales. Total empathy was derived from aggregating Affective and Cognitive empathy scores. All scores were standardized by applying a z-score transformation, and later compared with the MAIA assessment and its subscales (see below) in subsequent analyses. Multidimensional Assessment of Interoceptive Awareness (MAIA) 
The Multidimensional Assessment of Interoceptive Awareness (MAIA) is a 32-item instrument assessing IA: "the conscious perception of sensations from inside the body that create the sense of the physiological condition of the body, such as heart beat, respiration, satiety, and the autonomic nervous system sensations related to emotions" (Mehling et al., 2012). Each statement is rated from 0 (never) to 5 (always) in terms of how often it applies to the participant generally in daily life. The statements are then separated into 8 subscales: Noticing, Non-Distracting, Not-Worrying, Attention-Regulation, Emotional Awareness, Self-Regulation, Body Listening and Trusting, which are in turn aggregated into 5 overall scales used in the present study: Awareness of Body Sensations (Noticing); Emotional Reaction and Attentional Response to Sensations (Not-Distracting, Not-Worrying); Capacity to Regulate Attention (Attention Regulation), Awareness of Mind-Body Integration (Emotional Awareness, Self-Regulation, Body Listening) and Trusting Body Sensations (Trusting). A total Interoceptive Score (MAIA Total) was derived by summing all the aggregate scales. All scores were standardized by applying a z-score transformation, and later compared with the IRI and its subscales (see above) in subsequent analyses. Correlations between behavioral measurements were conducted with the Statistical Package for Social Sciences (Version 25.0.0; SPSS, INC.), and corrected for age, gender and multiple comparisons using false discovery rate $p<0.05$ (FDR) (Benjamini and Hochberg 1995). Neuroimaging Methods 


\section{Preprocessing}

All analyses were conducted using the CONN toolbox 19.c (WhitfieldGabrieli \& Nieto-Castanon, 2012) based on SPM12 (Penny et al., 2007) in the 2017 version of MATLAB. Spatial preprocessing in the CONN toolbox included realignment, normalization and smoothing ( $6 \mathrm{~mm}$ FWHM Gaussian filter) using SPM12 default parameter settings. Anatomical volumes were segmented into gray matter, white matter and cerebrospinal fluid (CSF) areas, and the resulting masks were eroded to minimize partial volume effects. Physiological and other sources of noise were estimated and regressed out using CompCor (Behzadi et al., 2007), a method that performs principal component analysis to estimate the physiological noise from white matter and cerebrospinal fluid for each participant. Motion from the ART toolbox was also included as a confound regressor. Next, a $0.01-0.10 \mathrm{~Hz}$ temporal band-pass filter standard for resting-state connectivity analyses was applied to the time series (Nieto-Castanon, 2015). In sum, detrending, outlier censoring, motion regression, and CompCor correction were performed simultaneously in a single first-level regression model, followed by band-pass filtering. These corrections yielded a residual BOLD time course at each voxel that was used for subsequent analyses.

Neuroimaging Analysis

Network Connectivity

Group-level independent component analysis (ICA) using the CONN toolbox was conducted to identify networks of functionally connected brain 
regions during resting state that may be associated with the IRI and MAIA scores. This involved the application of the fastICA algorithm to volumes concatenated across subject and resting state condition in order to identify independent spatial components (ICs) and back-projection of these components to individual subjects, resulting in maps of regression coefficients representing connectivity between the network and every voxel in the brain (see Calhoun et al. 2001 for details). Forty ICs were identified using spatial overlap of suprathreshold areas (Dice coefficient (Rombouts et al., 1998)), based on CONN's default network atlas with ROls characterizing an extended set of classical brain networks: Default Mode Network (4 ROls), SensoriMotor (2 ROls), Visual (4 ROls), Salience / Cingulo-Opercular (7 ROls), DorsalAttention (4 ROls), FrontoParietal / Central Executive (4 ROls), Language (4 ROls), Cerebellar (2 ROIs) (all ROls defined from CONN's ICA analyses of HCP dataset / 497 subjects) (Nieto-Castanon, 2014). We chose 40 ICs due to research suggesting ICA results are only affected by the number of ICs when the number is smaller than the number of source signals (Ma et al. 2007), in addition to assuring coverage of a majority of signal variance. Noise components were further identified through visual inspection by authors (TS and BD) (e.g., components largely overlapping CSF), resulting in the exclusion of 4 out of 40 ICs from further consideration. Each of the remaining $36 \mathrm{ICs}$ was subsequently entered in multiple regressions with the IRI and MAIA subscales, aggregate and Total scores. For each network, the resulting statistical maps were cluster thresholded 
at $p \leq 0.05$, voxel thresholded at $p<0.001$ (FDR-corrected) using Gaussian Random Field Theory, and corrected for age and gender. All coordinates reported below refer to peak activations in anatomical MNI space.

\section{Network Variability}

In order to assess network variability and its relationship to either empathy or IA, we regressed each IC's network variability (calculated in CONN as SD of each IC's BOLD timeseries: SDBOLD (Nieto-Castanon, 2020) with the IRI and MAIA subscales.

\section{Results}

\section{Behavioral Results}

In order to establish the relationship between empathy and IA, all IRI and MAIA subscales, aggregate and Total scores were correlated.

Relationship between Affective Empathy and Interoceptive Awareness

Excluding same-subscale correlations, negative relationships were observed between Affective empathy and the following MAIA subscales: Capacity to Regulate Attention $(r(26)=-0.83, p<0.01)$, Trusting Body Sensations $(r(26)=-0.55, p<0.01)$ and MAIA Total $(r(26)=-0.64, p<0.01)$. Similarly, we observed a negative relationship between the Personal Distress (PD) subscale and Capacity to Regulate Attention $(r(26)=-0.74, p<0.01)$, Awareness of Mind Body Integration $(r(26)=-0.49, p<0.01)$, Trusting Body Sensations $(r(26)=-0.53$, $p<0.01)$ and MAIA Total $(r(26)=-0.66, p<0.01)$. Therefore, we report a negative 
relationship between Affective empathy and IA (most influenced by the PD scale, since EC exhibited no significant relationship).

Relationship between Cognitive Empathy and Interoceptive Awareness

We observed a positive relationship between Cognitive Empathy and the Awareness of Mind Body Integration subscale, $r(26)=0.35, p=0.06$, although it did not survive multiple comparison correction. In addition, we observed a significant positive relationship between the IRI Perspective Taking (PT) subscale and the MAIA Awareness of Mind Body Integration subscale, $r(26)=$ $0.41, p<0.01$. Therefore, we report a positive relationship between Cognitive empathy and IA (most influenced by the PT subscale, since Fantasy exhibited no significant relationship).

Taken together, our behavioral results show a bidirectional relationship between empathy and IA, depending on the facet of empathy interrogated and mainly driven by the subscales of PD (Affective Empathy) and PT (Cognitive Empathy).

Functional Connectivity Results

Network Connectivity 
We observed that within a network comprising right inferior frontal operculum (rIFO), bilateral superior parietal lobule (SPL) and bilateral middle temporal gyrus (MTG), greater connectivity in the rIFO was associated with greater overall empathy as measured by the IRI Total, and with greater IRI Affective empathy. Conversely, lower connectivity in the rIFO was associated with increased overall IA as measured by the MAIA Total and increased MAIA Capacity to Regulate Attention (Figure 13, Table 2).

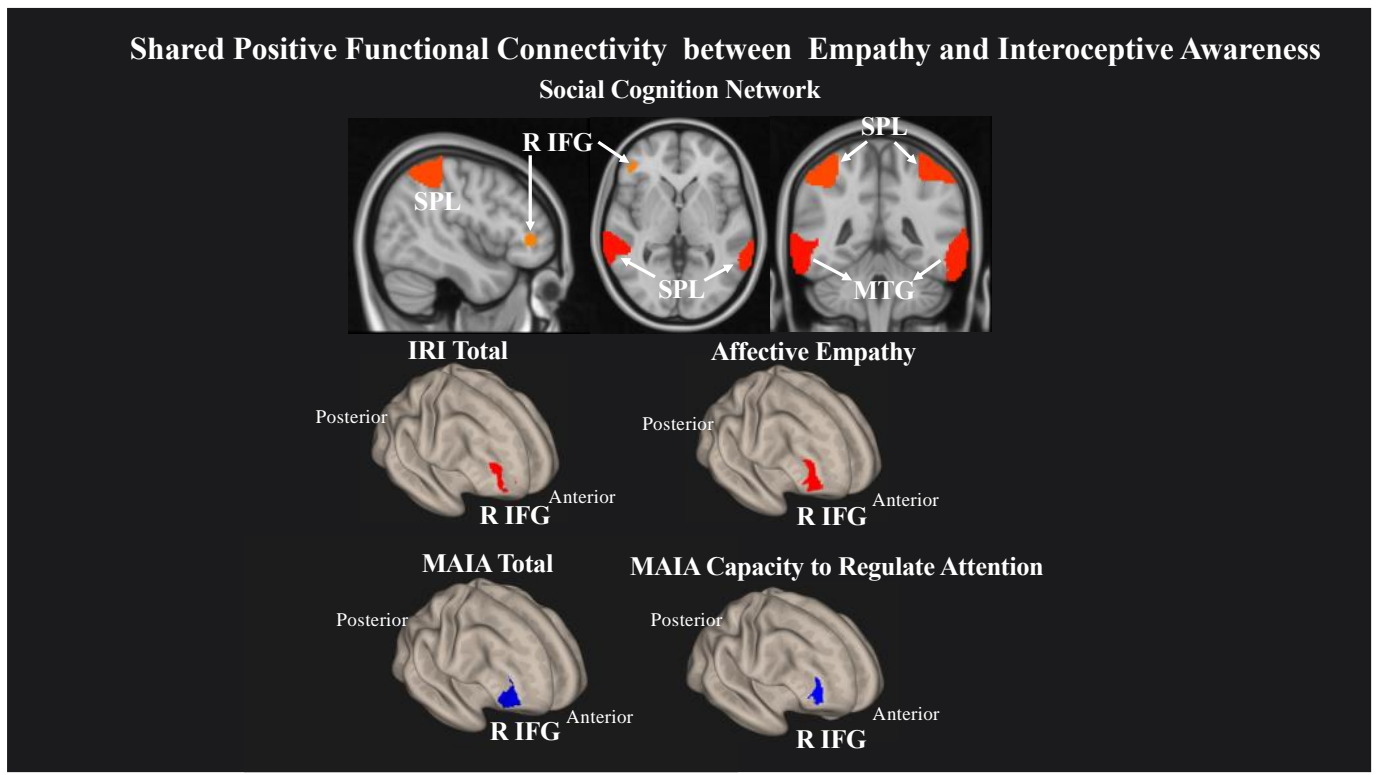

Figure 13. Greater connectivity in the right inferior frontal operculum (rIFO) is associated with lower total IA and greater Affective empathy. Statistical maps are FDR-corrected within the network at cluster-based $p<0.05$, after voxel threshold at $p<0.001$, and further corrected for age, gender, and multiple comparisons across components using FDR.

\section{Table 2}




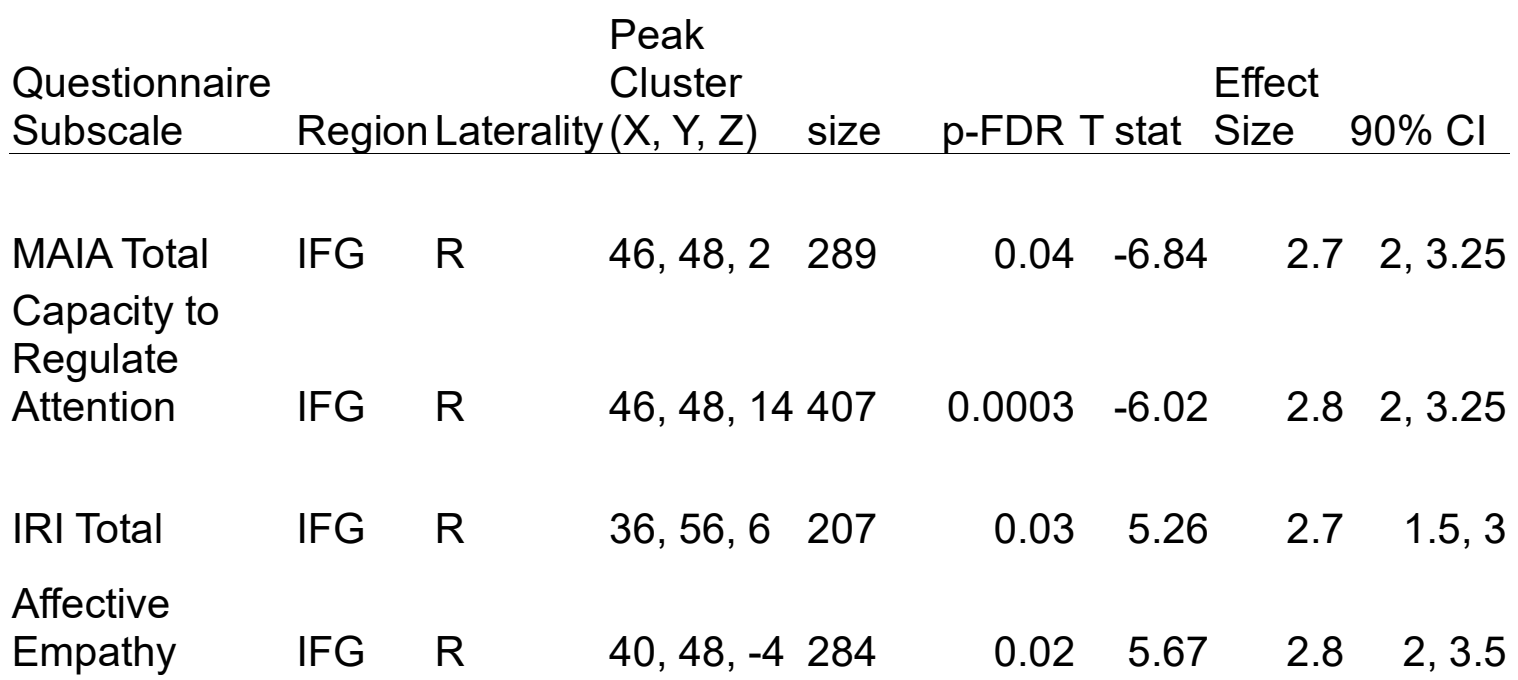

Network Variability

Network variability analyses revealed that within a network comprising left IFO (L IFO), Cerebellum, and rAI, IRI Personal Distress was negatively related to variability of the network $(T(22)=-1.04, p=0.02))$, while conversely, MAIA 
Awareness of Body Sensations was positively related $(T(22)=1.48, p=0.001))$,

(Figure 14).

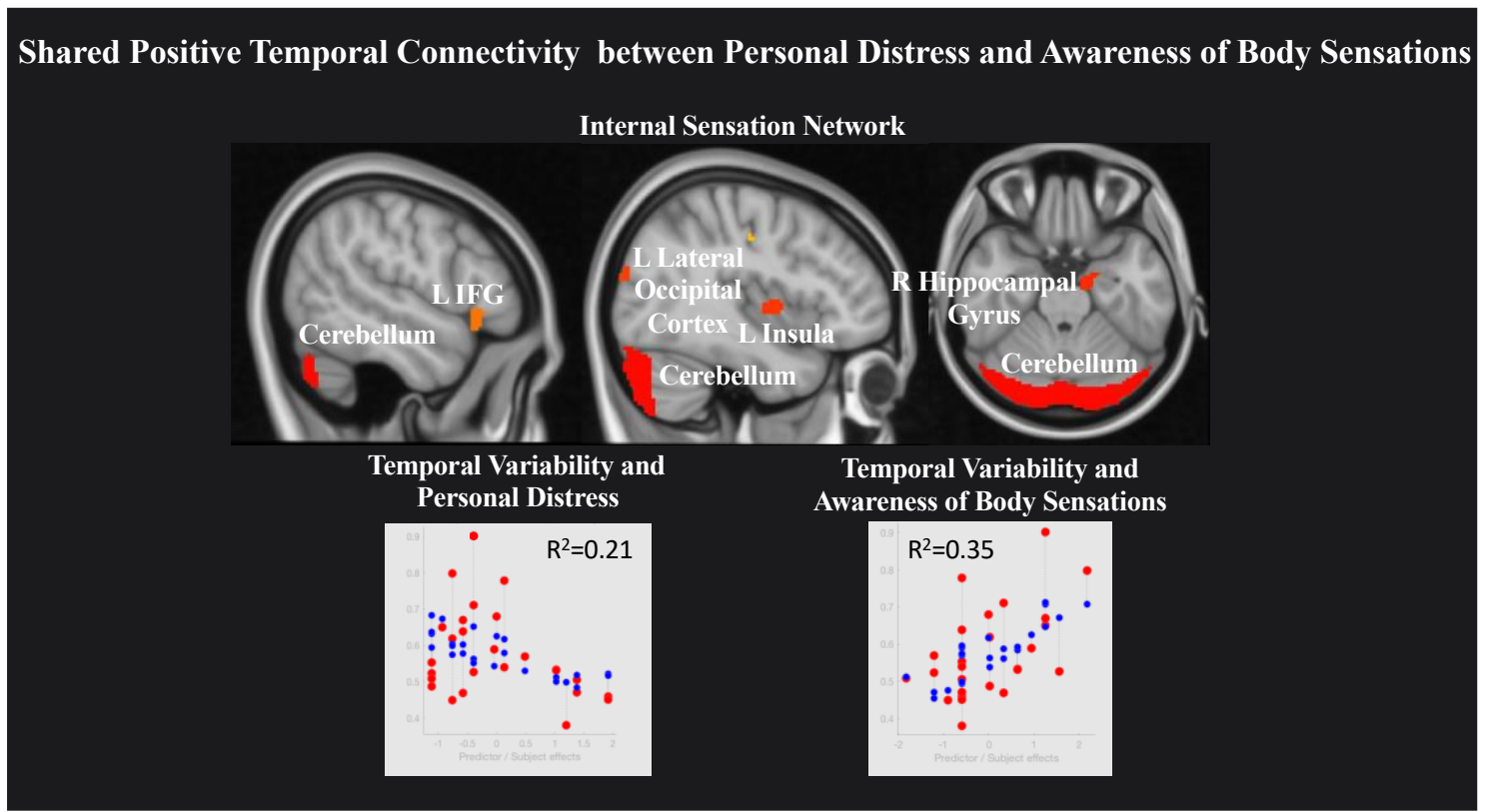

Figure 14: Shared network variability between Affective empathy and MAIA Awareness of Body Sensations. Statistical maps are FDR-corrected within the network at cluster-based $p<0.05$, after voxel threshold at $p<0.001$, and further corrected for age, gender, and multiple comparisons across components using FDR. Note: the red dots in the graph represent the observed correlation between the standard deviation of the individual network's BOLD time-series (SDBOLD) and each subjects' behavioral measure. The blue dots represent the predicted values of the statistical model. The $R^{2}$ value represents the variance explained resulting from the regression between SDвoLD and behavioral variables of interest. 
Lastly, within a network comprising right Precuneus, rMTG, bilateral supramarginal gyrus (SMG) and rIFO, IRI Perspective Taking $(T(22)=1.45$, $\mathrm{p}=0.001)$ and MAIA Mind Body Integration $(T(22)=2.52, \mathrm{p}=0.01)$ were positively related to network variability (Figure 15).

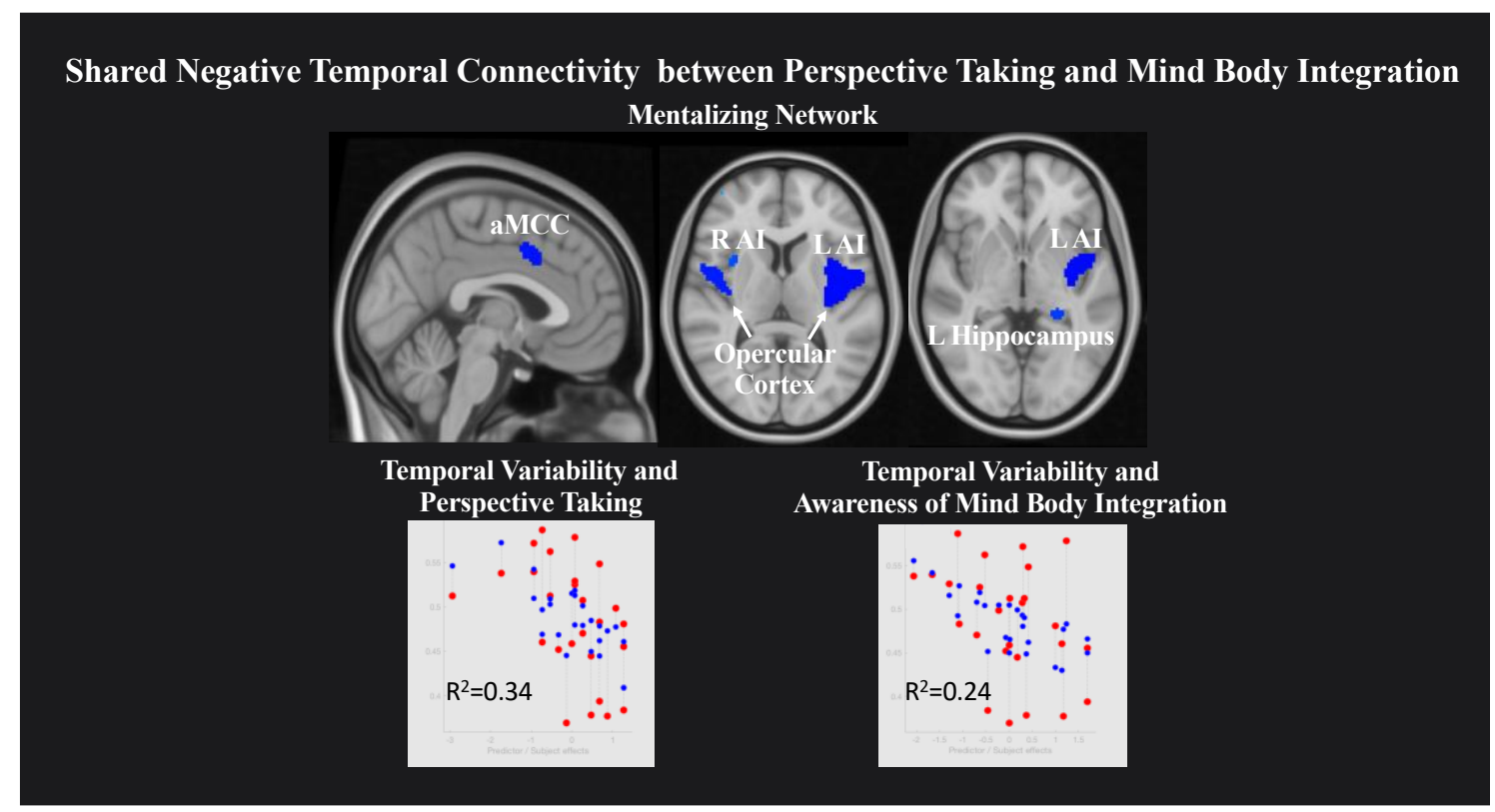

Figure 15: Shared network variability between Cognitive empathy and MAIA Mind-Body Integration statistical maps are FDR-corrected within the network at cluster-based $p<0.05$, after the voxel threshold at $p<0.001$, and further corrected for age, gender, and multiple comparisons across components using FDR. Note: the red dots in the graph represent the observed correlation between the standard deviation of the individual network's BOLD time-series (SDBOLD) and each subjects' behavioral measure. The blue dots represent the predicted values of the statistical model. The $R^{2}$ value represents the variance explained resulting from the regression between $S D_{\text {воLD }}$ and behavioral variables of interest.

\section{Discussion}

Empathy and IA are crucial to meaningful social exchanges. As these two constructs interact, a 'shared representation' is created as one's own internal state is utilized to understand the emotional experiences of others (Decety \& 
Sommerville, 2003). However, it is not yet clear whether a specific aspect of empathy (Affective or Cognitive) interfaces with IA. Our resting state fMRI study employed Independent Component Analysis (ICA) to investigate which empathy facet shares rsFC and/or rsBOLD with IA while healthy adults viewed naturalistic stimuli. We observed a bidirectional behavioral relationship between empathy and IA, whereby Affective empathy and IA were negatively related, and Cognitive empathy and IA were positively related. This bidirectional link is mirrored in the neuroimaging results, such that Affective empathy and IA were inversely related to increased rsFC within the rIFO, and also inversely related to rsBOLD; whereas Cognitive empathy and IA showed only a positive relationship with rSBOLD. Together, these results suggest a double disassociation between empathy and IA depending on the type of empathy interrogated, which is reflected in the brain network's intrinsic connectivity and variability patterns.

\section{Behavioral Findings}

Behaviorally, we observed a negative relationship between the Personal Distress (PD) subscale of the Affective empathy aggregate IRI scale and the total MAIA score, Capacity to Regulate Attention and Trusting Body Sensations subscales. The Capacity to Regulate Attention subscale pertains to various ways of controlling one's attention towards bodily sensations, as part of an active regulatory process; while Trusting Body Sensations reflects the extent to which one views awareness of bodily sensations as helpful for decision making (Mehling et al., 2012). The Empathic Concern (EC) subscale of Affective 
empathy exhibited no significant relationship, implying that PD is the dominant subscale of the Affective empathy aggregate scale when relating to the MAIA within this sample. This distinction is important, considering that previous data suggests EC motivates individuals to pay attention to others' emotions in order to try to comfort them, while conversely, PD drives attention away from others in order to reduce the aversive effects for oneself, perhaps as a form of emotion regulation (Zaki, 2014). Indeed, Decety \& Jackson, 2004 proposed that PD may arise from the failure of applying sufficient self-regulatory control over the shared emotional state. In line with previous studies, we report an inverse relationship between PD and an attention regulation measure - MAIA's Capacity to Regulate Attention subscale. Together with the Trusting Body Sensations subscale, our findings suggest the increased ability to regulate internal attention and rely on this discrete information may be linked to a decrease in the discomfort experienced while witnessing another's distress.

Furthermore, we found a positive relationship between the Perspective Taking (PT) subscale of the Cognitive empathy aggregate IRI scale and the Awareness of Mind Body Integration of the MAIA. This MAIA subscale represents the integration of several higher level cognitive processes necessary for socially relevant goal-directed behavior (i.e. executive functions (PRIBRAM, 1973)) including: emotional awareness, self-regulation of emotions, and the ability to feel a sense of an embodied self, that is - "a sense of the interconnectedness of mental, emotional, and physical processes as opposed to a disembodied sense 
of alienation from one's body" (Mehling et al., 2012). Thus, our results support previous findings suggesting Cognitive empathy, and in particular PT, is related to a wide array of executive function skills such as working memory, inhibitory control and cognitive flexibility (Aliakbari et al., 2013; Healey \& Grossman, 2018; Yan et al., 2020). Taken together with the aforementioned negative relationship between PD and IA, these behavioral results suggest a bidirectional 'shared representation' between empathy and IA, contingent on the type of empathy interrogated. To wit, directing attention towards internal bodily sensations may relieve vicarious emotional pain but flexibly employing cognitive-control skills may increase the ability to take the perspective of another.

\section{Functional Connectivity Findings}

Our rsFC results provide further support for this inverse relationship. Within a network of brain regions previously shown to underlie attentional processing (superior parietal lobule (SPL), medial temporal gyrus (MTG) and right inferior frontal operculum (rIFO) (Corbetta \& Shulman, 2002; Perrett et al., 1992, 1982, 1985; Yan Wu, Wang, et al., 2016), we observed that increased rsFC in the rIFO was associated with increased overall empathy (total IRI score) and the Affective empathy aggregate scale on one hand, but reduced overall IA (total MAIA score) and Capacity to Regulate Attention on the other hand. Previous studies investigating both personal (Craig, 2009; Critchley, 2005; Damasio, 2005; Gray et al., 2007; Johnson, 2001) and vicarious emotional experience (Jabbi et al., 2007; Singer et al., 2004) show the consistent activation 
of the anterior insula (AI) and frontal operculum, and therefore the IFO is thought of as a continuum between these two structures (Jabbi et al., 2007; Wicker et al., 2003). Because Affective empathy was driven by the PD subscale within this sample, increased rsFC within the rIFO in the present study may relate to intensified personal suffering from witnessing another's distress, but decreased awareness of one's own body sensations, perhaps due to allocating attention externally (for example, away from self and toward other's distress). In line with previous activation-based results (Adolfi et al., 2017; Ernst et al., 2013), our findings refine the 'shared representation' hypothesis (Decety \& Sommerville, 2003) by showing rsFC overlap of IA and Affective empathy in this region, and extend previous results by providing rsFC evidence of a double dissociation between empathy and IA.

Network Variability Findings

Our rsBOLD results offer a complementary perspective that further supports this bidirectional relationship. We observed increased scores on the MAIA Awareness of Body Sensations subscale and decreased scores on the Affective empathy scale was associated with increased rSBOLD of brain regions previously shown to underlie processing and integration of visceral information (i.e., Cerebellum, L IFO, L Al) (Adamaszek et al., 2017; Baumann \& Mattingley, 2012; Bogg \& Lasecki, 2015; Schienle \& Scharmüller, 2013; J. D. Schmahmann, 2001; Terasawa et al., 2013). Despite the prevailing focus on the Al as a hub for processing body sensations (Kuehn et al., 2016; Pollatos et al., 2007; Singer et 
al., 2009; Terasawa et al., 2013), additional brain regions are also commonly implicated in interoceptive experience. For example, fMRI studies identify the involvement of the IFO and cerebellum, reinforcing the notion that interoceptive processing (and perhaps especially nociceptive information) may occur through multiple neural pathways (Garcia-Larrea, 2012; Peiffer et al., 2008; Rapps et al., 2008). In the same vein, observing distress in others without actually experiencing it may rely on high-order cognitive functions to access minor changes in physical state, as a tool to modulate negative stimulus input (Preckel et al., 2018). The implication of the cerebellum in a shared network underlying both Affective empathy and interoceptive processing is not surprising, since the cerebellum serves as an integral node in the distributed cortical-subcortical neural circuitry supporting an array of sociocognitive operations (Jeremy D. Schmahmann \& Pandya, 1995). Thus, our rsBOLD findings offer a complementary perspective alongside our rsFC data, and suggest that increased communication between regions of this network relates to increased awareness of internal sensations and perhaps a sense of 'self', but decreased flexibility in integrating emotions arising from witnessing 'others" distress.

In addition, we observed a positive relationship between rSBOLD and the Cognitive empathy scale and the MAIA Awareness of Mind Body Integration subscale within a network of brain regions previously associated with the process of mentalizing - the precuneus, rIFO, SMG and MTG (Mar, 2011; Northoff et al., 2006; Schurz et al., 2014; Spreng, Mar, et al., 2009; Vogeley et al., 2001). 
Mentalizing signifies the ability to attribute mental states to another individual, allowing the observer to predict intent and direct their behavior appropriately (U. Frith et al., 1991; Premack \& Woodruff, 1978). Researchers agree that mentalizing differs from the vicarious sharing of emotion in its psychological complexity, combining observation, memory, knowledge, and reasoning to provide insight into the thoughts and feelings of others (Decety \& Jackson, 2004; van der Heiden et al., 2013). Therefore, its connection with MAIA's Awareness of Mind Body Integration scale is not surprising, since both concepts require not only affective experience, but also comprehension and integration of another's particular state of mind within one's own emotional schema. We therefore suggest that increased rsBOLD of brain regions underlying a mentalizing network may point to enhanced network flexibility subserving not only a better ability to take another's perspective, but also an improved sense of interconnectedness between one's own mind and body.

Lastly, our data shows an interesting convergence of empathy and IA within the IFO. Research suggests the IFO serves as both a sensory-cognitive integration area and a control node of the ventral attention network (Corbetta \& Shulman, 2002; Craig, 2009), conjectured to maintain goal-related information online until a decision is reached (Tops et al., 2011; Tops \& De Jong, 2006). Moreover, recent evidence suggests a hemispheric specialization of the IFO related to reactive/proactive goal maintenance (Tops et al., 2011). On one hand, the rIFO may facilitate immediate somatosensory processing and attentional 
shifting whilst a response is ongoing (reactive) (Hampshire et al., 2009; Higo et al., 2011; Nelson et al., 2010; Tops et al., 2011), through its connections to rostral ACC, superior temporal gyrus (STG) and occipital cortex (Cauda et al., 2011). On the other hand, the IIFO may exert top-down control whilst preparing a response (proactive) (Tops et al., 2011), through its connections to dorsolateral prefrontal cortex and bilateral supplementary motor area (Cauda et al., 2011). Taking this evidence into consideration, we speculate the positive association between rsFC within the rIFO and Affective empathy indicates a propensity in the highly empathic individual to shift attention toward salient cues in their environment (for example, another individual in distress). In contrast, the negative relationship between rsFC within the rIFO and IA may indicate an inability to redirect attention toward external salient cues, and therefore may lead to increased awareness of one's own internal sensations. Our rsBOLD findings offer complementary evidence regarding the role of the IFO in socioemotional processes. We show that increased network flexibility within an interoceptive experience network (comprised of IIFO, L Al, cerebellum) is linked to increased Awareness of Body Sensations as well as decreased Affective empathy. We suggest the IIFO plays a crucial part in this network's ability to modulate attention from one's own internal sensations (i.e. the 'self') to discomfort arising from witnessing the 'others" distress, perhaps in an effort to plan an appropriate emotional response. In the same vein, we show enhanced network flexibility within a mentalizing network (comprised of rIFO, precuneus, SMG, MTG) is 
related to both better Cognitive empathy and increased Mind Body Integration. These relationships may illustrate that heightened ability to determine intent in others and integrate sensory information into one's own emotional schema relates to flexibly shifting attention towards the target of interest (either 'self' or 'other'). In sum, our data suggests the IFO may serve as an internal/external attention modulator and thus may play a critical role in switching attention from one's own body sensations ('self'; IA) to the other's (Affective and/or Cognitive empathy).

\section{Limitations and Future Directions}

Our study's findings should be considered along with its limitations. The definition of rsBOLD has been inconsistent across previous studies, (e.g., amplitude, variance, standard deviation, mean squared successive difference; for a review, see (Garrett, Samanez-Larkin, et al., 2013) with considerable range in the methodology used to derive them. Therefore, implementation of rsBOLD as a consistently used neuroimaging measure will require increased efforts toward methodological standardization. It is also important to note that due to the nature of the analyses used, the findings of this study do not represent causal relationships. That is, the results represent a correlational relationship between a questionnaire-based measure of IA or empathy and rsFC and/or rsBOLD. Our sample was unfortunately not large enough for a gender-specific analysis, as evidence suggests there are differences in the capacity for empathy between males and females (Christov-Moore et al., 2014). Future research should be 
conducted in this regard. Similarly, in using an undergraduate sample, the generalizability of these findings is limited.

\section{Summary}

In conclusion, the current research provides novel information about the relationship between interoceptive awareness and empathy. In contrast to previous studies which used task-based fMRI to assess the neurobiology of these two constructs separately, we used a data-driven resting state approach to test whether distinct empathy facets share network characteristics (rsFC/rsBOLD) with IA. We demonstrate a bidirectional relationship between empathy and IA, depending on the type of empathy investigated. Specifically, Affective empathy and IA share rsFC and rsBOLD, while Cognitive empathy and IA only share rsBOLD. In regard to Affective empathy, increased vicarious emotional experience and decreased IA were associated with increased rsFC within the rIFO of a larger attention network; while increased IA and reduced Affective Empathy were related to increased network flexibility within an internal sensation network. Concerning Cognitive empathy, perspective-taking ability and a sense of mind-body connectedness related to increased communication between brain regions subserving a mentalizing network. We also suggest the role of the IFO as an internal/external attention modulator that may play a critical role in switching attention from one's own body sensations (IA) to another's (Affective and/or Cognitive empathy). Overall, we show that the ability to feel and 
understand another's emotional state is related to one's own awareness of internal body changes, and that this relationship is reflected in the brain's intrinsic neuroarchitecture. Methodologically, this work highlights the importance of utilizing rsBOLD alongside rsFC as an important complementary route into understanding neurological phenomena. Our results hold promise in aiding diagnosis of clinical disorders characterized by IA and empathy deficits such as the autism spectrum disorders (ASD), where participants may be unable to complete tasks or questionnaires due to the severity of their symptoms.

\section{EXPERIMENT 3:}

Convergent Neural Correlates of Empathy and Anxiety During Socioemotional Processing

Empathy is characterized by the ability to understand and share an emotional experience with another person. This socioemotional response induced by perceiving another person's affective state is a fundamental component of social interactions and is thought to aid in both moral development and prosocial behavior (Decety et al., 2015). Most literature suggests that empathy encompasses two interrelated components: affective empathy and cognitive empathy (Davis, 1983). Affective empathy, also termed empathetic arousal, is the automatic process of vicariously experiencing the emotional state 
of another person (I feel what you feel). In comparison, cognitive empathy is related to perspective-taking, or the ability to adopt another's psychological pointof-view, also referred to as mentalizing (I understand what you feel). While affective empathy is believed to be more innate, fostering care and concern for others, cognitive empathy involves a deliberate understanding of another person's viewpoint and is particularly important for social competence and reasoning (Decety et al., 2015) as the inability to understand another person's beliefs and actions may interfere with appropriate social responses (Ickes \& Hodges, 2013; Simone G. Shamay-Tsoory et al., 2007).

Given that empathy is closely tied to compassion and concern for others, the increased emotional awareness and social sensitivity in empathy may consequently be associated with anxiety, particularly when empathizing with individuals in distressing situations. Conversely, increased anxiety may translate into increased worry and concern for others, or concern for how one's actions might affect others, and therefore may be linked to increased empathy. Gaining insight from clinical anxiety, individuals with social anxiety disorder tend to be hyper self-aware and extremely attentive to social signals due to their intense fear of being negatively evaluated by peers (Perry et al., 2011). This understanding may help explain why anxious individuals have a propensity to over-interpret the implied threat in others' facial expressions (Horley et al., 2004), but moreover, these internal and external attentional biases found in anxiety may also be related to enhanced socioemotional processing abilities (Perry et al., 
2011). Indeed, it has been suggested that increased sensitivity to subtle social cues is essential to make mentalizing judgments (Harkness et al., 2005).

Beyond commonalities of enhanced emotional and social sensitivity, empathy and anxiety may additionally be related through increased internally generated thought. Research indicates that enhanced self-reflection is positively correlated with perspective-taking and empathic concern (Joireman et al., 2002) and may even enhance sensitivity leading to more accurate judgments about other's mental states (Dimaggio et al., 2008). Building on this notion, neuroimaging research provides additional evidence that when predicting emotional responses of another person, greater recruitment of emotion related and mentalizing regions positively correlates with self-report empathy (Hooker et al., 2008). Similarly, internally generated thought is believed to be a key constituent in anxiety. Meta-analyses show associations between rumination and anxiety, with the strongest links being attributed to brooding and emotion-driven rumination (Olatunji et al., 2013). In fact, rumination has been shown to mediate the longitudinal relationship between life stress and symptoms of anxiety in both adolescents and adults (McLaughlin \& Nolen-Hoeksema, 2011). Neuroimaging investigations reveal that rumination reliably engages the cortical midline, especially the more anterior portion, such that increased engagement of the medial prefrontal cortex (MPFC) was found when healthy subjects were instructed to adopt a ruminative thinking style in an autobiographical memory task (Kross et al., 2009; Nejad et al., 2013). Aberrant 
and sustained recruitment of the amygdala has also been implicated in rumination. During one self-referential processing task in depressed patients, the amygdala was found to exhibit more sustained responses to emotional relative to neutral stimuli, with degree of sustained amygdala activation being positively correlated with rumination scores (Siegle et al., 2002).Together, this suggests that empathy and anxiety may be linked not only through a sensitivity to social and emotional information, but also in a shared propensity to continue to process emotional information through reflection and rumination.

Although the literature is scant, previous research provides some evidence for a direct relation between empathy and anxiety. Using film clips depicting victims facing threats, one recent study demonstrated that trait empathy is associated with greater self-reported vicarious anxiety when observing victims, and a follow-up study extended these findings by manipulating levels of state empathy to establish a causal relationship between empathy and vicarious anxiety (Shu et al., 2017). Furthermore, previous studies have documented that individuals who experience emotion mirroring intensely are more prone to personal distress (e.g., anxiety or discomfort; (Lamm et al., 2007)). Investigations in the clinical realm have reported similar relationships. A recent study on inpatient adolescents found that measures of affective empathy are positively related to all anxiety dimensions (Gambin \& Sharp, 2018). Likewise, Perry et al., 2011 found that individuals with social anxiety disorder demonstrate unique 
socio-cognitive abilities, such that measures of social anxiety and general anxiety positively associated with affective and cognitive empathy, respectively.

To date, neuroimaging studies have only separately investigated empathy and anxiety. In regard to empathy, affective and cognitive empathy involve interacting and partially overlapping neural bases (Y. Fan et al., 2011). Because affective empathy has been linked to automaticity relative to its cognitive component, it primarily elicits activations from regions implicated in rapid and prioritized processing of emotion signals, including: the amygdala, hypothalamus, orbitofrontal cortex (OFC) and anterior insula (Decety et al., 2013). By comparison, cognitive empathy, which shares similar neural networks with perspective taking and mentalizing (Pardini \& Nichelli, 2009), additionally involves the superior temporal sulcus (STS), temporoparietal junction (TPJ), fusiform gyms (FG), and (mPFC; (Saxe \& Powell, 2006)). The involvement of STS and TPJ, areas posited to contain mirror neurons (Iriki, 2006), suggests these areas are specifically activated during the conscious determination of intent. Lesion studies and recent clinical work support the involvement of aforementioned regions in relation to empathic responses. In patients with frontotemporal dementia, reduction of gray matter in the amygdala, insula and TPJ were associated with deficits in attribution of mental states (i.e., mentalizing), specifically in the emotional realm (Cerami et al., 2014). Furthermore, lesions in the amygdala and insula have been found to specifically be associated with deficits in affective empathy (Leigh et al., 2013), while patients with mPFC 
damage show consistent and selective deficits in cognitive empathy but intact emotion recognition and affective empathy (S G Shamay-Tsoory et al., 2003). For the purposes of this study, however, we did not aim to further disentangle the neural differences between cognitive and affective empathy, but instead applied this knowledge to more precisely investigate the links between empathy and anxiety.

In regard to anxiety, neuroimaging investigations demonstrate that anxiety reliably elicits activations in the amygdala and insula, particularly in relation to negative emotional responses (Etkin \& Wager, 2007). While the amygdala is central to threat detection, orchestrating behavioral and physiological responses, the insula has been implicated in interoceptive awareness and may be particularly important for sensing perturbations in social anxiety disorder, PTSD and specific phobia consistently show greater activations than matched control subjects in these structures, however, similar patterns have been observed during fear conditioning in healthy subjects (Etkin \& Wager, 2007). Extensive work additionally highlights the role of the hippocampus in anxiety due to its importance in contextual processing, as well as the mPFC, which provides topdown regulatory control to the amygdala, helping to modulate behavior in light of complex environmental information (Liberzon et al., 2015; Shin \& Liberzon, 2009). Interestingly, many of the regions involved in anxiety are also implicated in empathy, namely the amygdala, insula and mPFC. This evidence suggests empathy and anxiety may modulate processing in similar underlying neural 
networks, as both constructs are associated with regions involved in processing salient stimuli, subjective emotional experience, and understanding complex social interactions.

\section{Aims}

Therefore, given evidence of a relation between empathy and anxiety, the current study aimed to investigate the underlying convergent neural correlates using a socially relevant task shown to engage cognitive and emotion processes through face processing, introspection and self-report rating of emotional state. This task was then used as a backdrop to explore how differences in trait empathy and anxiety may modulate processing within a socioemotional processing network and to assess points of convergence between empathy and anxiety.

\section{Hypotheses}

The following hypotheses were posited: (1) behaviorally, higher empathy would be related to higher trait anxiety; (2) increased trait anxiety and empathy would share neural substrates in emotional processing structures (e.g., amygdala and insula); while empathy will additionally relate to regions supporting social cognition (e.g., TPJ); and (3) both empathy and anxiety would exhibit increased functional connectivity (FC) between regions supporting emotional processing and social cognition (e.g., amygdala to TPJ), thus demonstrating the two constructs are linked within a socioemotional network.

Methods 


\section{Participants}

55 healthy young adults were recruited for the present study. All participants were right-handed, with normal or corrected-to-normal vision and hearing and had no disclosed history of neurological or psychiatric disorders. A total of six participants were excluded from analyses due to incomplete behavioral data $(\mathrm{N}=1)$, incomplete magnetic resonance imaging $(\mathrm{MRI})$ data $(\mathrm{N}=$ 4), and disclosed psychiatric diagnosis following consent $(N=1)$, leaving 49 participants in the final sample (28 males, 21 females; Ages 18-33; $\mathrm{M}$ age = $22.00, \mathrm{SD}=4.04)$

\section{Procedure}

The present study consisted of multiple visits. On the first visit, participants completed the functional MRI (fMRI) portion of the study at the University of Louisville, School of Medicine. At this time, participants signed consent forms, were briefed on MRI protocol, read through task instructions and completed the Face Processing Task. Within the next $72 \mathrm{~h}$, participants visited the laboratory on the main campus to complete a variety of self-report questionnaires measuring personality traits of empathy and anxiety, along with measurements assessing internally generated thought (i.e., worry and rumination): Toronto Empathy Questionnaire (TEQ;(Spreng, McKinnon, et al., 2009)), State-Trait Anxiety Inventory (STAI;(Spielberger et al., 1973)), Penn State Worry Questionnaire (PSWQ;(Meyer et al., 1990)), and Ruminative Responses Scale (RRS; (Treynor, 2003)). 
The TEQ was chosen as the measure for empathy because it possesses a robust single factor structure and is thought to measure empathy at the broadest level (i.e., capturing both affective and cognitive empathy), and has convergent validity with existing self-report scales and behavioral measures of affective and cognitive empathy (Spreng, Mar, et al., 2009). The RRS consists of 22 items, comprising three subscales: (1) reflection-turning inward to engage in cognitive problem solving; (2) brooding-comparing one's current situation with some unachieved standard; and (3) depressive Rumination. Subscale totals of the RRS can be individually utilized, or all items can be summed together for a composite total rumination score, indicative of one's propensity to engage in repetitive and passive self-focused attention (Treynor, 2003). Since all questionnaires were administered one to 3 days following fMRI task scanning, only trait anxiety scores from the STAI were used in analyses. All questionnaires are known to be psychometrically sound, with high internal consistency and testretest reliability (Barnes et al., 2002; Meyer et al., 1990; Spreng, Mar, et al., 2009; Treynor, 2003).

fMRI Task Design 
A Face Processing Task constructed from Fearful and Neutral human faces (male and female) shown to reliably elicit activation from brain regions in a canonical socioemotional network was utilized. The Face Processing task consisted of two conditions: Fearful and Neutral. Images were acquired from the Eckman Face Database. During scanning, visual stimuli were displayed through ePrime onto an in vivo Esys LCD TV monitor at the back of the scanner bore, which was viewed by participants through a mirror on the head-coil. This task employed an event-related design, during which face stimuli were presented for $4 \mathrm{~s}$ in a pseudo-random order (Fear trials $\mathrm{N}=30$, Neutral trials $\mathrm{N}=12$ ). After presentation of each face, participants rated the image for how negative it made them feel, using a four-point Likert scale ( 1 = None, 4 = Extremely negative; $2 \mathrm{~s}$ to rate). Each trial was then followed by a variable inter-trial interval (ITI; 0-8 s). This rating period was included to ensure processing of the emotionality of each face and encourage introspection (Figure 16).

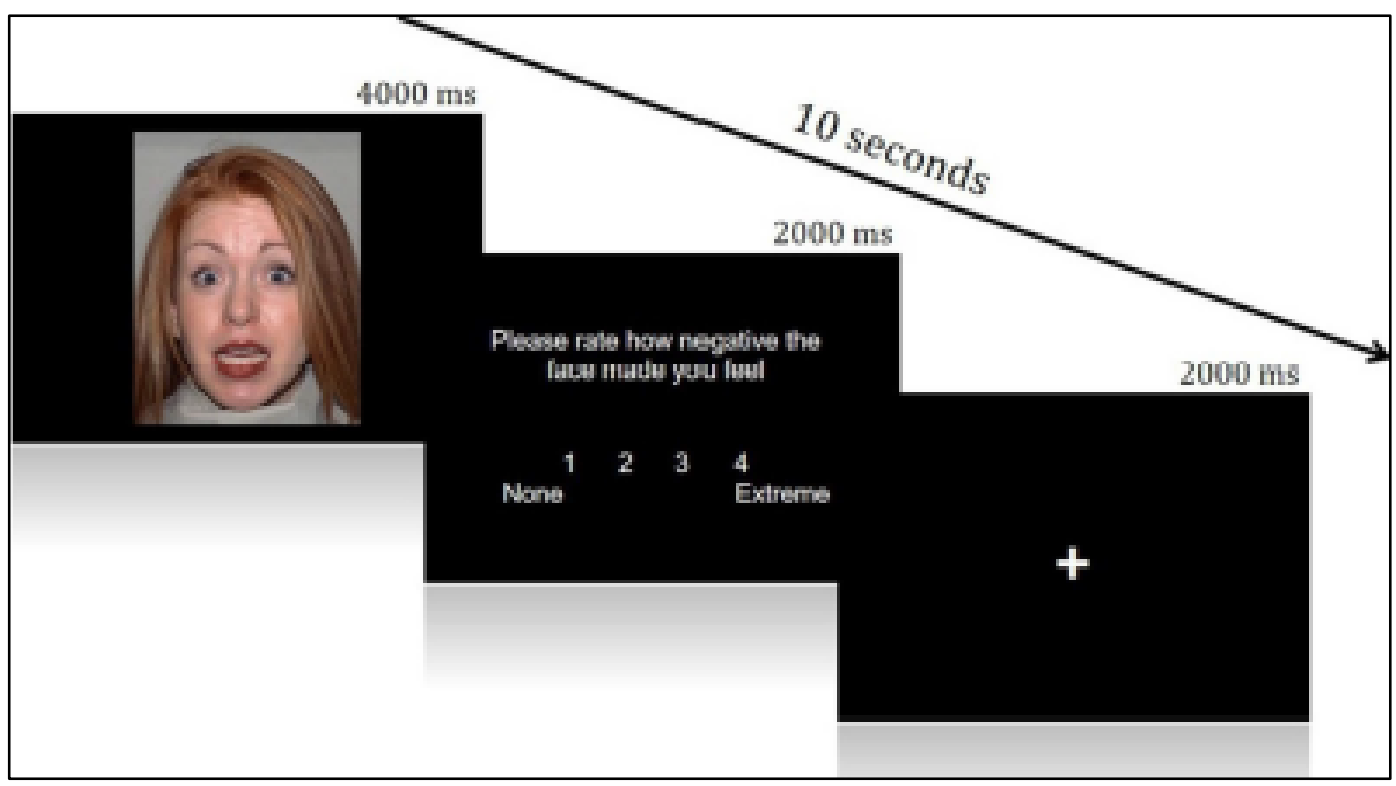


Figure 16: Example of a single trial from the Face Processing Task.

Behavioral data were analyzed using SPSS (Version 25.0.0; SPSS, INC.), including the use of PROCESS for mediation analyses (Hayes, 2012). Here, we chose not to use a causal steps approach to mediation, as this method has been criticized as being unnecessarily restrictive and can lead to misleading or false conclusions (e.g., concealing a significant indirect effect due to the absence of a total or direct effect, i.e., suppression;(MacKinnon et al., 2000; Rucker et al., 2011)). Furthermore, we did not seek to demonstrate causal effects, but rather describe relationships among traits. For these reasons, a bootstrapping method was undertaken to test for indirect effects (Preacher \& Hayes, 2004).

Neuroimaging Analysis

Functional Analyses

Image preprocessing and data analysis were implemented using the FSL package (version 5.0.9, Analysis group, FMRIB, Oxford, UK). Standard preprocessing was applied: MCFLIRT-linear slice-time correction/motion correction, optiBET-brain extraction (Lutkenhoff et al., 2014), time-series prewhitening, and high-pass filtering $(0.01 \mathrm{~Hz})$. Individual's functional images were first registered to their high-resolution MPRAGE scans via 6-parameter linear registration, and the MPRAGE images were in turn registered to the Montreal Neurological Institute (MNI) 152 TI-2 mm template via a 12-parameter nonlinear registration (Andersson et al., 2008). These registrations were 
combined to align the functional images to the standard template. Functional images were resampled into the standard space with $2 \mathrm{~mm}$ isotropic voxels and were smoothed with a Gaussian kernel of $6 \mathrm{~mm}$ full-width at half-maximum (FWHM). ICA-AROMA (i.e., Independent Component Analysis Automatic Removal of Motion Artifact), a data-driven method to identify and remove components representing motion-related noise in $\mathrm{fMRI}$ data, was additionally utilized (Pruim et al., 2015). Following preprocessing, Lower-level statistics were implemented in FEAT. Using multiple regression analysis, statistical maps representing the association between the observed time-series (e.g., BOLD signal) and one or a linear combination of regressors for each subject were constructed. Regressors for the main effects were constructed by modeling each of the conditions-Fearful and Neutral-versus low-level fMRI baseline (ITI fixation), in order to create contrasts of interest: Fearful $>$ Neutral $(F>N)$ and Neutral $>$ Fearful $(\mathrm{N}>\mathrm{F})$. For each regressor, a double-gamma hemodynamic response function (HRF) was convolved with an event vector starting at the stimulus onset through rating response to capture both the stimulus processing and introspective time periods in each trial (duration of 6,000 ms). Higher-level analysis was conducted using FLAME $1+2$ and outlier de-weighting to combine and spatially normalize all subjects. The higher-level models employed nonparametric permutation methods through FSL's randomize function (Nichols \& Holmes, 2001). For each contrast of interest, cluster thresholding and correction for multiple comparisons were implemented through the Threshold- 
Free Cluster Enhancement (TFCE) method, which detects clusters of contiguous voxels without first setting an arbitrary statistical cutoff (e.g., $Z>2.58$ ), and controls the family-wise error (FWE) rate at $\mathrm{p}<0.05$ (Smith \& Nichols, 2009). Each contrast underwent 5,000 permutations. Randomise produces corrected 1p maps, which were used for all figures. Figures of statistical brain maps were created using FSLview.

The present analyses primarily focused on a region of interest $(\mathrm{ROI})$ approach. For ROI analyses, regions comprising a canonical socioemotional processing network were analyzed (i.e., regions supporting emotion processing and social cognition). ROls included: bilateral TPJ, bilateral insula, mPFC, and bilateral amygdala. Convergent findings from neuroimaging and lesion studies indicate that the amygdala and insula are critical for affective reactivity and interoceptive awareness, respectively (Craig, 2002; Etkin \& Wager, 2007), while the TPJ and mPFC are essential to the perception of intentionality and mental states of others, as well as cognitive empathy (Amodio \& Frith, 2006; Young et al., 2010). Bilateral anatomical masks were created from FSL's Harvard Oxford cortical atlas (insula and mPFC) and subcortical atlas (amygdala). Since this cortical atlas does not include anatomical TPJ masks, bilateral masks were manually created, using $10 \mathrm{~mm}$ radii surrounding the vertices at which the temporal and parietal lobes meet, at the posterior end of the Sylvian fissure for each hemisphere. The STS was additionally tested $(-60,-46,6)$ but yielded null results after correcting for multiple comparisons and is thus not discussed further. 
Secondly, exploratory whole-brain analyses were carried out for the Face Processing Task.

Functional Connectivity

Seed regions for FC analyses were derived from peak z-stats of functional activation task results (peak z-stat within FWE-corrected cluster, when applicable, or peak z-stat within ROI mask in cases when no significant functional activation results were found). Seed masks were created using a $5 \mathrm{~mm}$ radius surrounding the peak z-stat. Seed-to-ROI FC was then performed by using the following steps. First, lower-level subject specific models (FSL's FEAT) were run by applying high-pass filtering (100 s, and subsequently the residuals and mean functional output were added together (FSL's res4d and mean_func). Following image preprocessing, lower-level subject specific models were run by regressing out average time courses over ventricles, white matter and subject-space wholebrain masks (using FSL's means). The residuals (res4d) and mean functional output (mean_func) from the FEAT output were subsequently combined to create a subject-specific preprocessed time series that was globally normalized and controlled for white matter and ventricle signals. This data was then used in a third series of lower-level subject-specific models that incorporated regressors of demeaned timeseries extracted from each seed region for each condition of interest. Thus, for each subject, we determined regions of coactivation to the specified seed within each contrast of interest, which was then combined at the group level with FLAME $1+2$ mixed effects modeling. Final results were 
determined through paired-sample t-tests for each contrast of interest $(F>N$ and $N>F$ ) using the TFCE method (FWE rate: $p<0.05,5,000$ permutations).

Additionally, whole brain exploratory analyses were carried out. To test whether controlling for age or sex would alter any of the reported results, both age and sex were regressed into neuroimaging task data and were not found to significantly relate to any reported regions.

\section{Questionnaires}

Questionnaires were incorporated into all functional analyses. First, questionnaire scores were used as regressors in higher level $\mathrm{ROI}$ functional analyses in the $\mathrm{F}>\mathrm{N}$ and $\mathrm{N}>\mathrm{F}$ contrasts (FWE-corrected, $\mathrm{p}<0.05)$. Additionally, regions displaying significant $\mathrm{FC}$ were masked using a $5 \mathrm{~mm}$ radius, and mean FC parameter estimates between regions were extracted for contrasts of interest and correlated with scores from each questionnaire. Finally, median splits of questionnaire scores were used for exploratory whole-brain group analyses, comparing Lower and Upper halves of each questionnaire for $\mathrm{F}>\mathrm{N}$ and $\mathrm{N}>\mathrm{F}$ contrasts. Areas of regional overlap between questionnaires following TFCE correction were masked using a $5 \mathrm{~mm}$ radius, and parameter estimated were extracted to calculate Cohen's d effect size between the Lower and Upper halves of significant results.

\section{Results}

\section{Behavioral Results}


Correlations of self-report questionnaires revealed that empathy was positively associated with worry $(r=0.30, p=0.04)$. Worry, rumination and anxiety were found to all be highly intercorrelated (worry and rumination: $r=0.64$, $p<0.001$; worry and anxiety: $r=0.60, p<0.001$; rumination and anxiety: $r=$ $0.70, p<0.001)$. Contrary to hypotheses, empathy was not directly associated with anxiety $(r=-0.04, p=0.77)$. However, empathy positively correlated with worry (PSWQ), while worry, rumination (RRS) and anxiety (STAI) were all highly intercorrelated (Table 3).

\section{Table 3}

Correlations between Behavioral Questionnaires.

\begin{tabular}{|c|c|c|c|c|}
\hline & TEQ & PSWQ & RRS total & STAI trait \\
\hline TEQ & 1.00 & & & \\
\hline PSWQ & $r={ }_{.04} .30, p=$ & 1.00 & & \\
\hline RRS total & $\begin{array}{c}r=.15, p= \\
.30\end{array}$ & $\mathrm{r}=.64, p<$ & 1.00 & \\
\hline STAI trait & $\begin{array}{c}r=-.04, p= \\
.77\end{array}$ & $r=.60, p<$ & $\begin{aligned} & r= .70, p< \\
& .001\end{aligned}$ & 1.00 \\
\hline
\end{tabular}

Note. Significant correlations are shown in bold.

$\mathrm{TEQ}=$ Toronto Empathy Questionnaire; PSWQ = Penn State Worry

Questionnaire; RRS = Ruminative Responses Scale; STAI = State Trait Anxiety Inventory

Given the lack of a direct effect between empathy anxiety, but a significant positive correlation between empathy and worry, an inconsistent mediational model was run to test for indirect effects. While classical mediation involves causal and directional relationships among variables, neither aspect is a necessary part of inconsistent mediation (i.e., suppression hypothesis). Instead, 
inconsistent mediation arises when the addition of a third variable actually increases the predictive validity between a predictor and outcome variable. Thus, significant indirect effects may exist even in the absence of a significant $c(X \rightarrow Y)$ pathway (MacKinnon et al., 2000; Zhao et al., 2010). Therefore, it was hypothesized that empathy and anxiety would be indirectly related through the process of worry.

Results from this analysis demonstrated a significant positive indirect effect between empathy and anxiety through worry, suggesting that higher empathy relates to higher worry, which in turn increases anxiety (TEQ: $p=.04$, PSWQ: $p<.001$, Bootstrapped 95\% Cl: [.055 - .597]). These effects were observed bidirectionally (STAI $\rightarrow$ PSWQ $\rightarrow$ TEQ), revealing an overall positive indirect relationship between empathy and anxiety, but only with the inclusion of worry. Furthermore, worry and rumination were included in a single model, which additionally revealed significant positive indirect effects between empathy and anxiety through both worry and rumination (TEQ: $p=.03$, PSWQ: $p=.01$, RRS: $p<.001$, Bootstrapped 95\% Cl: [.020 - .325]). This relationship was observed bidirectionally as well (STAI $\rightarrow$ RRS $\rightarrow$ PSWQ $\rightarrow$ TEQ), however, the positions of worry and rumination could not be interchanged as empathy only shared a direct relationship with worry (Figure 17). 

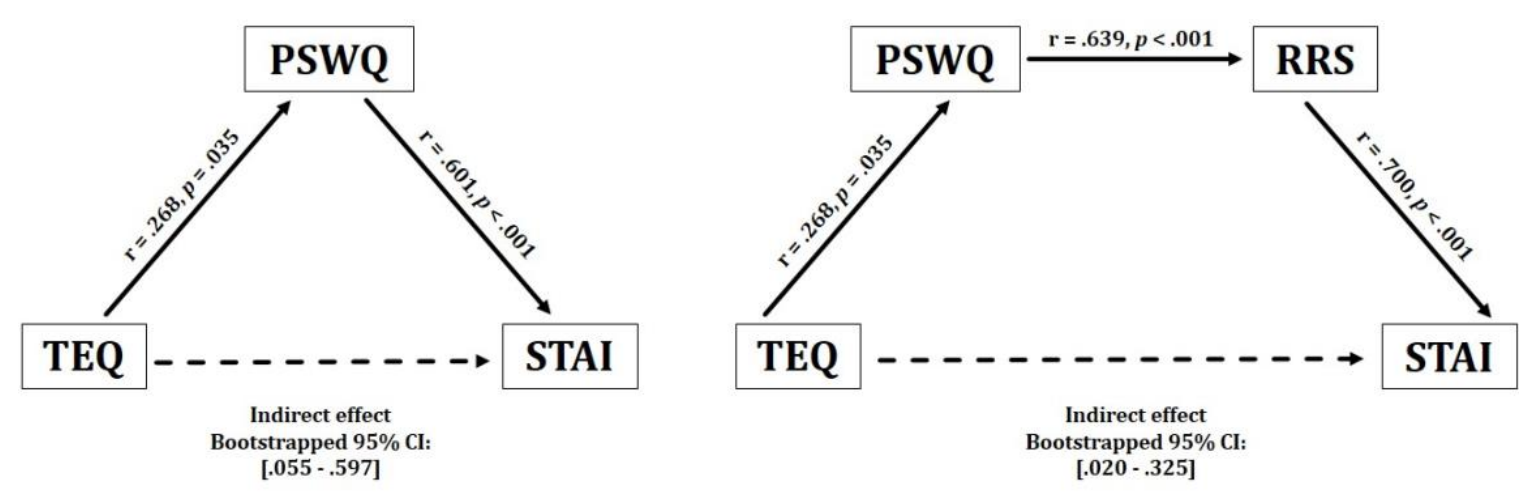

Figure 17: Behavioral results. Empathy demonstrated a significant positive indirect relationship with anxiety, through inconsistent mediation of worry. These effects were observed bidirectionally. Empathy also revealed a positive indirect relationship with anxiety through worry and rumination.

$T E Q=$ Toronto Empathy Questionnaire; $P S W Q=$ Penn State Worry

Questionnaire; RRS = Ruminative Responses Scale; STAI = State Trait Anxiety Inventory

\section{Neuroimaging Results}

Task- Related Region of Interest Analysis

Using an established socioemotional process network (TPJ, insula, mPFC, amygdala), we first evaluated regional activations when individuals were processing fearful and neutral human faces $(F>N$ and $N>F)$. Results revealed that greater left TPJ activation was related to $F>N$ faces, indicating enhanced face processing and supporting mentalizing. Greater bilateral anterior insula activation frequently associated with interoceptive awareness, was also related to $\mathrm{F}>\mathrm{N}$ faces. Finally, increased mPFC activation was related to $\mathrm{N}>\mathrm{F}$ faces, suggesting internal mentation, reflection or rumination (Figure 18; Table 4). No significant results were found related to amygdala. Upon further investigation, 
significant increased amygdala activations were observed in both main effects, in line with many studies that have shown amygdala activations for all face

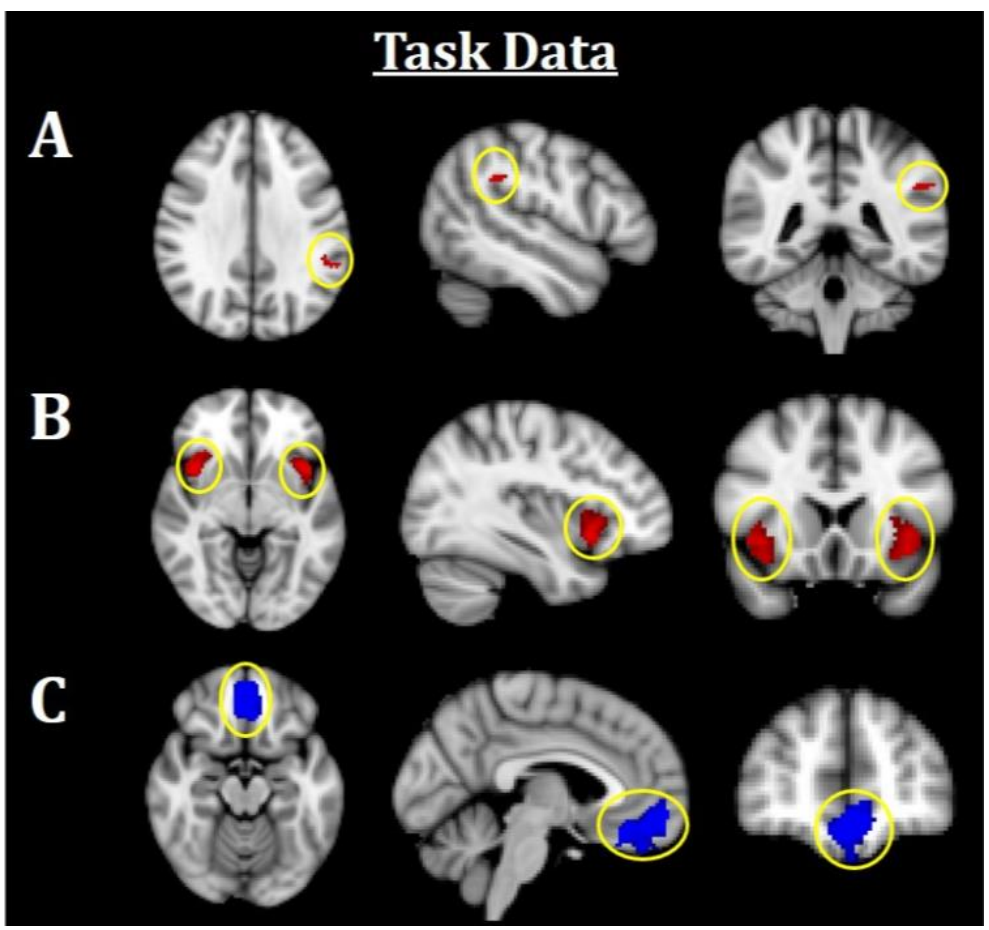
processing (Decety \& Sommerville, 2003; Todorov \& Engell, 2008), and thus differences did not emerge in either contrast. Nevertheless, these results support that integral components of a canonical socioemotional networks were activated during this Face Processing Task.

Figure 18: Neuroimaging Task Results. All results are FWE-corrected, $p<.05$. Red indicates Fearful $>$ Neutral $(F>N)$ faces and blue indicates Neutral $>$ Fearful $(\mathrm{N}>\mathrm{F})$ faces. A) Greater left TPJ activation was related to F>N. B) Greater bilateral insula activations related to $\mathrm{F}>\mathrm{N}$. C) Increased mPFC activation was related to $\mathrm{N}>\mathrm{F}$. TPJ = temporoparietal junction; $\mathrm{mPFC}=$ medial prefrontal cortex

Table 4: Neuroimaging Task Results, as shown in Figure 18.

\begin{tabular}{lccccc}
\hline Region & & $\begin{array}{c}\text { Peak t- } \\
\text { statistic }\end{array}$ & $\mathbf{x}$ & $\mathbf{y}$ & $\mathbf{z}$ \\
\hline Temporoparietal Junction (TPJ) & $\mathrm{L}$ & 3.98 & -54 & -44 & 32 \\
Insula & $\mathrm{R}$ & 4.20 & 40 & 12 & -6
\end{tabular}


Insula

Medial Prefrontal Cortex (mPFC)
L

$\mathrm{R} / \mathrm{L}$
3.99

4.42

$-38$

16

$-8$

Coordinates in MNI Space.

\section{Questionnaire Regressions}

Next, questionnaires measuring empathy, anxiety and worry were regressed into task data to investigate how these individual traits modulate processing within this socioemotional network. Higher empathy (TEQ) was found to be related to greater activation in the left TPJ for $F>N$ faces, suggesting that higher empathy is related to enhanced face processing and mentalizing, specifically for emotional faces. Regression of STAI revealed that higher trait anxiety was related to greater bilateral insula activations for $\mathrm{N}>\mathrm{F}$ faces.

Although on average, individuals showed increased insula activations for $\mathrm{F}>\mathrm{N}$ faces, these results demonstrate an interacting effect of anxiety, suggesting either that people higher in anxiety process the neutral faces as more negative, or exhibit prolonged interoceptive processing and emotional carry-over from the fearful faces. Similarly, higher worry (PSWQ) was found to be related to greater mPFC activation for $\mathrm{N}>\mathrm{F}$, indicating more internal mentation, and supporting the latter idea of continued processing of emotional stimuli into the neutral condition. Finally, regression with the RRS revealed that higher total rumination was associated with increased activations of bilateral TPJ, bilateral insula and mPFC for $\mathrm{N}>\mathrm{F}$ faces, an amalgamation of the three results from empathy, anxiety and worry (Figure 19; Table 5). 


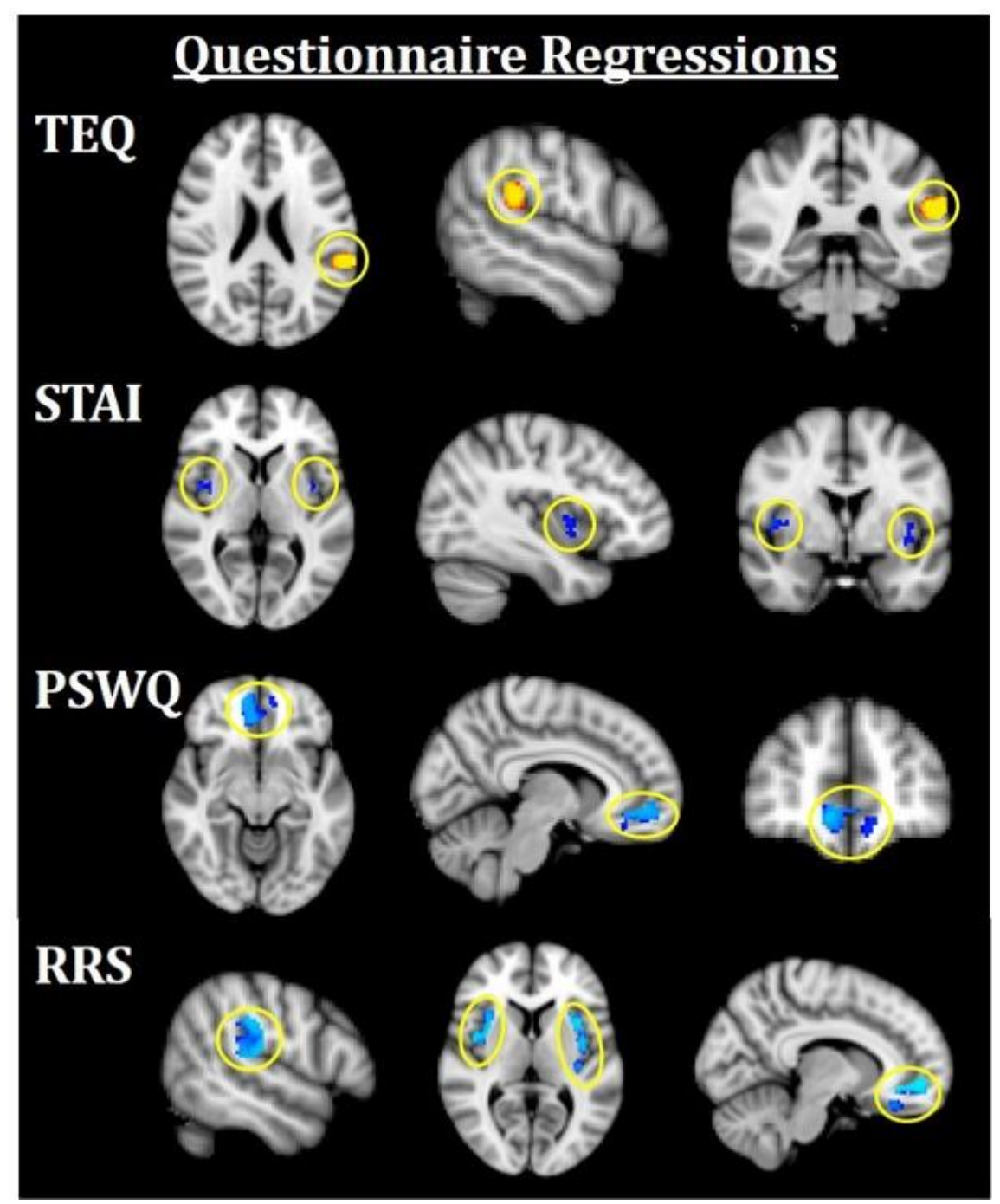

Figure 19: Neuroimaging results for questionnaire score regressions. All results are FWE-corrected, $p<.05$. Red/yellow indicates Fearful $>$ Neutral $(F>N)$ faces and blue indicates Neutral>Fearful $(\mathrm{N}>\mathrm{F})$ faces. TEQ: Higher empathy related to greater activation in the left TPJ (F>N). STAl: increased anxiety related to greater bilateral insula activations $(\mathrm{N}>\mathrm{F})$. PSWQ: More worry was related to greater activation in the MPFC (N>F). RRS total: Higher total rumination was related to grater activations in bilateral TPJ, bilateral insula, and $\mathrm{mPFC}(\mathrm{N}>\mathrm{F})$ $\mathrm{TEQ}=$ Toronto Empathy Questionnaire; PSWQ = Penn State Worry Questionnaire; STAI = State Trait Anxiety Inventory; RRS = Ruminative Responses Scale; TPJ = temporoparietal junction; $\mathrm{mPFC}=$ medial prefrontal cortex

Table 5: Neuroimaging results for questionnaire score regressions, as shown in Figure 19 


\begin{tabular}{|c|c|c|c|c|c|}
\hline Region & & $\begin{array}{l}\text { Peak t- } \\
\text { statistic }\end{array}$ & $\mathbf{x}$ & y & $\mathbf{z}$ \\
\hline \multicolumn{6}{|l|}{ TEQ } \\
\hline Temporoparietal Junction (TPJ) & L & 4.76 & -58 & -38 & 22 \\
\hline \multicolumn{6}{|l|}{ STAI } \\
\hline Insula & $\mathrm{R}$ & 3.34 & 46 & 0 & 2 \\
\hline Insula & L & 3.13 & -36 & 2 & -6 \\
\hline \multicolumn{6}{|l|}{$P S W Q$} \\
\hline Medial Prefrontal Cortex (mPFC) & $\mathrm{R} / \mathrm{L}$ & 3.57 & 10 & 50 & -8 \\
\hline \multicolumn{6}{|l|}{$R R S$} \\
\hline Temporoparietal Junction (TPJ) & $\mathrm{R}$ & 3.92 & 54 & -20 & 16 \\
\hline Temporoparietal Junction (TPJ) & L & 4.32 & -54 & -38 & 34 \\
\hline Insula & $\mathrm{R}$ & 4.04 & 32 & 16 & -2 \\
\hline Insula & L & 4.87 & -34 & 12 & 10 \\
\hline Medial Prefrontal Cortex (mPFC) & $R / L$ & 3.88 & 12 & 52 & -6 \\
\hline
\end{tabular}

Coordinates in MNI Space.

Because rumination revealed overlapping neural correlates with empathy, anxiety and worry, the behavioral data was revisited to assess rumination as a connecting point. Median splits of TEQ, STAI, and PSWQ were performed, and independent samples t-tests were calculated on degree of rumination between the Lower and Upper half groups of each questionnaire. Results showed that individuals in the Upper halves of empathy, anxiety and worry also had higher total rumination on average (TEQ: $t(47)=1.98, p=0.05$; STAI: $t(47)=7.59, p<$ 0.001; PSWQ: $t(47)=4.75, p<0.001)$. Rumination scores were then further 
divided into the three subscales-Reflection, Brooding and Depressive Rumination. Within the Reflection subscale, individuals in the Upper halves of empathy, anxiety and worry all had higher Reflective Rumination (TEQ: $t(47)=$ 2.00, $p=0.05$; STAI: $t(47)=2.54, p=0.0$ I PSWQ: $t(47)=2.56, p=0.01)$. However, for the Brooding (TEQ: $t(47)=1.18, p=0.25$; STAI: $t(47)=5.34, p<$ 0.001; PSWQ: $t(41)=4.93, p<0.001)$ and Depression subscales (TEQ: $t(47)=$ $1.73, p=0.09$; STAl: $t(47)=9.10, p<0.001$; PSWQ: $t(47)=4.75, p<0.001)$, only individuals in the Upper halves of anxiety and worry had significantly higher Brooding and Depressive Rumination (Figure 20).
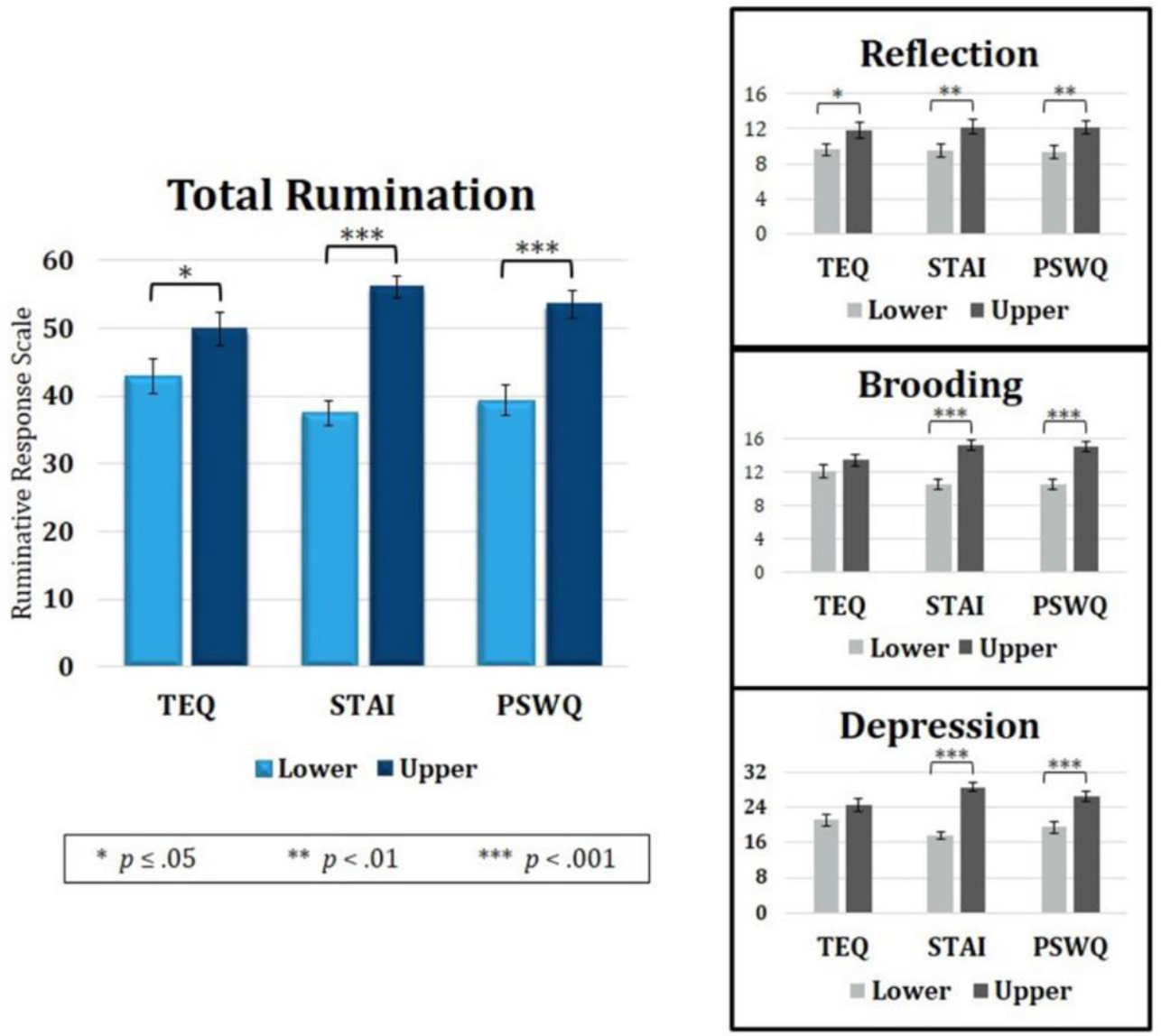
Figure 20: Median splits of TEQ, STAI and PSWQ showed that the Upper halves of all three questionnaires were related to higher total rumination (RRS total). Analysis of the three RRS subscales revealed that empathy, anxiety and worry were all related to higher Reflective Rumination. However, only the Upper halves of anxiety and worry were associated with higher Brooding and Depressive rumination. TEQ, Toronto empathy questionnaire; STAl, State-Trait Anxiety Inventory; PSWQ, Penn state worry questionnaire; RRS, Ruminative Responses Scale.

\section{Functional Connectivity}

Next, FC was performed between seeds and all regions comprising the socioemotional network. Significant increased FC was observed between the left amygdala and left insula (peak t-statistic $=4.72 ;-38,-6,-4$ ), as well as the left amygdala and left TPJ (peak t-statistic $=4.37 ;-52,-30,28$ ) for $\mathrm{F}>\mathrm{N}$ faces. This demonstrates that on average, individuals displayed increased communication between these regions when viewing emotional faces compared to neutral ones. However, when relating these findings to questionnaire scores, higher empathy, worry and total rumination were all related to increased connectivity between the left amygdala and left insula in the $\mathrm{N}>\mathrm{F}$ contrast (TEQ: $r=0.36, p=0.0 \mathrm{I}$;

PSWQ: $r=0.39, p=0.01$; RRS: $r=0.39, p=0.01$ ), suggesting not only enhanced communication between these regions in response to emotional faces, but sustained connectivity into the neutral condition for people higher on these traits. Similarly, increased total rumination was related to greater connectivity between the left amygdala and left TPJ in N > F (RRS: $r=0.24, p=0.04$; Figure 21). 


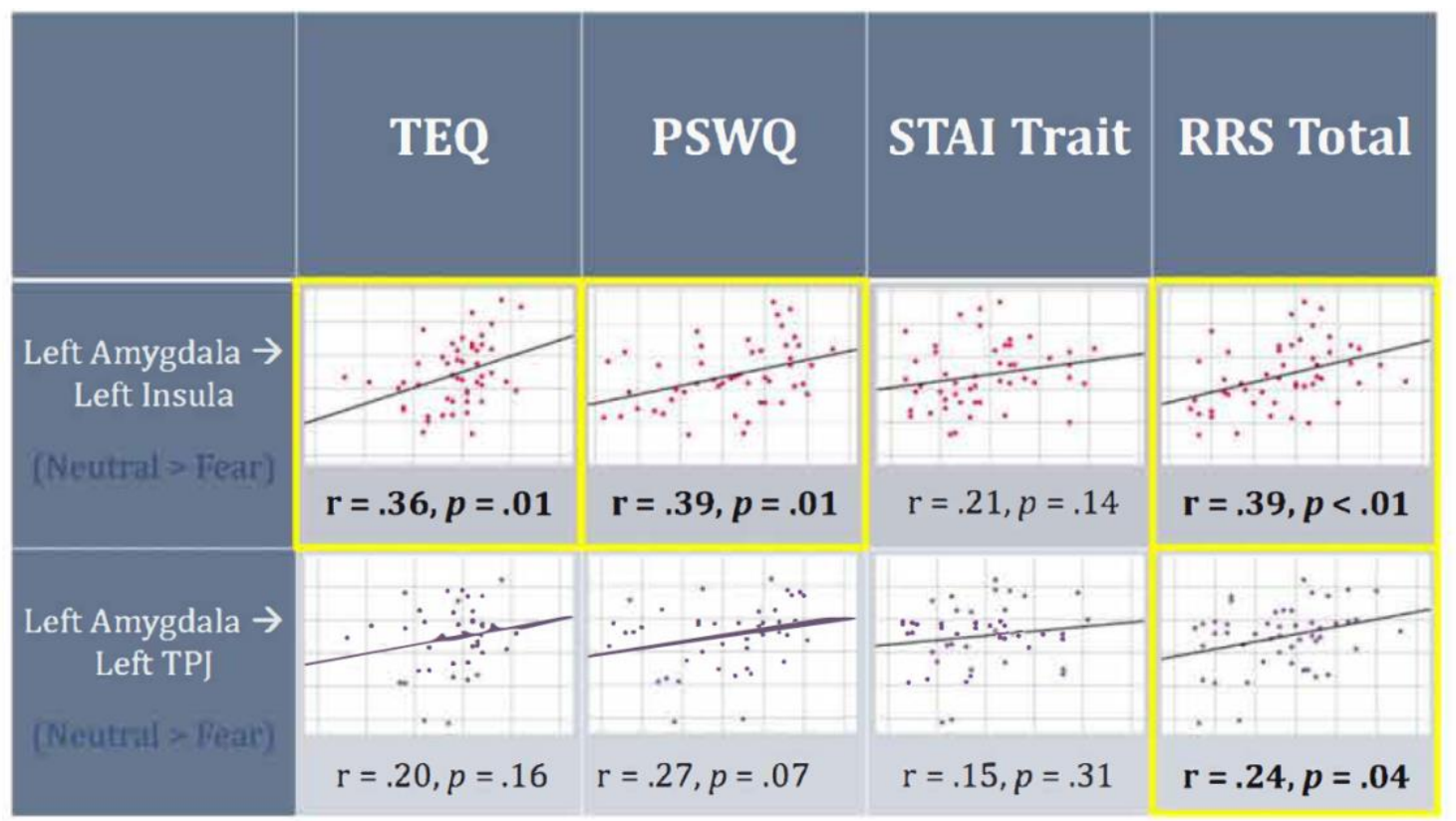

Figure 21: On average, individuals displayed increased functional connectivity (FC) between the left amygdala and left insula, as well as left amygdala and left TPJ for $\mathrm{F}>\mathrm{N}$ faces. Higher empathy, worry and rumination was found to be positively correlated with FC between the left amygdala and left insula for $\mathrm{N}>\mathrm{F}$ faces. Additionally, higher total rumination was related to increased connectivity between the left amygdala and left TPJ for N > F faces. TEQ, Toronto Empathy Questionnaire; STAl, State-Trait Anxiety Inventory; PSWQ, Penn State Worry Questionnaire; RRS, Ruminative Responses Scale; TPJ, temporoparietal junction.

\section{Whole-Brain Analysis}

Finally, we wanted to explore whether increased bottom-up processing or decreased top-down control seemed to be driving his putative emotional carryover from Fearful to Neutral trials. In other words, could we find either: (1) evidence for increased bottom-up processing in individuals high on these measures, suggesting greater sensitivity to social and emotional information; and/or (2) decreased top-down attentional control in individuals high on these 
measures, suggesting more difficulty in switching between emotional and nonemotional states? To test this, we performed an exploratory whole-brain analysis using the median split groups (with the addition of median split RRS total), comparing the Lower and Upper half groups of each questionnaire in the $\mathrm{F}>\mathrm{N}$ and $\mathrm{N}>\mathrm{F}$ contrasts. Results showed that in $\mathrm{F}>\mathrm{N}$, the Lower half groups were all associated with increased activations in frontoparietal attentional networks, with strong similarities and regional overlap between anxiety, worry and rumination [average Cohen's $d$ for Lower>Upper across questionnaires for left dorsolateral PFC $($ dIPFC $)=1.12$, and for left intraparietal sulcus $($ IPS $)=0.98]$. For empathy, one overlapping but non-significant cluster in the left dIPFC was observed (FWE-corrected, $p=0.10$ ). No significant results were found for $F>N$, Upper > Lower. Therefore, individuals on the Upper end of any or all of these measures appear to exhibit decreased top-down attentional control during the $\mathrm{F}>\mathrm{N}$ contrast, leading to both enhanced processing of emotional stimuli and prolonged processing into neutral situations (Figure 22). 


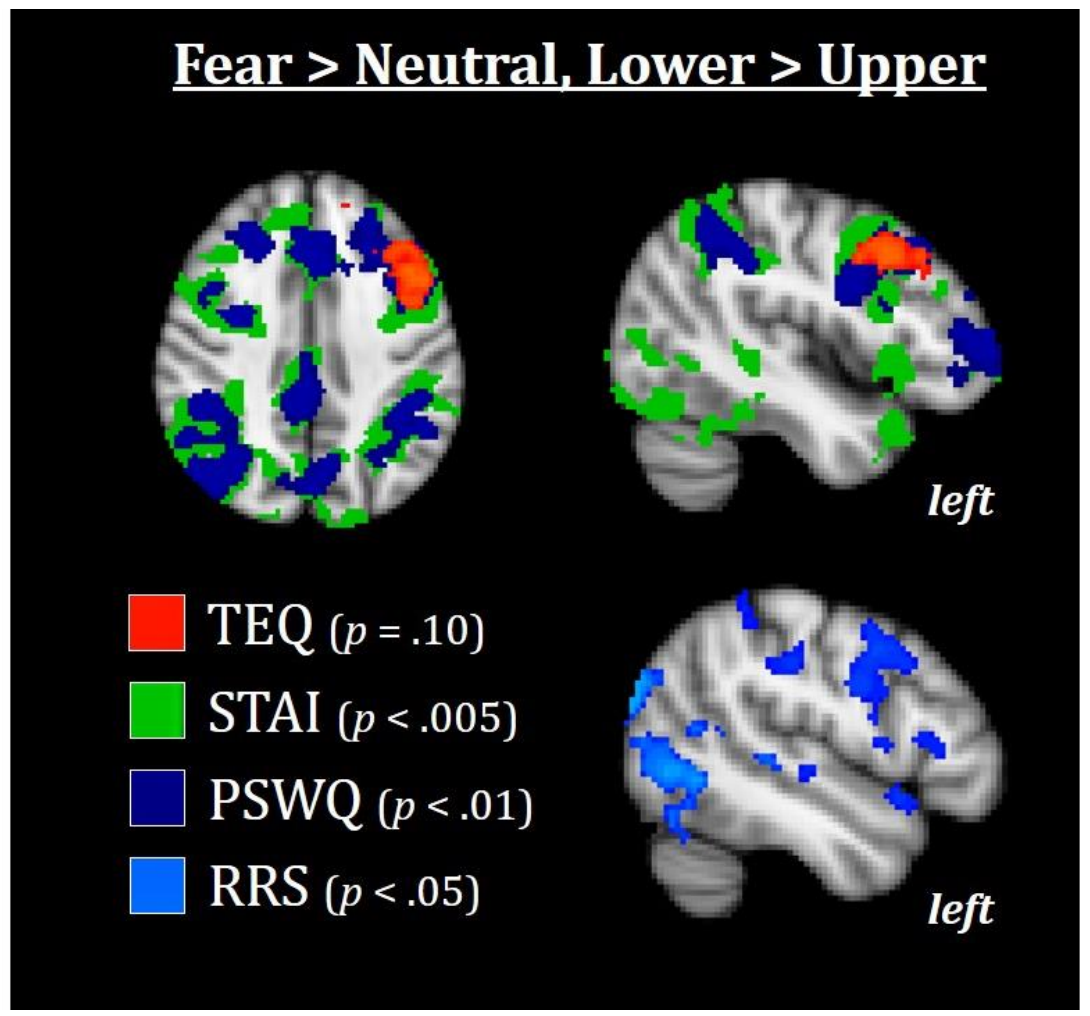

Figure 22: Median splits of TEQ, STAI, PSWQ, and RRS total revealed that the Lower halves of these traits were associated with enhanced activations in the frontoparietal attention network, with TEO exhibiting one overlapping but nonsignificant cluster in the left dIPFC. This increased top-down activation for individuals in the Lower halves of these traits may facilitate switching between emotional and non-emotional states. All results are FWE-corrected. TEQ, Toronto Empathy Questionnaire; STAl, State-Trait Anxiety Inventory; PSWQ, Penn State Worry Questionnaire; RRS, Ruminative Responses Scale; dIPFC, dorsolateral prefrontal cortex.

\section{Discussion}

The present study aimed to investigate the convergent neural correlates that may underlie a relationship between empathy and anxiety. We used an emotional Face Processing Task shown to reliably elicit activation from brain regions in a canonical socioemotional network, self-report questionnaires measuring empathy, anxiety and internally generated thought (i.e., worry and 
rumination), to examine how these traits were related to functional activation within, and communication between, regions comprising a socioemotional processing network. Our results show that empathy, anxiety and worry each engaged a different component of this socioemotional network, while rumination related to increased activations across all cortical regions in the network. Furthermore, higher empathy, worry and rumination were all associated with increased bottom-up connectivity, while anxiety was related to worry and rumination through decreased top-down attentional control. Taken together, our data demonstrate that in the context of socioemotional processing, empathy shares an indirect relationship with anxiety through the ruminative tendencies of worry.

Behaviorally, our results showed a positive correlation between empathy and worry, as measured by the TEQ and PSWQ, respectively. Empathy is closely tied to perspective-taking and mentalizing, which requires using one's own mental state to gain insight into another's thoughts or feelings. Comparatively, worry tends to be a future-oriented state that focuses on potential threats, but also represents an attempt at mental problem solving (Borkovec et al., 1983). Thus, this positive relationship between empathy and worry suggests that predispositions towards empathic arousal and perspective-taking may be natural to engage in problem-solving simulations on behalf of other individuals. Additionally, we observed a significant indirect relationship between empathy and anxiety through the process of worry, as well as through worry and rumination. 
While rumination shares many similarities to worry, it has been suggested that rumination is a process of "compulsively focusing attention on the symptoms of one's distress, and on its possible causes and consequences, as opposed to its solutions" (Nolen-Hoeksema, 1998). This idea both supports the observation that rumination is most closely related to anxiety and provides a scaffold for how empathy relates to worry, which in turn relates to rumination, and thus anxiety. Relationships to Functional Activation

Questionnaire regressions further revealed that each of these processesempathy, anxiety and worry-related to increased activations within different cortical regions comprising a socioemotional network. First, empathy was found to relate to increased activation in the left TPJ for $\mathrm{F}>\mathrm{N}$ faces. The TPJ is known to be a multimodal association area that integrates input from visual, auditory, somatosensory and limbic areas, and has reciprocal connection to the PFC, making it a central locus for processing multisensory information and cognitive aspects related to the self (Decety \& Lamm, 2006). In concert with the posterior STS, the TPJ is also thought to be specialized for processing faces and eye gaze (Blakemore, 2008), and thus aids in mentalizing (Bernhardt \& Singer, 2012). While the right TPJ is more commonly implicated in empathetic responses, studies have shown involvement of bilateral TPJ when participants read stories about character's mental states or false beliefs (Young et al., 2010), and specifically the left TPJ when participants imitated others (Decety \& Lamm, 2006). Taken together, this suggests that increased empathy may be related to 
enhanced face processing and mentalizing during the current study, particularly for emotional faces.

Regression of STAl trait revealed increased activations in bilateral anterior insula for Neutral trials, and similarly, higher worry was related to increased mPFC activation for Neutral trials, indicating that these traits are related to prolonged processing of emotional stimuli. Research suggests that the insula plays an important role in vicariously sharing emotions (Bernhardt \& Singer, 2012), as well as representing and integrating interoceptive and affective states (Craig, 2002, 2009). In fact, in one study looking at high and low degrees of alexithymia, the greater the individual's deficits in understanding their own emotions, the less insula activation they showed while empathizing with others in pain (Bird et al., 2010). Therefore, these increased activations in the anterior insula supports the notion that trait anxiety is related to increased emotional sensitivity and interoceptive awareness.

Furthermore, the mPFC has been linked to many aspects of social cognition, including monitoring one's own emotional state (Dvash \& ShamayTsoory, 2014), understanding emotional states of others (Amodio \& Frith, 2006), and internal mentation (Andrews-Hanna, 2011). Depressed participants show significantly greater activation in the mPFC than controls during experimentally induced rumination (Cooney et al., 2010) and individuals with generalized anxiety show sustained activation of the mPFC into resting epochs, which correlates with PSWQ scores (Paulesu et al., 2009). Together, this suggests that higher anxiety 
may promote continued interoceptive awareness into Neutral trials, while higher worry may play a role in cognitively processing those feelings (i.e., interoceptive processing and introspection). Finally, regression of RRS total showed that rumination was related to increased activations in bilateral TPJ, bilateral anterior insula, and mPFC for Neutral trials, suggesting that rumination is globally related to prolonged processing of emotional information, and moreover, alludes to relationships with empathy, anxiety and worry via overlapping neural correlates (summary in Figure 23).

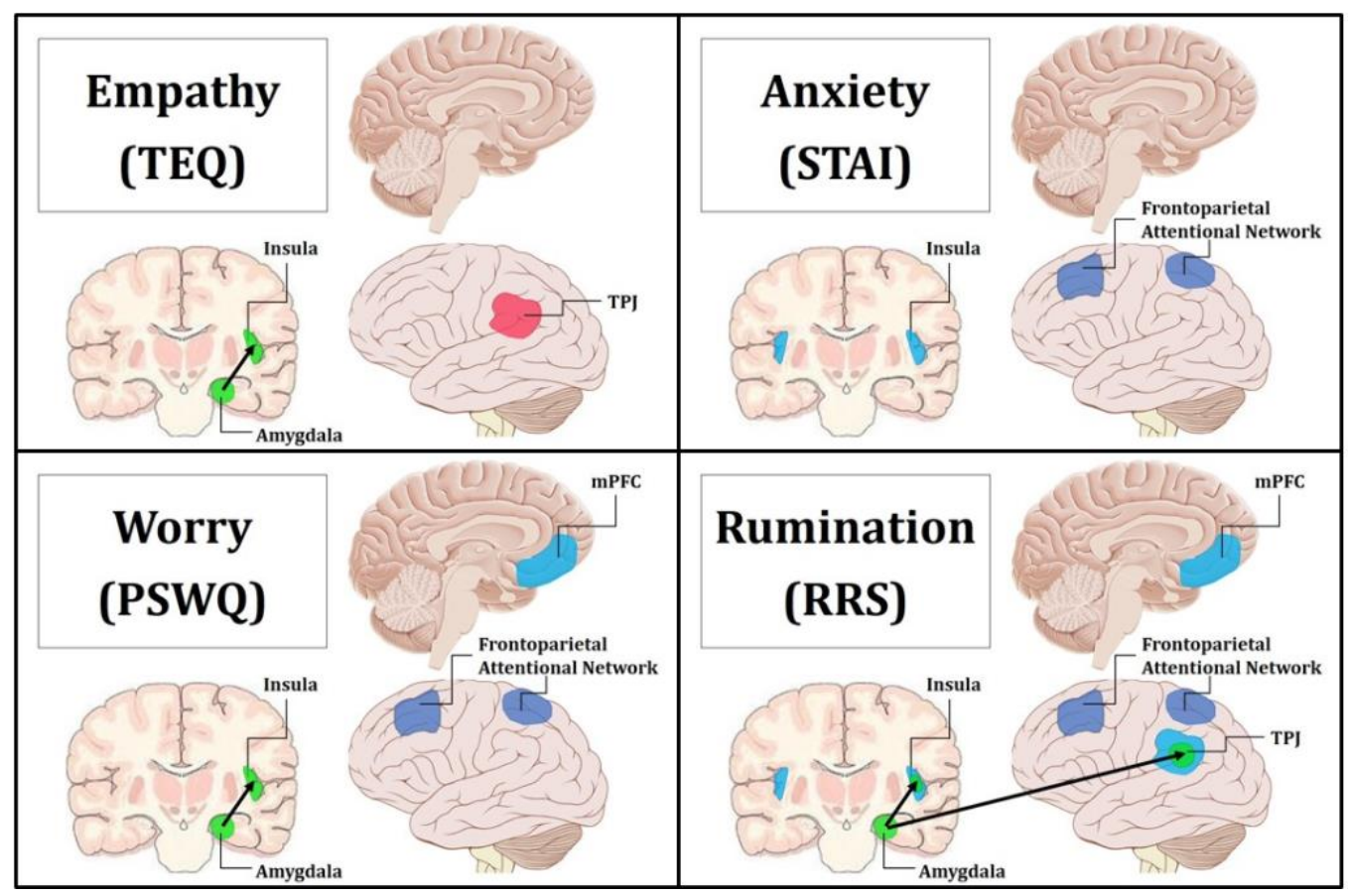

Figure 23: Summary figure of neuroimaging findings related to each trait. Behaviorally and neurally, empathy shared direct relationships with worry and rumination. Additionally, anxiety exhibited behavioral relationships and common neural correlates with worry and rumination. Empathy was found to only share a significant indirect behavioral relationship with anxiety through worry, and this is reflected in the convergent neural correlates of worry and rumination. Pink indicates increased regional activations for $F>N$. Blue indicates increased regional activations for $\mathrm{N}>\mathrm{F}$. Green with black arrows denotes increased FC for $\mathrm{N}>\mathrm{F}$. Purple represents decreased top-down attentional control for $\mathrm{F}>\mathrm{N}$ when 
comparing the Upper > Lower halves of questionnaires. Top Left: empathy was positively related to increased activation in the left TPJ for $\mathrm{F}>\mathrm{N}$, and increased FC between the left amygdala and insula for N > F. Top Right: anxiety was related to increased activations in bilateral insula for $N>F$, and decreased activations in the frontoparietal attentional network for Upper > Lower. Bottom Left: worry was related to increased activation in the mPFC for $N>F$, increased FC between the left amygdala and insula for $N>F$, and decreased activations in the frontoparietal attentional network for Upper > Lower. Bottom Right: rumination was related to increased activation in the MPFC, bilateral insula and bilateral TPJ for $\mathrm{N}>\mathrm{F}$, increased $\mathrm{FC}$ between the left amygdala and insula as well as the left amygdala and T PJ for $\mathrm{N}>\mathrm{F}$, and decreased activations in the frontoparietal attentional network for Upper > Lower.

\section{Empathy Relates to Reflection, Worry to Depressive Brooding}

Upon revisiting the behavioral data to further assess rumination as a connecting point between empathy and anxiety, median splits revealed that higher empathy, anxiety and worry were all related to higher total rumination. However, when assessing the rumination subscales, individuals in the Lower and Upper halves of empathy showed no differences on Brooding and Depression, suggesting that empathy is most closely tied to Reflective Rumination. In line with this finding, research indicates that self-reflection is positively correlated with perspective-taking and empathic concern (Joireman et al., 2002). On the other hand, the Lower and Upper halves of anxiety and worry showed significant differences in levels of Brooding and Depressive Rumination. Evidence suggests that the Brooding subscale is more pathological than Reflection, and Depressive Rumination is known to consist of items that overlap with measures of depression symptomatology (Treynor, 2003; Watkins \& Moulds, 2009). Furthermore, one study showed that rumination (measured separately from selfreflection) is in fact negatively related to perspective-taking, and is instead 
associated with personal distress (Joireman et al., 2002). Both worry and rumination have been described as unproductive and repetitive thought processes (Segerstrom et al., 2000) that exacerbate and prolong negative affect (McLaughlin \& Nolen-Hoeksema, 2011), and it is believed that this repetitive negative thinking is what increases vulnerability to multiple anxiety and depressive disorders (McEvoy et al., 2013). Therefore, while empathy shares a similar style of repetitive thinking and continued processing of emotional information through reflection, only worry and anxiety demonstrated a relationship with ruminative subscales associated with repetitive negative thinking typical of many clinical mood disorders. Because the relationship between empathy and anxiety was only found indirectly through worry, or worry and total rumination, this suggests that the tendency towards negative affect in worry and rumination may be a critical component necessary to link empathy to anxiety. Thus, empathy may be directly tied to worry through mentalizing and simulations of situational outcomes, but the indirect relationship to anxiety seems to rely on persistent negative affect induced by repetitive negative thinking found in the ruminative tendencies of worry.

\section{Neuroimaging Findings}

We next evaluated region-to-region $\mathrm{FC}$ within this canonical socioemotional network for the Face Processing Task and assessed the modulatory role these individual differences play. Interestingly, although no functional activation differences emerged in the amygdala, significant increased 
FC was observed between the left amygdala and left insula, as well as the left amygdala and left TPJ during Fear trials. When these findings were then related to our self-report measures, we found that higher empathy, worry and rumination were all related to increased connectivity between the amygdala and insula during Neutral trials, while rumination was additionally related to connectivity between the amygdala and TPJ for Neutral trials. Rumination of all types has been shown to relate to increased and sustained amygdala reactivity (Mandell et al., 2014), lasting throughout subsequent non-emotional trials (Siegle et al., 2002), and our results add that rumination may also be related to altered amygdala connectivity. Additionally, in support of our findings connecting empathy, worry and rumination through increased bottom-up connectivity, studies on autism spectrum disorders (ASDs), widely thought to be related to deficits in empathy, show decreased FC between the amygdala and insula (von dem Hagen et al., 2012), while resting state fMRI accounts report increased FC between these regions in relationship to state anxiety (Baur et al., 2013). Of interest, these FC results add to a growing list of curiously left-lateralized findings given the emotional nature of the task. However, worry, rumination and mentalizing are largely verbal or linguistic by nature (Fresco et al., 2002) and furthermore, participants were instructed to actively view and evaluate each face, hence promoting a more motivated "approach" response (Harmon-Jones et al., 2006), both of which are predominantly left-lateralized. Nevertheless, collectively with the results from regional functional activation, these findings reinforce the 
assertion of enhanced and prolonged emotional processing in association with these traits and outline a common neural mechanism linking empathy with worry/rumination (summary in Figure 23).

\section{Reduced Top-Down Attention Drives Prolonged Emotion Processing}

Finally, we pursued an exploratory whole-brain analysis to assess whether these neural commonalities were being driven by increased bottom-up processing, and/or decreased top-down control. Compared to the Upper halves, the Lower halves of anxiety, worry and rumination, but not empathy, were all associated with increased activations in the frontoparietal attentional network for fearful than neutral faces. This implies that in a naturalistic socioemotional setting, higher scores on these traits are all related to decreased top-down attentional control. Decreased activation in the dIPFC is thought to underlie disinhibition, allowing for sustained engagement of emotional-processing structures (Siegle et al., 2002). In support of this notion, higher brooding tendencies are associated with more errors when attempting to inhibit negative information (Vanderhasselt et al., 2011). Notably, these results demonstrate a common neural mechanism between anxiety, worry and rumination, but a dissociation from empathy, providing the indirect link between empathy and anxiety through worry that was previously observed behaviorally (summary in Figure 23).

Limitations and Future Directions 
It should be noted that all measures of empathy, anxiety, worry and rumination were collected through self-report questionnaires, which have their limitations. In light of this, future studies should continue to investigate the relationship between empathy and anxiety using performance measures, or other tasks using a broader range of stimulus types that cover more diverse emotional states. Future research should additionally extend these findings by untangling the relationship between anxiety, worry and rumination with cognitive and affective empathy, which display both behavioral and neurologically distinct mechanisms (Preckel et al., 2018). Furthermore, the present study was conducted as part of a much larger investigation on emotion regulation in young adults, and as such, only one questionnaire for each construct was collected. While a multi-method multi-trait approach would be ideal, we have attempted to demonstrate that the utilized questionnaires have strong internal reliability and discriminant validity in measuring their putative constructs. Additionally, all of our participants were considered psychologically healthy, and while the measured empathy/anxiety/worry/rumination scores covered a broad range it would be worthwhile to explore whether individuals with clinical disorders show corresponding results.

Previous literature has documented that many psychological disorders such as ASD (Baron-Cohen et al., 2005)and antisocial personality disorder (Blair, 2001) demonstrate decreased empathy and impaired social functioning, however other studies have also provided evidence that social information processing is 
often impaired in individuals with anxiety disorders (Luebbe et al., 2010). It is possible that by excluding the extreme ends of each spectrum, our observed linear relationships may not be capturing the whole picture. Finally, although our sample size was ample with nearly equal numbers of males and females, looking at gender differences was beyond the scope of this article. However, because there are known gender differences in empathy (Schulte-Rüther et al., 2008), emotional processing and prevalence of psychiatric disorders (Brody, 1997; McLean et al., 2011; McRae et al., 2008; Nolen-Hoeksema, 2001), future studies should explore how these neural mechanisms may vary by gender.

\section{Summary}

In conclusion, the present study sought to investigate neural commonalities that may support correspondence between trait empathy and anxiety in a healthy population. While a direct behavioral relationship was not observed between these traits, the results revealed indirect links between empathy and anxiety through the mediation of worry, and shared associations with higher self-reflection and ruminative thinking style. These findings, first seen behaviorally, were echoed through convergent neural correlates found in worry and rumination (Figure 23). First, empathy alone was related to worry and ruminative thinking through increased bottom-up communication of emotional processing regions (i.e., FC between the amygdala and insula). Further, results showed that worry and rumination shared commonalities with anxiety through decreased activations in the frontoparietal attentional network. Together this 
suggests that the enhanced and prolonged bottom-up processing of emotional information seen in empathy, in combination with the decreased top-down attentional control and repetitive negative thinking central to worry and rumination, may result in higher general anxiety in socioemotional encounters. 


\title{
CHAPTER V: A CLOSER LOOK AT EMOTION REGULATION
}

"He who does not control the signs of fear will experience fear in a greater degree, and he who remains passive when overwhelmed with grief loses his best chance of recovering elasticity of the mind"

\author{
Charles Darwin
}

\section{EXPERIMENT 4:}

Cortical Morphometry and Structural Connectivity Relate to Executive Function and Estradiol Level in Healthy Adolescents

Adolescence represents a dynamic developmental stage that corresponds with dramatic changes in brain architecture as it remodels itself to sustain the demands of a young adult physiology (Arain et al., 2013; Asato et al., 2010; Blakemore \& Choudhury, 2006; Paus, 2005). Previous studies consistently demonstrate a nonlinear change in cortical grey matter (GM) while in contrast, white matter (WM) exhibits a steady linear increase during adolescence (Arain et al., 2013; Giedd et al., 1999; Paus, 2005; Elizabeth R Sowell et al., 1999). This monumental neural reorganization driven by gonadal hormone exposure is thought to increase neural efficiency between the prefrontal cortex (PFC) and other posterior cortical structures. These structures subserve executive functions 
(EF), a set of processes necessary for seamless integration of top-down cortical control which forms the basis of goal-directed behavior (Blakemore \& Choudhury, 2006; Caballero et al., 2016; Giedd et al., 1999; Paus, 2005; Smolker et al., 2015; Elizabeth R Sowell et al., 1999).

Relationship between Executive Function and Cortical Morphometry

Despite EF's critical role in guiding future-oriented behavior, inconsistencies exist regarding the morphological features that support it during adolescence. While some studies demonstrate that increases in total cortical and PFC GM volume (GMV) relate to higher scores on working memory and response inhibition tests (Kharitonova et al., 2013; Mahone et al., 2009; Yurgelun-Todd, 2007); others show that GMV decreases in the PFC relate to increased ability to regulate emotion, better working memory capacity and higher scores on verbal memory tests (Caballero et al., 2016; Yurgelun-Todd, 2007). Similarly, higher IQ during adolescence is associated with cortical thinning of left superior orbitofrontal cortex and superior motor area, and higher bilateral hemispheric surface area (SA) (Schnack et al., 2014). Thus, bi-directional morphological results in relation to EF need not be interpreted as contradictory, instead they could possibly reflect the fact that GM maturation follows an inverted-U shape over development, peaking at different ages depending on the region (Ducharme et al., 2015; E R Sowell et al., 2001). Therefore, GM is considered closely related to maturation of a brain region (Crone, 2009; Giedd, 2004), suggesting that controlling for age is paramount when examining cortical GM in adolescent samples. 


\section{Relationship between Executive Function and Structural Connectivity}

In a more consistent pattern than GM maturation during adolescence, studies examining fractional anisotropy (FA; a WM integrity descriptor) during this period indicate relatively linear increases coinciding with improved EF performance. Specifically, increases in FA in the posterior corpus callosum during adolescence are associated with better working memory and IQ scores (Giedd, 2004; Giorgio et al., 2010; Nagy et al., 2004). Similarly, research indicates increased FA of fronto-temporal-subcortical WM tracts (inferior fronto-occipital longitudinal fasciculus (iFOF), superior longitudinal fasciculus (SLF), arcuate fasciculus and the cortico-spinal tract) support enhanced communication between disparate regions of the cortex and reflect increases in top-down cognitive control of behavior (Asato et al., 2010; Peper et al., 2015). While previous studies show specific changes in GM and FA and indicate relationships with some facets of $E F$, a comprehensive cortical morphometry and structural connectivity investigation concerning the full range of EF constructs is lacking.

\section{Measuring Executive Function in Children and Adolescents}

A tool often used to investigate multiple EF constructs and occasionally, their underlying neural substrates, is the Behavior Rating Inventory of Executive Function (BRIEF). This reliable and validated psychological battery is designed to measure EF behavior in children and adolescents (5-18 years) during everyday situations through behavioral observation (Clark et al., 2010). Initial factor analytic studies of the BRIEF support two robust indices: a Behavioral Regulation Index 
(BRI)—emphasizing inhibitory and emotional control (EC), and a Metacognition Index (MCl)—emphasizing working memory, planning, and strategic response preparation (Mahone et al., 2009). The sum of the two indices provides a Global Executive Composite (GEC), whereby elevated scores indicate more observed problems with EF behavior. Neurobiologically, research indicates that the BRIEF captures unique variance in predicting PFC development in children and adolescents (Mahone et al., 2009) and provides an economical port of entry to both behavioral regulation and cognitive issues that may in turn relate to cortical morphometry and structural connectivity measurements.

Relationship between Executive Function, Cortical Morphometry and Structural Connectivity

However, findings from the few studies that have investigated cortical morphometry in healthy, typically developing adolescents in relation to EF are inconsistent, likely due to the magnitude of change during adolescence. While some studies demonstrate that increased frontal GMV relates to decreased Working Memory and Emotional Control (Faridi et al., 2015; Mahone et al., 2009), others show the inverse pattern: decreased temporal lobe GMV relates to decreased Inhibition and Emotional Control (Faridi et al., 2015). Conversely, relationships between structural connectivity and the BRIEF during this variable period reflect an evident pattern: reductions in FA relate to decreased EF behavior. A variety of pediatric clinical populations exhibit reduced FA in temporal, frontal and corpus callosal regions in association with deficits on the 
GEC (Antshel et al., 2005; Herting et al., 2014; Wozniak et al., 2007). The sole study linking EF behavior with FA in a healthy adolescent sample investigated the frontal aslant tract (FAT), a newly discovered white matter tract which connects posterior inferior frontal gyrus (IFG) with the pre-supplementary and supplementary motor areas (pre-SMA and SMA), regions proposed to underlie inhibition. The study indicates the FAT develops in a protracted manner into late adolescence/early adulthood, and that right lateralization of this fiber pathway is significantly associated with decreased EF behavior as measured by the BRIEF (Garic et al., 2018). Taken together, scant evidence indicates that EF behavior is associated with both GM and FA changes during childhood and adolescence, yet the results are conflicting. Therefore, a comprehensive cortical morphometry and structural connectivity study using the BRIEF to assess EF behavior within a healthy sample of adolescents can help clarify previous findings.

Relationship between Executive Function, Cortical Morphometry, Structural Connectivity and Estradiol

During this developmental period of high flux, the hormone estradiol, the predominant estrogen, has been shown to have a significant impact on the structural reorganization of the prefrontal cortex (McCarthy, 2008; Nguyen et al., 2013), a crucial region underlying EF (Yuan \& Raz, 2014). The hormone has complex effects in the two genders however, because estrogen receptor distribution in the prefrontal cortex varies (Cooke et al., 2017; Gillies \& McArthur, 2010). Therefore, estradiol may have both similar and different (sometimes 
opposite effects) due to underlying brain dimorphisms. Nonetheless, this hormone influences cognitive function through complex interactions with dopaminergic and oxytocinergic systems that govern EF (Kuhn et al., 2010; Steinberg, 2005), the description of which is not within the scope of this paper. The complex, menstrualphase dependent evidence from studies in adult women points to estradiol level playing both a facilitative and/or hindering role in cognitive function. Some studies report higher levels of circulating estradiol being associated with improved working memory performance (Hampson \& Morley, 2013), while others show increased estradiol had a negative impact on general processing speed, working memory performance (Sommer et al., 2018), and slower response times and decreased accuracy on EF tasks that were instead related to progesterone level during the luteal phase (Hidalgo-Lopez \& Pletzer, 2017). Alongside the adult literature, morphometric studies in young adults demonstrate increased circulating levels of estradiol are associated with cortical thinning of the IFG (Witte et al., 2010), a region linked to self-regulation (Smolker et al., 2015; Witte et al., 2010). Structural connectivity evidence points to elevated estradiol level influencing decreases in FA, which is associated with reduced behavioral control during early pubertal development (Peper et al., 2015). Elevated estradiol level in adolescent girls shows a negative relationship with right angular gyrus (AG) and the superior longitudinal fasciculus (SLF) FA, a brain region and a WM tract involved in attention, spatial and social cognition (Herting et al., 2012). However, scant 
evidence between the relationship between EF behavior, estradiol and specific brain changes exists.

Aims

Therefore, the present study aimed to comprehensively investigate the relationship between EF behavior (as measured by the BRIEF questionnaire) and estradiol level, individually and interactively on cortical morphometry and FA in a healthy adolescent sample. Specifically, the aims were to examine the relation between: 1) the BRIEF and estradiol level, 2) the BRIEF, cortical morphometry and FA, 3) estradiol level, cortical morphometry and FA, and 4) any interaction between the BRIEF and estradiol level with cortical morphometry and FA.

\section{Hypotheses}

We hypothesized based on the limited literature findings that: 1) EF behavior and estradiol level will be inversely related, 2) Decreased EF behavior will relate to decreased GMV of the LPFC and decreased FA of WM tracts subserving EF (iFOF/SLF) 3) Increased estradiol level will relate to decreased FA and cortical morphometry of the LPFC and, 4) increased estradiol level combined with decreased EF behavior would subsequently exacerbate these previous findings. This comprehensive study, therefore, investigated how individual differences in EF behavior and estradiol level relate to variation in aspects of cortical morphometry and FA in a healthy, adolescent sample. 


\section{Methods}

\section{Participants}

Cross-sectional data were obtained from the Pediatric MRI Data Repository (Release 4.0) of the NIH MRI Study of Normal Brain Development, a project developed to characterize healthy brain maturation in relation to behavior in a large, multisite study (Evans, 2006). This multi-center project conducted epidemiologically based recruitment of a large, demographically balanced sample across a wide age range, using strict exclusion factors and comprehensive clinical/behavioral measures. A mixed cross-sectional and longitudinal design was used to create an MRI/clinical/behavioral database from approximately 500 children, aged 7 days to 18 years, to be shared with researchers and the clinical medicine community. Using a uniform acquisition protocol, data were collected at six Pediatric Study Centers and consolidated at a Data Coordinating Center. Enrolled subjects underwent a standardized protocol to characterize neurobehavioral and pubertal status. The data was demographically representative of the U.S. population in terms of variables including gender, race, and socioeconomic status (Waber et al., 2012). Exclusion criteria included but were not limited to $I Q<70$, history of medical illness with CNS implications, and any Axis I psychiatric disorder (other than simple or social phobia, adjustment disorder, oppositional defiant disorder, enuresis, encopresis, or nicotine dependency; see (Waber et al., 2007) for a complete list of inclusion and exclusion criteria). Participants underwent brain MRIs and extensive neuropsychological 
testing on up to three occasions at two-year intervals. For the purposes of this report, a sample of 55 participants (age range 7-18) with cross sectional data (1 timepoint) was selected with structural imaging data ( $\mathrm{T} 1)$, diffusion tensor imaging data (DTI), behavioral (BRIEF) and hormonal data (estradiol). 7 participants were missing estradiol data, therefore they were not included in subsequent analyses involving estradiol. Collection site was treated as a nuisance factor in all subsequent analyses.

Behavior Rating Inventory of Executive Function (BRIEF)

The BRIEF was completed on the same day as the scan by a parent or guardian that had contact with the child within the prior 6 months. The 86 -item questionnaire takes approximately 10 minutes to administer and can be administered and scored by a research assistant. The test was divided into the Behavioral Regulation Index (BRI) which comprised: Inhibit, Shift, Emotional Control subscales, and the Metacognition Index (MCl) which comprised: Initiate, Working Memory, Plan/Organize, Organization of Materials, and Monitor subscales. A higher score on each of the subscales signified decreased EF behavior. Subscales (Table 6) were used for further correlation and regression analyses with cortical, FA and hormonal measurements, controlling for age and gender. Multiple comparison correction was carried out using the Benjamini \& Hochberg, 1995 procedure (Benjamini \& Hochberg, 1995), controlling the false discovery rate (FDR) at $p<.05$.

\begin{tabular}{|l|l|}
\hline Inhibit & Ability to control impulses (inhibitory control) and to stop
\end{tabular}
engaging in a behavior 


\begin{tabular}{|l|l|}
\hline Shift & $\begin{array}{l}\text { Ability to move freely from one activity or situation to another; } \\
\text { to tolerate change; to switch or alternate attention }\end{array}$ \\
\hline $\begin{array}{l}\text { Emotional } \\
\text { Control }\end{array}$ & Ability to regulate emotional responses appropriately \\
\hline Initiate & $\begin{array}{l}\text { Ability to begin an activity and to independently generate } \\
\text { ideas or problem-solving strategies. }\end{array}$ \\
\hline $\begin{array}{l}\text { Working } \\
\text { Memory }\end{array}$ & $\begin{array}{l}\text { Ability to hold information when completing a task, when } \\
\text { encoding information, or when generating goals/plans in a } \\
\text { sequential manner. }\end{array}$ \\
\hline Plan/Organize & $\begin{array}{l}\text { Ability to anticipate future events; to set goals; to develop } \\
\text { steps; to grasp main ideas; to organize and understand the } \\
\text { main points in written or verbal presentations. }\end{array}$ \\
\hline $\begin{array}{l}\text { Organization } \\
\text { of Materials }\end{array}$ & $\begin{array}{l}\text { Ability to put order in work, play and storage spaces (e.g., } \\
\text { desks, lockers, backpacks, and bedrooms). }\end{array}$ \\
\hline Monitor & $\begin{array}{l}\text { Ability to check work and to assess one's own performance; } \\
\text { ability to keep track of the effect of one's own behavior on } \\
\text { other people. }\end{array}$ \\
\hline
\end{tabular}

Table 6: BRIEF subscale descriptions

Physiological Measures

\section{Estradiol}

At each visit during the assessment day, all subjects provided two separate 1-3cc samples of saliva at two time points between 12 and $6 \mathrm{pm}$. The maximum range for the collection of the two hormonal time points was 7 hours and $40 \mathrm{~min}$. Saliva was collected while the subject was relaxed and not after potentially stressful procedures (e.g. MRI). Samples were collected, stored at -20 to $-80^{\circ} \mathrm{C}$, and shipped in batches from each site to UCLA. Samples were assayed by published RIA methods for estradiol in Dr. McCracken's laboratory at UCLA. Estradiol level was moderately skewed $0.89(\mathrm{SE}=0.34)$ and kurtosis $0.10(\mathrm{SE}=$ 0.67), so appropriate log10 transformation was performed. Log10-transformed estradiol levels (skewness $-0.05(\mathrm{SE}=0.34)$ and kurtosis $-0.985(\mathrm{SE}=0.67))$ were 
included as regressors in all subsequent multiple regression analyses. An average of the two-time points was used for further correlation and regression analyses, controlling for time of collection. Due to the strong hormonal impact on pubertal status, the sample $(\mathrm{N}=55)$ was divided into gender-specific pre (14 F, $9 \mathrm{M})$ and

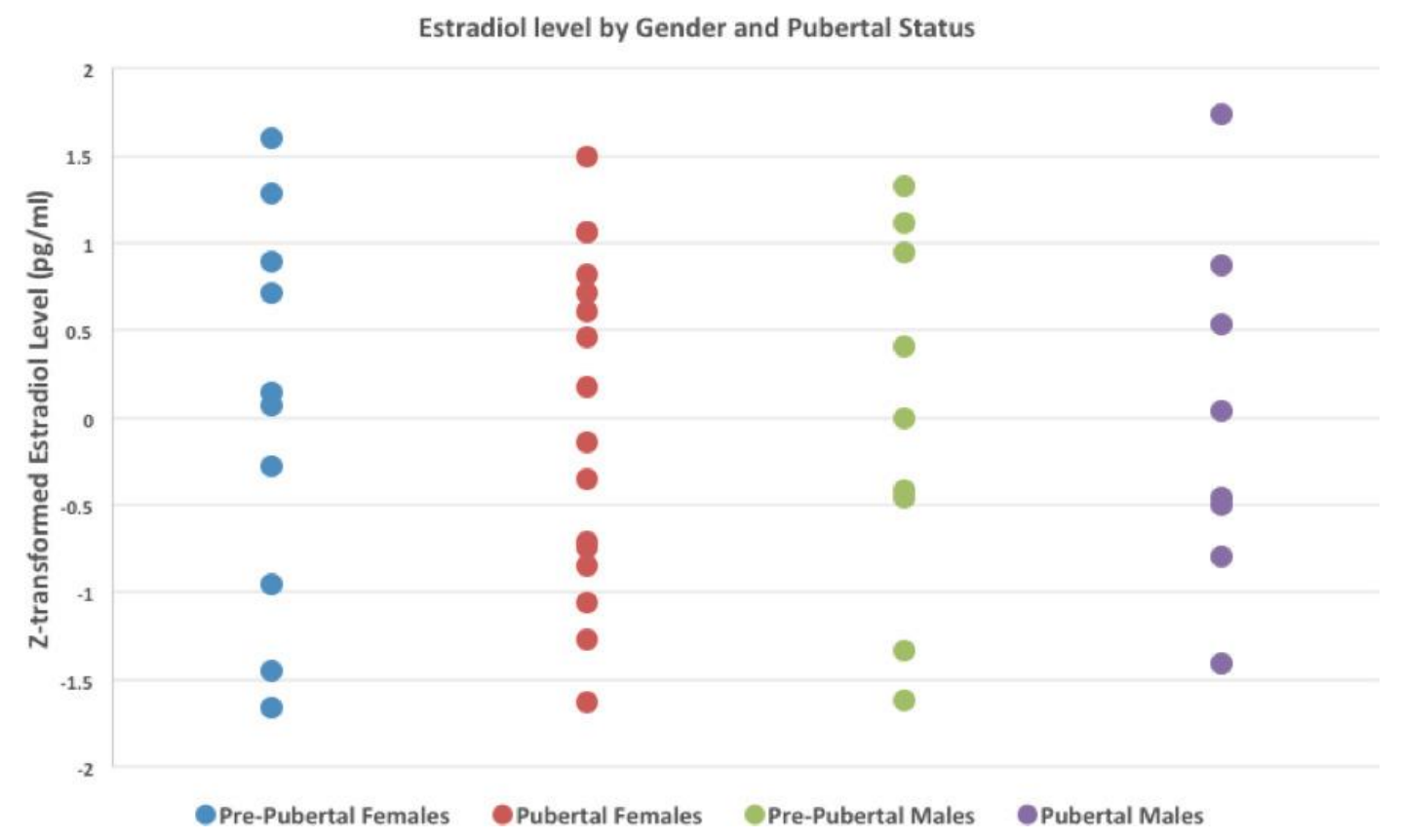

post (16 F, $8 \mathrm{M}$ ) pubertal groups (as indicated by the Tanner Stage) and use as a categorical variable to measure the impact of puberty on EF (as indicated by the BRIEF subscales), cortical morphometry and structural connectivity. We tested the multiple regression slopes ( $\beta$-weights) of the pre and post pubertal groups (Figure 24) in the cortical morphometry analyses (including BRIEF subscale-by-estradiol interactions). We additionally performed independent samples T-tests comparing pre and post-pubertal groups by BRIEF subscales, estradiol level and structural connectivity.

Figure 24: Normalized estradiol values divided by gender and pre- and postpuberty groups 


\section{Neuroimaging Methods}

Imaging Data Acquisition

\section{Cortical Morphometry}

High-resolution, T1-weighted images were acquired using a 1.5-Tesla RI scanner from General Electric (GE) or Siemens Medical Systems (Siemens) (Evans, 2006). Imaging data were obtained for each participant on the day of or within a maximum of 28 days of psychometric testing at each visit. GE: SPGR, $T R=22 \mathrm{~ms}, T E=10-11 \mathrm{~ms}$, flip angle $=30 \mathrm{deg}$, sagittal orientation, $F o V=250 \times 250$, Matrix $=256 \times 256 \times 124-180$ slices, 1-1.5 variable $\mathrm{mm}$ slice thickness. Siemens: SPGR, TR=25ms, TE=11 ms, flip angle=30deg, sagittal orientation, $F o V=256 X 256$, Matrix=256X256X160-180, 1mm slice thickness.

\section{Diffusion Tensor Imaging (DTI)}

Data were acquired at a subset of sites (Boston, Cincinnati, Philadelphia, St. Louis) with a diffusion-encoded multislice spin echo EPI sequence. To avoid orientation bias, data were acquired on a $3 \times 3 \times 3 \mathrm{~mm}$ matrix covering the entire brain with straight axial slices. GE: diffusion encoded spin echo EPI, TR=3s, $\mathrm{TE}=$ minimum full, flip angle=90deg, axial orientation, $\mathrm{FoV}=192$ if brain $19 \mathrm{~cm}$, Matrix=64X64X48; if brain larger than $19 \mathrm{~cm} \mathrm{FoV=384} \mathrm{with} \mathrm{Matrix=128X128X60,}$ 4 series of 6 diffusion directions $b=1000$. Siemens: diffusion encoded spin echo $E P I, T R=3 s, T E=m i n i m u m$ full, flip angle $=90$ deg, axial orientation, $F o V=192$ if 
brain less than $19 \mathrm{~cm}$ with Matrix $=64 X 64 X 48$; if brain larger than $19 \mathrm{~cm}$ FoV $=384$ with Matrix $=128 \times 128 \times 60,4$ series of 6 diffusion directions $b=1000$.

Image Processing

\section{Surface Based Morphometry (SBM)}

Cortical reconstruction and volumetric segmentation was performed with the Freesurfer image analysis suite (v5.6.0), which is documented and freely available for download online (http://surfer.nmr.mgh.harvard.edu/). The technical details of these procedures are described in prior publications (Bruce Fischl et al., 1999). Briefly, this processing includes motion correction and averaging (Reuter et al., 2010) of volumetric T1-weighted images, removal of non-brain tissue using a hybrid watershed/surface deformation procedure (Ségonne et al., 2004), automated Talairach transformation, intensity normalization (Sled et al., 1998), tessellation of the grey matter white matter boundary, automated topology correction (B. Fischl et al., 2001; Segonne et al., 2007), and surface deformation following intensity gradients to optimally place the grey/white and grey/cerebrospinal fluid borders at the location where the greatest shift in intensity defines the transition to the other tissue class (Dale \& Sereno, 1993; B Fischl \& Dale, 2000; Bruce Fischl et al., 1999). Once the cortical models are complete, a number of deformable procedures were carried out for further data processing and analysis including surface inflation, registration to a spherical atlas which utilized individual cortical folding patterns to match cortical geometry across subjects, parcellation of the cerebral cortex into units based on gyral and sulcal structure 
and creation of a variety of surface-based data including maps of cortical volume, surface area (SA), thickness, curvature, sulcal depth, and local gyrification index (Desikan et al., 2006; Bruce Fischl et al., 1999, 2004). The resulting probability maps were input into a general linear model (GLM) evaluating regressions between all vertices and BRIEF subscales, estradiol level, as well as the BRIEF subscale interaction with estradiol level (calculated by multiplying the raw BRIEF score with the estradiol level) controlling for age, gender, time of estradiol collection (when estradiol was present in the analysis), intracranial volume (ICV) and collection site. Vertex-wise threshold was set at $p<0.001$ level. Cluster-wise threshold was corrected for at $p<0.05$ level using non-parametric permutation testing with Monte Carlo simulation.

\section{DTI Data}

DTI images were acquired from the NIHPD database already brain extracted, corrected for eddy-current and EPI distortion. DTI images were then further processed using FSL's (v5.0.8, https://fsl.fmrib.ox.ac.uk/fsl/fslwiki/FSL) FDT toolbox (http://fsl.fmrib.ox.ac.uk/fsl/fslwiki/ FDT) (Behrens et al., 2003). A diffusion tensor model was fit at each voxel, resulting in FA images. FA images from all subjects were registered to an MNI $1 \mathrm{~mm}$ skeletonized DTI template using FNIRT, a non-linear registration tool in FSL. FA values for each subject were then extracted from masks of WM tracts created by the John Hopkins University (JHU) WM atlas: forceps major and minor and bilateral: anterior thalamic radiation, corticospinal tract, cingulum bundle cingulate region $(\mathrm{CBC})$, cingulum bundle 
hippocampal region ( $\mathrm{CBh}$ ), inferior fronto-occipital fasciculus (iFOF), inferior longitudinal fasciculus (ILF), superior longitudinal fasciculus (SLF) and uncinate fasciculus. FA values were used in further regression analyses using Pearson correlation coefficient, in SPSS (IBM SPSS Statistics for Macintosh, Version 22.0) using bootstrapping and permutation testing (3,000 simulations) to adjust for small sample size. All analyses were controlled for age, gender and collection time when estradiol was a included as a variable. Multiple comparison correction was carried out using the Benjamini \& Hochberg, 1995 procedure, controlling the false discovery rate $(F D R)$ at $p<.05$. Significant tracts were isolated using the tract visualization program TrackVis (Ruopeng Wang and Van J. Wedeen at Martinos Center for Biomedical Imaging, Massachusetts General Hospital, Charlestown, Mass., USA; trackvis.org, Version 0.6.1). A 3 mm-diameter disk-shaped ROI was placed in the tract of interest, which allowed for full capture of fibers of interest.

\section{Results}

\section{Behavioral/Hormonal Results}

No significant results were found when investigating the relationship between EF behavior and estradiol level (Table 7A). Given the notable effects of age/gender in the sample, a separate analysis investigating the unique role of age/gender on the relationship between BRIEF subscales and estradiol level was explored (i.e. not controlling for age and gender, Table 7B). This resulted in no 
significant findings, suggesting age and gender do not have an impact on executive function (EF) behavior and estradiol level in this sample.

\begin{tabular}{|c|c|c|c|c|c|c|c|c|}
\hline \multirow[b]{3}{*}{$\begin{array}{l}\text { Positive Pubertal } \\
\text { Status }\end{array}$} & \multicolumn{2}{|c|}{$\begin{array}{l}\text { Male } \\
(\mathrm{N}=18)\end{array}$} & \multicolumn{2}{|c|}{$\begin{array}{l}\text { Female } \\
(\mathrm{N}=30)\end{array}$} & \multicolumn{4}{|c|}{$\begin{array}{c}\text { Correlation of estradiol with EF } \\
\text { Behavior }\end{array}$} \\
\hline & \multirow{2}{*}{\begin{tabular}{|l} 
\\
\end{tabular}} & \multirow{2}{*}{\begin{tabular}{|l|} 
S \\
D \\
\end{tabular}} & \multirow{2}{*}{$\begin{array}{l}\mathrm{M} \\
17\end{array}$} & \multirow[t]{2}{*}{ SD } & \multicolumn{2}{|c|}{$\begin{array}{c}\text { A. Not } \\
\text { Corrected for } \\
\text { Age/Gender }\end{array}$} & \multicolumn{2}{|c|}{$\begin{array}{l}\text { B. Corrected } \\
\text { for Age/Gender }\end{array}$} \\
\hline & & & & & & & & \\
\hline Age (years) & $\begin{array}{r}13 \\
.0 \\
2\end{array}$ & $\begin{array}{r}3 . \\
02\end{array}$ & $\begin{array}{r}13 . \\
75\end{array}$ & $\begin{array}{r}3.5 \\
6\end{array}$ & $r$ & $p$-value & $r$ & $p$-value \\
\hline $\begin{array}{l}\text { log-estradiol } \\
\text { level (pg/ml) }\end{array}$ & $\begin{array}{r}0 . \\
79 \\
\end{array}$ & $\begin{array}{r}0 . \\
24\end{array}$ & $\begin{array}{r}0.8 \\
6\end{array}$ & $\begin{array}{r}0.2 \\
9 \\
\end{array}$ & 1 & {$[-]$} & 1 & {$[-]$} \\
\hline $\begin{array}{l}\text { BRIEF Behavior } \\
\text { Regulation }\end{array}$ & $\begin{array}{r}34 \\
.2 \\
9\end{array}$ & $\begin{array}{r}6 . \\
07\end{array}$ & $\begin{array}{r}36 . \\
07\end{array}$ & $\begin{array}{r}6.7 \\
9\end{array}$ & 0.03 & 0.86 & 0.03 & 0.96 \\
\hline $\begin{array}{l}\text { BRIEF Emotional } \\
\text { Control }\end{array}$ & $\begin{array}{r}12 \\
.1 \\
8\end{array}$ & $\begin{array}{r}2 . \\
19\end{array}$ & $\begin{array}{r}13 . \\
4\end{array}$ & $\begin{array}{r}3.1 \\
8\end{array}$ & -0.05 & 0.74 & -0.05 & 0.52 \\
\hline $\begin{array}{l}\text { BRIEF Global } \\
\text { Executive } \\
\text { Composite }\end{array}$ & $\begin{array}{r}98 \\
.8 \\
8\end{array}$ & $\begin{array}{r}17 \\
.2 \\
3\end{array}$ & $\begin{array}{r}98 . \\
87\end{array}$ & $\begin{array}{r}17 . \\
57\end{array}$ & 0.12 & 0.45 & 0.12 & 0.43 \\
\hline BRIEF Initiate & $\begin{array}{r}11 \\
.5 \\
\end{array}$ & $\begin{array}{r}2 . \\
5\end{array}$ & $\begin{array}{r}11 . \\
62 \\
\end{array}$ & $\begin{array}{r}2.0 \\
3 \\
\end{array}$ & 0.28 & 0.07 & 0.28 & 0.11 \\
\hline BRIEF Inhibition & $\begin{array}{r}12 \\
.2 \\
2 \\
\end{array}$ & $\begin{array}{l}3 . \\
04\end{array}$ & $\begin{array}{r}11 . \\
93\end{array}$ & $\begin{array}{r}1.8 \\
1\end{array}$ & 0.08 & 0.63 & 0.08 & 0.67 \\
\hline $\begin{array}{l}\text { BRIEF } \\
\text { Metacognition }\end{array}$ & $\begin{array}{r}64 \\
.2 \\
2 \\
\end{array}$ & $\begin{array}{r}12 \\
.1 \\
2 \\
\end{array}$ & $\begin{array}{r}62 . \\
83\end{array}$ & $\begin{array}{r}12 . \\
91\end{array}$ & 0.18 & 0.24 & 0.18 & 0.23 \\
\hline BRIEF Monitor & $\begin{array}{r}11 \\
.7 \\
2 \\
\end{array}$ & $\begin{array}{r}2 . \\
74\end{array}$ & $\begin{array}{r}11 . \\
66\end{array}$ & $\begin{array}{r}3.0 \\
2\end{array}$ & 0.04 & 0.78 & 0.04 & 0.85 \\
\hline $\begin{array}{l}\text { BRIEF } \\
\text { Organization of } \\
\text { Materials }\end{array}$ & $\begin{array}{r}11 \\
.3 \\
3 \\
\end{array}$ & $\begin{array}{r}2 . \\
79\end{array}$ & $\begin{array}{r}10 . \\
69\end{array}$ & $\begin{array}{r}3.3 \\
9\end{array}$ & 0.15 & 0.34 & 0.15 & 0.27 \\
\hline $\begin{array}{l}\text { BRIEF } \\
\text { Plan/Organize }\end{array}$ & $\begin{array}{r}34 \\
.2 \\
2\end{array}$ & $\begin{array}{r}23 \\
.5\end{array}$ & $\begin{array}{r}53 . \\
59\end{array}$ & $\begin{array}{r}23 . \\
74\end{array}$ & 0.06 & 0.71 & 0.06 & 1 \\
\hline
\end{tabular}




\begin{tabular}{l|r|r|r|r|r|r|r|r|r} 
BRIEF Shift & 9. & 2. & 10 & 2.1 & & -0.05 & 0.76 & -0.05 & 0.66 \\
\hline $\begin{array}{l}\text { BRIEF Working } \\
\text { Memory }\end{array}$ & 13 & 08 & 28 & 5 & 12. & 2.7 & & & \\
\hline
\end{tabular}

Table 7: Showing non-significant relationship between Male and Female estradiol level and EF Behavior subscales, A) Not Corrected and B) Corrected for age/gender, FDR corrected for multiple comparisons. Abbreviations: BRIEF, Behavior Rating Inventory of Executive Function; EF, executive function; FA, fractional anisotropy; pDLPFC, posterior dorsolateral prefrontal cortex; SA, surface area.

\section{Neuroimaging Results}

Next, we interrogated the BRIEF subscales, estradiol level, and their interaction, with cortical morphometry and FA. Of note, pubertal status showed no relationship with cortical morphometry or FA. The focus of this paper therefore, presents only estradiol level in relationship to EF, cortical morphometry and structural connectivity, as well as the impact of BRIEF subscale-by-estradiol interaction on brain measurements. The interaction between EF behavior and estradiol level is of particular interest; due to the fact previous studies suggest both variables have a close relationship to cortical morphometry and structural connectivity.

Given the possible effects of age/gender in the sample, an analysis investigating the unique role of age and/or gender on cortical morphometry was explored. The results indicate a negative correlation between age and the right superior frontal gyrus $[-\log (p)=-4.00, p=0.0001]$ and right superior temporal sulcus SA [- $\log (p)=-4.00, p=0.0001]$, suggesting higher age is related to less $S A$ in these regions. No relationship was observed between gender and cortical morphometry. 
Because the focus of the paper is concerned with EF behavior and estradiol level, age and gender are used as covariates in the remaining analyses.

Therefore, the following sections present morphometric and FA results in the following manner: relationships of EF with: cortical GM and b) FA (Table 7, Figure 20); relationships of estradiol level with: a) cortical GM and b) FA (Table 8, Figure 21); relationships of the interaction of EF and estradiol level_with: a) cortical GM and b) FA (Table 9, Figure 22). To be consistent throughout the results and discussion we refer to the positive relationship between EF behavior and estradiol level from the standpoint of higher BRIEF subscale scores (i.e., decreased EF behavior) and elevated estradiol level, to explain their effects on cortical morphometry and FA.

\section{Executive Function Results}

Cortical Morphometry

To examine relationships between cortical morphometry and EF (as measured by the BRIEF subscales), each subscale was regressed with surfacebased morphometry (SBM) measures (GMV, surface area and cortical thickness), controlling for age and gender. This analysis yielded a negative relationship between the Shift subscale and SA in the right pdIPFC $[-\log (p)=-2.19, p=0.006]$, indicating decreased ability shifting attention was related to less SA in the right pdIPFC (Figure 25A, Table 8). 


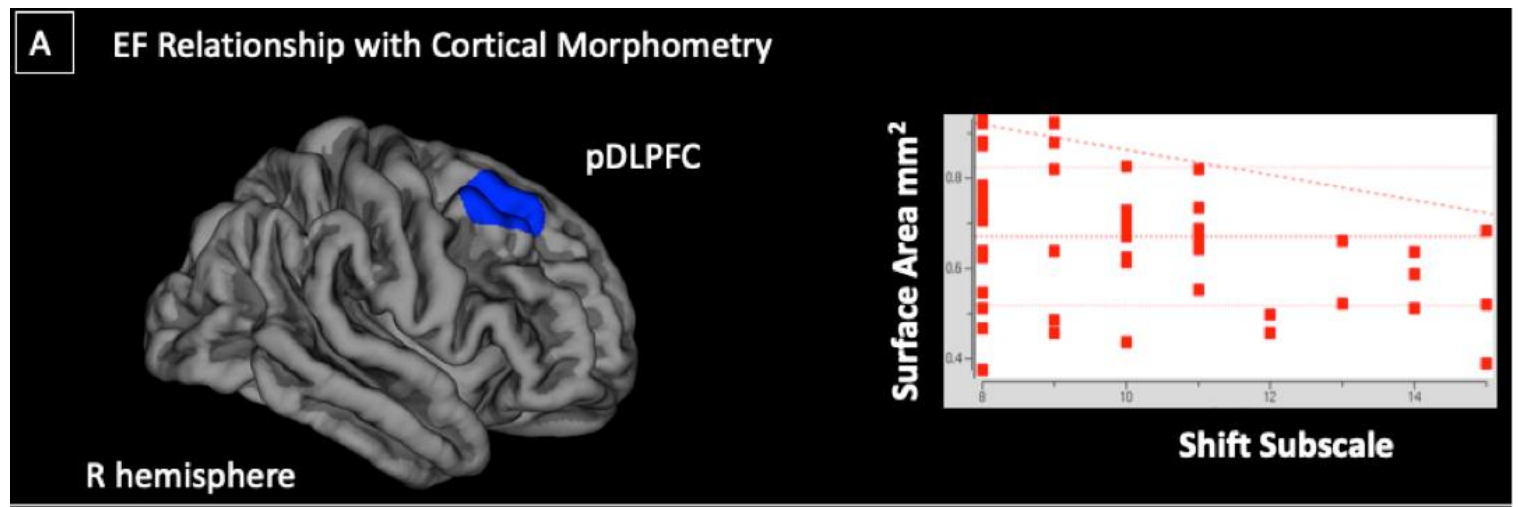

B EF Relationship with Structural Connectivity

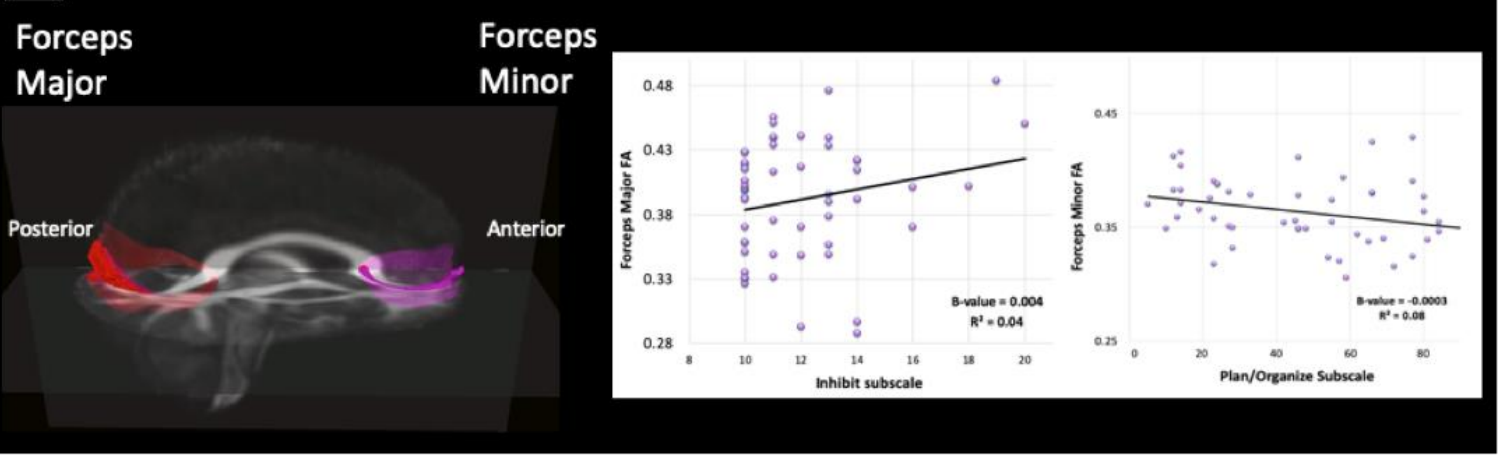

Figure 25: Executive function behavior relationship with cortical morphometry and structural connectivity. ( $N=51)$. (A) Significant negative relationship between surface area (SA) in the right pdIPFC and the BRIEF Shift subscale. (B) Significant relationships between two white matter tracts on a template atlas (forceps minor and major) with BRIEF Plan/Organize subscales and BRIEF Inhibit subscales, respectively.

\begin{tabular}{|l|l|l|l|l|}
\hline Measure & EF Behavior & Hemisphere & Directionality & $\begin{array}{l}\text { Region/T } \\
\text { ract }\end{array}$ \\
\hline SA & Shift & R & - & pdIPFC \\
\hline FA & Plan/Organize & None & - & $\begin{array}{l}\text { Forceps } \\
\text { Minor }\end{array}$ \\
\hline FA & Inhibit & None & + & $\begin{array}{l}\text { Forceps } \\
\text { Major }\end{array}$ \\
\hline
\end{tabular}

Table 8: Relationships of EF Behavior with cortical GM and FA. Abbreviations: $S A=$ surface area, $F A=$ fractional anisotropy, pdIPFC = posterior dorsolateral prefrontal cortex. EF, executive function; FA, fractional anisotropy; pdIPFC, posterior dorsolateral prefrontal cortex; $S A$, surface area. 


\section{White Matter Integrity}

Having examined grey matter relationships with EF behavior, we next investigated FA. The Plan/Organize subscale showed a negative relationship with FA of the forceps minor $\left(R^{2}=.07, p=0.01\right)$. Conversely, the Inhibit subscale showed a positive relationship with FA in the forceps major $\left(R^{2}=.04, p=0.02\right)$. These results indicated decreased ability putting order into play was associated with lower FA in the forceps minor, while decreased control over impulses was associated with higher FA in the forceps major (Figure 25B, Table 8).

\section{Estradiol Level Results}

Cortical Morphometry

No significant results were observed between cortical morphometry and estradiol level.

\section{White Matter Integrity}

Next, we examined the relationship between estradiol level and FA. A negative relationship between estradiol level and FA was observed in the right inferior fronto-occipital fasciculus (iFOF) $\left(\mathrm{R}^{2}=.09, p=0.01\right)$, indicating higher estradiol level related to lower FA in the right iFOF (Figure 26, Table 9). 


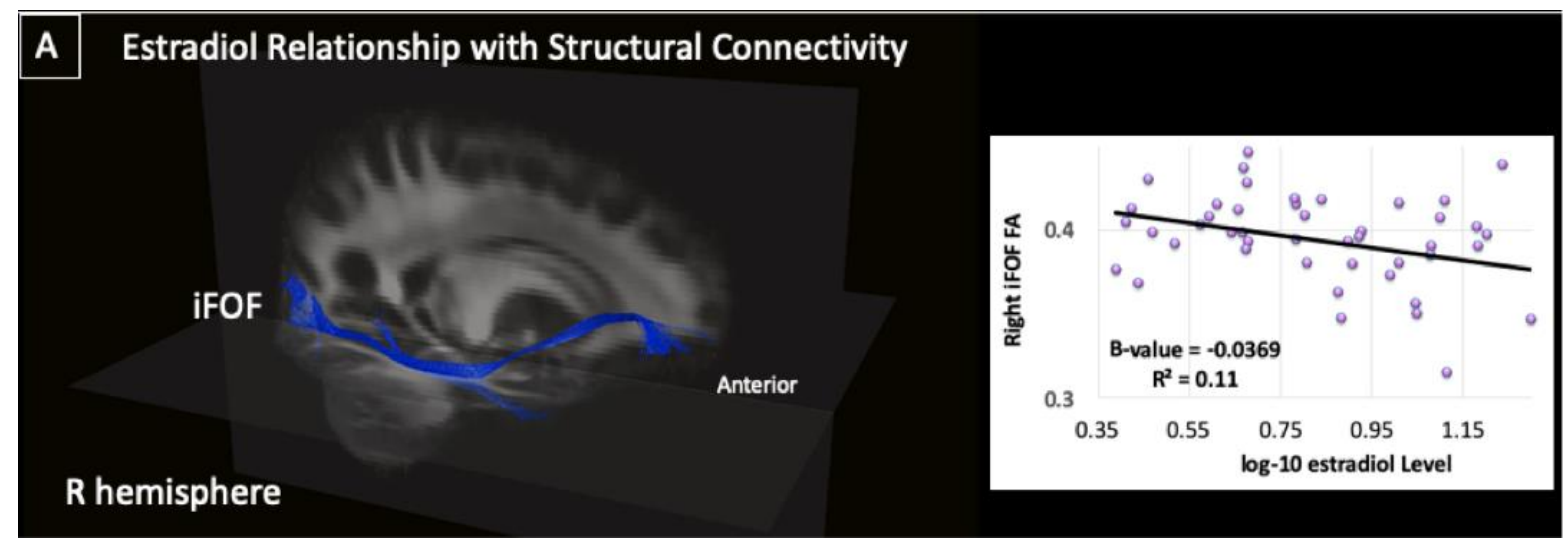

Figure 26: Estradiol relationship with structural connectivity $(N=51)$. (a) Significant negative relationship between right hemisphere iFOF fractional anisotropy (FA) and estradiol level on template atlas.

\begin{tabular}{|l|l|l|l|l|}
\hline Measure & Variable & $\begin{array}{l}\text { Hemispher } \\
\mathbf{e}\end{array}$ & Directionality & Region/Tract \\
\hline FA & Estradiol & $\mathrm{R}$ & - & $\mathrm{R}$ iFOF \\
\hline
\end{tabular}

Table 9: Relationship of estradiol level with FA. Abbreviations: FA = fractional anisotropy iFOF = inferior fronto-occipital fasciculus.

\section{Executive Function and Estradiol Interaction Results}

\section{Cortical Morphometry}

Because we hypothesized that estradiol level may interact with EF behavior, we investigated the BRIEF subscale-by-estradiol interaction and its effect on cortical morphometry. A negative relationship was observed between the Inhibitby-estradiol interaction and GMV in the right PMC and between the Working Memory-by-estradiol and GMV in the right PMC $[-\log (p)=-4.00, p=0.0001 ;-\log (p)$ $=-2.47, p=0.003$, respectively]. These results indicated that increased difficulty inhibiting one's actions and increased levels of estradiol related to less GMV in the right PMC. Additionally, increased difficulty holding information online and 
increased levels of estradiol related to less GMV in the right PMC (Figure 27A, Table 10).

Figure 27: BRIEF subscale and estradiol interaction relationships with cortical
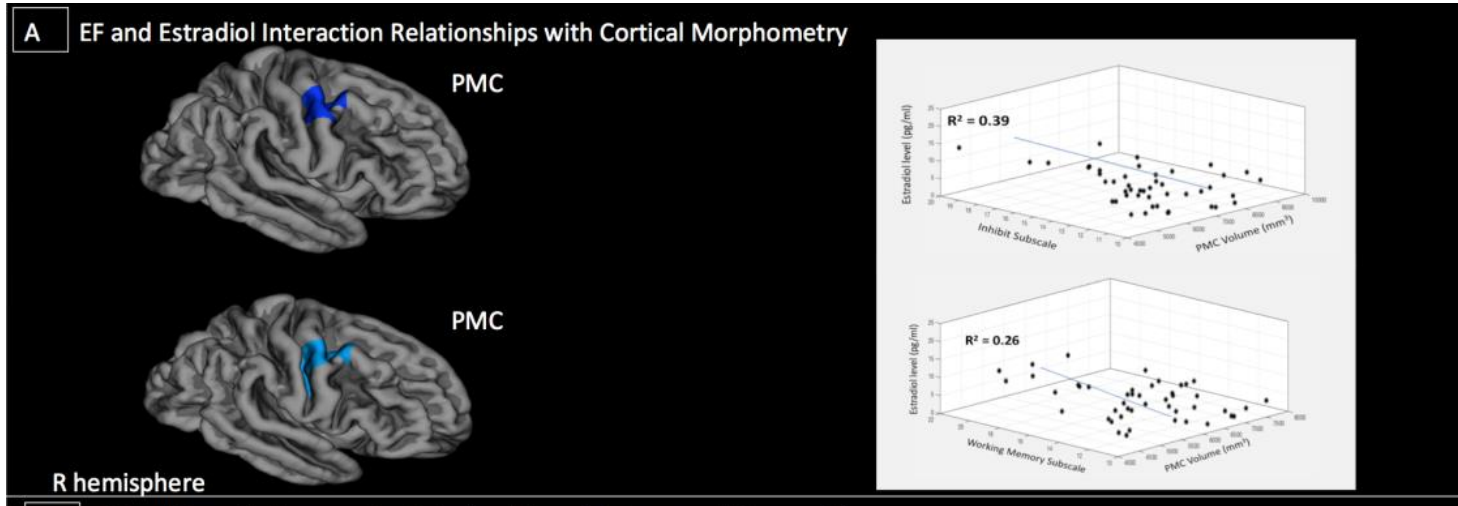

R hemisphere

B EF and Estradiol Interaction Relationships with Structural Connectivity

iFOF

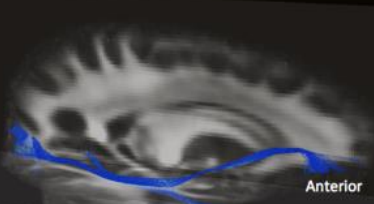

R hemisphere

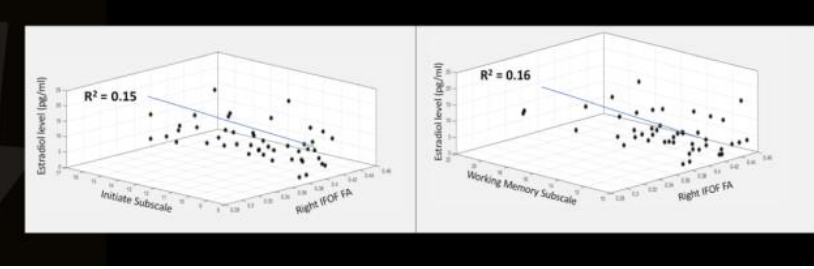

morphometry and structural connectivity. (A) Significant negative relationship between volume in the right PMC and the Inhibit-by-estradiol interaction and Working Memory-by-estradiol interaction. (B) Significant negative relationship between right iFOF FA and Initiate-by-estradiol and Working Memory-by-estradiol interactions on a template atlas

\begin{tabular}{|l|l|l|l|l|}
\hline $\begin{array}{l}\text { Meas } \\
\text { ure }\end{array}$ & $\begin{array}{l}\text { BRIEF subscale-by-estradiol } \\
\text { level }\end{array}$ & $\begin{array}{l}\text { Hemispher } \\
\text { e }\end{array}$ & $\begin{array}{l}\text { Directio } \\
\text { n }\end{array}$ & $\begin{array}{l}\text { Region/ } \\
\text { Tract }\end{array}$ \\
\hline $\begin{array}{l}\text { Volum } \\
\text { e }\end{array}$ & Inhibit-by-Estradiol & R & - & PMC \\
\hline $\begin{array}{l}\text { Volum } \\
\text { e }\end{array}$ & Working Memory-by-Estradiol & R & - & PMC \\
\hline FA & Initiate-by-Estradiol & R & - & R iFOF \\
\hline FA & Working Memory-by-Estradiol & R & - & R iFOF \\
\hline
\end{tabular}

Table 10. Relationships of BRIEF-by-estradiol subscales interaction with cortical GM and FA. Abbreviations: iFOF: inferior fronto-occipital fasciculus, PMC: primary motor cortex.

White matter integrity 
Finally, we examined the relationship between BRIEF subscales-byestradiol and FA interaction. The results showed negative relationships between the Initiate-by-estradiol interaction and FA in the right iFOF $\left(\mathrm{R}^{2}=0.15, p=0.01\right)$, and between the Working Memory-by-estradiol interaction and FA in the right iFOF $\left(R^{2}=0.16, p=0.008\right)$. The results suggested that increased estradiol level and decreased motivation of task initiation related to lower FA values in the right iFOF (Figure 27B, Table 10).

\section{Discussion}

Our results provide comprehensive evidence that individual differences in EF behavior and estradiol level, in a healthy adolescent sample, are linked to variation in aspects of cortical GM morphometry and FA of white matter tracts connecting the cerebral hemispheres and disparate anterior-posterior regions of the brain. Overall, decreased EF behavior related to decreased cortical grey matter morphometry and bidirectional white matter integrity, while increased estradiol level related to decreased white matter tract integrity. Lastly, increased magnitude of the interaction between EF behavior and estradiol level related to decreased cortical grey matter morphometry and white matter tract integrity. Below we discuss each finding and its relative implications.

Relationship between Executive Function and Estradiol Level

Firstly, we wanted to determine the relationship between estradiol level and EF behavior. We did not find any significant relationships between estradiol level 
and EF behavior, regardless of correcting or not correcting for age/gender. Previous evidence suggests that estradiol level is indeed related to EF (Hampson \& Morley, 2013; Hidalgo-Lopez \& Pletzer, 2017), albeit the results differ depending on the age range of the sample. It is possible our age range (7 to 18) did not have enough variability to produce statistically significant results.

Relationship between Executive Function and Cortical Morphometry

We next aimed to determine how EF behavior, aspects of cortical morphometry and FA are related. We first hypothesized decreased EF behavior should be associated with decreased aspects of cortical morphometry of the LPFC and decreased FA of tracts that support communication between prefrontal structures, as they have prominent roles in cognitive and emotional function. Our cortical morphometry results demonstrated decreases in EF behavior relating to moving freely from one activity to another, tolerating change and switching attention (Shift subscale) was associated with decreased SA in the right pdIPFC. Previous research indicates shifting, an EF feature imperative for changing one's own behavior according to environmental contexts, relies on the dIPFC (Karbach \& Unger, 2014; Ravizza \& Carter, 2008). Furthermore, a recent cortical morphometry study indicates that multi-task training leads to increases in right dIPFC GMV (Takeuchi et al., 2014), supporting the notion that shifting behavior depends on the dIPFC.

WM results indicated decreases in EF behavior relating to putting order into work and play (Plan/Organize subscale) was associated with decreased FA in the 
forceps minor. Research demonstrates the forceps minor is a fiber bundle which connects the lateral and medial surfaces of the frontal lobes and crosses the midline via the genu of the corpus callosum (Genova et al., 2013). When damaged by disease, the forceps minor is linked to robustly diminished processing speed and cognitive impairment, indicating its interhemispheric connections between the PFC contribute to EF (Biesbroek et al., 2016; Genova et al., 2013). The association between damage to these tracts and reduced performance in the Trail-Making task has been reported in schizophrenia (Pérez-Iglesias et al., 2010) and traumatic brain injury (Kraus et al., 2007). Previous studies in Multiple Sclerosis (MS) have also noted a correlation between reduced FA in the forceps minor and Paced Auditory Serial Addition Test (PASAT) performance (Hecke et al., 2010). Our results therefore echo previous findings: decreased EF behavior is related with decreased FA of the forceps minor.

Conversely, decreased EF behavior related to controlling impulses (Inhibit subscale) was associated with increased FA in another WM tract, the forceps major. The forceps major is a is a fiber bundle which connects the occipital lobes and crosses the midline via the splenium of the corpus callosum (Prasad et al., 2015), and is thought to aid visuo-spatial function (Tamura et al., 2007). Lesions of the forceps major are associated with deficits in multi-tasking (Burgess et al., 2000), allocation of attentional resources and other information processing requiring integrated hemispheric function (Rossi et al., 2012). An increase of FA in the forceps major suggests efficient and speedy processing of incoming visuo- 
spatial material and thus may result in difficulties inhibiting behavior. Indeed, patients with conditions posited to arise from axonal overconnectivity such as autism spectrum disorder (ASD), attention deficit hyperactivity disorder (ADHD) and schizophrenia exhibit reduced inhibitory control (Solso et al., 2016; Tamm et al., 2012; R. Taylor et al., 2016). Thus, our findings suggest that FA changes in the forceps major affect attention-based cognitive functions such as impulse control and highlight the complex relationship between white matter structure and EF behavior.

Relationship between Executive Function, Cortical Morphometry and Structural Connectivity

We next investigated whether estradiol level had any relationship to cortical morphometry and FA. We next hypothesized decreased cortical morphometry in the LPFC and reduced FA, as studies indicate that decreased cortical morphometry and FA may both be related to increases in estradiol level in adolescent individuals (Herting et al., 2012; Peper et al., 2011; Witte et al., 2010). Contrary to our hypothesis, we found no such relationship with cortical morphometry. However, our results indicated that increased estradiol level related to decreased FA of the right iFOF. The iFOF, a long association WM bundle connects the inferior and lateral regions of the PFC through the inferior temporal lobes, terminating in lateral occipital regions (Ashtari, 2011). Research indicates the iFOF plays a critical role in attention and visual processing (Catani \& Thiebaut de Schotten, 2008; Yupeng Wu et al., 2016). Our findings mirror results indicating 
significant differences in long-range association fibers including the iFOF during adolescence when relationships with estradiol level were considered (Herting et al., 2012). The present study's results point to a relationship between estradiol level and this important WM tract connecting anterior-posterior regions of the cortex which may underlie EF behavior.

Relationship between Executive Function, Cortical Morphometry, Structural Connectivity and Estradiol

The final aim of the study was to determine the relationship between the interaction between BRIEF subscales and estradiol (i.e., BRIEF subscale-byEstradiol) with cortical GM and FA. At last, we hypothesized that decreased EF behavior would be related to increased levels of estradiol, which may consequently relate to reductions in aspects of cortical morphometry and structural connectivity. Our cortical morphometry findings indicated that decreased EF behavior related to controlling impulses (Inhibit subscale) and holding information online (Working Memory Subscale) coupled with increased estradiol level was associated with less GMV in the PMC. Studies indicate extensive connections exist from the anterior PFC to PMC (Fregni et al., 2005), which are thought to coordinate the integration of higher level EF processes and motor planning in service of goal attainment. Moreover, research demonstrates the LPFC has an increased number of estradiol receptors (Almey et al., 2015) which may result in increased sensitivity of estradiol in this region posited to underlie EF processes. Indeed, estradiol's impact on working memory is well documented, with high levels of estradiol impairing LPFC- 
dependent working memory, while low level estradiol weakly facilitating it (Bimonte \& Denenberg, 1999; Holmes et al., 2002; Wide et al., 2004). Therefore, our results append to existing findings, suggesting that changes in cortical morphometry may reflect more complex interactions between EF behavior and estradiol level affecting the LPFC.

Finally, our study's WM analyses suggested more difficulties with EF behavior related to beginning an activity (Initiate subscale), holding information online when completing a task (Working Memory subscale) and elevated estradiol level were associated with lower FA in the right iFOF. Previous research indicates that elevated estradiol level is related to decreases in EF behavior in adolescents (Lenroot \& Giedd, 2010; Peper et al., 2009, 2011) and that FA in the iFOF may be an important neural correlate of EF (Santiago et al., 2015). Thus, our results suggest an important relationship between the interaction of EF behavior and estradiol level on FA in the iFOF, a WM tract providing communication between disparate anterior-posterior brain regions, putatively underlying EF behavior.

\section{Limitations and Future Directions}

The study had several limitations. First, the study design was crosssectional and not longitudinal, which prevented depiction of individual trajectories, differences in change and direct estimation of relationships between change across different morphometric measurements. The conclusions from the present study should be replicated in longitudinal studies. Although a longitudinal approach has many merits, because multimodal imaging and hormonal data was only 
available for a large enough sample during one visit per participant, our study's aims were only possible with a cross-sectional approach. Second, although pubertal status was taken into account (using the Tanner Stage), menstrual cycle data was not recorded for the female participants, which could further result in fluctuations in estradiol level across the cycle and affect EF behavior. Thirdly, since the BRIEF subscales are highly intercorrelated, discerning their individual impact on brain morphometry is difficult, but speak to their contribution to EF as a whole. Fourthly, no other hormones related to the menstrual cycle were collected. Literature suggests that during the menstrual cycle, both estradiol and progesterone levels fluctuate rapidly, and a difference of a few hours can matter dramatically for estradiol levels. Rapidly changing effects of this hormone, coupled with age differences, suggest that these are important factors to keep in mind when researching the effects of cycling in females. Lastly, the measurement of estradiol level from saliva has drawbacks, especially in an adolescent population. Although great care was taken to understand the relationship between estradiol level, EF behavior and aspects of cortical morphometry and structural connectivity, saliva measurements are greatly affected by the use of exogenous hormones such as birth control or transdermal creams (Lewis et al., 2002). Furthermore, results should be interpreted cautiously due to lack of contraceptive and menstrual cycle data.

Future studies should continue to combine EF behavior (such as the BRIEF subscales), estradiol level and multi-modal neuroimaging methods in order to 
disentangle the function-estradiol-structure relationship in this critical neurodevelopmental period in cortical morphometry and structural connectivity thought to underlie EF processes. Specifically, the roles of peptide hormones like oxytocin and vasopressin should be investigated in the neural development of the adolescent brain and its relationship to EF processes.

\section{Summary}

To our knowledge, this is the only study investigating how individual differences in EF behavior and estradiol level relate to aspects of cortical morphometry and FA in a healthy, adolescent population. Our study implies that decreased EF behavior and elevated estradiol level relate to decreased aspects cortical morphometry and FA. Specifically, EF behavior and its interaction with estradiol level related to decreases in aspects of cortical morphometry in the pdIPFC, comprising the LPFC, an area well known to subserve goal directed behavior (Asplund et al., 2010; Yamagata et al., 2012). Further, EF behavior and its interaction with estradiol level were associated with bidirectional differences of FA measurements in interhemispheric connections (forceps minor and major, respectively) and long-range association fibers (iFOF) connecting anteriorposterior regions of the cortex. Thus, the results imply that variation in EF behavior and estradiol level relate to WM tracts supporting communication between cortical regions. 


\section{EXPERIMENT 5:}

Gender Differences in Functional Connectivity during Emotion Regulation

Beliefs in gender differences in emotion pervade our culture, with the striking stereotype being that women are more emotionally responsive to negative stimuli than men. While some empirical evidence mirrors the anecdotal conception (Fabes \& Martin, 1991; Fischer, 1993; Grossman \& Wood, 1993; Hess et al., 2000; Plant et al., 2000; Timmers et al., 2003), some researchers (Garnefski et al., 2004; Nolen-Hoeksema, 2001; Thayer et al., 1994) conjecture the basis for differences in emotion arise due to variations in emotion regulation (ER). Yet, despite the increasing recognition that deficient $E R$ is at the core of various affective disorders (Berking et al., 2014; Dalili et al., 2015; Green et al., 2007; Joormann \& Stanton, 2016; O'Driscoll et al., 2014; M. L. Phillips et al., 2008), and importantly, that women show an increased prevalence for these conditions (Kessler et al., 1993; Leach et al., 2008; Nolen-Hoeksema, 2001, 2012; Thomsen et al., 2005), the neural basis of gender differences in ER remains poorly understood.

ER describes processes that individuals use to influence the experience and expression of emotions (Giombini, 2015; James J. Gross, 2015; Ochsner \& Gross, 2005). ER is a complex process that includes the initiation, inhibition, or modulation of internal emotional states and emotion-related cognitions, physiological processes and behaviors (Compare et al., 2014). Importantly, an individual's ability to manage emotional experience and adapt to changing 
environments is crucial for mental health (James J. Gross \& Muñoz, 1995). The most prominent neurobiological ER model focuses on the modulatory effect of the prefrontal and cingulate regions involved in top-down control over the affective instantiation regions as a function of one's regulatory goal, tactic, nature of stimuli and emotions being regulated (Ochsner \& Gross, 2005; Ochsner et al., 2012). Specifically, brain regions implicated in top-down control include the dorso and ventrolateral, and dorso and ventromedial prefrontal cortex (dIPFC, vIPFC, dmPFC, vmPFC), orbitofrontal cortex (OFC), inferior parietal cortex (IPC) and anterior cingulate cortex (ACC), while those underlying emotion generation comprise the insulae, amygdalae and basal ganglia (Ochsner \& Gross, 2005; Ochsner et al., 2012). Within this model however, few (and equivocal) findings have emerged from functional neuroimaging studies investigating gender differences. For example, McRae et al., 2008 showed lower increases in prefrontal activity (ACC, IPC) and greater decreases in amygdala activity during ER efforts in men compared to women, despite no difference in self-reported negative emotion between genders. Domes et al., 2010 found the opposite activation pattern, indicating greater prefrontal activity (vmPFC) in men compared to women during employment of ER, with no notable gender differences in amygdala activity or self-report regulation success. Interestingly, both studies indicate a more efficient ER process in men, suggesting less effortful cognitive control (McRae et al., 2008) and more precise recruitment of areas putatively underlying ER (Domes et al., 2010) in men compared to women. In parallel, Mak 
et al., 2009's study argues that while men tend to utilize brain regions underlying cognitive control (lateral PFC and ACC) to a greater extent than women during ER, women tend to use emotion-associated ones (medial OFC), with comparable self-reported regulation success. In an effort to synthesize this contradictory information, a recent meta-analysis assembling the aforementioned studies proposes men may be more efficient (i.e., use less effortful cognitive control) in ER as evidenced by greater increases of fronto-parieto-temporal activity, and greater decreases of limbic/subcortical activity, while women may experience emotions with greater frequency and intensity as evidenced by increased activity in limbic regions (Whittle et al., 2011).

While some information regarding gender differences in ER may be gleaned from functional activity studies, connectivity within ER-related brain regions may provide another avenue for understanding how men and women differ in regulatory mechanisms. Our previous work has detailed righthemispheric functional pathways responsible for inhibitory regulation of emotional reactivity between the right anterior middle frontal gyrus (aMFG) and the OFC, and between the right OFC with the amygdala (Depue et al., 2016). Previously, we proposed this network may function hierarchically, with higher-order maintenance and updating of task goals performed by the right aMFG, and modulation of the amygdala (downstream effectors) implemented by an intermediary region (OFC) that exhibits direct anatomical connectivity. Similar results were found by Banks et al., 2007, a study that showed individuals who 
are more successful in regulating their emotion demonstrate greater effective connectivity (EC, a measure of connectivity relative to the task) between amygdala and lateral and medial PFC. Yet presently, scant functional connectivity (FC) data between these regions exists that examines gender differences related to ER. One such resting-state study found FC within the centromedial amygdala displayed gender-specific variations in association with trait-level ER (Yan Wu, Li, et al., 2016). The authors suggest connectivity patterns and higher ER (as determined by a self-reported behavioral measure) in this sub-region of the amygdala in women was linked to internal and emotional focus, while men's connectivity pattern and higher ER related to a greater ability to downregulate negative emotion compared to women. Lastly, one study investigating gender differences in EC during negative emotion processing demonstrated EC from the right amygdala to the dmPFC is significantly stronger in men compared to women, with the authors concluding that men may have a more evaluative rather than purely affective, in-the-moment, brain response during negative emotion processing (Lungu et al., 2015). To the best of our knowledge, however, no study to date has utilized a network-based approach to examine gender differences in FC during an ER task.

\section{Aim}

The present $\mathrm{fMRI}$ study investigated whether FC between brain regions putatively underlying ER differed between men and women during an ER task. 


\section{Hypotheses}

Based on prior studies investigating gender differences during $E R$, we have three hypotheses: (1) women will rate negative stimuli as more distressing, as compared to men; (2) women will show increased connectivity between brain regions putatively underlying emotional response (amygdala, hippocampus), as compared to men; (3) men will show increased connectivity between regions underlying top-down control of emotion (parietal cortex, dIPFC, VLPFC), as compared to women.

\section{Methods}

\section{Participants and Procedure}

A total of 55 healthy young adults without a reported history of neurological or psychiatric disorders were recruited for this study. Seven participants were excluded from analyses due to incomplete behavioral data $(n=1)$, various scanning issues resulting in incomplete fMRI data $(n=4)$, and disclosed psychiatric diagnosis following consent $(n=2)$. This left 48 (20 women) participants in the final sample. Men and women were comparable in age and years of education $(t(46)=-3.99, p=0.69$ and $t(46)=-.15, p=0.88$, respectively). fMRI Task - Emotion Regulation Task (ERT)

The ERT was divided into three parts: (A) ER Baseline (B) Emotion Regulation (ER) (C) ER Rating. Only parts A \& B were BOLD scans, part C was structural. 


\section{(A) ER Baseline:}

The ER Baseline task employed a hybrid event-related design that contained mini-blocks presented in pseudorandom order. 20 negative International Affective

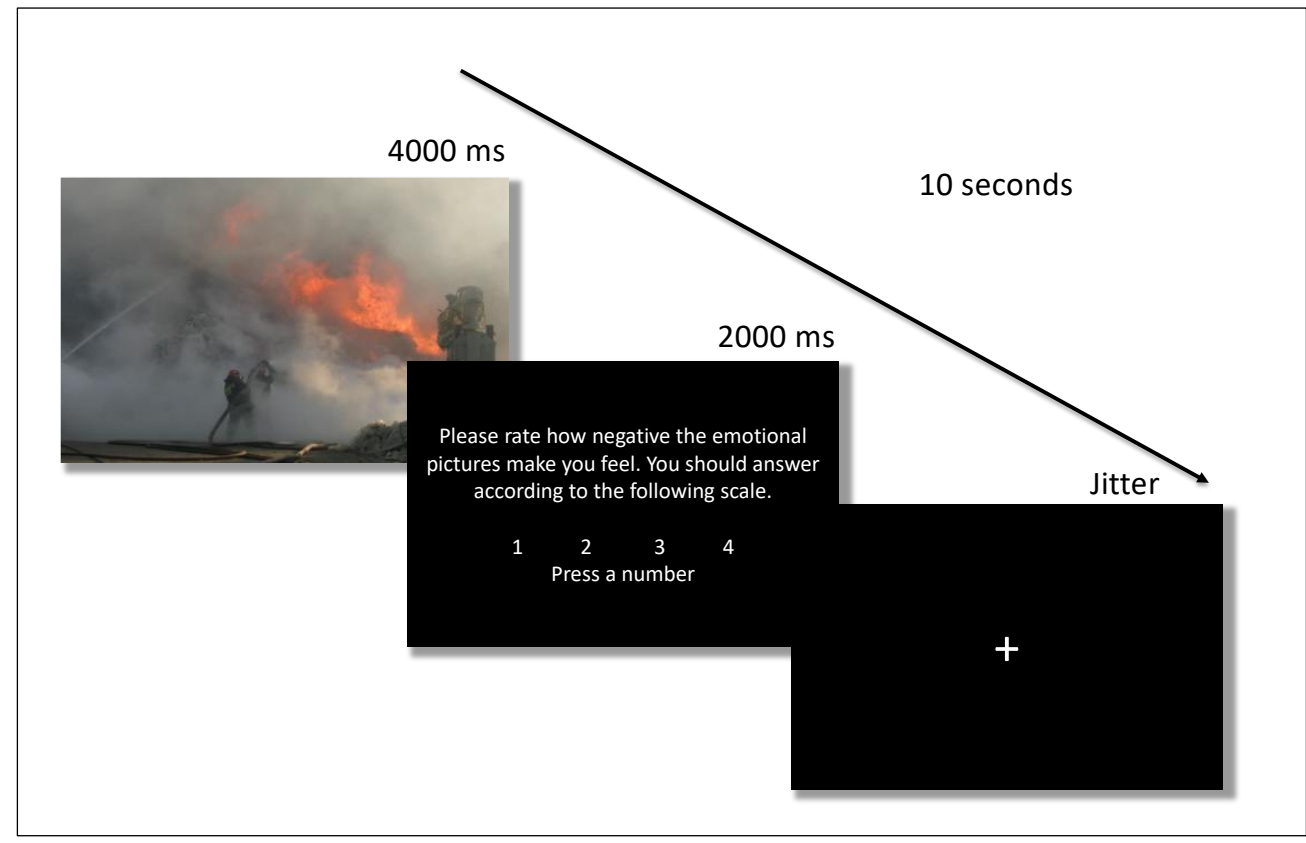

Picture System (IAPS) pictures and 10 neutral pictures were displayed (Lang et al., 2005). The pictures were displayed for 4 seconds. This design was chosen to balance considerations for the psychological state of the participant with statistical power. Following presentation of each stimulus, participants rated the image for how negative it made them feel, using a 4-point Likert scale $(1=$ None to 4 = Extremely negative; 2 seconds to rate; Figure 28). This allowed acquisition of a subjective negative baseline rating for each participant. A pseudorandom variable jittered ITI was incorporated to increase design efficiency for hemodynamic response estimation $(0-10 \mathrm{sec})$. Resultant behavioral ratings from the task were further used to calculate ER Suppression Score (see part C). 
Figure 28: Example of a single trial from ER Baseline Task (Image displayed not part of actual stimuli set).

(B) Emotion Regulation (ER):

The ER task also employed a hybrid event-related design with mini blocks presented in pseudorandom order, whereby a different set of negative and neutral pictures selected from the IAPS database were displayed (Lang et al., 2005). Neutral and negative stimuli valence $(M=5.68, S D=0.71 ; M=2.97$, $S D=0.61$, respectively) and arousal $(M=4.82, S D=0.62 ; M=5.27, S D=0.39$, respectively) values both were statistically different between the two sets $t(78)=$ $18.35, p<.01 ; t(78)=-3.84, p<.01$, respectively. First, the words 'SUPPRESS' in the color red or 'VIEW' in the color blue appeared as cues for $500 \mathrm{~ms}$ to prepare the participant for the upcoming picture. Next, negative pictures surrounded by a red border (Suppress trials) and neutral pictures surrounded by a blue border (View trials) were presented for 3.5 seconds (Suppress trials $n=30$, View trials $n$ = 12) (Figure 29). The participant was instructed to “'decrease or detach' from the emotion when the border was red, and 'simply view' the picture when the border was blue". The "Suppress" instructions were worded simply in order to encourage the participant to use their default ER method. A pseudorandom variable jittered ITI was incorporated to increase design efficiency for hemodynamic response estimation $(0-4 \mathrm{sec})$. The pictures repeated once, with a 60 second break in-between. To test the successful induction of negative emotion during the ER Task, a two-tailed paired samples $t$-test was conducted 
between mean ratings of the $\operatorname{View}(M=1.3, S D=0.52)$ and Suppress $(M=2.5$, $S D=0.52)$ pictures, which revealed a significant difference between the two conditions $t(47)=-10.59, p<0.01$.

Figure 29: Example of a single trial of each condition from Emotion Regulation (ER) (Image displayed not part of actual stimuli set).

\section{(C) ER Rating}

During a structural scan, the same images used in part B were then displayed without any border for 4 seconds. Following presentation of each image,

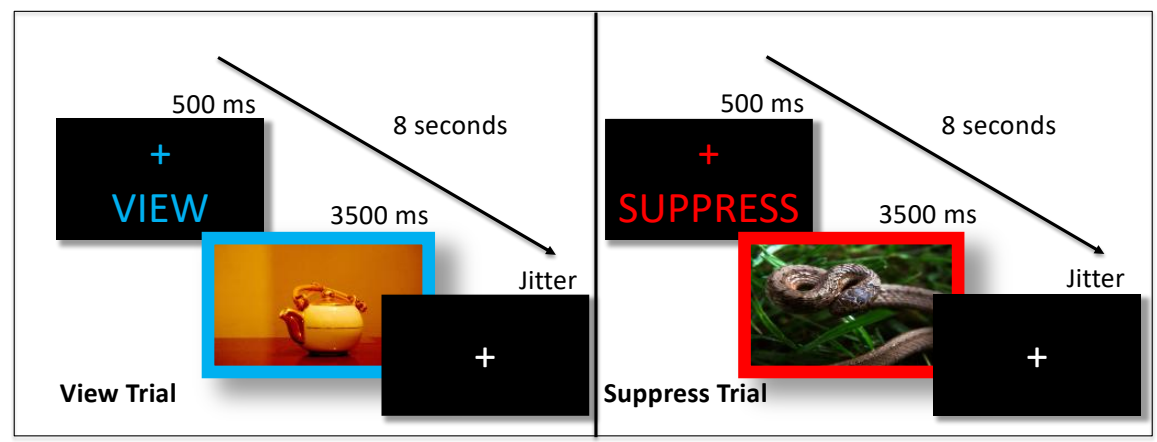

participants rated how negative the image made them feel, using a four-point Likert scale $(1=$ None to $4=$ Extremely negative, displayed in the same way as ER Baseline). To test whether the participants were successfully able to downregulate their emotional response to the images, a two-tailed paired samples $t$ test was conducted between the negative Baseline Ratings ( $M=2.7, S D=0.50)$ 
and $E R$ negative ratings $(M=2.4, S D=0.52)$, showing a significant difference in scores $t(46)=5.4, p<0.01$. Furthermore, the negative ER ratings from this part were subtracted from the earlier acquired negative baseline ratings (part A) in order to calculate a Suppression Score. The Suppression Score was derived from negative ratings only (rather than ratings on "View" trials) to ensure an accurate measurement of negative affect down-regulation success (i.e. comparing a response to negative stimuli before and after ER). The Suppression Score was used as an independent variable in subsequent FC analyses which served as the dependent variable.

\section{Self-Report ER Strategy Questionnaire}

Immediately after the scan, participants exited the scanner and were asked to view the ER task (part B) pictures on a computer screen. Each photo was followed by the instructions "type any strategies you may have used while downregulating your emotion on the following screen." The resultant statements were coded and summed by two independent raters and categorized as: "Cognitive Reappraisal" if they were characterized by reframing the emotional situation (ex. "I tried not to think negatively, but to think positive") (Ochsner \& Gross, 2005), Attentional Control if they were characterized by volitionally diverting attention to something unrelated (ex. "I focused on my breathing and tried be analytical about the wound") (Webb et al., 2012), and Other if it was characterized by another ER strategy ("It looks like stock photo, no real threat"). Data for 4 participants was 
not available due to technical errors, and 2 subjects were removed for misunderstanding task instructions, leaving $\mathrm{N}=43$ for analysis.

Imaging Data Acquisition

\section{Functional}

Functional blood oxygenation level-dependent (BOLD) images were collected using gradient-echo T2*-weighted echoplanar imaging $(T E=28 \mathrm{~ms} ; \mathrm{TR}=2000$ $\mathrm{ms}$; flip angle $=79^{\circ} ; \mathrm{FoV}=204 \mathrm{~mm}$; voxel size $=3.2 \mathrm{~mm}^{3}, 38$ interleaved slices). Slices were oriented obliquely along the AC-PC line. Imaging Data Analysis

All analyses and visualizations were conducted using the CONN toolbox 18.b (Whitfield-Gabrieli \& Nieto-Castanon, 2012) based on SPM12 (Penny et al., 2007) in the 2017 version of MATLAB. Spatial preprocessing in the CONN toolbox included realignment, normalization and smoothing (8 $\mathrm{mm}$ FWHM Gaussian filter) using SPM12 default parameter settings. Anatomical volumes were segmented into gray matter, white matter and cerebrospinal fluid (CSF) areas, and the resulting masks were eroded to minimize partial volume effects. The temporal time series characterizing the estimated subject motion, as well as the blood-oxygen-level dependent (BOLD) fMRI time series within the subjectspecific white matter mask (five principal component analysis (PCA) parameters) and the CSF mask (five PCA parameters), were used as temporal covariates and removed from the BOLD functional data using linear regression. Stimuli onsets and duration were specified in the toolbox, so that BOLD time series could be 
appropriately divided into task-specific mini blocks. Single trial regressors for the main effects were constructed by modeling each of the conditions versus lowlevel fMRI baseline. For ER Baseline: Negative, Neutral; for ER Task: View, Suppress. The contrasts of interest were created by comparing conditions against one another: For ER Baseline, Negative>Neutral, Neutral> Negative and for ER Task, View > Suppress and Suppress $>$ View. For each regressor, a double-gamma hemodynamic response function (HRF) was convolved with an event vector starting at the cue onset through stimulus presentation (baseline duration of $6 \mathrm{sec}$ + variable jittered ITI, and for ER Task - $4 \mathrm{sec}$ + variable jittered ITI). This data was subsequently temporally filtered and the resulting residual BOLD time series were then band-pass filtered $(0.008-$ inf $\mathrm{Hz})$, as this filter benefits from keeping higher-frequency information fitting event-related tasks. $A$ region of interest $(\mathrm{ROI})$ based correlation approach was used to evaluate temporally correlated BOLD signal between 28 a priori selected $10 \mathrm{~mm} \mathrm{ROI}$ (sphere around provided coordinates with $10 \mathrm{~mm}$ radius) from CONN's default atlas which combines FSL's Harvard-Oxford atlas and FOX's ROIs. The selection of these ROls was based on previous research demonstrating their involvement in ER (Depue et al., 2016; Kohn et al., 2014; Picó-Pérez et al., 2017). They included: bilateral - ventral frontal pole (FP), orbital frontal cortex (OFC), IFG operculum (IFG oper), middle frontal gyrus (MidFG), inferior frontal gyrus (IFG, pars opercularis), inferior frontal gyrus (IFG, pars triangularis), posterior supramarginal gyrus (pSMG), angular gyrus (AG), anterior parahippocampal 
gyrus (aPaHC), posterior parahippocampal gyrus ( $\mathrm{pPaHC})$, hippocampus, amygdala, and the subcallosal Cortex (SubCalc), dorsal anterior cingulate gyrus (dACC) (Table 11).

\section{Table 11}

Selected Regions of Interest (ROIs) for Connectivity Matrices

\begin{tabular}{|c|c|c|c|}
\hline \multirow[t]{2}{*}{ ROI Abbreviation } & \multirow[t]{2}{*}{ ROI Name } & \multicolumn{2}{|c|}{ MNI Coordinates } \\
\hline & & $\mathrm{X}$ & $\bar{Z}$ \\
\hline R FP & R Ventral Frontal Pole & 26.1552 .14 & 8.25 \\
\hline & & - & \\
\hline LFP & L Ventral Frontal Pole & 24.7252 .95 & 7.5 \\
\hline R OFC & R Orbital Frontal Cortex & 29.1123 .07 & -16.23 \\
\hline & & - & \\
\hline L OFC & L Orbital Frontal Cortex & 29.5423 .66 & -16.57 \\
\hline R IFG Oper & R IFG, Operculum Cortex & 41.1118 .62 & 4.91 \\
\hline L IFG Oper & L IFG, Operculum Cortex & -39.718 .32 & 4.52 \\
\hline R MidFG & R Middle Frontal Gyrus & 39.1118 .62 & 42.78 \\
\hline
\end{tabular}

$\begin{array}{llll}\text { L MidFG } & \text { L Middle Frontal Gyrus } & 38.0718 .43 & 42.06 \\ \text { R IFG tri } & \text { R IFG, pars triangularis } & 51.8627 .76 & 7.7 \\ & & - & \\ & & 49.7128 .49 & 8.66 \\ \text { L IFG tri } & \text { L IFG, pars triangularis } & & \\ \text { R IFG oper } & \text { R IFG, pars opercularis } & 52.2115 .41 & 16.2\end{array}$




\begin{tabular}{|c|c|c|c|}
\hline \multirow[t]{2}{*}{ L IFG oper } & L IFG, pars opercularis & \multirow[t]{2}{*}{50.6414 .51} & \multirow[t]{2}{*}{15.39} \\
\hline & R Supramarginal Gyrus, & & \\
\hline \multirow[t]{2}{*}{ R pSMG } & posterior division & $55.2-40.36$ & 33.6 \\
\hline & L Supramarginal Gyrus, & - & \\
\hline L pSMG & posterior division & $54.88-46.02$ & 33.24 \\
\hline \multirow[t]{2}{*}{ R AG } & R Angular Gyrus & $51.93-51.8$ & 32.35 \\
\hline & & - & \\
\hline \multirow[t]{2}{*}{ L AG } & L Angular Gyrus & $50.35-55.7$ & 29.76 \\
\hline & Dorsal Anterior Cingulate & & \\
\hline \multirow[t]{2}{*}{$\mathrm{dACC}$} & Gyrus & $0.8 \quad 18.29$ & 24.34 \\
\hline & R Anterior Dorsal Cingulate & & \\
\hline \multirow[t]{2}{*}{$\mathrm{RdACC}$} & Gyrus & 6.5536 .56 & 22.69 \\
\hline & L Anterior Dorsal Cingulate & & \\
\hline \multirow[t]{2}{*}{ LdACC } & Gyrus & -6.236 .65 & 20.78 \\
\hline & R Parahippocampal Gyrus, & & \\
\hline \multirow[t]{2}{*}{$\mathrm{R}$ aPaHC } & anterior division & $22.35-8.05$ & -30.25 \\
\hline & L ParahippocampalGyrus, & - & \\
\hline \multirow[t]{2}{*}{$\mathrm{LaPaHC}$} & anterior division & $21.86-9.1$ & -30.3 \\
\hline & R Parahippocampal Gyrus, & & \\
\hline $\mathrm{R} p \mathrm{paHC}$ & posterior division & $22.9-30.53$ & -16.75 \\
\hline
\end{tabular}


L Parahippocampal Gyrus, -

$\begin{array}{lll}\text { L pPaHC } & \text { posterior division } & 21.89-32.42-16.88 \\ \text { R Hip } & \text { R Hippocampus } & 26.49-20.95-14.25\end{array}$

L Hip L Hippocampus 25.17-23.19-13.8

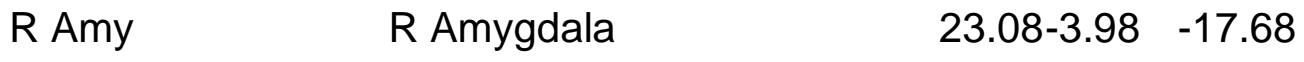

L Amy L Amygdala $\quad 22.99-4.94 \quad-17.73$

$\begin{array}{llll}\text { SubCalc } & \text { SubcallosalCortex } & -0.0720 .53 & -14.83\end{array}$

The CONN toolbox was used to conduct an ROI-to-ROI analysis, by grouping voxels into ROls based upon CONN's default atlas. Each ROI value represents a mean functional connectivity estimate within that particular ROI. Connectivity values were computed as the Fisher-transformed bivariate correlation coefficient between a pair of ROI BOLD time series, where a positive correlation indicates positive FC and a negative correlation indicates negative FC between ROls. To explore the effects of the different conditions (ER Baseline: Negative, Negative vs. Neutral; ER Task: Suppress, Suppress vs. View) and the two groups (men and women), Wilks' lambda or F-statistics were used to illustrate main effects of each group together with the main and cross effects of the conditions. Effect sizes for connectivity contrasts between all ROI sources were calculated alongside $T$, and $F$ values; and false discovery rate-corrected (FDR) $p$-values 
were calculated for each specified second-level analysis. The T-stat represents the results of between-subjects conditions contrasts when testing each individual connection separately between each pair of seed and target ROls, while the Fstat represents the same results but testing each individual seed region separately and looking for any effects across all target ROls. We were interested which particular 28 a priori ROls show differences in FC between genders, therefore we report T-stats and FDR-corrected ( $p$-value of 0.05 ) (seed-level) results below. The CONN toolbox ROI-to-ROI analyses results are considered appropriately corrected for multiple comparisons across all brain and analysis voxels when the voxel-level and the extent cluster-level thresholds use an analysis-wise false positive control FDR-corrected p-values method (WhitfieldGabrieli \& Nieto-Castanon, 2012).

Results

\section{Behavioral Results}

To examine subjective emotion ratings prior to emotion regulation (ER Baseline; part A), we conducted an independent samples $t$-test comparing the means of self-reported negative affect between genders (based on 5000 bootstrap samples). Due to our small sample size, we performed the bootstrapped t-test to guarantee a good approximation of population measurements and conducted Levene's test for homogeneity of variances due to unequal sample sizes. The results showed a statistically significant difference between men's $(M=2.57$, 
$S D=0.54)$ and women's $(M=2.99, S D=0.33)$ negative ER Baseline ratings, $t(45)=-$

3.04, $p=0.004,95 \% \mathrm{Cl}[-0.70$ to -0.14$]$. Levene's test indicated unequal

variances $(F=5.40, p=.03)$, so degrees of freedom were adjusted from 45 to 44.6 .

No gender differences were observed between neutral ER baseline ratings

$t(45)=-1.35, p=0.18,95 \% \mathrm{Cl}[-0.21$ to 0.04$]$ (Figure 30).

\section{ER Baseline Ratings}

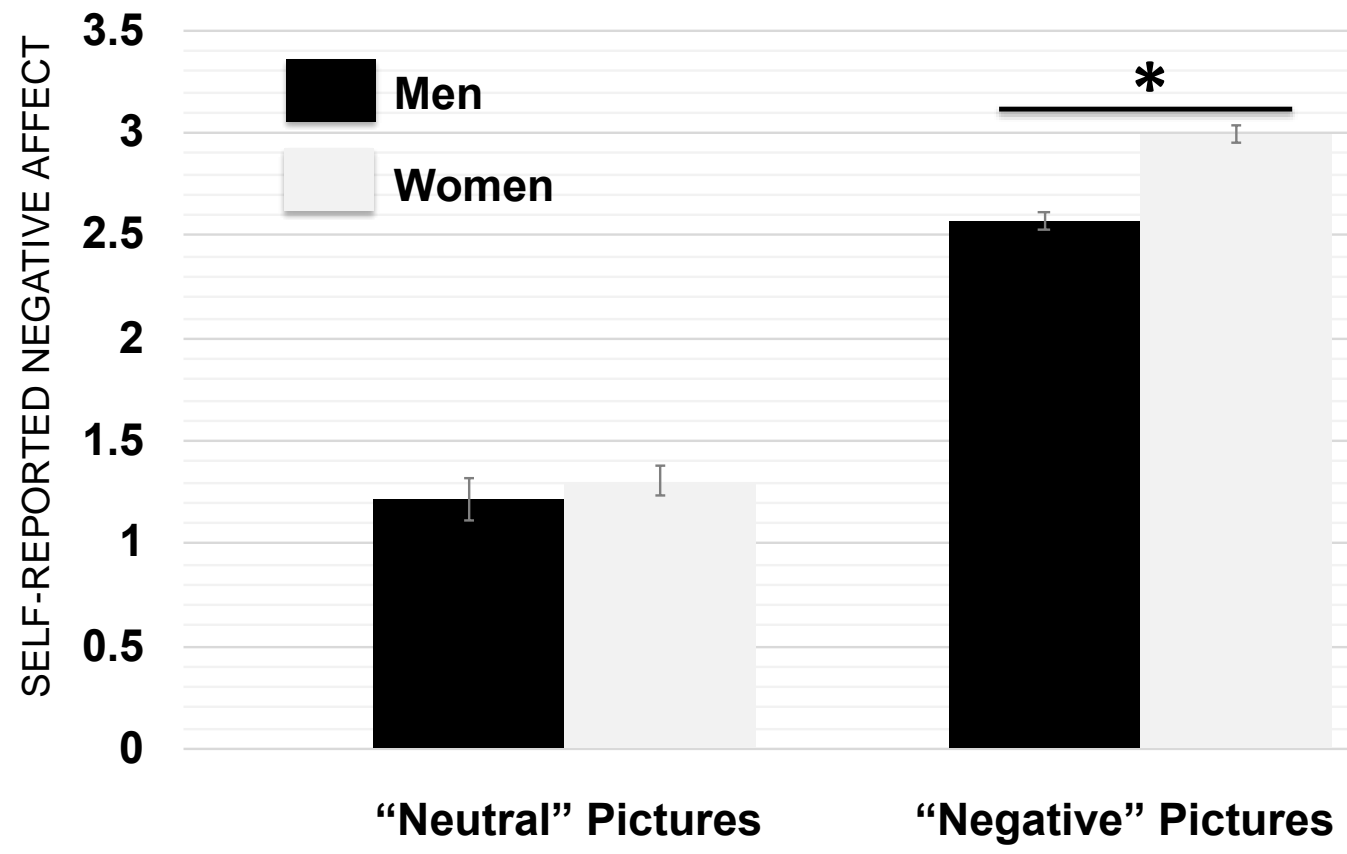

Figure 30: Gender differences in ratings of self-reported negative affect during the ER Baseline Task (part A). Each subject was asked to look and respond naturally to neutral (Neutral) and negative pictures (Negative). Error bars represent standard error the mean (SEM),

* represents $p<0.01$.

To examine subjective emotion ratings during emotion regulation (ER Task; part

B), as well as any gender differences in the ability to down-regulate negative emotion (Suppression Score), we conducted an independent samples t-test 
comparing the means of self-reported negative affect between genders (based on 5000 bootstrap samples) and tested for homogeneity of variances with Levene's test. The results showed a statistically significant difference between men's $(M=2.29, S D=.52)$ and women's $(M=2.64, S D=.47)$ negative ER ratings $t(45)=-0.35, p=0.02,95 \% \mathrm{Cl}[-.65$ to -.06$]$, but not between their neutral ER ratings $t(45)=-.75, p=0.45,95 \% \mathrm{Cl}[-0.45$ to 0.16$]$ (Figure 31 ) or their Suppression Score $t(45)=-.58, p=0.56,95 \% \mathrm{Cl}[-0.30$ to 0.17$]$ (Figure 32).

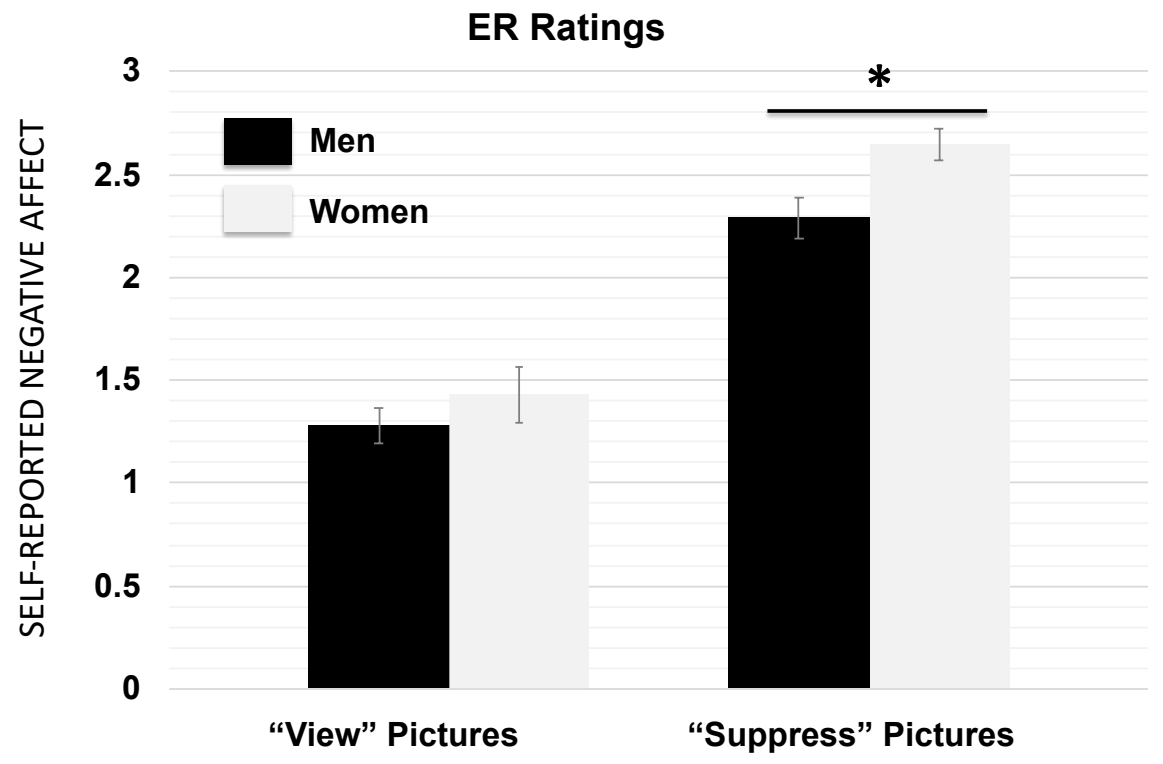

Figure 31: Gender differences in ratings of self-reported negative affect during the ER Task (part B). Each subject was asked to "simply view" the neutral pictures ("View" Pictures) and to "decrease or detach from the emotion" for negative pictures ("Suppress" Pictures). Error bars represent standard error of the mean (SEM), ${ }^{*}$ represents $p<0.05$. 


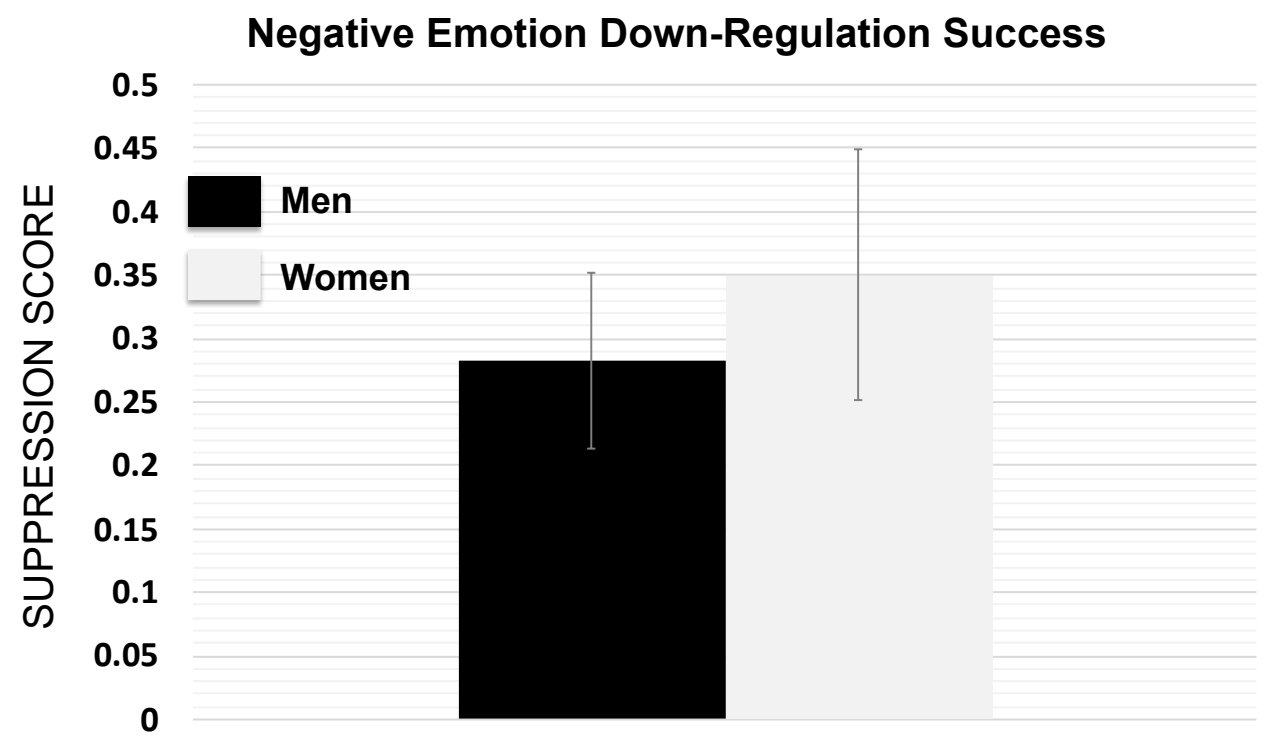

Figure 32: Gender differences in negative emotion down-regulation success (Suppression Score). The Suppression Score was derived from subtracting negative ER ratings (part $C$ ) from the earlier acquired negative baseline ratings (part A). Error bars represent standard error of the mean (SEM).

Finally, data regarding self-report ER strategy was analyzed. In order to determine inter-rater agreement, Cohen's kappa was run and returned an outstanding level of agreement for "Cognitive Reappraisal" (kappa = 0.885 (95\% $\mathrm{Cl} 0.76,0.80), \mathrm{p}<0.001)$ and "Attentional Control" (kappa $=0.866(95 \% \mathrm{Cl} 0.67$, $0.86), \mathrm{p}<0.001)$ and substantial for "Other" (kappa $=0.696(95 \% \mathrm{Cl} 0.59,0.73)$, $p<0.001)$. Then, to investigate any gender differences between the number of times a particular type of ER strategy was employed, we conducted a 2 (Gender) × 3 (type of ER Strategy: Cognitive Reappraisal, Attentional Control, Other) analysis of variance (based on 5000 bootstrap samples). Neither the main effect 
of gender nor the ER Strategy $\mathrm{X}$ gender interaction reached statistical

significance, therefore this variable was not used in subsequent analyses).

Neuroimaging Results

Gender Differences in Functional Connectivity during ER Baseline (part A)

To examine whether men and women exhibited neurobiological differences when viewing negative stimuli, we tested the ER Baseline (part A) prior to emotion regulation. As shown in Figure 33, Table 12) below, during the Negative Condition of the ER Baseline, compared to men, women showed increased FC between L FP, L Hip and L pPaHC, and between the R Hip and R AG.

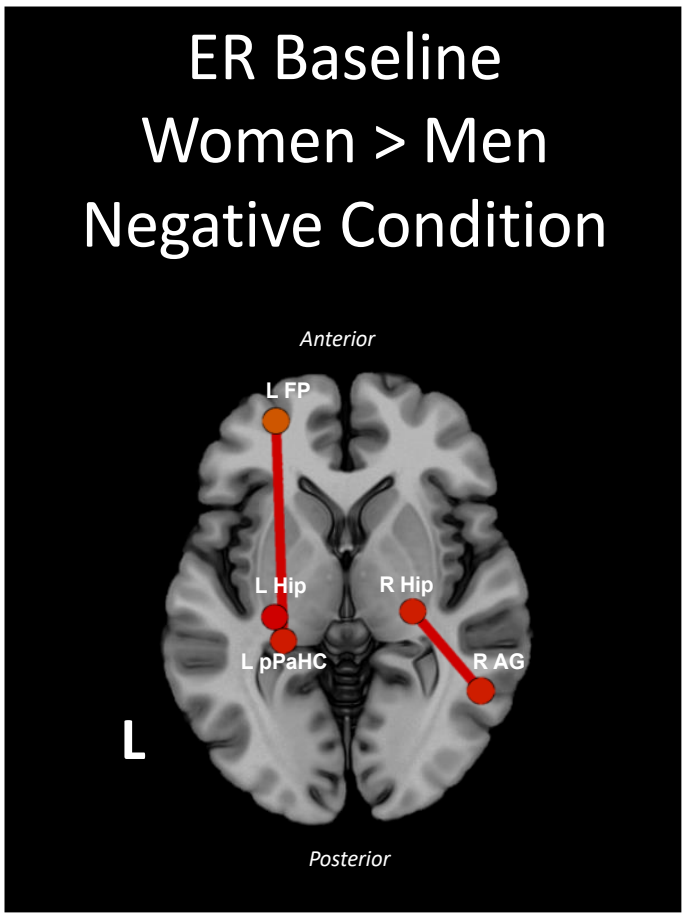

Figure 33: Gender differences in functional connectivity during the negative condition of ER Baseline.

Table 12 
ER Baseline

\begin{tabular}{lllll}
\hline ROI & ROI & T & p-FDR & Effec \\
L pPaHC & L Hip & -3.31 & 0.04 & 0.19 \\
L pPaHC & L FP & -3.24 & 0.04 & 0.14 \\
R Hip & R AG & -3.35 & 0.04 & 0.17
\end{tabular}

ROI Abbreviations: $p P a H C$ - posterior parahippocampal gyrus; Hip Hippocampus; FP - Frontal Pole; AG - Angular Gyrus.

Gender Differences in Functional Connectivity during ER Task (part B)

To examine whether men and women exhibited FC differences during emotion regulation (ER Task; part B), we examined gender differences with respect to suppression, as well as the correlation of FC during the ER Task with selfreported negative affect (ER Ratings, (part C)), and Suppression Score (ER Negative Baseline Ratings - Negative ER Ratings).

ER task

As shown in Figure 34, Table 13, women compared to men, during the Suppress Condition of the ER Task, showed increased FC between L IFG Oper and R pPaHC, between R IFG Oper and L pPaHC, and between L Hip and L pPaHC. In addition, women showed decreased connectivity between L pSMG, R pSMG and $R$ AG. 
Figure 34: Gender differences in functional connectivity during the suppress condition of the ER Task. Note: Color bar represents the strength of the t-statistic.

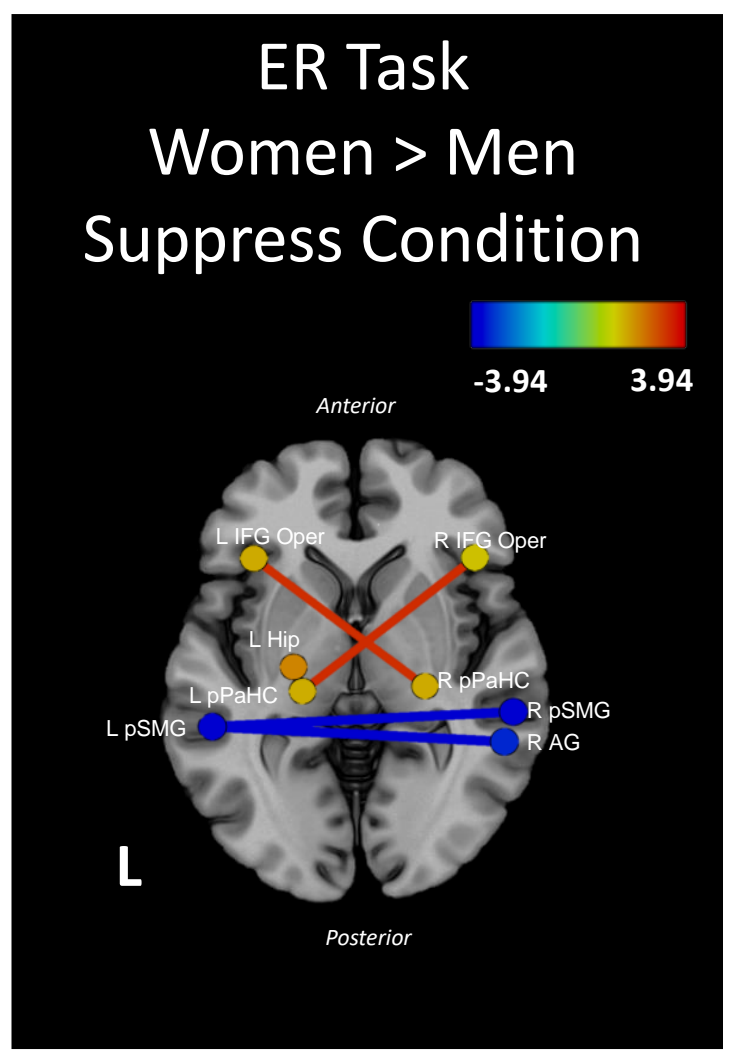

Table 13

\begin{tabular}{lllll}
\hline ER Task & & & & \\
\hline ROI & ROI & T & p-FDR & Effect Size \\
R AG & L pSMG & -3.94 & 0.007 & 0.27 \\
L pSMG & R pSMG & -3.86 & 0.009 & 0.31 \\
R pPaHC & L IFG Oper & 3.33 & 0.04 & 0.07 \\
L pPaHC & R IFG Oper & 3.38 & 0.02 & 0.12 \\
L pPaHC & L Hip & 3.26 & 0.02 & 0.18 \\
$\begin{array}{l}\text { ROI Abbreviations: AG - Angular Gyrus; } p S M G-\text { posterior supramarginal gyrus, } \\
\text { pPaHC- posterior parahippocampal gyrus; IFG Oper - Inferior frontal gyrus, } \\
\text { pars opercularis, Hip - Hippocampus }\end{array}$ & \multicolumn{4}{l}{}
\end{tabular}


ER Task Regressed with Self-Reported Negative Affect

When assessing the relationship between ER Task FC and self-reported negative affect (negative ratings from ER Rating Task (part C)), women's decreased self-reported negative affect compared to men's related to increased FC between R pSMG and L IFG Oper, and between R pSMG and R IFG Oper, L OFC and dACC during suppression (Figure 35, Table 14).

Figure 35: Gender differences in functional connectivity during the suppress condition of the ER Task, regressed with self-reported negative affect. Note: Color bar represents the strength of the t-statistic.

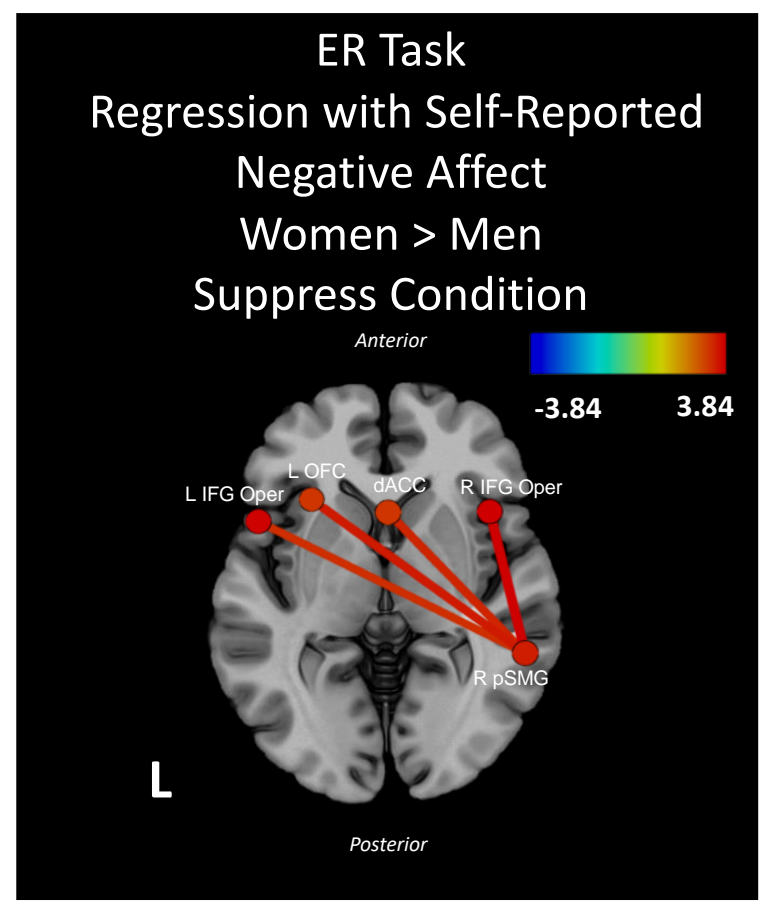

Table 14

ER Task Connectivity with Self-Reported Negative Affect

\begin{tabular}{lllll}
\hline ROI & ROI & T & p-FDR & Size \\
R pSMG & L IFG Oper & 3.24 & 0.01 & 0.41
\end{tabular}




$\begin{array}{lllll}\text { R pSMG } & \text { R IFG Oper } & 3.84 & 0.01 & 0.43 \\ \text { R pSMG } & \text { L OFC } & 3.49 & 0.01 & 0.35 \\ \text { R pSMG } & \text { dACC } & 3.36 & 0.01 & 0.35\end{array}$

ROI Abbreviations: $p S M G$ - posterior supramarginal gyrus; IFG Oper - inferior frontal gyrus, pars opercularis; OFC - orbitofrontal cortex; $d A C C$ - dorsal anterior cingulate gyrus.

\section{ER Task Regressed with Suppression Score}

Lastly, we assessed the relationship between ER Task FC and Suppression

Score (ER negative ratings (part C) subtracted from ER negative baseline

ratings). In the Suppress contrast, increased Suppression Score (better

suppression) in women, compared to men, was associated with increased FC

between L IFG tri and R IFG Oper, and between L IFG tri and R dACC (Figure

36, Table 15). 


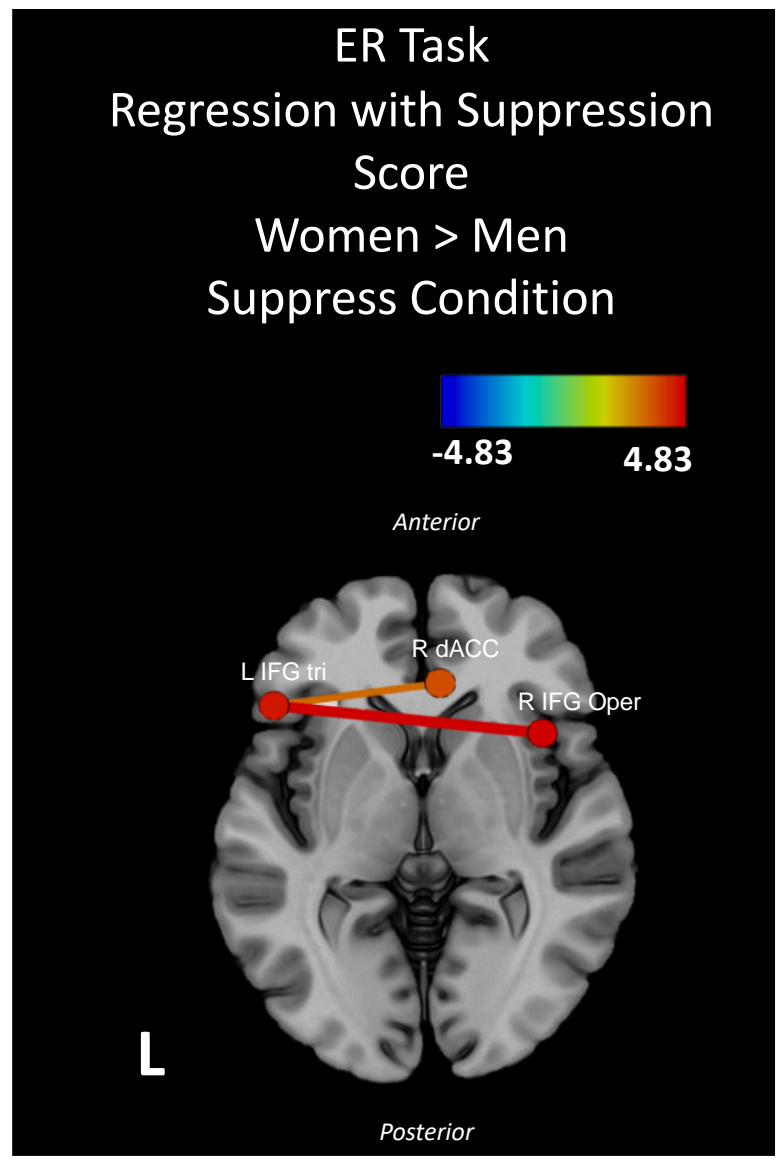

Figure 36: Gender differences in FC during the ER task, Suppress Condition, regressed with Suppression Score. Note: Color bar represents the strength of the t-statistic.

Table 15

ER Task Connectivity with Suppression Score

\begin{tabular}{lllll}
\hline ROI & ROI & T & p-FDR & Size \\
L IFG tri & R IFG Oper & 4.83 & 0.0005 & 0.63 \\
L IFG tri & R dACC & 3.18 & 0.0300 & 0.47
\end{tabular}

ROI Abbreviations: IFG tri - Inferior Frontal Gyrus, pars triangularis; IFG Oper Inferior Frontal Gyrus, pars opercularis; $d A C C$ - dorsal anterior cingulate gyrus. 


\section{Discussion}

The present fMRI study investigated whether gender differences existed in FC between brain regions underlying regulation of negative emotion during an ER task. Behaviorally, we observed that despite women rating the negative stimuli as more distressing compared to men, they had comparable Suppression Scores. However, the ER task revealed gender differences in FC. Specifically, in women enhanced ability to down-regulate negative emotion related to increased recruitment of a cingulo-opercular network, and in men, to posterior regions of a ventral attention network. Altogether, our results suggest men and women may utilize distinct executive control neural mechanisms in the control of negative emotion. To our knowledge, this is the first study to explore FC differences between men and women while engaging in ER and relate those differences to self-reported negative affect and suppression ability.

\section{Behavioral Findings}

Women self-reported as having more negative affect than men across tasks as we predicted, yet this difference disappeared in the process of downregulating negative emotion. Our results are consistent with previous findings showing women are more expressive, experientially reactive and sensitive to negative stimuli (Gard \& Kring, 2007; Hampson et al., 2006; Kring \& Gordon, 1998; H. Li et al., 2008). Moreover, our results mirror the Domes et al., 2010 and McRae et al., 2008 observations. Namely, that despite their heightened emotional reactivity, women suppressed their negative emotion to a comparable 
degree as men. This disassociation implies that there could be gender-related differences in the pattern of FC associated with ER. Furthermore, no gender differences were observed in what ER strategy was employed, in contrast to other research examining this topic (Tamres et al., 2002). We speculate that within our sample, a combination of ER strategies was used, although a paucity of research remains on such "coactive" emotion regulation, with most research to date simply reporting that individuals indeed deploy a variety of ER strategies (James J. Gross, 2002).

Neuroimaging Findings

We observed that while viewing negative stimuli (ER Baseline task), women exhibited increased FC between the left ventral FP, hippocampus and $\mathrm{pPaHC}$, as well as between right $\mathrm{AG}$ and hippocampus compared to men. Connectivity between ventral FP and hippocampus has been consistently associated with the recollection memory network (Adnan et al., 2016; Greenberg et al., 2005) and memory-based decision making (Weilbächer \& Gluth, 2017). In regard to the $\mathrm{pPaHC}$, this structure has been shown to be a key node of the recollection memory network, which together with lateral parietal cortices are preferentially engaged when individuals recall rich contextual details (Adnan et al., 2016), particularly about novel scenes (Howard et al., 2013). Furthermore, the AG is part of a largely right-lateralized, well validated ventral attention system specialized for the detection of behaviorally relevant stimuli (Corbetta \& Shulman, 2002). Notably, research suggests women are more likely to direct attention 
towards a novel item than men (Brown, 2013; Stoet, 2010). Together, our FC findings suggest that compared to men, women may engage a bottom-up emotional memory processing network when simply viewing negative stimuli, likely due to the allocation of attention to the highly salient stimuli.

During the regulation of negative emotion (ER Task), we observed women exhibited increased FC between the bilateral IFG and hippocampus and PPaHC, while men exhibited increased FC between the AG and bilateral pSMG. The IFG has been well established as a crucial brain region recruited during the volitional inhibition of affective response (Aron et al., 2014; Konishi et al., 1999; Ochsner et al., 2012), as well as pivotal in coordinating encoding processes in conjunction with the hippocampus (Addis \& McAndrews, 2006). The AG and pSMG, regions which underlie attention re-allocation to behaviorally salient stimuli (Corbetta \& Shulman, 2002), have previously been associated with a more automatic ER process (Viviani, 2013). Furthermore, a recent meta-analysis suggested the ER process may be explained not only as frontal regions inhibiting limbic regions, but also those underlying attention (Frank et al., 2014). In contrast to previous findings arguing men may utilize brain regions underlying top-down cognitive control to a greater extent than women during ER (Mak et al., 2009), our results suggest that women employ a bilateral top-down cingulo-opercular control network in order to suppress negative emotion. Regarding the FC findings in men, ER theorists have suggested the existence of an automatic (Mauss et al., 2007; M. L. Phillips et al., 2008), or unconscious variety of emotion regulation 
(Bargh \& Williams, 2007) which contrasts with the deliberate voluntary form typically interrogated (Ochsner \& Gross, 2005; Ochsner et al., 2012) and relies on the attentional reorienting mechanisms located in the ventral attention network (Viviani, 2013). Therefore, we speculate women may utilize a more deliberate ER process reliant on frontal regions, while men may employ a more efficient (less cognitively effortful) ER process dependent on regions within the posterior regions of the ventral attention network.

In parallel, we found decreased self-reported negative affect in women (compared to men) related to increased connectivity between right pSMG and left IFG, dACC and OFC. The dACC lies in a unique anatomical position, with connections to both the limbic system and PFC, and has an important role in integrating neuronal circuitry for affect regulation (Stevens et al., 2011). Moreover, the dACC plays a crucial role in conflict monitoring (Lau et al., 2006; Stuss \& Levine, 2002; Thomsen et al., 2005) as well as integration of attentional and emotional stimuli (Yamasaki et al., 2002). Hence, dACC connectivity with frontal and parietal structures in our results may point to a deliberate effort on the part of women to resolve the heightened emotional conflict during the ER process. Similarly, previous studies implicate the OFC in top-down modulation of autonomic responses to emotional experiences (Ohira et al., 2006; Mary L. Phillips et al., 2003), and it appears larger in women than men (Gur et al., 2002). Therefore, our findings suggest decreased self-reported negative affect in women may relate to the recruitment of a fronto-parietal top-down control 
network in order to modulate the autonomic physiological responses evoked by the highly salient emotional stimuli.

Lastly, we observed that in women (compared to men), better suppression of negative emotion related to increased connectivity between bilateral IFG and R dACC. Along with the IFG, robust evidence exists for the recruitment of the dACC during the ER process as a conflict monitoring tool (Mak et al., 2009; McRae et al., 2008; Ochsner \& Gross, 2005; Ochsner et al., 2012; Weissman et al., 2005). That is, the dACC may underlie monitoring cognitive conflict and recruits other prefrontal regions to resolve this conflict when necessary (Botvinick et al., 2004; J. Fan et al., 2003), putatively in order to reduce amygdala activity (Etkin et al., 2006). Previous studies investigating gender differences in ER suggest women tend to utilize emotion-associated brain areas, while men tend to utilize cognitive ones (Kohn et al., 2014; Mak et al., 2009), and even that men may possess a greater ability to regulate their emotions compared to women (Kong et al., 2014). In contrast, our novel FC findings demonstrate that women downregulate negative emotion as successfully as men by recruiting a cinguloopercular network.

To our knowledge, these results provide the first FC evidence that indicate women suppress negative emotion as well as men by exhibiting a cinguloopercular network, while men exhibit a posterior region of the ventral attentional network. Since women reported increased negative affect compared to men, we suggest that the heightened emotional response required utilization of the top- 
down cingulo-opercular control network to effectively downregulate the negative emotion. Men recruited posterior regions of the ventral attentional network while suppressing negative emotion, perhaps due to finding the negative stimuli less aversive as women, and conceivably requiring an efficient and automatic form of ER. The FC findings contribute an alternate avenue for investigating gender differences within the neural correlates of ER, which may explain women's greater prevalence of developing affective disorders, particularly depression and anxiety (Kessler et al., 1993; Leach et al., 2008; Nolen-Hoeksema, 2001, 2012; Thomsen et al., 2005). Indeed, research suggests that in major depressive disorder, functional and structural abnormalities within cingulo-opercular pathways could contribute to maladaptive forms of self-focused processing and rumination (Pizzagalli, 2011).

\section{Limitations and Future Directions}

A few limitations in our study should be noted. The lack of control of hormonal cycle or contraception method for female participants poses a problem. Indeed, it has been shown that social processes, and in particular the neural response to emotion regulation, may vary as a function of hormonal phase of the women (Dan et al., 2019). In the same vein, testosterone has been shown to modulate brain networks important for social-emotional processing in men (Votinov et al., 2020) and should be taken account in future investigations. It should also be noted that we had small, unequal sample sizes which may reduce statistical power (Rusticus \& Lovato, 2014). Further, due to the fact our functional 
connectivity analysis included a large number of individual ROls, caution should be exercised in order to avoid the reverse inference fallacy (Poldrack, 2000). However, we assert that sensitivity and specificity are high enough, and therefore our analysis has a high positive predictive value - that is, a high likelihood of implying the down regulation of negative emotion (Poldrack, 2011). Additionally, subjective negative affect ratings may not be the most valid measures to reflect emotional experiences, and that the inclusion of physiological responses to emotion could have provided a more reliable index (Ohira et al., 2006).

\section{Summary}

In conclusion, the present study demonstrated gender-specific FC patterns associated with negative emotion suppression during an ER task, which corresponded with self-reported negative affect and its downregulation success in a group of healthy individuals. Specifically, the results indicated women utilize a cingulo-opercular network to downregulate negative emotion, while men employ posterior regions of the ventral attention network, with comparable suppression outcomes. We speculate the dissociation in the use of executive control mechanisms is likely due to increased emotional reactivity commonly observed among women and an enhanced need to resolve heightened emotional conflict. The findings may provide a better understanding of the mechanisms underlying gender differences with respect to ER. Enhanced knowledge of gender-specific neural bases of ER may help illuminate women's vulnerability to 
affective disorders and critically, may facilitate the design of personalized therapeutic interventions, for instance utilizing neurofeedback (Linhartová et al., 2019), to target gender-specific neural networks underlying ER. 


\section{CHAPTER VI: GENERAL DISCUSSION}
"Sustained shortfalls in emotional intelligence are, sadly, no minor matter. There are few catastrophes, in our own lives or in those of nations, that do not ultimately have their origins in emotional ignorance."

Alain de Botton

An Integrative Neurocognitive El Model

Through the different studies this dissertation presented, we utilized a myriad of neuroimaging analyses to elucidate the neural correlates of the proposed cognitive-emotional El framework, comprising empathy and ER. In Experiment 1, we demonstrated that these processes are intimately related through the temporal interplay of the CEN, DMN and SN, and that these flexible network (re)configurations relate to trait empathy and ER. In Experiment 2, we showed that empathy is related to sensitivity to one's own bodily changes (interoception), which is anchored in regions of the SN. In Experiment 3, we suggested that enhanced bottom-up processing seen in empathy and worry, reliant on the SN, contributed to trait anxiety due to decreased activation of CEN regions. In Experiment 4, we demonstrated that in a healthy adolescent sample, decreased structural brain measurements and increased estradiol related to decreased ER. In Experiment 5, we showed men and women use different parts 
of the CEN to down-regulate negative emotion, albeit with comparable suppression scores. Collectively, the studies imply that harmonious socioemotional communication may arise from effective communication between neurocircuitry underlying the CEN, DMN and SN, and is furthermore modulated by gender, as well as physiological and psychological characteristics. In addition, we propose a role for the $\mathrm{SN}$ as a "switch" between internal and external attention which may contribute to favorable socioemotional communication outcomes. Therefore, this dissertation contributes a neural basis for the proposed cognitive-emotional El framework, bridging the conceptual divide between "emotional" and "cognitive" El processes and creating a new integrative

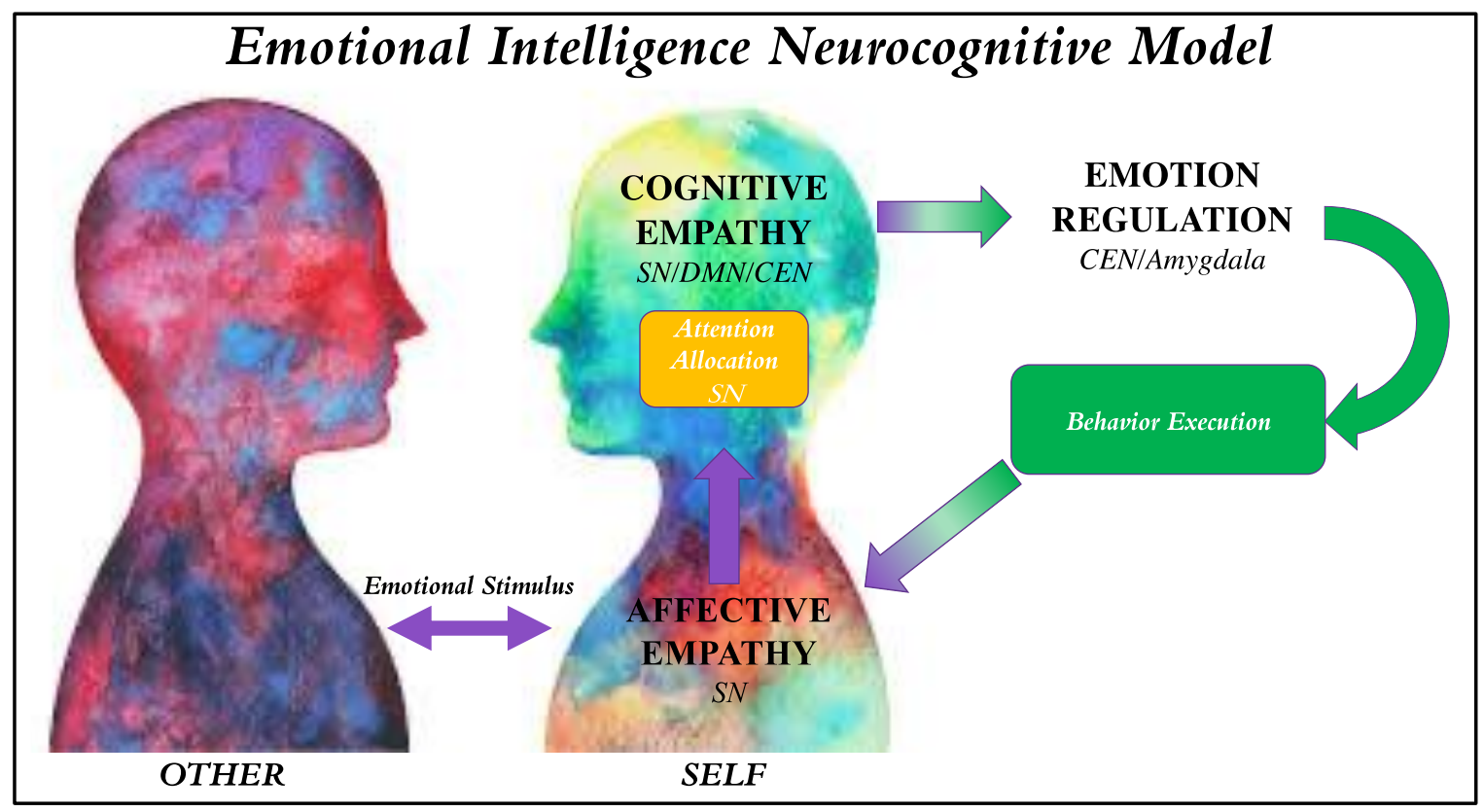

neurocognitive El model (Figure 37).

Figure 37: Schematic illustration of the proposed neurocognitive El model. As the self observes emotions in the other, regions anchored in the $\mathrm{SN}$ contribute to automatically sharing the emotion (Affective Empathy). Facilitated by the SN, attention is externally modulated, whereby understanding emotion (Cognitive Empathy) is achieved by simulating the observed emotion reliant on the interplay 
between the SN, DMN and CEN. To modulate the resultant emotion, CEN brain regions come online to down-regulate limbic regions such as the amygdala, which eventually lead to behavior execution. Based on iterative observations of the other, the cycle may begin again to produce desired emotional attunement.

While real-world emotional attunement reflects a bi-directional process, in which the state of the self and other may both change in response to one another, for the sake of parsimony, we focus here only on the emotional landscape of the self. It is also important to note that an individual's understanding of, and response to, another's emotions reflects both their capacity as well as their propensity to engage in these attunement processes (Cameron et al., 2017; Zaki, 2014).

During an emotional interchange, the self observes concrete cues from the other such as facial, bodily or vocal expressions that prompts creation of an embodied emotion in the self (Affective Empathy). Although previous findings anchor this type of automatic processing solely in the SN (Decety et al., 2013), our results extend this conclusion by indicating that Affective Empathy may arise from the dynamic interplay between the SN, CEN and DMN (Experiment 1); and furthermore, that Affective Empathy shares rsFC with interoception, the awareness of changes in bodily sensations (Experiment 2). Affective Empathy, therefore, enables a direct mapping of another's emotion on the brain system of the observer, without an explicit need for complex cognition (Adolphs, 2002; Carr et al., 2003; De Waal \& Preston, 2017; Jabbi \& Keysers, 2008). To wit, this type of rudimentary emotional processing serves as the blueprint from which the self can draw experience to better understand the other's emotional state. 
In addition to the $\mathrm{SN}$ being implicated in monitoring and processing body state changes, it also plays a critical and causal role in engaging the brain's attentional, working memory and higher-order control processes (CEN) while disengaging other systems that are not immediately task relevant (Menon \& Uddin, 2010). In the context of the neurocognitive El model proposed, the self's $\mathrm{SN}$ may generate a state of heightened physiological awareness due to the other's emotional state, which subsequently results in either internal or external attention allocation. In the case of shifting attention internally, the CEN and DMN may come online for better understanding and subsequently regulating the instantiated emotion. In the case of shifting attention externally, sustained use of the SN may be needed to gather additional emotional information from the other. Given its crucial role in attention allocation, atypical SN activity may lead to psychopathology. Indeed, SN hyperactivity has been implicated in anxiety disorders, suggesting that when the SN goes into overdrive, pathology subsequently results (Feinstein et al., 2006; Paulus \& Stein, 2006; M. B. Stein et al., 2007). In line with this thinking, we demonstrated that increased anxiety in Experiment 3 was related to increased SN, but decreased CEN processing. Therefore, in the context of the neurocognitive El model, we propose the SN has a crucial role in triggering a cascade of cognitive control signals that have a major impact on how emotional stimuli are subsequently processed.

Once concrete emotional cues are available and attention is directed inward, various facets of cognitive control enable the observer to simultaneously 
represent their own and the other's emotional state and inhibit their default egocentric perspective in order to take the perspective of the other - i.e., Cognitive Empathy (Chakrabarti and Baron-Cohen, 2006; Coplan and Goldie, 2012; O'Connell et al., 2013). Previous research suggests that Cognitive Empathy involves switching between the representations of self and other in service of inferences based on emotional cues that require temporal binding of the available information; therefore, it relies on brain regions anchored in the SN, CEN and DMN (Atique et al., 2011; Corradi-Dell'Acqua et al., 2014; Goel et al., 1995; Lamm et al., 2011; Morelli et al., 2014; Van Overwalle, 2009). While in agreement, our studies provide novel perspectives on this claim using enhanced neuroimaging methodologies. Namely, the dynFC findings from Experiment 1 suggest mentalizing is indeed an effortful process that may not be able to occur at the same time as other taxing cognitive processes. In addition, the rsBOLD findings from Experiment 2 suggest flexibility between networks underlying mentalizing relates to an improved sense of interconnectedness between one's own mind and body. Therefore, taking the other's perspective needs more cortical resources and time compared to the automatic sharing of emotion, i.e., Affective Empathy.

Additionally, research suggests Cognitive Empathy shares neural resources with ER (Kalisch, 2009; Ozonoff et al., 1991; Sabbagh et al., 2006; Urry et al., 2009), which is thought to be mediated by neural circuits involving regions anchored in the CEN that putatively down-regulate the amygdala 
(Davidson et al., 2000; Etkin et al., 2015). Our studies corroborate this evidence, and additionally contribute valuable information regarding gender differences within this process, and ER's relationship to empathy and estradiol. In Experiment 1, we show a behavioral and dynFC overlap between Cognitive Empathy and Cognitive Reappraisal. In Experiment 4, we show that in an adolescent sample, increased estradiol and decreased structural brain measurements were related to decreased executive functions (i.e., ER). Finally, in Experiment 5, we show men and women utilize different parts of the CEN to down-regulate negative emotion. Therefore, we provide evidence that suggests ER is a complex cognitive process that may be modulated by gender, physiological and psychological variables.

Following ER due to another's emotional distress, behavior execution may occur (for example, comforting the other); or the iterative process may continue based on sensory information gathered from the other, processed by the SN. We also note that previous research shows reward value assigned to the observed other modulates the extent of embodiment and related neural responses in an observer (Sims et al., 2014; Trilla et al., 2015), therefore, this model is highly context dependent.

\section{Clinical Implications}

The proposed integrative neurocognitive El model has clear applications for clinical disorders arising from deficits in either empathy, ER, or both. For 
example, mentalization-based approaches to treating borderline personality disorder (Fonagy \& Luyten, 2009) depend on representing and attending to the emotions of self and others. Cognitive-behavioral therapy (Beck, 2011) can also be understood to promote the use of higher order executive control processes. Moreover, promising research suggests these El constructs can be trained, leading to improved psychological outcomes (Bagheri et al., 2017). The (demonstrably trainable) emotion abilities we have discussed have been found to be lower in several clinical populations, including major depression (Demiralp et al., 2012), social anxiety disorder (Kashdan \& Farmer, 2014), autism spectrum disorder (Erbas et al., 2013), eating disorders (Selby et al., 2013), and borderline personality disorder (Suvak et al., 2011). In combination with the recently suggested genetic links of EI (Vladimir et al., 2019), this integrative neurocognitive El model illuminates avenues for treatment such as deep brain stimulation (DBS) to target a subset of networks underlying specific deficits, rather than single brain regions (Bewernick et al., 2010; Ramasubbu et al., 2018).

\section{Future Directions}

The proposed neurocognitive El model can also be useful in the design of future El training programs. For example, evidence already exists that mindfulness meditation improves El by increasing cognitive and emotional functioning (Charoensukmongkol, 2014; Chu, 2010). Therefore, implementation 
of meditation techniques, in combination with real-time fMRI approaches, and psycho-physiological measurements (for ex., skin conductance) could improve socioemotional communication. It is worth noting given the problems of "reverse inference" associated with neuroimaging (Poldrack, 2011) there are important limitations when attempting to infer psychological differences based on observed neural differences; thus, this particular use of our model should be treated with some caution. With regard to emotion recognition, the model suggests that a primary training target may be the sculpting of new attentional dispositions. That is, if individuals were trained to shift attention to the most emotionally informative facial, bodily, and voice cues in particular contexts, emotion recognition, and subsequently empathy, may improve. With regard to improving ER, our model suggests that ER skills are part of the higher-order cognitive repertoire, and thus ER may benefit from greater emotional working memory capacity; it is unclear at present, however, whether training programs can effectively increase working memory capacity for the emotions of self and others, thus research on this topic is needed.

\section{Conclusion}

El is a crucial ability that allows the processing of emotion in both self and others, leading to harmonious social encounters. The work presented here contributes important neurobiological insights in regard to two constructs underlying El: empathy and ER. Firstly, we demonstrate that networks underlying 
these constructs dynamically communicate to support optimal emotional processing, reconciling the seemingly opposing perspectives of functional segregation and integration. Secondly, we show empathy has neurobiological links to interoception and anxiety, providing evidence for theories positing relationships among these constructs. Thirdly, we illustrate that ER is a complex higher-order cognitive process that may be modulated by brain structure and function, as well as hormones and gender. Finally, the proposed integrative neurocognitive El model based on this data can serve as a blueprint for developing El training programs and offers a (currently lacking) performancebased neurobiological cognitive test of El.

Perhaps now more than ever, there is a dire need for embracing the nuances of human emotion. In addition to the social isolation experienced by many individuals during the Covid-19 global pandemic, social unrest and political divisiveness arose during this time, fueled by the tendency to see groups as not belonging, fitting in or just being different, otherwise known as "othering" (Brons, 2015). This disturbing pattern also affects the environment, as experts estimate that humans are driving one million species of plants and animals to extinction by refusing to cooperate to pass conservation legislature (Tollefson, 2019).

Understanding how other's emotional landscapes are different from ours not only offers enormous interpersonal benefits, but has the potential to ripple throughout society at large, resulting in a more harmonious and peaceful world. 


\section{REFERENCES}

Adamaszek, M., D’Agata, F., Ferrucci, R., Habas, C., Keulen, S., Kirkby, K. C., Leggio, M., Mariën, P., Molinari, M., \& Moulton, E. (2017). Consensus paper: cerebellum and emotion. The Cerebellum, 16(2), 552-576.

Addis, D. R., \& McAndrews, M. P. (2006). Prefrontal and hippocampal contributions to the generation and binding of semantic associations during successful encoding. Neurolmage, 33(4), 1194-1206. https://doi.org/10.1016/j.neuroimage.2006.07.039

Adnan, A., Barnett, A., Moayedi, M., McCormick, C., Cohn, M., \& McAndrews, M. P. (2016). Distinct hippocampal functional networks revealed by tractography-based parcellation. Brain Structure and Function, 221(6), 2999-3012. https://doi.org/10.1007/s00429-015-1084-x

Adolfi, F., Couto, B., Richter, F., Decety, J., Lopez, J., Sigman, M., Manes, F., \& Ibáñez, A. (2017). Convergence of interoception, emotion, and social cognition: A twofold fMRI meta-analysis and lesion approach. Cortex, 88, 124-142. https://doi.org/10.1016/j.cortex.2016.12.019

Adolphs, R. (2002). Neural systems for recognizing emotion. Current Opinion in Neurobiology, 12(2), 169-177. https://doi.org/10.1016/S09594388(02)00301-X

Adolphs, R., Damasio, H., Tranel, D., Cooper, G., \& Damasio, A. R. (2000). A role for somatosensory cortices in the visual recognition of emotion as revealed by three-dimensional lesion mapping. Journal of Neuroscience, 20(7), 2683-2690. https://doi.org/10.1523/jneurosci.20-07-02683.2000

Aliakbari, M., Juibari, A. K., Amirabadi, F., Shaghaghi, F., Zare, N., \& Khaleghi, F. (2013). The role of Theory of Mind and executive function in predicting empathy. In Advances in Cognitive Science (Vol. 15, Issues 2[58]; 58, pp. 110). Institute for Cognitive Science Studies.

http://ovidsp.ovid.com/ovidweb.cgi?T=JS\&CSC=Y\&NEWS=N\&PAGE=fulltex $\mathrm{t} \& \mathrm{D}=\mathrm{psyc} 10 \& \mathrm{AN}=2013-44426-$

001\%5Cnhttp://oxfordsfx.hosted.exlibrisgroup.com/oxford?sid=OVID:psycdb \&id=pmid:\&id=doi:\&issn=1561-

$4174 \&$ isbn $=\&$ volume $=15 \&$ issue $=2[58] \&$ spage $=1$ \&page $=1$ -

10\&date $=2013 \&$ titl

Allen, E. A., Damaraju, E., Plis, S. M., Erhardt, E. B., Eichele, T., \& Calhoun, V. D. (2014). Tracking whole-brain connectivity dynamics in the resting state. Cerebral Cortex, 24(3), 663-676. https://doi.org/10.1093/cercor/bhs352 
Almey, A., Milner, T. A., \& Brake, W. G. (2015). Estrogen receptors in the central nervous system and their implication for dopamine-dependent cognition in females. Hormones and Behavior, 74, 125-138.

https://doi.org/10.1016/j.yhbeh.2015.06.010

Amodio, D. M., \& Frith, C. D. (2006). Meeting of minds: the medial frontal cortex and social cognition. Nature Reviews Neuroscience, 7(4), 268-277. https://doi.org/10.1038/nrn1884

Anderson, M. (2014). After phrenology: neural reuse and the interactive brain (Issue 8). MIT Press. https://doi.org/10.1080/00140139.2017.1314655

Andersson, J., Smith, S., \& Jenkinson, M. (2008). FNIRT-FMRIB's non-linear image registration tool. 14Th Annual Meeting of the Organization for Human Brain Mapping (OHBM), 15-19.

Andrews-Hanna, J. R. (2011). The Brain's Default Network and Its Adaptive Role in Internal Mentation. The Neuroscientist, 18(3), 251-270. https://doi.org/10.1177/1073858411403316

Antshel, K. M., Conchelos, J., Lanzetta, G., Fremont, W., \& Kates, W. R. (2005). Behavior and corpus callosum morphology relationships in velocardiofacial syndrome (22q11.2 deletion syndrome). Psychiatry Research: Neuroimaging, 138(3), 235-245. https://doi.org/10.1016/j.pscychresns.2005.02.003

Arain, M., Haque, M., Johal, L., Mathur, P., Nel, W., Rais, A., Sandhu, R., \& Sharma, S. (2013). Maturation of the adolescent brain. Neuropsychiatric Disease and Treatment, 9, 449-461. https://doi.org/10.2147/NDT.S39776

Armbruster-Genç, D. J. N., Ueltzhöffer, K., \& Fiebach, C. J. (2016). Brain signal variability differentially affects cognitive flexibility and cognitive stability. Journal of Neuroscience, 36(14), 3978-3987. https://doi.org/10.1523/JNEUROSCI.2517-14.2016

Aron, A. R., Robbins, T. W., \& Poldrack, R. A. (2014). Inhibition and the right inferior frontal cortex: One decade on. Trends in Cognitive Sciences, 18(4), 177-185. https://doi.org/10.1016/j.tics.2013.12.003

Asato, M. R., Terwilliger, R., Woo, J., \& Luna, B. (2010). White matter development in adolescence: a DTI study. Cerebral Cortex (New York, N.Y. : 1991), 20(9), 2122-2131. https://doi.org/10.1093/cercor/bhp282

ASHTARI, M. (2011). Anatomy and functional role of the inferior longitudinal fasciculus: a search that has just begun. Developmental Medicine \& Child Neurology, 54(1), 6-7. https://doi.org/10.1111/j.1469-8749.2011.04122.x

Asplund, C. L., Todd, J. J., Snyder, A. P., \& Marois, R. (2010). A central role for the lateral prefrontal cortex in goal-directed and stimulus-driven attention. Nature Neuroscience, 13(4), 507-512. https://doi.org/10.1038/nn.2509

Austin, E. J., Saklofske, D. H., Huang, S. H. S., \& McKenney, D. (2004). Measurement of trait emotional intelligence: Testing and cross-validating a modified version of Schutte et al.'s (1998) measure. Personality and Individual Differences, 36(3), 555-562. https://doi.org/10.1016/S01918869(03)00114-4 
Bagheri, Z., Mohd Kosnin, A., \& Besharat, M. A. (2017). Improving Emotion Regulation skills through an Emotional Intelligence Training Course. Khazar Journal of Humanities and Social Sciences, 19(4), 36-48. https://doi.org/10.5782/2223-2621.2016.19.4.36

Banks, S. J., Eddy, K. T., Angstadt, M., Nathan, P. J., \& Luan Phan, K. (2007). Amygdala-frontal connectivity during emotion regulation. Social Cognitive and Affective Neuroscience, 2(4), 303-312. https://doi.org/10.1093/scan/nsm029

Bar-On, R., Tranel, D., Denburg, N. L., \& Bechara, A. (2003). Exploring the neurological substrate of emotional and social intelligence. Brain, 126(8), 1790-1800. https://doi.org/10.1093/brain/awg177

Barbey, A. K., Koenigs, M., \& Grafman, J. (2011). Orbitofrontal contributions to human working memory. Cerebral Cortex, 21(4), 789-795. https://doi.org/10.1093/cercor/bhq153

Barbey, A. K., Koenigs, M., \& Grafman, J. (2013). Dorsolateral prefrontal contributions to human working memory. Cortex, 49(5), 1195-1205. https://doi.org/10.1016/j.cortex.2012.05.022

Bardi, L., Desmet, C., Nijhof, A., Wiersema, J. R., \& Brass, M. (2017). Brain activation for spontaneous and explicit false belief tasks overlaps: New fMRI evidence on belief processing and violation of expectation. Social Cognitive and Affective Neuroscience, 12(3), 391-400. https://doi.org/10.1093/scan/nsw143

Bargh, J. A., \& Williams, L. E. (2007). The case for nonconscious emotion regulation. In J.J. Gross (Ed.), Handbook of emotion regulation (pp. 429445). Guilford Press.

Barnes, L. L. B., Harp, D., \& Jung, W. S. (2002). Reliability Generalization of Scores on the Spielberger State-Trait Anxiety Inventory. Educational and Psychological Measurement, 62(4), 603-618. https://doi.org/10.1177/0013164402062004005

Baron-Cohen, S., Jolliffe, T., Mortimore, C., \& Robertson, M. (1997). Another advanced test of theory of mind: Evidence from very high functioning adults with autism or Asperger syndrome. In Journal of Child Psychology and Psychiatry and Allied Disciplines (Vol. 38, Issue 7, pp. 813-822). https://doi.org/10.1111/j.1469-7610.1997.tb01599.x

Baron-Cohen, S., Knickmeyer, R. C., \& Belmonte, M. K. (2005). Sex Differences in the Brain: Implications for Explaining Autism. Science, 310(5749), 819823. https://doi.org/10.1126/science. 1115455

Baron-Cohen, S., Ring, H., Chitnis, X., Wheelwright, S., Gregory, L., Williams, S., Brammer, M., \& Bullmore, E. (2006). fMRI of parents of children with Asperger Syndrome: A pilot study. Brain and Cognition, 61(1), 122-130. https://doi.org/10.1016/j.bandc.2005.12.011

Baron-Cohen, S., \& Wheelwright, S. (2004). The empathy quotient: An investigation of adults with asperger syndrome or high functioning autism, and normal sex differences. In Journal of Autism and Developmental 
Disorders (Vol. 34, Issue 2, pp. 163-175).

https://doi.org/10.1023/B:JADD.0000022607.19833.00

Baron-Cohen, S., Wheelwright, S., Hill, J., Raste, Y., \& Plumb, I. (2001). The

"Reading the Mind in the Eyes" Test revised version: a study with normal adults, and adults with Asperger syndrome or high-functioning autism. The Journal of Child Psychology and Psychiatry and Allied Disciplines, 42(2), 241-251.

Barrett, L. F. (2004). Feelings or words? Understanding the content in self-report ratings of experienced emotion. Journal of Personality and Social Psychology, 87(2), 266-281. https://doi.org/10.1037/0022-3514.87.2.266

Barrett, L. F., Bliss-Moreau, E., Quigley, K. S., \& Aronson, K. R. (2004). Interoceptive sensitivity and self-reports of emotional experience. Journal of Personality and Social Psychology, 87(5), 684-697. https://doi.org/10.1037/0022-3514.87.5.684

Barrett, L. F., \& Satpute, A. B. (2013). Large-scale brain networks in affective and social neuroscience: Towards an integrative functional architecture of the brain. Current Opinion in Neurobiology, 23(3), 361-372. https://doi.org/10.1016/j.conb.2012.12.012

Barrett, L. F., \& Simmons, W. K. (2015). Interoceptive predictions in the brain. Nature Reviews Neuroscience, 16(7), 419-429. https://doi.org/10.1038/nrn3950

Baumann, O., \& Mattingley, J. B. (2012). Functional topography of primary emotion processing in the human cerebellum. Neurolmage, 61(4), 805-811. https://doi.org/10.1016/j.neuroimage.2012.03.044

Baur, V., Hänggi, J., Langer, N., \& Jäncke, L. (2013). Resting-State Functional and Structural Connectivity Within an Insula-Amygdala Route Specifically Index State and Trait Anxiety. Biological Psychiatry, 73(1), 85-92. https://doi.org/10.1016/j.biopsych.2012.06.003

Bechara, A., Damasio, A. R., Damasio, H., \& Anderson, S. W. (1994). Insensitivity to future consequences following damage to human prefrontal cortex. Cognition, 50(1-3), 7-15. https://doi.org/10.1016/00100277(94)90018-3

Bechara, A., Damasio, H., \& Damasio, A. R. (2000). Emotion, decision making and the orbitofrontal cortex. Cerebral Cortex, 10(3), 295-307. https://doi.org/10.1093/cercor/10.3.295

Beck, J. S. (2011). Cognitive behavior therapy. New York: Basics and beyond. Guilford Publication.

Behrens, T. E. J., Woolrich, M. W., Jenkinson, M., Johansen-Berg, H., Nunes, R. G., Clare, S., Matthews, P. M., Brady, J. M., \& Smith, S. M. (2003). Characterization and propagation of uncertainty in diffusion-weighted MR imaging. Magnetic Resonance in Medicine, 50(5), 1077-1088. https://doi.org/10.1002/mrm.10609

Behzadi, Y., Restom, K., Liau, J., \& Liu, T. T. (2007). A component based noise correction method (CompCor) for BOLD and perfusion based fMRI. 
Neurolmage, 37(1), 90-101. https://doi.org/10.1016/j.neuroimage.2007.04.042

Benjamini, Y., \& Hochberg, Y. (1995). Controlling the False Discovery Rate: A Practical and Powerful Approach to Multiple Testing. Journal of the Royal Statistical Society: Series B (Methodological), 57(1), 289-300. https://doi.org/10.1111/j.2517-6161.1995.tb02031.x

Berking, M., Wirtz, C. M., Svaldi, J., \& Hofmann, S. G. (2014). Emotion regulation predicts symptoms of depression over five years. Behaviour Research and Therapy, 57(1), 13-20. https://doi.org/10.1016/j.brat.2014.03.003

Bernhardt, B. C., \& Singer, T. (2012). The neural basis of empathy. Annual Review of Neuroscience, 35(1), 1-23. https://doi.org/10.1146/annurevneuro-062111-150536

Bewernick, B. H., Hurlemann, R., Matusch, A., Kayser, S., Grubert, C., Hadrysiewicz, B., Axmacher, N., Lemke, M., Cooper-Mahkorn, D., \& Cohen, M. X. (2010). Nucleus accumbens deep brain stimulation decreases ratings of depression and anxiety in treatment-resistant depression. Biological Psychiatry, 67(2), 110-116.

Biesbroek, J. M., Weaver, N. A., Hilal, S., Kuijf, H. J., Ikram, M. K., Xu, X., Tan, B. Y., Venketasubramanian, N., Postma, A., Biessels, G. J., \& Chen, C. P. L. H. (2016). Impact of Strategically Located White Matter Hyperintensities on Cognition in Memory Clinic Patients with Small Vessel Disease. PloS One, 11(11), e0166261-e0166261. https://doi.org/10.1371/journal.pone.0166261

Bilevicius, E., Kolesar, T. A., Smith, S. D., Trapnell, P. D., \& Kornelsen, J. (2018). Trait emotional empathy and resting state functional connectivity in default mode, salience, and central executive networks. Brain Sciences, 8(7). https://doi.org/10.3390/brainsci8070128

Bimonte, H. A., \& Denenberg, V. H. (1999). Estradiol facilitates performance as working memory load increases. Psychoneuroendocrinology, 24(2), 161173. https://doi.org/10.1016/s0306-4530(98)00068-7

Bird, G., Silani, G., Brindley, R., White, S., Frith, U., \& Singer, T. (2010). Empathic brain responses in insula are modulated by levels of alexithymia but not autism. Brain, 133(5), 1515-1525. https://doi.org/10.1093/brain/awq060

Biundo, R., Weis, L., Facchini, S., Formento-Dojot, P., Vallelunga, A., Pilleri, M., Weintraub, D., \& Antonini, A. (2015). Patterns of cortical thickness associated with impulse control disorders in Parkinson's disease. Movement Disorders, 30(5), 688-695. https://doi.org/10.1002/mds.26154

Blair, R. J. R. (2001). ADVANCES IN NEUROPSYCHIATRY: Neurocognitive models of aggression, the antisocial personality disorders, and psychopathy. Journal of Neurology, Neurosurgery \& Psychiatry, 71(6), 727-731. https://doi.org/10.1136/jnnp.71.6.727

Blair, R. J. R. (2005). Responding to the emotions of others: Dissociating forms of empathy through the study of typical and psychiatric populations. Consciousness and Cognition, 14(4), 698-718. 
https://doi.org/10.1016/j.concog.2005.06.004

Blakemore, S.-J. (2008). The social brain in adolescence. Nature Reviews Neuroscience, 9(4), 267-277. https://doi.org/10.1038/nrn2353

Blakemore, S.-J., \& Choudhury, S. (2006). Development of the adolescent brain: implications for executive function and social cognition. Journal of Child Psychology and Psychiatry, 47(3-4), 296-312. https://doi.org/10.1111/j.1469-7610.2006.01611.x

Bogg, T., \& Lasecki, L. (2015). Reliable gains? Evidence for substantially underpowered designs in studies of working memory training transfer to fluid intelligence. In Frontiers in Psychology (Vol. 6, Issue JAN, p. 589). https://doi.org/10.3389/fpsyg.2015.00589

Borkovec, T. D., Robinson, E., Pruzinsky, T., \& DePree, J. A. (1983). Preliminary exploration of worry: Some characteristics and processes. Behaviour Research and Therapy, 21(1), 9-16. https://doi.org/10.1016/00057967(83)90121-3

Bornstein, M. H., \& Gardner, H. (1986). Frames of Mind: The Theory of Multiple Intelligences. In Journal of Aesthetic Education (Vol. 20, Issue 2). Basic Books. https://doi.org/10.2307/3332707

Botvinick, M. M., Cohen, J. D., \& Carter, C. S. (2004). Conflict monitoring and anterior cingulate cortex: An update. Trends in Cognitive Sciences, 8(12), 539-546. https://doi.org/10.1016/j.tics.2004.10.003

Brackett, M. A., Mayer, J. D., \& Warner, R. M. (2004). Emotional intelligence and its relation to everyday behaviour. Personality and Individual Differences, 36(6), 1387-1402. https://doi.org/10.1016/S0191-8869(03)00236-8

Brackett, M. A., Rivers, S. E., Reyes, M. R., \& Salovey, P. (2012). Enhancing academic performance and social and emotional competence with the RULER feeling words curriculum. Learning and Individual Differences, 22(2), 218-224. https://doi.org/10.1016/j.lindif.2010.10.002

Brannick, M. T., Wahi, M. M., Arce, M., Johnson, H. A., Nazian, S., \& Goldin, S. B. (2009). Comparison of trait and ability measures of emotional intelligence in medical students. Medical Education, 43(11), 1062-1068. https://doi.org/10.1111/j.1365-2923.2009.03430.x

Brody, L. R. (1997). Gender and emotion: Beyond stereotypes. Journal of Social Issues, 53(2), 369-393. https://doi.org/10.1111/j.1540-4560.1997.tb02448.x

Brons, L. L. (2015). Othering, an analysis. Transcience, a Journal of Global Studies, 6(1).

Brown, J. M. (2013). A sex difference in location-based inhibition-of-return. Personality and Individual Differences, 54(6), 721-725. https://doi.org/10.1016/j.paid.2012.11.029

Burgess, P. W., Veitch, E., de Lacy Costello, A., \& Shallice, T. (2000). The cognitive and neuroanatomical correlates of multitasking. Neuropsychologia, 38(6), 848-863. https://doi.org/10.1016/s0028-3932(99)00134-7

Caballero, A., Granberg, R., \& Tseng, K. Y. (2016). Mechanisms contributing to prefrontal cortex maturation during adolescence. Neuroscience and 
Biobehavioral Reviews, 70, 4-12.

https://doi.org/10.1016/j.neubiorev.2016.05.013

Cacioppo, J. T., Berntson, G. G., Sheridan, J. F., \& McClintock, M. K. (2000).

Multilevel integrative analyses of human behavior: Social neuroscience and the complementing nature of social and biological approaches.

Psychological Bulletin, 126(6), 829-843. https://doi.org/10.1037/00332909.126.6.829

Calhoun, V. D., Adali, T., Pearlson, G. D., \& Pekar, J. J. (2001). A method for making group inferences from functional MRI data using independent component analysis. Human Brain Mapping, 14(3), 140-151. https://doi.org/10.1002/hbm.1048

Cameron, Cunningham, W., Saunders, B., \& Inzlicht, M. (2017). The ends of empathy: Constructing empathy from value-based choice. https://doi.org/10.31234/osf.io/d99bp

Cameron, O. G. (2001). Interoception: The inside story - A model for psychosomatic processes. Psychosomatic Medicine, 63(5), 697-710. https://doi.org/10.1097/00006842-200109000-00001

Carlson, S. M., Mandell, D. J., \& Williams, L. (2004). Executive function and theory of mind: Stability and prediction from ages 2 to 3 . Developmental Psychology, 40(6), 1105-1122. https://doi.org/10.1037/0012-1649.40.6.1105

Carney, D. R., \& Harrigan, J. A. (2003). It takes one to know one: Interpersonal sensitivity is related to accurate assessments of others' interpersonal sensitivity. Emotion, 3(2), 194-200. https://doi.org/10.1037/15283542.3.2.194

Carr, L., lacoboni, M., Dubeau, M.-C., Mazziotta, J. C., \& Lenzi, G. L. (2003). Neural mechanisms of empathy in humans: A relay from neural systems for imitation to limbic areas. Proceedings of the National Academy of Sciences, 100(9), 5497-5502. https://doi.org/10.1073/pnas.0935845100

Catani, M., \& Thiebaut de Schotten, M. (2008). A diffusion tensor imaging tractography atlas for virtual in vivo dissections. Cortex, 44(8), 1105-1132. https://doi.org/10.1016/j.cortex.2008.05.004

Cauda, F., D’Agata, F., Sacco, K., Duca, S., Geminiani, G., \& Vercelli, A. (2011). Functional connectivity of the insula in the resting brain. Neurolmage, 55(1), 8-23. https://doi.org/10.1016/j.neuroimage.2010.11.049

Cerami, C., Dodich, A., Canessa, N., Crespi, C., Marcone, A., Cortese, F., Chierchia, G., Scola, E., Falini, A., \& Cappa, S. F. (2014). Neural correlates of empathic impairment in the behavioral variant of frontotemporal dementia. Alzheimer's \& Dementia, 10(6), 827-834. https://doi.org/10.1016/j.jalz.2014.01.005

Chan, R. C. K., Shum, D., Toulopoulou, T., \& Chen, E. Y. H. (2008). Assessment of executive functions: Review of instruments and identification of critical issues. Archives of Clinical Neuropsychology, 23(2), 201-216. https://doi.org/10.1016/j.acn.2007.08.010

Charoensukmongkol, P. (2014). Benefits of Mindfulness Meditation on Emotional 
Intelligence, General Self-Efficacy, and Perceived Stress: Evidence from Thailand. Journal of Spirituality in Mental Health, 16(3), 171-192. https://doi.org/10.1080/19349637.2014.925364

Chhatwal, J., \& Lane, R. D. (2016). A cognitive-developmental model of emotional awareness and its application to the practice of psychotherapy. In Psychodynamic Psychiatry (Vol. 44, Issue 2, pp. 305-325). Guilford Publications. https://doi.org/10.1521/pdps.2016.44.2.305

Christov-Moore, L., Reggente, N., Douglas, P. K., Feusner, J. D., \& lacoboni, M. (2020). Predicting Empathy From Resting State Brain Connectivity: A Multivariate Approach. In Frontiers in Integrative Neuroscience (Vol. 14, p. 3). https://doi.org/10.3389/fnint.2020.00003

Christov-Moore, L., Simpson, E. A., Coudé, G., Grigaityte, K., lacoboni, M., \& Ferrari, P. F. (2014). Empathy: Gender effects in brain and behavior. Neuroscience and Biobehavioral Reviews, 46(P4), 604-627. https://doi.org/10.1016/j.neubiorev.2014.09.001

Chu, L.-C. (2010). The benefits of meditation vis-à-vis emotional intelligence, perceived stress and negative mental health. Stress and Health, 26(2), 169180. https://doi.org/https://doi.org/10.1002/smi.1289

Cilia, R., Cho, S. S., van Eimeren, T., Marotta, G., Siri, C., Ko, J. H., Pellecchia, G., Pezzoli, G., Antonini, A., \& Strafella, A. P. (2011). Pathological gambling in patients with Parkinson's disease is associated with fronto-striatal disconnection: A path modeling analysis. Movement Disorders, 26(2), 225233. $\mathrm{https}: / /$ doi.org/10.1002/mds.23480

Clark, C. A. C., Pritchard, V. E., \& Woodward, L. J. (2010). Preschool executive functioning abilities predict early mathematics achievement. Developmental Psychology, 46(5), 1176-1191. https://doi.org/10.1037/a0019672

Cocchi, L., Zalesky, A., Fornito, A., \& Mattingley, J. B. (2013). Dynamic cooperation and competition between brain systems during cognitive control. Trends in Cognitive Sciences, 17(10), 493-501. https://doi.org/10.1016/j.tics.2013.08.006

Compare, A., Zarbo, C., Shonin, E., Van Gordon, W., \& Marconi, C. (2014). Emotional regulation and depression: A potential mediator between heart and mind. Cardiovascular Psychiatry and Neurology, 2014, 324374. https://doi.org/10.1155/2014/324374

Cooke, P. S., Nanjappa, M. K., Ko, C., Prins, G. S., \& Hess, R. A. (2017). Estrogens in Male Physiology. Physiological Reviews, 97(3), 995-1043. https://doi.org/10.1152/physrev.00018.2016

Cooney, R. E., Joormann, J., Eugène, F., Dennis, E. L., \& Gotlib, I. H. (2010). Neural correlates of rumination in depression. Cognitive, Affective, \& Behavioral Neuroscience, 10(4), 470-478. https://doi.org/10.3758/cabn.10.4.470

Cooper, A., \& Petrides, K. V. (2010). A psychometric analysis of the Trait Emotional Intelligence Questionnaire-Short Form (TEIQue-SF) using item response theory. Journal of Personality Assessment, 92(5), 449-457. 
https://doi.org/10.1080/00223891.2010.497426

Corbetta, M., \& Shulman, G. L. (2002). Control of goal-directed and stimulusdriven attention in the brain. Nature Reviews Neuroscience, 3(3), 201-215. https://doi.org/10.1038/nrn755

Cordes, D., Haughton, V. M., Arfanakis, K., Carew, J. D., Turski, P. A., Moritz, C. H., Quigley, M. A., \& Meyerand, M. E. (2001). Frequencies contributing to functional connectivity in the cerebral cortex in "resting-state" data. American Journal of Neuroradiology, 22(7), 1326-1333.

Core R Team. (2019). A Language and Environment for Statistical Computing. In $R$ Foundation for Statistical Computing (Vol. 2, p. https://www.R-project.org). R Foundation for Statistical Computing. http://www.r-project.org Cox, C. L., Uddin, L. Q., Di martino, A., Castellanos, F. X., Milham, M. P., \& Kelly, C. (2012). The balance between feeling and knowing: Affective and cognitive empathy are reflected in the brain's intrinsic functional dynamics. Social Cognitive and Affective Neuroscience, 7(6), 727-737. https://doi.org/10.1093/scan/nsr051

Craig, A. D. (2002). How do you feel? Interoception: the sense of the physiological condition of the body. Nature Reviews Neuroscience, 3(8), 655-666. https://doi.org/10.1038/nrn894

Craig, A. D. (2009). How do you feel - now? The anterior insula and human awareness. Nature Reviews Neuroscience, 10(1), 59-70. https://doi.org/10.1038/nrn2555

Critchley, H. D. (2005). Neural mechanisms of autonomic, affective, and cognitive integration. Journal of Comparative Neurology, 493(1), 154-166. https://doi.org/10.1002/cne.20749

Critchley, H. D., \& Garfinkel, S. N. (2017). Interoception and emotion. Current Opinion in Psychology, 17, 7-14. https://doi.org/10.1016/j.copsyc.2017.04.020

Critchley, H. D., Wiens, S., Rotshtein, P., Öhman, A., \& Dolan, R. J. (2004). Neural systems supporting interoceptive awareness. Nature Neuroscience, 7(2), 189-195. https://doi.org/10.1038/nn1176

Crocker, L. D., Heller, W., Warren, S. L., O'Hare, A. J., Infantolino, Z. P., \& Miller, G. A. (2013). Relationships among cognition, emotion, and motivation: Implications for intervention and neuroplasticity in psychopathology. In Frontiers in Human Neuroscience (Vol. 7, Issue MAY, p. 261). https://doi.org/10.3389/fnhum.2013.00261

Crone, E. A. (2009). Executive functions in adolescence: inferences from brain and behavior. Developmental Science, 12(6), 825-830. https://doi.org/10.1111/j.1467-7687.2009.00918.x

D’Esposito, M., Postle, B. R., Ballard, D., \& Lease, J. (1999). Maintenance versus manipulation of information held in working memory: An event-related fMRI study. Brain and Cognition, 41(1), 66-86. https://doi.org/10.1006/brcg.1999.1096

Dai, R., Huang, Z., Tu, H., Wang, L., Tanabe, S., Weng, X., He, S., \& Li, D. 
(2016). Interplay between heightened temporal variability of spontaneous brain activity and task-evoked hyperactivation in the blind. Frontiers in Human Neuroscience, 10(DEC2016), 632. https://doi.org/10.3389/fnhum.2016.00632

Dale, A. M., \& Sereno, M. I. (1993). Improved Localizadon of Cortical Activity by Combining EEG and MEG with MRI Cortical Surface Reconstruction: A Linear Approach. Journal of Cognitive Neuroscience, 5(2), 162-176. https://doi.org/10.1162/jocn.1993.5.2.162

Dalili, M. N., Penton-Voak, I. S., Harmer, C. J., \& Munafò, M. R. (2015). Metaanalysis of emotion recognition deficits in major depressive disorder. Psychological Medicine, 45(6), 1135-1144. https://doi.org/10.1017/S0033291714002591

Damaraju, E., Allen, E. A., Belger, A., Ford, J. M., McEwen, S., Mathalon, D. H., Mueller, B. A., Pearlson, G. D., Potkin, S. G., Preda, A., Turner, J. A., Vaidya, J. G., Van Erp, T. G., \& Calhoun, V. D. (2014). Dynamic functional connectivity analysis reveals transient states of dysconnectivity in schizophrenia. Neurolmage: Clinical, 5, 298-308. https://doi.org/10.1016/j.nicl.2014.07.003

Damasio, A. R. (2005). Descartes' error: emotion, reason, and the human brain. Damasio, A. R., Grabowski, T. J., Bechara, A., Damasio, H., Ponto, L. L. B., Parvizi, J., \& Hichwa, R. D. (2000). Subcortical and cortical brain activity during the feeling of self-generated emotions. Nature Neuroscience, 3(10), 1049-1056. https://doi.org/10.1038/79871

Dan, R., Canetti, L., Keadan, T., Segman, R., Weinstock, M., Bonne, O., Reuveni, I., \& Goelman, G. (2019). Sex differences during emotion processing are dependent on the menstrual cycle phase. Psychoneuroendocrinology, 100, 85-95. https://doi.org/10.1016/j.psyneuen.2018.09.032

Davidson, R. J. (1998). Affective Style and Affective Disorders: Perspectives from Affective Neuroscience. Cognition and Emotion, 12(3), 307-330. https://doi.org/10.1080/026999398379628

Davidson, R. J., Putnam, K. M., \& Larson, C. L. (2000). Dysfunction in the neural circuitry of emotion regulation - A possible prelude to violence. Science, 289(5479), 591-594. https://doi.org/10.1126/science.289.5479.591

Davies, M., Stankov, L., \& Roberts, R. D. (1998). Emotional intelligence: in search of an elusive construct. In Journal of personality and social psychology (Vol. 75, Issue 4, pp. 989-1015). American Psychological Association. https://doi.org/10.1037/0022-3514.75.4.989

Davis. (1983). Measuring individual differences in empathy: Evidence for a multidimensional approach. Journal of Personality and Social Psychology, 44(1), 113-126. https://doi.org/10.1037/0022-3514.44.1.113

Davis, M. H. (1980). A multidimensional approach to individual differences in empathy. JSAS Catalog of Selected Documents in Psychology, 10, 85. http://www.uv.es/ friasnav/Davis_1980.pdf 
Day, A. L., \& Carroll, S. A. (2004). Using an ability-based measure of emotional intelligence to predict individual performance, group performance, and group citizenship behaviours. Personality and Individual Differences, 36(6), 14431458. https://doi.org/10.1016/S0191-8869(03)00240-X

De Waal, F. B. M., \& Preston, S. D. (2017). Mammalian empathy: Behavioural manifestations and neural basis. In Nature Reviews Neuroscience (Vol. 18, Issue 8, pp. 498-509). Nature Publishing Group. https://doi.org/10.1038/nrn.2017.72

Decety, J., Chen, C., Harenski, C., \& Kiehl, K. A. (2013). An fMRI study of affective perspective taking in individuals with psychopathy: Imagining another in pain does not evoke empathy. Frontiers in Human Neuroscience, 7(SEP), 489. https://doi.org/10.3389/fnhum.2013.00489

Decety, J., Chen, C., Harenski, C. L., \& Kiehl, K. A. (2015). Socioemotional processing of morally-laden behavior and their consequences on others in forensic psychopaths. Human Brain Mapping, 36(6), 2015-2026. https://doi.org/10.1002/hbm.22752

Decety, J., \& Jackson, P. L. (2004). The functional architecture of human empathy. Behavioral and Cognitive Neuroscience Reviews, 3(2), 71-100. https://doi.org/10.1177/1534582304267187

Decety, J., \& Lamm, C. (2006). Human Empathy Through the Lens of Social Neuroscience. The Scientific World JOURNAL, 6, 1146-1163. https://doi.org/10.1100/tsw.2006.221

Decety, J., \& Moriguchi, Y. (2007). The empathic brain and its dysfunction in psychiatric populations: Implications for intervention across different clinical conditions. In BioPsychoSocial Medicine (Vol. 1). https://doi.org/10.1186/1751-0759-1-22

Decety, J., \& Sommerville, J. A. (2003). Shared representations between self and other: A social cognitive neuroscience view. Trends in Cognitive Sciences, 7(12), 527-533. https://doi.org/10.1016/j.tics.2003.10.004

Denkova, E., Nomi, J. S., Uddin, L. Q., \& Jha, A. P. (2019). Dynamic brain network configurations during rest and an attention task with frequent occurrence of mind wandering. Human Brain Mapping, 40(15), 4564-4576. https://doi.org/10.1002/hbm.24721

Depue, B. E., Orr, J. M., Smolker, H. R., Naaz, F., \& Banich, M. T. (2016). The Organization of Right Prefrontal Networks Reveals Common Mechanisms of Inhibitory Regulation Across Cognitive, Emotional, and Motor Processes. Cerebral Cortex, 26(4), 1634-1646. https://doi.org/10.1093/cercor/bhu324

Derksen, J., Kramer, I., \& Katzko, M. (2002). Does a self-report measure for emotional intelligence assess something different than general intelligence? Personality and Individual Differences, 32(1), 37-48. https://doi.org/10.1016/S0191-8869(01)00004-6

Desikan, R. S., Ségonne, F., Fischl, B., Quinn, B. T., Dickerson, B. C., Blacker, D., Buckner, R. L., Dale, A. M., Maguire, R. P., Hyman, B. T., Albert, M. S., \& Killiany, R. J. (2006). An automated labeling system for subdividing the 
human cerebral cortex on MRI scans into gyral based regions of interest. Neurolmage, 31(3), 968-980.

https://doi.org/10.1016/j.neuroimage.2006.01.021

Dimaggio, G., Lysaker, P. H., Carcione, A., Nicolò, G., \& Semerari, A. (2008). Know yourself and you shall know the other... to a certain extent: Multiple paths of influence of self-reflection on mindreading. Consciousness and Cognition, 17(3), 778-789. https://doi.org/10.1016/j.concog.2008.02.005

Domes, G., Schulze, L., Böttger, M., Grossmann, A., Hauenstein, K., Wirtz, P. H., Heinrichs, M., \& Herpertz, S. C. (2010). The neural correlates of sex differences in emotional reactivity and emotion regulation. Human Brain Mapping, 31(5), 758-769. https://doi.org/10.1002/hbm.20903

Donders, F. C. (1969). On the speed of mental processes. Acta Psychologica, 30(C), 412-431. https://doi.org/10.1016/0001-6918(69)90065-1

Dorris, L., Espie, C. A. E., Knott, F., \& Salt, J. (2004). Mind-reading difficulties in the siblings of people with Asperger's syndrome: Evidence for a genetic influence in the abnormal development of a specific cognitive domain. Journal of Child Psychology and Psychiatry and Allied Disciplines, 45(2), 412-418. https://doi.org/10.1111/j.1469-7610.2004.00232.x

Doucet. (2019). Consensual Atlas of REsting-state Networks (CAREN).

Doucet, Naveau, M., Petit, L., Delcroix, N., Zago, L., Crivello, F., Jobard, G., Tzourio-Mazoyer, N., Mazoyer, B., Mellet, E., \& Joliot, M. (2011). Brain activity at rest: A multiscale hierarchical functional organization. Journal of Neurophysiology, 105(6), 2753-2763. https://doi.org/10.1152/jn.00895.2010

Doucet, Rasgon, N., Mcewen, B., Micali, N., \& Frangou, S. (2018). BrainFunctionalAtlas.zip.

Ducharme, S., Albaugh, M. D., Nguyen, T.-V., Hudziak, J. J., Mateos-Pérez, J. M., Labbe, A., Evans, A. C., Karama, S., \& Group, B. D. C. (2015).

Trajectories of cortical surface area and cortical volume maturation in normal brain development. Data in Brief, 5, 929-938. https://doi.org/10.1016/j.dib.2015.10.044

Dunn, B. D., Galton, H. C., Morgan, R., Evans, D., Oliver, C., Meyer, M., Cusack, R., Lawrence, A. D., \& Dalgleish, T. (2010). Listening to your heart: How interoception shapes emotion experience and intuitive decision making. Psychological Science, 21(12), 1835-1844. https://doi.org/10.1177/0956797610389191

Dvash, J., \& Shamay-Tsoory, S. G. (2014). Theory of Mind and Empathy as Multidimensional Constructs. Topics in Language Disorders, 34(4), 282-295. https://doi.org/10.1097/tld.0000000000000040

Elton, A., \& Gao, W. (2014). Divergent task-dependent functional connectivity of executive control and salience networks. Cortex, 51(1), 56-66. https://doi.org/10.1016/j.cortex.2013.10.012

Epskamp, S., Cramer, A. O. J., Waldorp, L. J., Schmittmann, V. D., \& Borsboom, D. (2012). Qgraph: Network visualizations of relationships in psychometric data. Journal of Statistical Software, 48(1), 1-18. 
https://doi.org/10.18637/jss.v048.i04

Ernst, J., Northoff, G., Böker, H., Seifritz, E., \& Grimm, S. (2013). Interoceptive awareness enhances neural activity during empathy. Human Brain Mapping, 34(7), 1615-1624. https://doi.org/10.1002/hbm.22014

Etkin, A., Büchel, C., \& Gross, J. J. (2015). The neural bases of emotion regulation. Nature Reviews Neuroscience, 16(11), 693-700. https://doi.org/10.1038/nrn4044

Etkin, A., Egner, T., Peraza, D. M., Kandel, E. R., \& Hirsch, J. (2006). Resolving Emotional Conflict: A Role for the Rostral Anterior Cingulate Cortex in Modulating Activity in the Amygdala. Neuron, 51(6), 871-882. https://doi.org/10.1016/j.neuron.2006.07.029

Etkin, A., \& Wager, T. D. (2007). Functional Neuroimaging of Anxiety: A MetaAnalysis of Emotional Processing in PTSD, Social Anxiety Disorder, and Specific Phobia. American Journal of Psychiatry, 164(10), 1476-1488. https://doi.org/10.1176/appi.ajp.2007.07030504

Evans, A. C. (2006). The NIH MRI study of normal brain development. Neurolmage, 30(1), 184-202. https://doi.org/10.1016/j.neuroimage.2005.09.068

Fabes, R. A., \& Martin, C. L. (1991). Gender and Age Stereotypes of Emotionality. Personality and Social Psychology Bulletin, 17(5), 532-540. https://doi.org/10.1177/0146167291175008

Faisal, A. A., Selen, L. P. J., \& Wolpert, D. M. (2008). Noise in the nervous system. Nature Reviews Neuroscience, 9(4), 292-303. https://doi.org/10.1038/nrn2258

Fan, J., Flombaum, J. I., McCandliss, B. D., Thomas, K. M., \& Posner, M. I. (2003). Cognitive and brain consequences of conflict. Neurolmage, 18(1), 42-57. https://doi.org/10.1006/nimg.2002.1319

Fan, Y., Duncan, N. W., de Greck, M., \& Northoff, G. (2011). Is there a core neural network in empathy? An fMRI based quantitative meta-analysis. In Neuroscience and Biobehavioral Reviews (Vol. 35, Issue 3, pp. 903-911). Pergamon. https://doi.org/10.1016/j.neubiorev.2010.10.009

Faridi, N., Karama, S., Burgaleta, M., White, M. T., Evans, A. C., Fonov, V., Collins, D. L., \& Waber, D. P. (2015). Neuroanatomical correlates of behavioral rating versus performance measures of working memory in typically developing children and adolescents. Neuropsychology, 29(1), 8291. https://doi.org/10.1037/neu0000079

Feinstein, J. S., Stein, M. B., \& Paulus, M. P. (2006). Anterior insula reactivity during certain decisions is associated with neuroticism. Social Cognitive and Affective Neuroscience, 1(2), 136-142. https://doi.org/10.1093/scan/nsl016

Finn, E. S., Shen, X., Scheinost, D., Rosenberg, M. D., Huang, J., Chun, M. M., Papademetris, X., \& Constable, R. T. (2015). Functional connectome fingerprinting: Identifying individuals using patterns of brain connectivity. Nature Neuroscience, 18(11), 1664-1671. https://doi.org/10.1038/nn.4135

Fischer, A. H. (1993). Sex Differences in Emotionality: Fact or Stereotype? 
Feminism \& Psychology, 3(3), 303-318.

https://doi.org/10.1177/0959353593033002

Fischl, B., Liu, A., \& Dale, A. M. (2001). Automated manifold surgery:

constructing geometrically accurate and topologically correct models of the human cerebral cortex. IEEE Transactions on Medical Imaging, 20(1), 7080. https://doi.org/10.1109/42.906426

Fischl, B, \& Dale, A. M. (2000). Measuring the thickness of the human cerebral cortex from magnetic resonance images. Proceedings of the National Academy of Sciences of the United States of America, 97(20), 1105011055. https://doi.org/10.1073/pnas.200033797

Fischl, Bruce, Sereno, M. I., \& Dale, A. M. (1999). Cortical Surface-Based Analysis: II: Inflation, Flattening, and a Surface-Based Coordinate System. Neurolmage, 9(2), 195-207. https://doi.org/10.1006/nimg.1998.0396

Fischl, Bruce, van der Kouwe, A., Destrieux, C., Halgren, E., Ségonne, F., Salat, D. H., Busa, E., Seidman, L. J., Goldstein, J., Kennedy, D., Caviness, V., Makris, N., Rosen, B., \& Dale, A. M. (2004). Automatically parcellating the human cerebral cortex. Cerebral Cortex (New York, N.Y. : 1991), 14(1), $11-$ 22. https://doi.org/10.1093/CERCOR/BHG087

Fonagy, P., \& Luyten, P. (2009). A developmental, mentalization-based approach to the understanding and treatment of borderline personality disorder.

Development and Psychopathology, 21(4), 1355-1381. https://doi.org/10.1017/S0954579409990198

Fox, R. (2005). Cataloging our information architecture. OCLC Systems and Services, 21(1), 23-29. https://doi.org/10.1108/10650750510578118

Frank, D. W., Dewitt, M., Hudgens-Haney, M., Schaeffer, D. J., Ball, B. H., Schwarz, N. F., Hussein, A. A., Smart, L. M., \& Sabatinelli, D. (2014). Emotion regulation: Quantitative meta-analysis of functional activation and deactivation. Neuroscience and Biobehavioral Reviews, 45, 202-211. https://doi.org/10.1016/j.neubiorev.2014.06.010

Fregni, F., Boggio, P. S., Nitsche, M., Bermpohl, F., Antal, A., Feredoes, E., Marcolin, M. A., Rigonatti, S. P., Silva, M. T. A., Paulus, W., \& PascualLeone, A. (2005). Anodal transcranial direct current stimulation of prefrontal cortex enhances working memory. Experimental Brain Research, 166(1), 23-30. https://doi.org/10.1007/s00221-005-2334-6

Fresco, D. M., Frankel, A. N., Mennin, D. S., Turk, C. L., \& Heimberg, R. G. (2002). No Title. Cognitive Therapy and Research, 26(2), 179-188. https://doi.org/10.1023/a:1014517718949

Frith, C. D., \& Frith, U. (2006). The Neural Basis of Mentalizing. Neuron, 50(4), 531-534. https://doi.org/10.1016/j.neuron.2006.05.001

Frith, U., Morton, J., \& Leslie, A. M. (1991). The cognitive basis of a biological disorder: autism. Trends in Neurosciences, 14(10), 433-438. https://doi.org/10.1016/0166-2236(91)90041-R

Gambin, M., \& Sharp, C. (2016). The Differential Relations Between Empathy and Internalizing and Externalizing Symptoms in Inpatient Adolescents. 
Child Psychiatry and Human Development, 47(6), 966-974.

https://doi.org/10.1007/s10578-016-0625-8

Gambin, M., \& Sharp, C. (2018). Relations between empathy and anxiety dimensions in inpatient adolescents. Anxiety, Stress, \& Coping, 31(4), 447458. https://doi.org/10.1080/10615806.2018.1475868

Gannon, N., \& Ranzijn, R. (2005). Does emotional intelligence predict unique variance in life satisfaction beyond IQ and personality? Personality and Individual Differences, 38(6), 1353-1364. https://doi.org/10.1016/j.paid.2004.09.001

Garcia-Larrea, L. (2012). The posterior insular-opercular region and the search of a primary cortex for pain. Neurophysiologie Clinique, 42(5), 299-313. https://doi.org/10.1016/j.neucli.2012.06.001

Gard, M. G., \& Kring, A. M. (2007). Sex differences in the time course of emotion. In Emotion (Vol. 7, Issue 2, pp. 429-437). American Psychological Association. https://doi.org/10.1037/1528-3542.7.2.429

Garic, D., Broce, I., Graziano, P., Mattfeld, A., \& Dick, A. S. (2018). Laterality of the frontal aslant tract ( FAT ) explains externalizing behaviors through its association with executive function. Developmental Science, 22(2). https://doi.org/10.1111/desc.12744

Garnefski, N., Teerds, J., Kraaij, V., Legerstee, J., \& van den Kommer, T. (2004). Cognitive emotion regulation strategies and depressive symptoms: Differences between males and females. Personality and Individual Differences, 36(2), 267-276. https://doi.org/10.1016/S0191-8869(03)000837

Garrett, D. D., Kovacevic, N., McIntosh, A. R., \& Grady, C. L. (2010). Blood oxygen level-dependent signal variability is more than just noise. Journal of Neuroscience, 30(14), 4914-4921. https://doi.org/10.1523/JNEUROSCI.5166-09.2010

Garrett, D. D., Kovacevic, N., McIntosh, A. R., \& Grady, C. L. (2013). The modulation of BOLD variability between cognitive states varies by age and processing speed. Cerebral Cortex, 23(3), 684-693. https://doi.org/10.1093/cercor/bhs055

Garrett, D. D., Samanez-Larkin, G. R., MacDonald, S. W. S., Lindenberger, U., Mclntosh, A. R., \& Grady, C. L. (2013). Moment-to-moment brain signal variability: A next frontier in human brain mapping? Neuroscience and Biobehavioral Reviews, 37(4), 610-624. https://doi.org/10.1016/j.neubiorev.2013.02.015

Gaut, G., Li, X., Turner, B., Cunningham, W. A., Lu, Z. L., \& Steyvers, M. (2018). Predicting task and subject differences with functional connectivity and bold variability. ArXiv, 9(6), 451-463.

Gavazzi, G., Orsolini, S., Rossi, A., Bianchi, A., Bartolini, E., Nicolai, E., Soricelli, A., Aiello, M., Diciotti, S., Viggiano, M. P., \& Mascalchi, M. (2017). Alexithymic trait is associated with right IFG and pre-SMA activation in nonemotional response inhibition in healthy subjects. Neuroscience Letters, 658, 
150-154. https://doi.org/10.1016/j.neulet.2017.08.031

Gawronski, I., \& Privette, G. (1997). Empathy and reactive depression.

Psychological Reports, 80(3), 1043-1049.

https://doi.org/10.2466/pr0.1997.80.3.1043

Genova, H. M., DeLuca, J., Chiaravalloti, N., \& Wylie, G. (2013). The relationship between executive functioning, processing speed, and white matter integrity in multiple sclerosis. Journal of Clinical and Experimental Neuropsychology, 35(6), 631-641. https://doi.org/10.1080/13803395.2013.806649

Giedd, J. N. (2004). Structural magnetic resonance imaging of the adolescent brain. Annals of the New York Academy of Sciences, 1021(1), 77-85. https://doi.org/10.1196/annals.1308.009

Giedd, J. N., Blumenthal, J., Jeffries, N. O., Castellanos, F. X., Liu, H., Zijdenbos, A., Paus, T., Evans, A. C., \& Rapoport, J. L. (1999). Brain development during childhood and adolescence: a longitudinal MRI study. Nature Neuroscience, 2(10), 861-863. https://doi.org/10.1038/13158

Gillies, G. E., \& McArthur, S. (2010). Estrogen actions in the brain and the basis for differential action in men and women: a case for sex-specific medicines. Pharmacological Reviews, 62(2), 155-198. https://doi.org/10.1124/pr.109.002071

Giombini, L. (2015). Handbook of emotion regulation. In Advances in Eating Disorders (Vol. 3, Issue 1). https://doi.org/10.1080/21662630.2014.953321

Giorgio, A., Watkins, K. E., Chadwick, M., James, S., Winmill, L., Douaud, G., De Stefano, N., Matthews, P. M., Smith, S. M., Johansen-Berg, H., \& James, A. C. (2010). Longitudinal changes in grey and white matter during adolescence. Neurolmage, 49(1), 94-103.

https://doi.org/10.1016/j.neuroimage.2009.08.003

Goleman, D. (1995). Emotional Intelligence. Random House.

Gonzalez-Castillo, J., Hoy, C. W., Handwerker, D. A., Robinson, M. E., Buchanan, L. C., Saad, Z. S., \& Bandettini, P. A. (2015). Tracking ongoing cognition in individuals using brief, whole-brain functional connectivity patterns. Proceedings of the National Academy of Sciences of the United States of America, 112(28), 8762-8767. https://doi.org/10.1073/pnas.1501242112

Good, T. J., Villafuerte, J., Ryan, J. D., Grady, C. L., \& Barense, M. D. (2020). Resting state bold variability of the posterior medial temporal lobe correlates with cognitive performance in older adults with and without risk for cognitive decline. ENeuro, 7(3), ENEURO.0290-19.2020. https://doi.org/10.1523/ENEURO.0290-19.2020

Gordon, E. M., Laumann, T. O., Adeyemo, B., Huckins, J. F., Kelley, W. M., \& Petersen, S. E. (2016). Generation and Evaluation of a Cortical Area Parcellation from Resting-State Correlations. Cerebral Cortex, 26(1), 288303. https://doi.org/10.1093/cercor/bhu239

Gray, M. A., Harrison, N. A., Wiens, S., \& Critchley, H. D. (2007). Modulation of emotional appraisal by false physiological feedback during fMRI. PLOS ONE, 
2(6), e546. https://doi.org/10.1371/journal.pone.0000546

Green, M. J., Cahill, C. M., \& Malhi, G. S. (2007). The cognitive and neurophysiological basis of emotion dysregulation in bipolar disorder. Journal of Affective Disorders, 103(1-3), 29-42. https://doi.org/10.1016/j.jad.2007.01.024

Greenberg, D. L., Rice, H. J., Cooper, J. J., Cabeza, R., Rubin, D. C., \& LaBar, K. S. (2005). Co-activation of the amygdala, hippocampus and inferior frontal gyrus during autobiographical memory retrieval. Neuropsychologia, 43(5), 659-674. https://doi.org/10.1016/j.neuropsychologia.2004.09.002

Greicius, M. D., Krasnow, B., Reiss, A. L., \& Menon, V. (2003). Functional connectivity in the resting brain: A network analysis of the default mode hypothesis. Proceedings of the National Academy of Sciences of the United States of America, 100(1), 253-258.

https://doi.org/10.1073/pnas.0135058100

Grewal, D., Brackett, M., \& Salovey, P. (2006). Emotional intelligence and the self-regulation of affect.

Gross, James J. (1998). The emerging field of emotion regulation: An integrative review. Review of General Psychology, 2(3), 271-299.

https://doi.org/10.1037/1089-2680.2.3.271

Gross, James J. (2002). Emotion regulation: Affective, cognitive, and social consequences. Psychophysiology. https://doi.org/10.1017/S0048577201393198

Gross, James J. (2015). Emotion Regulation: Current Status and Future Prospects. Psychological Inquiry, 26(1), 1-26. https://doi.org/10.1080/1047840X.2014.940781

Gross, James J., \& John, O. P. (2003). Individual Differences in Two Emotion Regulation Processes: Implications for Affect, Relationships, and WellBeing. Journal of Personality and Social Psychology, 85(2), 348-362. https://doi.org/10.1037/0022-3514.85.2.348

Gross, James J., \& Muñoz, R. F. (1995). Emotion Regulation and Mental Health. Clinical Psychology: Science and Practice, 2(2), 151-164. https://doi.org/10.1111/j.1468-2850.1995.tb00036.x

Grossman, M., \& Wood, W. (1993). Sex Differences in Intensity of Emotional Experience: A Social Role Interpretation. Journal of Personality and Social Psychology, 65(5), 1010-1022. https://doi.org/10.1037/0022-3514.65.5.1010

Gur, R. C., Gunning-Dixon, F., Bilker, W. B., \& Gur, R. E. (2002). Sex differences in temporo-limbic and frontal brain volumes of healthy adults. Cerebral Cortex, 12(9), 998-1003. https://doi.org/10.1093/cercor/12.9.998

Gurney, E. (1884). "What is an emotion?" In Mind: Vol. os-IX (Issue 35). https://doi.org/10.1093/mind/os-IX.35.421

Hagelskamp, C., Brackett, M. A., Rivers, S. E., \& Salovey, P. (2013). Improving Classroom Quality with The RULER Approach to Social and Emotional Learning: Proximal and Distal Outcomes. American Journal of Community Psychology, 51(3-4), 530-543. https://doi.org/10.1007/s10464-013-9570-x 
Hampshire, A., Thompson, R., Duncan, J., \& Owen, A. M. (2009). Selective tuning of the right inferior frontal gyrus during target detection. Cognitive, Affective and Behavioral Neuroscience, 9(1), 103-112. https://doi.org/10.3758/CABN.9.1.103

Hampson, E., \& Morley, E. E. (2013). Estradiol concentrations and working memory performance in women of reproductive age.

Psychoneuroendocrinology, 38(12), 2897-2904. https://doi.org/10.1016/j.psyneuen.2013.07.020

Hampson, E., van Anders, S. M., \& Mullin, L. I. (2006). A female advantage in the recognition of emotional facial expressions: test of an evolutionary hypothesis. Evolution and Human Behavior, 27(6), 401-416. https://doi.org/10.1016/j.evolhumbehav.2006.05.002

Hansen, S. (2011). Inhibitory control and empathy-related personality traits: Sexlinked associations. Brain and Cognition, 76(3), 364-368.

Harkness, K., Sabbagh, M., Jacobson, J., Chowdrey, N., \& Chen, T. (2005). Enhanced accuracy of mental state decoding in dysphoric college students. Cognition \& Emotion, 19(7), 999-1025. https://doi.org/10.1080/02699930541000110

Harmon-Jones, E., Lueck, L., Fearn, M., \& Harmon-Jones, C. (2006). The Effect of Personal Relevance and Approach-Related Action Expectation on Relative Left Frontal Cortical Activity. Psychological Science, 17(5), 434440. https://doi.org/10.1111/j.1467-9280.2006.01724.x

Hatfield, E., Cacioppo, J. T., \& Rapson, R. L. (1993). Emotional Contagion. Current Directions in Psychological Science, 2(3), 96-100. https://doi.org/10.1111/1467-8721.ep10770953

Hayes, A. F. (2012). PROCESS: A versatile computational tool for observed variable moderation, mediation, and conditional process modeling. Manuscript Submitted for Publication, January, 1-39. http://www.afhayes.com/public/process2012.pdf

Healey, M. L., \& Grossman, M. (2018). Cognitive and affective perspectivetaking: Evidence for shared and dissociable anatomical substrates. In Frontiers in Neurology (Vol. 9, Issue JUN, p. 491). https://doi.org/10.3389/fneur.2018.00491

Hecke, W. Van, Nagels, G., Leemans, A., Vandervliet, E., Sijbers, J., \& Parizel, P. M. (2010). Correlation of cognitive dysfunction and diffusion tensor MRI measures in patients with mild and moderate multiple sclerosis. Journal of Magnetic Resonance Imaging, 31(6), 1492-1498. https://doi.org/10.1002/jmri.22198

Hemmati, T., Mills, J. F., \& Kroner, D. G. (2004). The validity of the Bar-On emotional intelligence quotient in an offender population. Personality and Individual Differences, 37(4), 695-706. https://doi.org/10.1016/j.paid.2003.10.003

Herting, M. M., Gautam, P., Spielberg, J. M., Kan, E., Dahl, R. E., \& Sowell, E. R. (2014). The role of testosterone and estradiol in brain volume changes 
across adolescence: a longitudinal structural MRI study. Human Brain Mapping, 35(11), 5633-5645. https://doi.org/10.1002/hbm.22575

Herting, M. M., Maxwell, E. C., Irvine, C., \& Nagel, B. J. (2012). The impact of sex, puberty, and hormones on white matter microstructure in adolescents. Cerebral Cortex (New York, N.Y. : 1991), 22(9), 1979-1992. https://doi.org/10.1093/cercor/bhr246

Hess, U., Senecal, S., Kirouac, G., Herrera, P., Philippot, P., \& Kleck, R. E. (2000). Emotional expressivity in men and women: Stereotypes and selfperceptions. Cognition and Emotion, 14(5), 609-642. https://doi.org/10.1080/02699930050117648

Hidalgo-Lopez, E., \& Pletzer, B. (2017). Interactive Effects of Dopamine Baseline Levels and Cycle Phase on Executive Functions: The Role of Progesterone. Frontiers in Neuroscience, 11, 403. https://doi.org/10.3389/fnins.2017.00403

Higo, T., Mars, R. B., Boorman, E. D., Buch, E. R., \& Rushworth, M. F. S. (2011). Distributed and causal influence of frontal operculum in task control. Proceedings of the National Academy of Sciences of the United States of America, 108(10), 4230-4235. https://doi.org/10.1073/pnas.1013361108

Himberg, J., Hyvärinen, A., \& Esposito, F. (2004). Validating the independent components of neuroimaging time series via clustering and visualization. Neurolmage, 22(3), 1214-1222. https://doi.org/10.1016/j.neuroimage.2004.03.027

Holmes, M. M., Wide, J. K., \& Galea, L. A. M. (2002). Low levels of estradiol facilitate, whereas high levels of estradiol impair, working memory performance on the radial arm maze. Behavioral Neuroscience, 116(5), 928-934. https://doi.org/10.1037/0735-7044.116.5.928

Hooker, C. I., Verosky, S. C., Germine, L. T., Knight, R. T., \& D’Esposito, M. (2008). Mentalizing about emotion and its relationship to empathy. Social Cognitive and Affective Neuroscience, 3(3), 204-217. https://doi.org/10.1093/scan/nsn019

Horley, K., Williams, L. M., Gonsalvez, C., \& Gordon, E. (2004). Face to face: visual scanpath evidence for abnormal processing of facial expressions in social phobia. Psychiatry Research, 127(1-2), 43-53.

https://doi.org/10.1016/j.psychres.2004.02.016

Hornak, J., Bramham, J., Rolls, E. T., Morris, R. G., O'Doherty, J., Bullock, P. R., \& Polkey, C. E. (2003). Changes in emotion after circumscribed surgical lesions of the orbitofrontal and cingulate cortices. Brain, 126(7), 1691-1712. https://doi.org/10.1093/brain/awg168

Howard, L. R., Kumaran, D., Ólafsdóttir, H. F., \& Spiers, H. J. (2013). Dissociation between Dorsal and Ventral Posterior Parietal Cortical Responses to Incidental Changes in Natural Scenes. PLOS ONE, 8(7), e67988-e67988. https://doi.org/10.1371/journal.pone.0067988

Hutchison, R. M., Womelsdorf, T., Allen, E. A., Bandettini, P. A., Calhoun, V. D., Corbetta, M., Della Penna, S., Duyn, J. H., Glover, G. H., Gonzalez-Castillo, J., Handwerker, D. A., Keilholz, S., Kiviniemi, V., Leopold, D. A., de 
Pasquale, F., Sporns, O., Walter, M., \& Chang, C. (2013). Dynamic functional connectivity: Promise, issues, and interpretations. Neurolmage, 80, 360-378. https://doi.org/10.1016/j.neuroimage.2013.05.079

Hyde, D. C., Aparicio Betancourt, M., \& Simon, C. E. (2015). Human temporalparietal junction spontaneously tracks others' beliefs: A functional nearinfrared spectroscopy study. Human Brain Mapping, 36(12), 4831-4846. https://doi.org/10.1002/hbm.22953

lacoboni, M. (2005). Understanding others: Imitation, language, empathy. Perspectives on Imitation: From Cognitive Neuroscience to Social Science, 1, 77-99.

Ickes, W., \& Hodges, S. D. (2013). Empathic Accuracy in Close Relationships. In Oxford Handbooks Online. Oxford University Press. https://doi.org/10.1093/oxfordhb/9780195398694.013.0016

Iriki, A. (2006). The neural origins and implications of imitation, mirror neurons and tool use. Current Opinion in Neurobiology, 16(6), 660-667. https://doi.org/10.1016/j.conb.2006.10.008

Isen, A. M., Rosenzweig, A. S., \& Young, M. J. (1991). The influence of positive affect on clinical problem solving. Medical Decision Making, 11(3), 221-227. https://doi.org/10.1177/0272989X9101100313

Jabbi, M., \& Keysers, C. (2008). Inferior Frontal Gyrus Activity Triggers Anterior Insula Response to Emotional Facial Expressions. In Emotion (Vol. 8, Issue 6, pp. 775-780). American Psychological Association. https://doi.org/10.1037/a0014194

Jabbi, M., Swart, M., \& Keysers, C. (2007). Empathy for positive and negative emotions in the gustatory cortex. Neurolmage, 34(4), 1744-1753. https://doi.org/10.1016/j.neuroimage.2006.10.032

Jackson, P. L., Meltzoff, A. N., \& Decety, J. (2005). How do we perceive the pain of others? A window into the neural processes involved in empathy. Neurolmage, 24(3), 771-779. https://doi.org/10.1016/j.neuroimage.2004.09.006

Johnson, M. (2001). The Feeling of What Happens: Body and Emotion in the Making of Consciousness (review). In The Journal of Speculative Philosophy (Vol. 15, Issue 4). Houghton Mifflin Harcourt. https://doi.org/10.1353/jsp.2001.0038

Joireman, J. A., III, L. P., \& Hammersla, J. (2002). Empathy and the SelfAbsorption Paradox: Support for the Distinction Between Self-Rumination and Self-Reflection. Self and Identity, 1(1), 53-65. https://doi.org/10.1080/152988602317232803

Joormann, J., \& Stanton, C. H. (2016). Examining emotion regulation in depression: A review and future directions. In Behaviour Research and Therapy (Vol. 86, pp. 35-49). Elsevier Ltd. https://doi.org/10.1016/j.brat.2016.07.007

Joseph, R. M., \& Tager-Flusberg, H. (2004). The relationship of theory of mind and executive functions to symptom type and severity in children with 
autism. Development and Psychopathology, 16(1), 137-155.

https://doi.org/10.1017/S095457940404444X

Kafetsios, K. (2004). Attachment and emotional intelligence abilities across the life course. Personality and Individual Differences, 37(1), 129-145. https://doi.org/10.1016/j.paid.2003.08.006

Kalisch, R. (2009). The functional neuroanatomy of reappraisal: Time matters. Neuroscience \& Biobehavioral Reviews, 33(8), 1215-1226. https://doi.org/https://doi.org/10.1016/j.neubiorev.2009.06.003

Karbach, J., \& Unger, K. (2014). Executive control training from middle childhood to adolescence. Frontiers in Psychology, 5, 390. https://doi.org/10.3389/fpsyg.2014.00390

Kastner, S., De Weerd, P., Desimone, R., \& Ungerleider, L. G. (1998). Mechanisms of directed attention in the human extrastriate cortex as revealed by functional MRI. Science, 282(5386), 108-111. https://doi.org/10.1126/science.282.5386.108

Kaźmierczak, M., Pastwa-Wojciechowska, B., \& Błazek, M. (2013). A multidimensional model of empathy, and the occurrence of personality disorders and stress in social settings. Acta Neuropsychologica, 11(2), 113125. https://doi.org/10.5604/17307503.1073470

Keidar, D. (2015). Emotional Intelligence and Education. Studia Edukacyjne, 37, 327-348. https://doi.org/10.14746/se.2015.37.19

Kessler, R. C., McGonagle, K. A., Swartz, M., Blazer, D. G., \& Nelson, C. B. (1993). Sex and depression in the National Comorbidity Survey I: Lifetime prevalence, chronicity and recurrence. Journal of Affective Disorders, 29(23), 85-96. https://doi.org/10.1016/0165-0327(93)90026-G

Keysers, C., Wicker, B., Gazzola, V., Anton, J. L., Fogassi, L., \& Gallese, V. (2004). A touching sight: SII/PV activation during the observation and experience of touch. Neuron, 42(2), 335-346. https://doi.org/10.1016/S08966273(04)00156-4

Khalsa, S. S., Adolphs, R., Cameron, O. G., Critchley, H. D., Davenport, P. W., Feinstein, J. S., Feusner, J. D., Garfinkel, S. N., Lane, R. D., Mehling, W. E., Meuret, A. E., Nemeroff, C. B., Oppenheimer, S., Petzschner, F. H., Pollatos, O., Rhudy, J. L., Schramm, L. P., Simmons, W. K., Stein, M. B., ... Zucker, N. (2018). Interoception and Mental Health: A Roadmap. Biological Psychiatry: Cognitive Neuroscience and Neuroimaging, 3(6), 501-513. https://doi.org/10.1016/j.bpsc.2017.12.004

Kharitonova, M., Martin, R. E., Gabrieli, J. D. E., \& Sheridan, M. A. (2013). Cortical gray-matter thinning is associated with age-related improvements on executive function tasks. Developmental Cognitive Neuroscience, 6, 61-71. https://doi.org/10.1016/j.dcn.2013.07.002

Killgore, W. D. S., \& Yurgelun-Todd, D. A. (2007). The right-hemisphere and valence hypotheses: Could they both be right (and sometimes left)? Social Cognitive and Affective Neuroscience, 2(3), 240-250.

https://doi.org/10.1093/scan/nsm020 
Kim, J. A., Szatmari, P., Bryson, S. E., Streiner, D. L., \& Wilson, F. J. (2000). The prevalence of anxiety and mood problems among children with autism and Asperger syndrome. Autism, 4(2), 117-132. https://doi.org/10.1177/1362361300004002002

Kleckner, I. R., Zhang, J., Touroutoglou, A., Chanes, L., Xia, C., Simmons, W. K., Quigley, K. S., Dickerson, B. C., \& Feldman Barrett, L. (2017). Evidence for a large-scale brain system supporting allostasis and interoception in humans. Nature Human Behaviour, 1(5), 69. https://doi.org/10.1038/s41562017-0069

Kohn, N., Eickhoff, S. B., Scheller, M., Laird, A. R., Fox, P. T., \& Habel, U. (2014). Neural network of cognitive emotion regulation - An ALE metaanalysis and MACM analysis. Neurolmage, 87, 345-355. https://doi.org/10.1016/j.neuroimage.2013.11.001

Kong, F., Zhen, Z., Li, J., Huang, L., Wang, X., Song, Y., \& Liu, J. (2014). Sexrelated neuroanatomical basis of emotion regulation ability. PLOS ONE, 9(5), e97071-e97071. https://doi.org/10.1371/journal.pone.0097071

Konishi, S., Nakajima, K., Uchida, I., Kikyo, H., Kameyama, M., \& Miyashita, Y. (1999). Common inhibitory mechanism in human inferior prefrontal cortex revealed by event-related functional MRI. Brain, 122(5), 981-991. https://doi.org/10.1093/brain/122.5.981

Konstantareas, M. M., \& Stewart, K. (2006). Affect regulation and temperament in children with Autism Spectrum Disorder. Journal of Autism and Developmental Disorders, 36(2), 143-154. https://doi.org/10.1007/s10803005-0051-4

Kovács, Á. M., Kühn, S., Gergely, G., Csibra, G., \& Brass, M. (2014). Are all beliefs equal? Implicit belief attributions recruiting core brain regions of theory of mind. PLOS ONE, 9(9). https://doi.org/10.1371/journal.pone.0106558

Kraus, M. F., Susmaras, T., Caughlin, B. P., Walker, C. J., Sweeney, J. A., \& Little, D. M. (2007). White matter integrity and cognition in chronic traumatic brain injury: a diffusion tensor imaging study. Brain, 130(10), 2508-2519. https://doi.org/10.1093/brain/awm216

Kring, A. M., \& Gordon, A. H. (1998). Sex Differences in Emotion: Expression, Experience, and Physiology. In Journal of Personality and Social Psychology (Vol. 74, Issue 3, pp. 686-703). American Psychological Association. https://doi.org/10.1037/0022-3514.74.3.686

Kringelbach, M. L. (2005). The human orbitofrontal cortex: Linking reward to hedonic experience. Nature Reviews Neuroscience, 6(9), 691-702. https://doi.org/10.1038/nrn1747

Kross, E., Davidson, M., Weber, J., \& Ochsner, K. (2009). Coping with Emotions Past: The Neural Bases of Regulating Affect Associated with Negative Autobiographical Memories. Biological Psychiatry, 65(5), 361-366. https://doi.org/10.1016/j.biopsych.2008.10.019

Kuehn, E., Mueller, K., Lohmann, G., \& Schuetz-Bosbach, S. (2016). 
Interoceptive awareness changes the posterior insula functional connectivity profile. Brain Structure and Function, 221(3), 1555-1571.

https://doi.org/10.1007/s00429-015-0989-8

Kuhn, C., Johnson, M., Thomae, A., Luo, B., Simon, S. A., Zhou, G., \& Walker, Q. D. (2010). The emergence of gonadal hormone influences on dopaminergic function during puberty. Hormones and Behavior, 58(1), 122137. https://doi.org/10.1016/j.yhbeh.2009.10.015

Kumral, D., Şansal, F., Cesnaite, E., Mahjoory, K., Al, E., Gaebler, M., Nikulin, V. V., \& Villringer, A. (2020). BOLD and EEG signal variability at rest differently relate to aging in the human brain. Neurolmage, 207, 116373. https://doi.org/10.1016/j.neuroimage.2019.116373

Laird, J. D., Alibozak, T., Davainis, D., Deignan, K., Fontanella, K., Hong, J., Levy, B., \& Pacheco, C. (1994). Individual differences in the effects of spontaneous mimicry on emotional contagion. Motivation and Emotion, 18(3), 231-247. https://doi.org/10.1007/BF02254830

Lamm, C., Batson, C. D., \& Decety, J. (2007). The neural substrate of human empathy: Effects of perspective-taking and cognitive appraisal. Journal of Cognitive Neuroscience, 19(1), 42-58.

https://doi.org/10.1162/jocn.2007.19.1.42

Lane, R. D. (2008). Neural substrates of implicit and explicit emotional processes: A unifying framework for psychosomatic medicine.

Psychosomatic Medicine, 70(2), 214-231. https://doi.org/10.1097/PSY.0b013e3181647e44

Lang, P., Bradley, M., \& Cuthbert, B. (2005). International Affective Picture System (IAPS): Digitized Photographs, Instruction Manual and Affective Ratings. Technical Report A-6. In 2005.

Lau, H., Rogers, R. D., \& Passingham, R. E. (2006). Dissociating response selection and conflict in the medial frontal surface. Neurolmage, 29(2), 446451. https://doi.org/10.1016/j.neuroimage.2005.07.050

Lawrence, E. J., Shaw, P., Giampietro, V. P., Surguladze, S., Brammer, M. J., \& David, A. S. (2006). The role of "shared representations" in social perception and empathy: An fMRI study. Neurolmage, 29(4), 1173-1184.

https://doi.org/10.1016/j.neuroimage.2005.09.001

Lazarus, R. S., \& Alfert, E. (1964). Short-circuiting of threat by experimentally altering cognitive appraisal. Journal of Abnormal and Social Psychology, 69(2), 195-205. https://doi.org/10.1037/h0044635

Leach, L. S., Christensen, H., Mackinnon, A. J., Windsor, T. D., \& Butterworth, P. (2008). Gender differences in depression and anxiety across the adult lifespan: The role of psychosocial mediators. Social Psychiatry and Psychiatric Epidemiology, 43(12), 983-998. https://doi.org/10.1007/s00127008-0388-z

Lee, E. (1920). Intelligence and its use. Harper's Magazine, 227-235.

Lee, K. H., \& Siegle, G. J. (2012). Common and distinct brain networks underlying explicit emotional evaluation: A meta-analytic study. Social 
Cognitive and Affective Neuroscience, 7(5), 521-534.

https://doi.org/10.1093/scan/nsp001

Lee, T. W., Dolan, R. J., \& Critchley, H. D. (2008). Controlling emotional expression: Behavioral and neural correlates of nonimitative emotional responses. Cerebral Cortex, 18(1), 104-113. https://doi.org/10.1093/cercor/bhm035

Leiberg, S., \& Anders, S. (2006). Chapter 23 The multiple facets of empathy: a survey of theory and evidence. In S. Anders, G. Ende, M. Junghofer, J. Kissler, \& D. B. T.-P. in B. R. Wildgruber (Eds.), Progress in Brain Research (Vol. 156, pp. 419-440). Elsevier. https://doi.org/10.1016/S00796123(06)56023-6

Leigh, R., Oishi, K., Hsu, J., Lindquist, M., Gottesman, R. F., Jarso, S., Crainiceanu, C., Mori, S., \& Hillis, A. E. (2013). Acute lesions that impair affective empathy. Brain, 136(8), 2539-2549. https://doi.org/10.1093/brain/awt177

Lenroot, R. K., \& Giedd, J. N. (2010). Sex differences in the adolescent brain. Brain and Cognition, 72(1), 46-55. https://doi.org/10.1016/j.bandc.2009.10.008

Lewis, J. G., McGill, H., Patton, V. M., \& Elder, P. A. (2002). Caution on the use of saliva measurements to monitor absorption of progesterone from transdermal creams in postmenopausal women. Maturitas, 41(1), 1-6. https://doi.org/10.1016/s0378-5122(01)00250-x

Li, H., Yuan, J., \& Lin, C. (2008). The neural mechanism underlying the female advantage in identifying negative emotions: An event-related potential study. Neurolmage, 40(4), 1921-1929. https://doi.org/10.1016/j.neuroimage.2008.01.033

Li, X., Lu, Z. L., D’Argembeau, A., Ng, M., \& Bechara, A. (2010). The lowa Gambling Task in fMRI images. Human Brain Mapping, 31(3), 410-423. https://doi.org/10.1002/hbm.20875

Liberzon, I., Duval, E., \& Javanbakht, A. (2015). Neural circuits in anxiety and stress disorders: a\&nbsp;focused review. Therapeutics and Clinical Risk Management, 115. https://doi.org/10.2147/tcrm.s48528

Lieberman, M. D., \& Eisenberger, N. I. (2009). Neuroscience: Pains and pleasures of social life. Science, 323(5916), 890-891. https://doi.org/10.1126/science. 1170008

Linden, D. E. J. (2007). The working memory networks of the human brain. Neuroscientist, 13(3), 257-267. https://doi.org/10.1177/1073858406298480

Lindström, B. R., \& Bohlin, G. (2011). Emotion processing facilitates working memory performance. Cognition and Emotion, 25(7), 1196-1204. https://doi.org/10.1080/02699931.2010.527703

Linhartová, P., Látalová, A., Kóša, B., Kašpárek, T., Schmahl, C., \& Paret, C. (2019). fMRI neurofeedback in emotion regulation: A literature review. Neurolmage, 193, 75-92. https://doi.org/10.1016/j.neuroimage.2019.03.011 Lopes, P. N., Salovey, P., Côté, S., \& Beers, M. (2005). Emotion regulation 
abilities and the quality of social interaction. In R. E. Petty (Ed.), Emotion (Vol. 5, Issue 1, pp. 113-118). American Psychological Association. https://doi.org/10.1037/1528-3542.5.1.113

Losh, M., Adolphs, R., Poe, M. D., Couture, S., Penn, D., Baranek, G. T., \& Piven, J. (2009). Neuropsychological profile of autism and the broad autism phenotype. Archives of General Psychiatry, 66(5), 518-526. https://doi.org/10.1001/archgenpsychiatry.2009.34

Luebbe, A. M., Bell, D. J., Allwood, M. A., Swenson, L. P., \& Early, M. C. (2010). Social Information Processing in Children: Specific Relations to Anxiety, Depression, and Affect. Journal of Clinical Child \& Adolescent Psychology, 39(3), 386-399. https://doi.org/10.1080/15374411003691685

Lungu, O., Potvin, S., Tikàsz, A., \& Mendrek, A. (2015). Sex differences in effective fronto-limbic connectivity during negative emotion processing. Psychoneuroendocrinology, 62, 180-188. https://doi.org/10.1016/j.psyneuen.2015.08.012

Lutkenhoff, E. S., Rosenberg, M., Chiang, J., Zhang, K., Pickard, J. D., Owen, A. M., \& Monti, M. M. (2014). Optimized Brain Extraction for Pathological Brains (optiBET). PLoS ONE, 9(12), e115551. https://doi.org/10.1371/journal.pone.0115551

MacDonald, S. W. S., Nyberg, L., \& Bäckman, L. (2006). Intra-individual variability in behavior: links to brain structure, neurotransmission and neuronal activity. Trends in Neurosciences, 29(8), 474-480. https://doi.org/10.1016/j.tins.2006.06.011

MacKinnon, D. P., Krull, J. L., \& Lockwood, C. M. (2000). Equivalence of the mediation, confounding and suppression effect. Prevention Science, 1(4), 173-181. https://doi.org/10.1023/A:1026595011371

Mahler K. (2015). Interoception: The Eighth Sensory System: Practical Solutions for Improving Self-Regulation, Self-Awareness and Social Understanding. AAPC Publishing.

Mahone, E. M., Martin, R., Kates, W. R., Hay, T., \& Horská, A. (2009). Neuroimaging correlates of parent ratings of working memory in typically developing children. Journal of the International Neuropsychological Society : JINS, 15(1), 31-41. https://doi.org/10.1017/S1355617708090164

Mak, A. K. Y., Hu, Z. guo, Zhang, J. X. X., Xiao, Z., \& Lee, T. M. C. (2009). Sexrelated differences in neural activity during emotion regulation.

Neuropsychologia, 47(13), 2900-2908. https://doi.org/10.1016/j.neuropsychologia.2009.06.017

Mandell, D., Siegle, G. J., Shutt, L., Feldmiller, J., \& Thase, M. E. (2014). Neural substrates of trait ruminations in depression. Journal of Abnormal Psychology, 123(1), 35-48. https://doi.org/10.1037/a0035834

Mar, R. A. (2011). The neural bases of social cognition and story comprehension. Annual Review of Psychology, 62, 103-134. https://doi.org/10.1146/annurev-psych-120709-145406

Mauss, I. B., Bunge, S. A., \& Gross, J. J. (2007). Automatic Emotion Regulation. 
Social and Personality Psychology Compass, 1(1), 146-167. https://doi.org/10.1111/j.1751-9004.2007.00005.x

Mayer, J. D. (2004). What is Emotional Intelligence? What is Emotional Intelligence? What Is Emotional Intelligence? Contents. Emotional Development and Emotional Intelligence: Implications for Educators. https://scholars.unh.edu/personality_lab

Mayer, J. D., Caruso, D. R., \& Salovey, P. (1999). Emotional intelligence meets traditional standards for an intelligence. Intelligence, 27(4), 267-298. https://doi.org/10.1016/S0160-2896(99)00016-1

Mayer, J. D., Caruso, D. R., \& Salovey, P. (2016). The Ability Model of Emotional Intelligence: Principles and Updates. Emotion Review, 8(4), 290-300. https://doi.org/10.1177/1754073916639667

Mayer, J. D., Salovey, P., Caruso, D. R., \& Sitarenios, G. (2003). Measuring Emotional Intelligence with the MSCEIT V2.0. In Emotion (Vol. 3, Issue 1, pp. 97-105). American Psychological Association. https://doi.org/10.1037/1528-3542.3.1.97

McCarthy, M. M. (2008). Estradiol and the developing brain. Physiological Reviews, 88(1), 91-124. https://doi.org/10.1152/physrev.00010.2007

McEvoy, P. M., Watson, H., Watkins, E. R., \& Nathan, P. (2013). The relationship between worry, rumination, and comorbidity: Evidence for repetitive negative thinking as a transdiagnostic construct. Journal of Affective Disorders, 151(1), 313-320. https://doi.org/10.1016/j.jad.2013.06.014

McIntosh, A. R., Kovacevic, N., \& Itier, R. J. (2008). Increased brain signal variability accompanies lower behavioral variability in development. PLoS Computational Biology, 4(7), e1000106-e1000106. https://doi.org/10.1371/journal.pcbi.1000106

McLaughlin, K. A., \& Nolen-Hoeksema, S. (2011). Rumination as a transdiagnostic factor in depression and anxiety. Behaviour Research and Therapy, 49(3), 186-193. https://doi.org/10.1016/j.brat.2010.12.006

McLean, C. P., Asnaani, A., Litz, B. T., \& Hofmann, S. G. (2011). Gender differences in anxiety disorders: Prevalence, course of illness, comorbidity and burden of illness. Journal of Psychiatric Research, 45(8), 1027-1035. https://doi.org/10.1016/j.jpsychires.2011.03.006

McRae, K., Ochsner, K. N., Mauss, I. B., Gabrieli, J. J. D., \& Gross, J. J. (2008). Gender differences in emotion regulation: An fMRI study of cognitive reappraisal. Group Processes and Intergroup Relations, 11(2), 143-162. https://doi.org/10.1177/1368430207088035

Mehling, W. E., Price, C., Daubenmier, J. J., Acree, M., Bartmess, E., \& Stewart, A. (2012). The Multidimensional Assessment of Interoceptive Awareness (MAIA). PLOS ONE, 7(11), e48230. https://doi.org/10.1371/journal.pone.0048230

Menon, \& Uddin. (2010). Saliency, switching, attention and control: a network model of insula function. Brain Structure \& Function, 214(5-6), 655-667. https://doi.org/10.1007/s00429-010-0262-0 
Menon, V. (2015). Salience Network. Brain Mapping: An Encyclopedic

Reference, 2, 597-611. https://doi.org/10.1016/B978-0-12-397025-1.00052$\mathrm{X}$

Mesulam, M. -Marchsel. (1981). A cortical network for directed attention and unilateral neglect. Annals of Neurology, 10(4), 309-325. https://doi.org/10.1002/ana.410100402

Meyer, T. J., Miller, M. L., Metzger, R. L., \& Borkovec, T. D. (1990). Development and validation of the penn state worry questionnaire. Behaviour Research and Therapy, 28(6), 487-495. https://doi.org/10.1016/0005-7967(90)90135-6

Mišíc, B., Vakorin, V. A., Paus, T., \& Mclntosh, A. R. (2011). Functional embedding predicts the variability of neural activity. Frontiers in Systems Neuroscience, 5(NOVEMBER 2011), 90. https://doi.org/10.3389/fnsys.2011.00090

Mueller, S. C. (2011). The influence of emotion on cognitive control: Relevance for development and adolescent psychopathology. In Frontiers in Psychology (Vol. 2, Issue NOV, p. 327). https://doi.org/10.3389/fpsyg.2011.00327

Mullins-Nelson, J. L., Salekin, R. T., \& Leistico, A. M. R. (2006). Psychopathy, empathy, and perspective -taking ability in a community sample: Implications for the successful psychopathy concept. International Journal of Forensic Mental Health, 5(2), 133-149. https://doi.org/10.1080/14999013.2006.10471238

Nagy, Z., Westerberg, H., \& Klingberg, T. (2004). Maturation of White Matter is Associated with the Development of Cognitive Functions during Childhood. Journal of Cognitive Neuroscience, 16(7), 1227-1233.

https://doi.org/10.1162/0898929041920441

Naughtin, C. K., Horne, K., Schneider, D., Venini, D., York, A., \& Dux, P. E. (2017). Do implicit and explicit belief processing share neural substrates? Human Brain Mapping, 38(9), 4760-4772. https://doi.org/10.1002/hbm.23700

Nejad, A. B., Fossati, P., \& Lemogne, C. (2013). Self-Referential Processing, Rumination, and Cortical Midline Structures in Major Depression. Frontiers in Human Neuroscience, 7. https://doi.org/10.3389/fnhum.2013.00666

Nelson, S. M., Dosenbach, N. U. F., Cohen, A. L., Wheeler, M. E., Schlaggar, B. L., \& Petersen, S. E. (2010). Role of the anterior insula in task-level control and focal attention. Brain Structure \& Function, 214(5-6), 669-680. https://doi.org/10.1007/s00429-010-0260-2

Nguyen, T.-V., McCracken, J., Ducharme, S., Botteron, K. N., Mahabir, M., Johnson, W., Israel, M., Evans, A. C., Karama, S., \& Group, B. D. C. (2013). Testosterone-related cortical maturation across childhood and adolescence. Cerebral Cortex (New York, N.Y. : 1991), 23(6), 1424-1432. https://doi.org/10.1093/cercor/bhs125

Nichols, T. E., \& Holmes, A. P. (2001). Nonparametric permutation tests for functional neuroimaging: A primer with examples. Human Brain Mapping, 
15(1), 1-25. https://doi.org/10.1002/hbm.1058

Nieto-Castanon, A. (2014). NITRC: CONN : functional connectivity toolbox: File Release Notes and Changelog.

https://www.nitrc.org/frs/shownotes.php?release_id=2733

Nieto-Castanon, A. (2015). NITRC: CONN : functional connectivity toolbox: RE: Conn-Preprocessing-Band-Pass.

https://www.nitrc.org/forum/message.php?msg_id=12691

Nieto-Castanon, A. (2020). NITRC: CONN : ICA variability calculation method.

https://www.nitrc.org/forum/forum.php?set=custom\&forum_id=1144\&style=n

ested\&max_rows $=50$

Nolen-Hoeksema, S. (1998). The Other End of the Continuum: The Costs of

Rumination. Psychological Inquiry, 9(3), 216-219.

https://doi.org/10.1207/s15327965pli0903_5

Nolen-Hoeksema, S. (2001). Gender differences in depression. In Current Directions in Psychological Science (Vol. 10, Issue 5, pp. 173-176).

Blackwell Publishing Inc. https://doi.org/10.1111/1467-8721.00142

Nolen-Hoeksema, S. (2012). Emotion regulation and psychopathology: The role of gender. Annual Review of Clinical Psychology, 8(1), 161-187.

https://doi.org/10.1146/annurev-clinpsy-032511-143109

Nomi, J. S., Bolt, T. S., Chiemeka Ezie, C. E., Uddin, L. Q., \& Heller, A. S. (2017). Moment-to-moment BOLD signal variability reflects regional changes in neural flexibility across the lifespan. Journal of Neuroscience, 37(22), 5539-5548. https://doi.org/10.1523/JNEUROSCI.3408-16.2017

Northoff, G., Heinzel, A., de Greck, M., Bermpohl, F., Dobrowolny, H., \& Panksepp, J. (2006). Self-referential processing in our brain-A meta-analysis of imaging studies on the self. Neurolmage, 31(1), 440-457. https://doi.org/10.1016/j.neuroimage.2005.12.002

O'Driscoll, C., Laing, J., \& Mason, O. (2014). Cognitive emotion regulation strategies, alexithymia and dissociation in schizophrenia, a review and metaanalysis. Clinical Psychology Review, 34(6), 482-495.

https://doi.org/10.1016/j.cpr.2014.07.002

Ochsner, K. N., Bunge, S. A., Gross, J. J., \& Gabrieli, J. D. E. (2002). Rethinking feelings: An fMRI study of the cognitive regulation of emotion. Journal of Cognitive Neuroscience, 14(8), 1215-1229. https://doi.org/10.1162/089892902760807212

Ochsner, K. N., \& Gross, J. J. (2005). The cognitive control of emotion. Trends in Cognitive Sciences, 9(5), 242-249. https://doi.org/10.1016/j.tics.2005.03.010

Ochsner, K. N., Silvers, J. A., \& Buhle, J. T. (2012). Functional imaging studies of emotion regulation: a synthetic review and evolving model of the cognitive control of emotion. Annals of the New York Academy of Sciences, 1251(1), E1-E24. https://doi.org/10.1111/j.1749-6632.2012.06751.x

Ohira, H., Nomura, M., Ichikawa, N., Isowa, T., lidaka, T., Sato, A., Fukuyama, S., Nakajima, T., \& Yamada, J. (2006). Association of neural and physiological responses during voluntary emotion suppression. Neurolmage, 
29(3), 721-733. https://doi.org/10.1016/j.neuroimage.2005.08.047

Olatunji, B. O., Naragon-Gainey, K., \& Wolitzky-Taylor, K. B. (2013). Specificity of Rumination in Anxiety and Depression: A Multimodal Meta-Analysis. Clinical Psychology: Science and Practice, 20(3), 225-257. https://doi.org/10.1111/cpsp.12037

Ondobaka, S., Kilner, J., \& Friston, K. (2017). The role of interoceptive inference in theory of mind. Brain and Cognition, 112, 64-68. https://doi.org/10.1016/j.bandc.2015.08.002

Osaka, M., Yaoi, K., Minamoto, T., \& Osaka, N. (2013). When do negative and positive emotions modulate working memory performance? Scientific Reports, 3(1), 1375. https://doi.org/10.1038/srep01375

Owens, A. P., Allen, M., Ondobaka, S., \& Friston, K. J. (2018). Interoceptive inference: From computational neuroscience to clinic. Neuroscience and Biobehavioral Reviews, 90, 174-183. https://doi.org/10.1016/j.neubiorev.2018.04.017

Ozonoff, S., Pennington, B. F., \& Rogers, S. J. (1991). Executive Function Deficits in High-Functioning Autistic Individuals: Relationship to Theory of Mind. Journal of Child Psychology and Psychiatry, 32(7), 1081-1105. https://doi.org/10.1111/j.1469-7610.1991.tb00351.x

Pan, J., Zhan, L., Hu, C. L., Yang, J., Wang, C., Gu, L., Zhong, S., Huang, Y., Wu, Q., Xie, X., Chen, Q., Zhou, H., Huang, M., \& Wu, X. (2018). Emotion regulation and complex brain networks: Association between expressive suppression and efficiency in the fronto-parietal network and default-mode network. Frontiers in Human Neuroscience, 12. https://doi.org/10.3389/fnhum.2018.00070

Pardini, M., \& Nichelli, P. F. (2009). Age-related decline in mentalizing skills across adult life span. Experimental Aging Research, 35(1), 98-106. https://doi.org/10.1080/03610730802545259

Paulesu, E., Sambugaro, E., Torti, T., Danelli, L., Ferri, F., Scialfa, G., Sberna, M., Ruggiero, G. M., Bottini, G., \& Sassaroli, S. (2009). Neural correlates of worry in generalized anxiety disorder and in normal controls: a functional MRI study. Psychological Medicine, 40(1), 117-124. https://doi.org/10.1017/s0033291709005649

Paulus, M. P., \& Stein, M. B. (2006). An insular view of anxiety. Biological Psychiatry, 60(4), 383-387.

Paus, T. (2005). Mapping brain maturation and cognitive development during adolescence. Trends in Cognitive Sciences, 9(2), 60-68. https://doi.org/10.1016/j.tics.2004.12.008

Peiffer, C., Costes, N., Hervé, P., \& Garcia-Larrea, L. (2008). Relief of dyspnea involves a characteristic brain activation and a specific quality of sensation. American Journal of Respiratory and Critical Care Medicine, 177(4), 440449. https://doi.org/10.1164/rccm.200612-1774OC

Peña-Sarrionandia, A., Mikolajczak, M., \& Gross, J. J. (2015). Integrating emotion regulation and emotional intelligence traditions: A meta-analysis. 
Frontiers in Psychology, 6(FEB), 160.

https://doi.org/10.3389/fpsyg.2015.00160

Penny, W., Friston, K., Ashburner, J., Kiebel, S., \& Nichols, T. (2007). Statistical

Parametric Mapping: The Analysis of Functional Brain Images. In Statistical Parametric Mapping: The Analysis of Functional Brain Images. Elsevier Ltd. https://doi.org/10.1016/B978-0-12-372560-8.X5000-1

Peper, J. S., Brouwer, R. M., Schnack, H. G., van Baal, G. C., van Leeuwen, M., van den Berg, S. M., Delemarre-Van de Waal, H. A., Boomsma, D. I., Kahn, R. S., \& Hulshoff Pol, H. E. (2009). Sex steroids and brain structure in pubertal boys and girls. Psychoneuroendocrinology, 34(3), 332-342. https://doi.org/10.1016/j.psyneuen.2008.09.012

Peper, J. S., de Reus, M. A., van den Heuvel, M. P., \& Schutter, D. J. L. G. (2015). Short fused? associations between white matter connections, sex steroids, and aggression across adolescence. Human Brain Mapping, 36(3), 1043-1052. https://doi.org/10.1002/hbm.22684

Peper, J. S., van den Heuvel, M. P., Mandl, R. C. W., Pol, H. E. H., \& van Honk, J. (2011). Sex steroids and connectivity in the human brain: A review of neuroimaging studies. Psychoneuroendocrinology, 36(8), 1101-1113. https://doi.org/10.1016/j.psyneuen.2011.05.004

Pérez-Iglesias, R., Tordesillas-Gutiérrez, D., McGuire, P. K., Barker, G. J., RoizSantiañez, R., Mata, I., de Lucas, E. M., Rodríguez-Sánchez, J. M., AyesaArriola, R., Vazquez-Barquero, J. L., \& Crespo-Facorro, B. (2010). White Matter Integrity and Cognitive Impairment in First-Episode Psychosis. American Journal of Psychiatry, 167(4), 451-458. https://doi.org/10.1176/appi.ajp.2009.09050716

Perrett, D. I., Hietanen, J. K., Oram, M. W., \& Benson, P. J. (1992). Organization and functions of cells responsive to faces in the temporal cortex. Philosophical Transactions of the Royal Society of London. Series B, Biological Sciences, 335(1273), 23-30. https://doi.org/10.1098/rstb.1992.0003

Perrett, D. I., Rolls, E. T., \& Caan, W. (1982). Visual neurones responsive to faces in the monkey temporal cortex. Experimental Brain Research, 47(3), 329-342. https://doi.org/10.1007/BF00239352

Perrett, D. I., Smith, P. A. J., Potter, D. D., Mistlin, A. J., Head, A. S., Milner, A. D., \& Jeeves, M. A. (1985). Visual cells in the temporal cortex sensitive to face view and gaze direction. Proceedings of the Royal Society of London Biological Sciences, 223(1232), 293-317. https://doi.org/10.1098/rspb.1985.0003

Perry, D., Hendler, T., \& Shamay-Tsoory, S. G. (2011). Projecting memories: The role of the hippocampus in emotional mentalizing. Neurolmage, 54(2), 16691676. https://doi.org/10.1016/j.neuroimage.2010.08.057

Pessoa, L., Padmala, S., Kenzer, A., \& Bauer, A. (2012). Interactions between cognition and emotion during response inhibition. Emotion, 12(1), 192-197. https://doi.org/10.1037/a0024109 
Petrides, K. V., \& Furnham, A. (2000). On the dimensional structure of emotional intelligence. Personality and Individual Differences, 29(2), 313-320. https://doi.org/10.1016/S0191-8869(99)00195-6

Phillips, M. L., Ladouceur, C. D., \& Drevets, W. C. (2008). A neural model of voluntary and automatic emotion regulation: Implications for understanding the pathophysiology and neurodevelopment of bipolar disorder. Molecular Psychiatry, 13(9), 833-857. https://doi.org/10.1038/mp.2008.65

Phillips, Mary L., Drevets, W. C., Rauch, S. L., \& Lane, R. (2003). Neurobiology of emotion perception II: Implications for major psychiatric disorders. Biological Psychiatry, 54(5), 515-528. https://doi.org/10.1016/S00063223(03)00171-9

Picó-Pérez, M., Radua, J., Steward, T., Menchón, J. M., \& Soriano-Mas, C. (2017). Emotion regulation in mood and anxiety disorders: A meta-analysis of fMRI cognitive reappraisal studies. In Progress in NeuroPsychopharmacology and Biological Psychiatry (Vol. 79, pp. 96-104). Elsevier Inc. https://doi.org/10.1016/j.pnpbp.2017.06.001

Pizzagalli, D. A. (2011). Frontocingulate dysfunction in depression: Toward biomarkers of treatment response. Neuropsychopharmacology, 36(1), 183206. https://doi.org/10.1038/npp.2010.166

Plant, E. A., Hyde, J. S., Keltner, D., \& Devine, P. G. (2000). The gender stereotyping of emotions. Psychology of Women Quarterly, 24(1), 81-92. https://doi.org/10.1111/j.1471-6402.2000.tb01024.x

Poldrack, R. A. (2000). Imaging brain plasticity: Conceptual and methodological issues - A theoretical review. Neurolmage, 12(1), 1-13. https://doi.org/10.1006/nimg.2000.0596

Poldrack, R. A. (2011). Inferring mental states from neuroimaging data: From reverse inference to large-scale decoding. Neuron, 72(5), 692-697. https://doi.org/10.1016/j.neuron.2011.11.001

Pollatos, O., Gramann, K., \& Schandry, R. (2007). Neural systems connecting interoceptive awareness and feelings. Human Brain Mapping, 28(1), 9-18. https://doi.org/10.1002/hbm.20258

Pollatos, O., \& Schandry, R. (2008). Emotional processing and emotional memory are modulated by interoceptive awareness. Cognition and Emotion, 22(2), 272-287. https://doi.org/10.1080/02699930701357535

Powell, P. A. (2018). Individual differences in emotion regulation moderate the associations between empathy and affective distress. Motivation and Emotion, 42(4), 602-613. https://doi.org/10.1007/s11031-018-9684-4

Prasad, K. M., Upton, C. H., Nimgaonkar, V. L., \& Keshavan, M. S. (2015). Differential susceptibility of white matter tracts to inflammatory mediators in schizophrenia: an integrated DTI study. Schizophrenia Research, 161(1), 119-125. https://doi.org/10.1016/j.schres.2014.09.043

Preacher, K. J., \& Hayes, A. F. (2004). SPSS and SAS procedures for estimating indirect effects in simple mediation models. Behavior Research Methods, Instruments, and Computers, 36(4), 717-731. 
https://doi.org/10.3758/BF03206553

Preckel, K., Kanske, P., \& Singer, T. (2018). On the interaction of social affect and cognition: empathy, compassion and theory of mind. Current Opinion in Behavioral Sciences, 19, 1-6. https://doi.org/10.1016/j.cobeha.2017.07.010

Premack, D., \& Woodruff, G. (1978). Chimpanzee problem-solving: A test for comprehension. Science, 202(4367), 532-535.

https://doi.org/10.1126/science.705342

PRIBRAM, K. H. (1973). the Primate Frontal Cortex - Executive of the Brain. In Psychophysiology of the Frontal Lobes (pp. 293-314). Elsevier. https://doi.org/10.1016/b978-0-12-564340-5.50019-6

Pruim, R. H. R., Mennes, M., van Rooij, D., Llera, A., Buitelaar, J. K., \& Beckmann, C. F. (2015). ICA-AROMA: A robust ICA-based strategy for removing motion artifacts from fMRI data. Neurolmage, 112, 267-277. https://doi.org/10.1016/j.neuroimage.2015.02.064

Raichle, M. E. (2015). The Brain's Default Mode Network. Annual Review of Neuroscience, 38(1), 433-447. https://doi.org/10.1146/annurev-neuro071013-014030

Raichle, M. E., MacLeod, A. M., Snyder, A. Z., Powers, W. J., Gusnard, D. A., \& Shulman, G. L. (2001). A default mode of brain function. Proceedings of the National Academy of Sciences of the United States of America, 98(2), 676682. https://doi.org/10.1073/pnas.98.2.676

Ramasubbu, R., Lang, S., \& Kiss, Z. H. T. (2018). Dosing of electrical parameters in deep brain stimulation (DBS) for intractable depression: a review of clinical studies. Frontiers in Psychiatry, 9, 302.

Rapps, N., van Oudenhove, L., Enck, P., \& Aziz, Q. (2008). Brain imaging of visceral functions in healthy volunteers and IBS patients. Journal of Psychosomatic Research, 64(6), 599-604. https://doi.org/10.1016/j.jpsychores.2008.02.018

Rashid, B., Damaraju, E., Pearlson, G. D., \& Calhoun, V. D. (2014). Dynamic connectivity states estimated from resting fMRI Identify differences among Schizophrenia, bipolar disorder, and healthy control subjects. Frontiers in Human Neuroscience, 8(November), 897. https://doi.org/10.3389/fnhum.2014.00897

Ravizza, S. M., \& Carter, C. S. (2008). Shifting set about task switching: behavioral and neural evidence for distinct forms of cognitive flexibility. Neuropsychologia, 46(12), 2924-2935. https://doi.org/10.1016/j.neuropsychologia.2008.06.006

Reuter, M., Rosas, H. D., \& Fischl, B. (2010). Highly accurate inverse consistent registration: A robust approach. Neurolmage, 53(4), 1181-1196. https://doi.org/10.1016/j.neuroimage.2010.07.020

Revelle, W. (2010). psych: Procedures for personality and psychological research [Computer software manual]. In https://CRAN.Rproject.org/package =psych. Northwestern University. https://cran.rproject.org/package=psych Version $=1.9 .12$. 
Riess, H. (2017). The Science of Empathy. Journal of Patient Experience, 4(2), 74-77. https://doi.org/10.1177/2374373517699267

Roberts, R. D., Zeidner, M., \& Matthews, G. (2001). Does Emotional Intelligence Meet Traditional Standards for an Intelligence? Some New Data and Conclusions. In Emotion (Vol. 1, Issue 3, pp. 196-231). American Psychological Association. https://doi.org/10.1037/1528-3542.1.3.196

Rombouts, S. A. R. B., Barkhof, F., Hoogenraad, F. G. C., Sprenger, M., \& Scheltens, P. (1998). Within-subject reproducibility of visual activation patterns with functional magnetic resonance imaging using multislice echo planar imaging. Magnetic Resonance Imaging, 16(2), 105-113. https://doi.org/10.1016/S0730-725X(97)00253-1

Rossi, F., Giorgio, A., Battaglini, M., Stromillo, M. L., Portaccio, E., Goretti, B., Federico, A., Hakiki, B., Amato, M. P., \& De Stefano, N. (2012). Relevance of brain lesion location to cognition in relapsing multiple sclerosis. PloS One, 7(11), e44826-e44826. https://doi.org/10.1371/journal.pone.0044826

Rubia, K., Russell, T., Overmeyer, S., Brammer, M. J., Bullmore, E. T., Sharma, T., Simmons, A., Williams, S. C. R., Giampietro, V., Andrew, C. M., \& Taylor, E. (2001). Mapping motor inhibition: Conjunctive brain activations across different versions of go/no-go and stop tasks. Neurolmage, 13(2), 250-261. https://doi.org/10.1006/nimg.2000.0685

Rucker, D. D., Preacher, K. J., Tormala, Z. L., \& Petty, R. E. (2011). Mediation Analysis in Social Psychology: Current Practices and New Recommendations. Social and Personality Psychology Compass, 5(6), 359371. https://doi.org/10.1111/j.1751-9004.2011.00355.x

Rusticus, S. A., \& Lovato, C. Y. (2014). Impact of sample size and variability on the power and type I error rates of equivalence tests: A simulation study. Practical Assessment, Research and Evaluation, 19(11), 1-10.

Sabbagh, M. A., Xu, F., Carlson, S. M., Moses, L. J., \& Lee, K. (2006). The development of executive functioning and theory of mind: A comparison of Chinese and U.S. preschoolers. Psychological Science, 17(1), 74-81. https://doi.org/10.1111/j.1467-9280.2005.01667.x

Samson, A. C., Huber, O., \& Gross, J. J. (2012). Emotion regulation in Asperger's syndrome and high-functioning autism. Emotion, 12(4), 659-665. https://doi.org/10.1037/a0027975

Santiago, C., Herrmann, N., Swardfager, W., Saleem, M., Oh, P. I., Black, S. E., \& Lanctôt, K. L. (2015). White Matter Microstructural Integrity Is Associated with Executive Function and Processing Speed in Older Adults with Coronary Artery Disease. The American Journal of Geriatric Psychiatry, 23(7), 754-763. https://doi.org/10.1016/j.jagp.2014.09.008

Saxe, R., \& Powell, L. J. (2006). It's the thought that counts: Specific brain regions for one component of theory of mind. Psychological Science, 17(8), 692-699. https://doi.org/10.1111/j.1467-9280.2006.01768.x

Scarapicchia, V., Mazerolle, E. L., Fisk, J. D., Ritchie, L. J., \& Gawryluk, J. R. (2018). Resting state BOLD variability in Alzheimer's disease: A marker of 
cognitive decline or cerebrovascular status? In Frontiers in Aging

Neuroscience (Vol. 10, Issue FEB, p. 39).

https://doi.org/10.3389/fnagi.2018.00039

Schienle, A., \& Scharmüller, W. (2013). Cerebellar activity and connectivity during the experience of disgust and happiness. Neuroscience, 246, 375381. https://doi.org/10.1016/j.neuroscience.2013.04.048

Schmahmann, J. D. (2001). The cerebrocerebellar system: Anatomic substrates of the cerebellar contribution to cognition and emotion. International Review of Psychiatry, 13(4), 247-260. https://doi.org/10.1080/09540260120082092

Schmahmann, Jeremy D., \& Pandya, D. N. (1995). Prefrontal cortex projections to the basilar pons in rhesus monkey: implications for the cerebellar contribution to higher function. Neuroscience Letters, 199(3), 175-178. https://doi.org/10.1016/0304-3940(95)12056-A

Schnack, H. G., van Haren, N. E. M., Brouwer, R. M., Evans, A., Durston, S., Boomsma, D. I., Kahn, R. S., \& Hulshoff Pol, H. E. (2014). Changes in Thickness and Surface Area of the Human Cortex and Their Relationship with Intelligence. Cerebral Cortex, 25(6), 1608-1617. https://doi.org/10.1093/cercor/bht357

Schreiter, S., Pijnenborg, G. H. M., \& Aan Het Rot, M. (2013). Empathy in adults with clinical or subclinical depressive symptoms. Journal of Affective Disorders, 150(1), 1-16. https://doi.org/10.1016/j.jad.2013.03.009

Schuett, A. M. (2016). Attention Directs Emotion: Directed Attention Drives Emotional Intensity and Distinctiveness of Facial Perception. http://scholar.colorado.edu/honr_theses/1072/\%5Cnhttp://scholar.colorado.e $\mathrm{du} /$ cgi/viewcontent.cgi?article=2380\&context=honr_theses

Schulte-Rüther, M., Markowitsch, H. J., Shah, N. J., Fink, G. R., \& Piefke, M. (2008). Gender differences in brain networks supporting empathy. Neurolmage, 42(1), 393-403. https://doi.org/10.1016/j.neuroimage.2008.04.180

Schultz, W., Tremblay, L., \& Hollerman, J. R. (2000). Reward processing in primate orbitofrontal cortex and basal ganglia. Cerebral Cortex, 10(3), 272283. https://doi.org/10.1093/cercor/10.3.272

Schurz, M., Radua, J., Aichhorn, M., Richlan, F., \& Perner, J. (2014). Fractionating theory of mind: A meta-analysis of functional brain imaging studies. Neuroscience and Biobehavioral Reviews, 42, 9-34. https://doi.org/10.1016/j.neubiorev.2014.01.009

Sedeño, L., Couto, B., Melloni, M., Canales-Johnson, A., Yoris, A., Baez, S., Esteves, S., Velásquez, M., Barttfeld, P., Sigman, M., Kichic, R., Chialvo, D., Manes, F., Bekinschtein, T. A., \& Ibanez, A. (2014). How do you feel when you can't feel your body? Interoception, functional connectivity and emotional processing in depersonalization-derealization disorder. PLOS ONE, 9(6). https://doi.org/10.1371/journal.pone.0098769

Seeley, W. W., Menon, V., Schatzberg, A. F., Keller, J., Glover, G. H., Kenna, H., Reiss, A. L., \& Greicius, M. D. (2007). Dissociable intrinsic connectivity 
networks for salience processing and executive control. Journal of Neuroscience, 27(9), 2349-2356. https://doi.org/10.1523/JNEUROSCI.558706.2007

Segerstrom, S. C., Tsao, J. C. I., Alden, L. E., \& Craske, M. G. (2000). No Title. Cognitive Therapy and Research, 24(6), 671-688. https://doi.org/10.1023/a:1005587311498

Ségonne, F., Dale, A. M., Busa, E., Glessner, M., Salat, D., Hahn, H. K., \& Fischl, B. (2004). A hybrid approach to the skull stripping problem in MRI. Neurolmage, 22(3), 1060-1075. https://doi.org/10.1016/j.neuroimage.2004.03.032

Segonne, F., Pacheco, J., \& Fischl, B. (2007). Geometrically Accurate TopologyCorrection of Cortical Surfaces Using Nonseparating Loops. IEEE Transactions on Medical Imaging, 26(4), 518-529. https://doi.org/10.1109/TMl.2006.887364

Seth, A. K. (2013). Interoceptive inference, emotion, and the embodied self. Trends in Cognitive Sciences, 17(11), 565-573. https://doi.org/10.1016/j.tics.2013.09.007

Shamay-tsoory, S. (2015). The neuropsychology of empathy : evidence from lesion studies Neuropsychologie de I' apathie : apports des études lésionnelles. Rev Neuropsychol, 7(4), 237-243. https://doi.org/10.1684/nrp.2015.0356

Shamay-Tsoory, S G, Tomer, R., Berger, B. D., \& Aharon-Peretz, J. (2003). Characterization of Empathy Deficits following Prefrontal Brain Damage: The Role of the Right Ventromedial Prefrontal Cortex. Journal of Cognitive Neuroscience, 15(3), 324-337. https://doi.org/10.1162/089892903321593063

Shamay-Tsoory, Simone G., Aharon-Peretz, J., \& Perry, D. (2009). Two systems for empathy: A double dissociation between emotional and cognitive empathy in inferior frontal gyrus versus ventromedial prefrontal lesions. Brain, 132(3), 617-627. https://doi.org/10.1093/brain/awn279

Shamay-Tsoory, Simone G., Harari, H., Aharon-Peretz, J., \& Levkovitz, Y. (2010). The role of the orbitofrontal cortex in affective theory of mind deficits in criminal offenders with psychopathic tendencies. Cortex, 46(5), 668-677. https://doi.org/10.1016/j.cortex.2009.04.008

Shamay-Tsoory, Simone G., Shur, S., Harari, H., \& Levkovitz, Y. (2007). Neurocognitive Basis of Impaired Empathy in Schizophrenia. Neuropsychology, 21(4), 431-438. https://doi.org/10.1037/08944105.21.4.431

Shin, L. M., \& Liberzon, I. (2009). The Neurocircuitry of Fear, Stress, and Anxiety Disorders. Neuropsychopharmacology, 35(1), 169-191. https://doi.org/10.1038/npp.2009.83

Shu, J., Hassell, S., Weber, J., Ochsner, K. N., \& Mobbs, D. (2017). The role of empathy in experiencing vicarious anxiety. Journal of Experimental Psychology: General, 146(8), 1164-1188. 
https://doi.org/10.1037/xge0000335

Siegle, G. J., Steinhauer, S. R., Thase, M. E., Stenger, V. A., \& Carter, C. S. (2002). Can't shake that feeling: event-related fMRI assessment of sustained amygdala activity in response to emotional information in depressed individuals. Biological Psychiatry, 51(9), 693-707. https://doi.org/10.1016/s0006-3223(02)01314-8

Silton, N. R., \& Fogel, J. (2010). Religiosity, empathy, and psychopathology among young adult children of rabbis. Archive for the Psychology of Religion, 32(3), 277-291. https://doi.org/10.1163/157361210X532040

Simmonds, D. J., Pekar, J. J., \& Mostofsky, S. H. (2008). Meta-analysis of Go/No-go tasks demonstrating that $\mathrm{fMRI}$ activation associated with response inhibition is task-dependent. Neuropsychologia, 46(1), 224-232. https://doi.org/10.1016/j.neuropsychologia.2007.07.015

Sims, T. B., Neufeld, J., Johnstone, T., \& Chakrabarti, B. (2014). Autistic traits modulate frontostriatal connectivity during processing of rewarding faces. Social Cognitive and Affective Neuroscience, 9(12), 2010-2016. https://doi.org/10.1093/scan/nsu010

Singer, T., Critchley, H. D., \& Preuschoff, K. (2009). A common role of insula in feelings, empathy and uncertainty. Trends in Cognitive Sciences, 13(8), 334-340. https://doi.org/10.1016/j.tics.2009.05.001

Singer, T., Seymour, B., O'Doherty, J., Kaube, H., Dolan, R. J., \& Frith, C. D. (2004). Empathy for Pain Involves the Affective but not Sensory Components of Pain. Science, 303(5661), 1157-1162. https://doi.org/10.1126/science. 1093535

Sled, J. G., Zijdenbos, A. P., \& Evans, A. C. (1998). A nonparametric method for automatic correction of intensity nonuniformity in MRI data. IEEE Transactions on Medical Imaging, 17(1), 87-97. https://doi.org/10.1109/42.668698

Smith, S. M., \& Nichols, T. E. (2009). Threshold-free cluster enhancement: Addressing problems of smoothing, threshold dependence and localisation in cluster inference. Neurolmage, 44(1), 83-98. https://doi.org/10.1016/j.neuroimage.2008.03.061

Smolker, H. R., Depue, B. E., Reineberg, A. E., Orr, J. M., \& Banich, M. T. (2015). Individual differences in regional prefrontal gray matter morphometry and fractional anisotropy are associated with different constructs of executive function. Brain Structure \& Function, 220(3), 1291-1306. https://doi.org/10.1007/s00429-014-0723-y

Solso, S., Xu, R., Proudfoot, J., Hagler Jr, D. J., Campbell, K., Venkatraman, V., Carter Barnes, C., Ahrens-Barbeau, C., Pierce, K., Dale, A., Eyler, L., \& Courchesne, E. (2016). Diffusion Tensor Imaging Provides Evidence of Possible Axonal Overconnectivity in Frontal Lobes in Autism Spectrum Disorder Toddlers. Biological Psychiatry, 79(8), 676-684. https://doi.org/10.1016/j.biopsych.2015.06.029

Sommer, T., Richter, K., Singer, F., Derntl, B., Rune, G. M., Diekhof, E., \& Bayer, 
J. (2018). Effects of the experimental administration of oral estrogen on prefrontal functions in healthy young women. Psychopharmacology, 235(12), 3465-3477. https://doi.org/10.1007/s00213-018-5061-y

Sonnby-Borgström, M. (2002). Automatic mimicry reactions as related to differences in emotional empathy. Scandinavian Journal of Psychology, 43(5), 433-443. https://doi.org/10.1111/1467-9450.00312

Sowell, E R, Thompson, P. M., Tessner, K. D., \& Toga, A. W. (2001). Mapping continued brain growth and gray matter density reduction in dorsal frontal cortex: Inverse relationships during postadolescent brain maturation. The Journal of Neuroscience: The Official Journal of the Society for Neuroscience, 21(22), 8819-8829. https://doi.org/10.1523/JNEUROSCI.2122-08819.2001

Sowell, Elizabeth R, Thompson, P. M., Holmes, C. J., Jernigan, T. L., \& Toga, A. W. (1999). In vivo evidence for post-adolescent brain maturation in frontal and striatal regions. Nature Neuroscience, 2(10), 859-861. https://doi.org/10.1038/13154

Spielberger, C. D., Edwards, C. D., Montouri, J., \& Lushene, R. (1973). StateTrait Anxiety Inventory for Children. In PsycTESTS Dataset. American Psychological Association (APA). https://doi.org/10.1037/t06497-000

Spreng, R. N., Mar, R. A., \& Kim, A. S. N. (2009). The common neural basis of autobiographical memory, prospection, navigation, theory of mind, and the default mode: A quantitative meta-analysis. Journal of Cognitive Neuroscience, 21(3), 489-510. https://doi.org/10.1162/jocn.2008.21029

Spreng, R. N., McKinnon, M. C., Mar, R. A., \& Levine, B. (2009). The Toronto Empathy Questionnaire: Scale Development and Initial Validation of a Factor-Analytic Solution to Multiple Empathy Measures. Journal of Personality Assessment, 91(1), 62-71. https://doi.org/10.1080/00223890802484381

Stein, M. B., Simmons, A. N., Feinstein, J. S., \& Paulus, M. P. (2007). Increased amygdala and insula activation during emotion processing in anxiety-prone subjects. American Journal of Psychiatry, 164(2), 318-327.

Stein, R. B., Gossen, E. R., \& Jones, K. E. (2005). Neuronal variability: Noise or part of the signal? Nature Reviews Neuroscience, 6(5), 389-397. https://doi.org/10.1038/nrn1668

Steinbeis, N. (2016). The role of self-other distinction in understanding others' mental and emotional states: Neurocognitive mechanisms in children and adults. Philosophical Transactions of the Royal Society B: Biological Sciences, 371(1686), 20150074. https://doi.org/10.1098/rstb.2015.0074

Steinberg, L. (2005). Cognitive and affective development in adolescence. Trends in Cognitive Sciences, 9(2), 69-74. https://doi.org/10.1016/j.tics.2004.12.005

Sternberg. (2000). Emotional Intelligence Checklist Emotional Intelligence. Business, 78(2), 1-4.

Stevens, F. L., Hurley, R. A., \& Taber, K. H. (2011). Anterior cingulate cortex: 
Unique role in cognition and emotion. Journal of Neuropsychiatry and Clinical Neurosciences, 23(2), 121-125.

https://doi.org/10.1176/jnp.23.2.jnp121

Stoet, G. (2010). Sex differences in the processing of flankers. Quarterly Journal of Experimental Psychology, 63(4), 633-638. https://doi.org/10.1080/17470210903464253

Strack, F., Martin, L. L., \& Stepper, S. (1988). Inhibiting and Facilitating Conditions of the Human Smile: A Nonobtrusive Test of the Facial Feedback Hypothesis. Journal of Personality and Social Psychology, 54(5), 768-777. https://doi.org/10.1037/0022-3514.54.5.768

Strayer, J. (1993). Children's Concordant Emotions and Cognitions in Response to Observed Emotions. Child Development, 64(1), 188-201. https://doi.org/10.1111/j.1467-8624.1993.tb02903.x

Stroop, J. R. (1935). Studies of interference in serial verbal reactions. Journal of Experimental Psychology, 18(6), 643-662. https://doi.org/10.1037/h0054651

Stuss, D. T., \& Levine, B. (2002). Adult clinical neuropsychology: Lessons from studies of the frontal lobes. Annual Review of Psychology, 53(1), 401-433. https://doi.org/10.1146/annurev.psych.53.100901.135220

Tajadura-Jiménez, A., \& Tsakiris, M. (2014). Balancing the "inner" and the "outer" self: Interoceptive sensitivity modulates self-other boundaries. In Journal of Experimental Psychology: General (Vol. 143, Issue 2, pp. 736-744). American Psychological Association. https://doi.org/10.1037/a0033171

Takeuchi, H., Taki, Y., Nouchi, R., Hashizume, H., Sekiguchi, A., Kotozaki, Y., Nakagawa, S., Miyauchi, C. M., Sassa, Y., \& Kawashima, R. (2014). Effects of multitasking-training on gray matter structure and resting state neural mechanisms. Human Brain Mapping, 35(8), 3646-3660. https://doi.org/10.1002/hbm.22427

Tamm, L., Barnea-Goraly, N., \& Reiss, A. L. (2012). Diffusion tensor imaging reveals white matter abnormalities in Attention-Deficit/Hyperactivity Disorder. Psychiatry Research, 202(2), 150-154. https://doi.org/10.1016/j.pscychresns.2012.04.001

Tamres, L. K., Janicki, D., \& Helgeson, V. S. (2002). Sex Differences in Coping Behavior: A Meta-Analytic Review and an Examination of Relative Coping. Personality and Social Psychology Review, 6(1), 2-30. https://doi.org/10.1207/S15327957PSPR0601_1

Tamura, I., Kitagawa, M., Otsuki, M., Kikuchi, S., Tashiro, K., \& Dubois, B. (2007). Pure Topographical Disorientation Following a Right Forceps Major of the Splenium Lesion: A Case Study. Neurocase, 13(3), 178-184. https://doi.org/10.1080/13554790701448812

Tarasuik, J. C., Ciorciari, J., \& Stough, C. (2009). Understanding the Neurobiology of Emotional Intelligence: A Review (pp. 307-320). https://doi.org/10.1007/978-0-387-88370-0_16

Tavor, I., Parker Jones, O., Mars, R. B., Smith, S. M., Behrens, T. E., \& Jbabdi, S. (2016). Task-free MRI predicts individual differences in brain activity 
during task performance. Science, 352(6282), 216-220.

https://doi.org/10.1126/science.aad8127

Taylor, G. J. (2001). Low emotional intelligence and mental illness. In Emotional intelligence in everyday life: A scientific inquiry. (pp. 67-81). Psychology Press.

Taylor, K. S., Seminowicz, D. A., \& Davis, K. D. (2009). Two systems of resting state connectivity between the insula and cingulate cortex. Human Brain Mapping, 30(9), 2731-2745. https://doi.org/10.1002/hbm.20705

Taylor, R., Théberge, J., Williamson, P. C., Densmore, M., \& Neufeld, R. W. J. (2016). ACC Neuro-over-Connectivity Is Associated with Mathematically Modeled Additional Encoding Operations of Schizophrenia Stroop-Task Performance. Frontiers in Psychology, 7, 1295.

https://doi.org/10.3389/fpsyg.2016.01295

Team. (2020). RStudio. PBC. http://www.rstudio.com

Terasawa, Y., Fukushima, H., \& Umeda, S. (2013). How does interoceptive awareness interact with the subjective experience of emotion? An fMRI Study. Human Brain Mapping, 34(3), 598-612. https://doi.org/10.1002/hbm.21458

Thayer, R. E., Newman, J. R., \& McClain, T. M. (1994). Self-Regulation of Mood: Strategies for Changing a Bad Mood, Raising Energy, and Reducing Tension. In Journal of Personality and Social Psychology (Vol. 67, Issue 5). https://doi.org/10.1037/0022-3514.67.5.910

Thoma, P., Zalewski, I., von Reventlow, H. G., Norra, C., Juckel, G., \& Daum, I. (2011). Cognitive and affective empathy in depression linked to executive control. Psychiatry Research, 189(3), 373-378. https://doi.org/10.1016/j.psychres.2011.07.030

Thompson, W. F., Schellenberg, E. G., \& Husain, G. (2004). Decoding Speech Prosody: Do Music Lessons Help? Emotion, 4(1), 46-64. https://doi.org/10.1037/1528-3542.4.1.46

Thomsen, D. K., Mehlsen, M. Y., Viidik, A., Sommerlund, B., \& Zachariae, R. (2005). Age and gender differences in negative affect - Is there a role for emotion regulation? Personality and Individual Differences, 38(8), 19351946. https://doi.org/10.1016/j.paid.2004.12.001

Timmers, M., Fischer, A. H., \& Manstead, A. S. R. (2003). Ability versus vulnerability: Beliefs about men's and women's emotional behaviour. In Cognition and Emotion (Vol. 17, Issue 1, pp. 41-63). https://doi.org/10.1080/02699930302277

Todorov, A., \& Engell, A. D. (2008). The role of the amygdala in implicit evaluation of emotionally neutral faces. Social Cognitive and Affective Neuroscience, 3(4), 303-312. https://doi.org/10.1093/scan/nsn033

Tognoli, E., \& Kelso, J. A. S. (2014). The Metastable Brain. Neuron, 81(1), 3548. https://doi.org/10.1016/j.neuron.2013.12.022

Tollefson, J. (2019). Humans are driving one million species to extinction. In Nature (Vol. 569, Issue 7755, p. 171). Nature Publishing Group. 
https://doi.org/10.1038/d41586-019-01448-4

Tone, E. B., \& Tully, E. C. (2014). Empathy as a risky strength: A multilevel examination of empathy and risk for internalizing disorders. Development and Psychopathology, 26(4 0 2), 1547-1565. https://doi.org/10.1017/S0954579414001199

Tops, M., Boksem, M. A. S., Luu, P., \& Tucker, D. M. (2011). Brain substrates of behavioral programs associated with self-regulation. Frontiers in Psychology, 2(AUG), 152. https://doi.org/10.3389/fpsyg.2010.00152

Tops, M., \& De Jong, R. (2006). Posing for success: Clenching a fist facilitates approach. Psychonomic Bulletin and Review, 13(2), 229-234. https://doi.org/10.3758/BF03193835

Tremblay, L., \& Schultz, W. (1999). Relative reward preference in primate orbitofrontal cortex. Nature, 398(6729), 704-708. https://doi.org/10.1038/19525

Treynor, W. (2003). No Title. Cognitive Therapy and Research, 27(3), 247-259. https://doi.org/10.1023/a:1023910315561

Trilla, Panasiti, M. S., \& Chakrabarti, B. (2015). The plasticity of the mirror system: How reward learning modulates cortical motor simulation of others. Neuropsychologia, 70, 255-262. https://doi.org/https://doi.org/10.1016/j.neuropsychologia.2015.02.033

Tsakiris, M., Tajadura-Jiménez, A., \& Costantini, M. (2011). Just a heartbeat away from one's body:Interoceptive sensitivity predicts malleability of bodyrepresentations. Proceedings of the Royal Society B: Biological Sciences, 278(1717), 2470-2476. https://doi.org/10.1098/rspb.2010.2547

Urry, H. L., van Reekum, C. M., Johnstone, T., \& Davidson, R. J. (2009). Individual differences in some (but not all) medial prefrontal regions reflect cognitive demand while regulating unpleasant emotion. Neurolmage, 47(3), 852-863. https://doi.org/10.1016/j.neuroimage.2009.05.069

Vakorin, V. A., Lippe, S., \& Mclntosh, A. R. (2011). Variability of brain signals processed locally transforms into higher connectivity with brain development. Journal of Neuroscience, 31(17), 6405-6413. https://doi.org/10.1523/JNEUROSCI.3153-10.2011

Vakorin, V. A., Mišić, B., Krakovska, O., \& Mcintosh, A. R. (2011). Empirical and theoretical aspects of generation and transfer of information in a neuromagnetic source network. Frontiers in Systems Neuroscience, 5(NOVEMBER 2011), 96. https://doi.org/10.3389/fnsys.2011.00096

van den Heuvel, M. P., \& Hulshoff Pol, H. E. (2010). Exploring the brain network: A review on resting-state fMRI functional connectivity. In European Neuropsychopharmacology (Vol. 20, Issue 8, pp. 519-534). https://doi.org/10.1016/j.euroneuro.2010.03.008

van der Heiden, L., Scherpiet, S., Konicar, L., Birbaumer, N., \& Veit, R. (2013). Inter-individual differences in successful perspective taking during pain perception mediates emotional responsiveness in self and others: An fMRI study. Neurolmage, 65, 387-394. 
https://doi.org/10.1016/j.neuroimage.2012.10.003

Vanderhasselt, M.-A., Kühn, S., \& De Raedt, R. (2011). Healthy brooders employ more attentional resources when disengaging from the negative: an eventrelated fMRI study. Cognitive, Affective, \& Behavioral Neuroscience, 11(2), 207-216. https://doi.org/10.3758/s13415-011-0022-5

Vanderwal, T., Kelly, C., Eilbott, J., Mayes, L. C., \& Castellanos, F. X. (2015). Inscapes: A movie paradigm to improve compliance in functional magnetic resonance imaging. Neurolmage, 122, 222-232. https://doi.org/10.1016/j.neuroimage.2015.07.069

Viviani, R. (2013). Emotion regulation, attention to emotion, and the ventral attentional network. In Frontiers in Human Neuroscience (Vol. 7, Issue NOV, p. 746). https://doi.org/10.3389/fnhum.2013.00746

Vladimir, K., Elena, V., Ekaterina, K., \& Pavel, E. (2019). A review of neurophysiological and genetic correlates of emotional intelligence. International Journal of Cognitive Research in Science, Engineering and Education, 7(1), 137-142. https://doi.org/10.5937/ijcrsee1901137K

Vogeley, K., Bussfeld, P., Newen, A., Herrmann, S., Happé, F., Falkai, P., Maier, W., Shah, N. J., Fink, G. R., \& Zilles, K. (2001). Mind reading: Neural mechanisms of theory of mind and self-perspective. Neurolmage, 14(1 I), 170-181. https://doi.org/10.1006/nimg.2001.0789

von dem Hagen, E. A. H., Stoyanova, R. S., Baron-Cohen, S., \& Calder, A. J. (2012). Reduced functional connectivity within and between 'social' resting state networks in autism spectrum conditions. Social Cognitive and Affective Neuroscience, 8(6), 694-701. https://doi.org/10.1093/scan/nss053

Votinov, M., Wagels, L., Hoffstaedter, F., Kellermann, T., Goerlich, K. S., Eickhoff, S. B., \& Habel, U. (2020). Effects of exogenous testosterone application on network connectivity within emotion regulation systems. Scientific Reports, 10(1), 2352. https://doi.org/10.1038/s41598-020-59329-0 WABER, D. P., DE MOOR, C., FORBES, P. W., ALMLI, C. R., BOTTERON, K. N., LEONARD, G., MILOVAN, D., PAUS, T., \& RUMSEY, J. (2007). The NIH MRI study of normal brain development: Performance of a population based sample of healthy children aged 6 to 18 years on a neuropsychological battery. Journal of the International Neuropsychological Society, 13(05). https://doi.org/10.1017/s1355617707070841

Waber, D. P., Forbes, P. W., Almli, C. R., Blood, E. A., \& Group, B. D. C. (2012). Four-year longitudinal performance of a population-based sample of healthy children on a neuropsychological battery: the NIH MRI study of normal brain development. Journal of the International Neuropsychological Society: JINS, 18(2), 179-190. https://doi.org/10.1017/S1355617711001536

Watkins, E. R., \& Moulds, M. L. (2009). Thought Control Strategies, Thought Suppression, and Rumination in Depression. International Journal of Cognitive Therapy, 2(3), 235-251. https://doi.org/10.1521/ijct.2009.2.3.235

Webb, T. L., Miles, E., \& Sheeran, P. (2012). Dealing with feeling: A metaanalysis of the effectiveness of strategies derived from the process model of 
emotion regulation. In Psychological Bulletin (Vol. 138, Issue 4, pp. 775808). American Psychological Association. https://doi.org/10.1037/a0027600

Weilbächer, R. A., \& Gluth, S. (2017). The interplay of hippocampus and ventromedial prefrontal cortex in memory-based decision making. In Brain Sciences (Vol. 7, Issue 1). MDPI AG. https://doi.org/10.3390/brainsci7010004

Weissman, D. H., Gopalakrishnan, A., Hazlett, C. J., \& Woldorff, M. G. (2005). Dorsal anterior cingulate cortex resolves conflict from distracting stimuli by boosting attention toward relevant events. Cerebral Cortex, 15(2), 229-237. https://doi.org/10.1093/cercor/bhh125

Werner, N. S., Duschek, S., Mattern, M., \& Schandry, R. (2009). Interoceptive sensitivity modulates anxiety during public speaking. Journal of Psychophysiology, 23(2), 85-94. https://doi.org/10.1027/0269-8803.23.2.85

Whitfield-Gabrieli, S., \& Nieto-Castanon, A. (2012). Conn: A Functional Connectivity Toolbox for Correlated and Anticorrelated Brain Networks. Brain Connectivity, 2(3), 125-141. https://doi.org/10.1089/brain.2012.0073

Whittle, S., Yücel, M., Yap, M. B. H., \& Allen, N. B. (2011). Sex differences in the neural correlates of emotion: Evidence from neuroimaging. In Biological Psychology (Vol. 87, Issue 3, pp. 319-333). https://doi.org/10.1016/j.biopsycho.2011.05.003

Wicker, B., Keysers, C., Plailly, J., Royet, J. P., Gallese, V., \& Rizzolatti, G. (2003). Both of us disgusted in My insula: The common neural basis of seeing and feeling disgust. Neuron, 40(3), 655-664. https://doi.org/10.1016/S0896-6273(03)00679-2

Wide, J. K., Hanratty, K., Ting, J., \& Galea, L. A. M. (2004). High level estradiol impairs and low level estradiol facilitates non-spatial working memory. Behavioural Brain Research, 155(1), 45-53. https://doi.org/10.1016/j.bbr.2004.04.001

Wiens, S. (2005). Interoception in emotional experience. In Current Opinion in Neurology (Vol. 18, Issue 4, pp. 442-447). Lippincott Williams and Wilkins. https://doi.org/10.1097/01.wco.0000168079.92106.99

Wild, B., Erb, M., \& Bartels, M. (2001). Are emotions contagious? Evoked emotions while viewing emotionally expressive faces: Quality, quantity, time course and gender differences. Psychiatry Research, 102(2), 109-124. https://doi.org/10.1016/S0165-1781(01)00225-6

Witte, A. V., Savli, M., Holik, A., Kasper, S., \& Lanzenberger, R. (2010). Regional sex differences in grey matter volume are associated with sex hormones in the young adult human brain. Neurolmage, 49(2), 1205-1212. https://doi.org/10.1016/j.neuroimage.2009.09.046

Wozniak, J. R., Krach, L., Ward, E., Mueller, B. A., Muetzel, R., Schnoebelen, S., Kiragu, A., \& Lim, K. O. (2007). Neurocognitive and neuroimaging correlates of pediatric traumatic brain injury: a diffusion tensor imaging (DTI) study. Archives of Clinical Neuropsychology: The Official Journal of the National Academy of Neuropsychologists, 22(5), 555-568. 
https://doi.org/10.1016/j.acn.2007.03.004

Wu, Yan, Li, H., Zhou, Y., Yu, J., Zhang, Y., Song, M., Qin, W., Yu, C., \& Jiang, T. (2016). Sex-specific neural circuits of emotion regulation in the centromedial amygdala. Scientific Reports, 6. https://doi.org/10.1038/srep23112

Wu, Yan, Wang, J., Zhang, Y., Zheng, D., Zhang, J., Rong, M., Wu, H., Wang, Y., Zhou, K., \& Jiang, T. (2016). The neuroanatomical basis for posterior superior parietal lobule control lateralization of visuospatial attention. Frontiers in Neuroanatomy, 10(MAR), 32. https://doi.org/10.3389/fnana.2016.00032

Wu, Yupeng, Sun, D., Wang, Y., \& Wang, Y. (2016). Subcomponents and Connectivity of the Inferior Fronto-Occipital Fasciculus Revealed by Diffusion Spectrum Imaging Fiber Tracking. Frontiers in Neuroanatomy, 10, 88. https://doi.org/10.3389/fnana.2016.00088

Xiang, Y., Zhao, S., Wang, H., Wu, Q., Kong, F., \& Mo, L. (2017). Examining brain structures associated with dispositional envy and the mediation role of emotional intelligence. Scientific Reports, 7(1), 39947. https://doi.org/10.1038/srep39947

Yamagata, T., Nakayama, Y., Tanji, J., \& Hoshi, E. (2012). Distinct information representation and processing for goal-directed behavior in the dorsolateral and ventrolateral prefrontal cortex and the dorsal premotor cortex. The Journal of Neuroscience: The Official Journal of the Society for Neuroscience, 32(37), 12934-12949. https://doi.org/10.1523/JNEUROSCI.2398-12.2012

Yamasaki, H., LaBar, K. S., \& McCarthy, G. (2002). Dissociable prefrontal brain systems for attention and emotion. Proceedings of the National Academy of Sciences of the United States of America, 99(17), 11447-11451. https://doi.org/10.1073/pnas.182176499

Yan, Z., Hong, S., Liu, F., \& Su, Y. (2020). A meta-analysis of the relationship between empathy and executive function. PsyCh Journal, 9(1), 34-43. https://doi.org/10.1002/pchj.311

Yeo, Krienen, F. M., Sepulcre, J., Sabuncu, M. R., Lashkari, D., Hollinshead, M., Roffman, J. L., Smoller, J. W., Zöllei, L., Polimeni, J. R., Fisch, B., Liu, H., \& Buckner, R. L. (2011). The organization of the human cerebral cortex estimated by intrinsic functional connectivity. Journal of Neurophysiology, 106(3), 1125-1165. https://doi.org/10.1152/jn.00338.2011

Young, L., Dodell-Feder, D., \& Saxe, R. (2010). What gets the attention of the temporo-parietal junction? An fMRI investigation of attention and theory of mind. Neuropsychologia, 48(9), 2658-2664.

https://doi.org/10.1016/j.neuropsychologia.2010.05.012

Yuan, P., \& Raz, N. (2014). Prefrontal cortex and executive functions in healthy adults: a meta-analysis of structural neuroimaging studies. Neuroscience and Biobehavioral Reviews, 42, 180-192. https://doi.org/10.1016/j.neubiorev.2014.02.005 
Yurgelun-Todd, D. (2007). Emotional and cognitive changes during adolescence. Current Opinion in Neurobiology, 17(2), 251-257.

https://doi.org/10.1016/j.conb.2007.03.009

Zahn-Waxler, C., Cole, P. M., \& Barrett, K. C. (2010). Guilt and empathy: Sex differences and implications for the development of depression. The Development of Emotion Regulation and Dysregulation, 243-272. https://doi.org/10.1017/cbo9780511663963.012

Zaki. (2014). Empathy: A motivated account. Psychological Bulletin, 140(6), 1608-1647. https://doi.org/10.1037/a0037679

Zaki, J., Davis, J. I., \& Ochsner, K. N. (2012). Overlapping activity in anterior insula during interoception and emotional experience. Neurolmage, 62(1), 493-499. https://doi.org/10.1016/j.neuroimage.2012.05.012

Zaki, J., \& Ochsner, K. (2012). The neuroscience of empathy: Progress, pitfalls and promise. In Nature Neuroscience (Vol. 15, Issue 5, pp. 675-680). Nature Publishing Group. https://doi.org/10.1038/nn.3085

Zhang, L., Zuo, X. N., Ng, K. K., Chong, J. S. X., Shim, H. Y., Ong, M. Q. W., Loke, Y. M., Choo, B. L., Chong, E. J. Y., Wong, Z. X., Hilal, S., Venketasubramanian, N., Tan, B. Y., Chen, C. L. H., \& Zhou, J. H. (2020). Distinct BOLD variability changes in the default mode and salience networks in Alzheimer's disease spectrum and associations with cognitive decline. Scientific Reports, 10(1), 6457. https://doi.org/10.1038/s41598-020-63540-4

Zhao, X., Lynch, J. G., \& Chen, Q. (2010). Reconsidering Baron and Kenny: Myths and Truths about Mediation Analysis. Journal of Consumer Research, 37(2), 197-206. https://doi.org/10.1086/651257 


\section{CURRICULUM VITA}

NAME: Teodora Stoica

ADDRESS: Interdisciplinary Department

Houchens Building, Suite 105

University of Louisville

Louisville, Kentucky 40292

DOB: $\quad$ Pitesti, Romania - February 7, 1986

\section{EDUCATION}

\& TRAINING: $\quad$ B.A. Psychology

East Carolina University

2004-2007

M.S. Neuroscience

University of Hartford

2009-2010

Ph.D. Translational Neuroscience

University of Louisville

2015-2021

AWARDS: Dissertation Completion Award

2020

Graduate Intramural Research and Creative Activities Grant 2020

Dissertation Award

2019

Excellence in Service Award

2019

Next Generation Award

2018

Cloud Computing Scholarship

2017

Graduate Research Grant

2017 
Travel Scholarship for Brainhack Course

2017

Outstanding Trainee Award

2017

Travel Award

2013

Carol Ripley Scholarship for Outstanding Thesis

2007

PROFESSIONAL SOCIETES: Cognitive Neuroscience Society

Organization for Human Brain Mapping

Society for Neuroscience

American Psychological Association

Social and Affective Neuroscience Society

\section{PUBLICATIONS:}

1. Stoica, T., Depue, B.E. Resting State Relationship between Alexithymia and Emotion Regulation Strategy Mediated by Personality Traits in Large Sample: A Dynamic Connectivity Study (In Preparation)

2. Stoica, T., Depue, B.E.(2021) Interoceptive Awareness and Empathy Share Intrinsic Spatial and Temporal Connectivity. Frontiers in Human Neuroscience 3. Stoica, T., Knight L.K, Naaz, F., Patton, S.C., Depue, B.E. (2021) Functional Connectivity Gender Differences during Emotion Regulation. Neuropsychologia 4. Stoica T., Depue, B.E. Neuroimaging Insights into Common Behavioral Phenotypes of Autism Spectrum Disorders. Textbook of Clinical and Basic Neuroscience in Autism Spectrum Disorders (Book Chapter, In Press) 5. Stoica, T., Knight, L.K, Naaz, F., Ramic, M., Depue, B.E (2019) Structural Morphometry and Connectivity of the Lateral Prefrontal Cortex Relates to Executive Function and Estradiol level in healthy adolescents.B rain and Behavior 6. Knight, L.K, Stoica, T., Fogleman, N., Depue, B.E. (2019) Convergent Neural Correlates of Empathy and Anxiety during Socioemotional Processing.F rontiers in Human Neuroscience

7. Fogleman, N., Naaz, F., Knight, L., Stoica, T., Patton, S., \& Olson-Madden, J. et al. (2017). Reduced lateral prefrontal cortical volume is associated with performance on the modified Iowa Gambling Task: A surface based morphometric analysis of previously deployed veterans. Psychiatry Research: Neuroimaging 8. Knight, L., Naaz, F., Stoica, T., \& Depue, B. (2017). Lifetime PTSD and geriatric depression symptomatology relate to altered dorsomedial frontal and amygdala morphometry. Psychiatry Research: Neuroimaging 9. Evon, D., Golin, C., Stoica, T., Jones, R., Willis, S., Galanko, J., \& Fried, M. (2016). What's Important to the Patient? Informational Needs of Patients Making Decisions About Hepatitis C Treatment. The Patient - Patient-Centered Outcomes Research 
10. Radua, J., Stoica, T., Scheinost, D., Pittenger, C., \& Hampson, M. (2016).

Neural Correlates of Success and Failure Signals During Neurofeedback

Learning. Neuroscience

11. Scheinost, D., Stoica, T., Wasylink, S., Gruner, P., Saksa, J., Pittenger, C., \& Hampson, M.(2014). Resting state functional connectivity predicts neurofeedback response. Frontiers In Behavioral Neuroscience

12. Hampson, M., Stoica, T., Saksa, J., Scheinost, D., Qiu, M., \& Bhawnani, J. et al.(2012). Real-time fMRI Biofeedback Targeting the Orbitofrontal Cortex for Contamination Anxiety. Journal Of Visualized Experiments

13. Hampson M., Stoica T., Saksa J.R., Scheinost D., Qiu M., Bhawnani J., Pittenger, C., Papademetris X., Constable R.T. (2013). Biofeedback of real-time fMRI data from OFC to reduce contamination anxiety Translational Psychiatry

\section{NATIONAL MEETING PRESENTATIONS:}

(Poster) Society for Affective Neuroscience (SANS), Virtual, April (Participant) NeuroHackacademy, Virtual, July-August

(Participant) Organization for Computational Neurosciences, Virtual, July

(Participant) Organization for Human Brain Mapping, Virtual, July

(Participant) Reading Emotions Symposium, University of Reading, Virtual, June

*(Workshop) Brain Connectivity Workshop, Toronto, Canada, June

*(Workshop) Summer School in Social Neuroscience, Durham, NC June (Participant) Society for Affective Science, Virtual, May

(Poster) Cognitive Neuroscience Society, Virtual, May

*(Poster) Society and Affective Neuroscience Society, Santa Barbara, CA May

*Event canceled due to COVID-19 Pandemic

(Invited Outreach Keynote) Society for Neuroscience (SfN), Chicago, IL October

(Poster) Organization for Human Brain Mapping, Rome, Italy July

(Poster) Graduate Student Research Conference, U of Louisville, February

(Talk) Society for Neuroscience (SfN), San Diego, CA November

(Poster) Neuroscience Day, U of Louisville April

(Poster) Graduate Student Research Conference, U of Louisville March

(Poster) Cognitive Neuroscience Society, Boston MA March

(Poster) Organization for Human Brain Mapping, Vancouver, BC June 2017

(Poster) Cognitive Neuroscience Society, San Francisco, CA April 2017

(Talk/Poster) Neuroscience Day, University of Louisville March 2017

(Poster) Neuroscience Day, University of Louisville April 2016

(Talk/Poster) Cognitive Neuroscience Society, New York, NY April 2016

(Poster) Research!Louisville October 2015

(Poster) SBMT Baltimore MD May 2013

(Talk/Poster) National TBI Research Symposium, Bethesda MD April 2013

(Poster) ISMRM, Salt Lake City, UT April 2013 